\title{
BRAINCASE OF THE UPPER DEVONIAN SHARK CLADODOIDES WILDUNGENSIS (CHONDRICHTHYES, ELASMOBRANCHII), WITH OBSERVATIONS ON THE BRAINCASE IN EARLY CHONDRICHTHYANS
}

\author{
JOHN G. MAISEY \\ Division of Paleontology \\ American Museum of Natural History \\ (maisey@amnh.org)
}

BULLETIN OF THE AMERICAN MUSEUM OF NATURAL HISTORY

CENTRAL PARK WEST AT 79TH STREET, NEW YORK, NY 10024

Number 288, 103 pp., 38 figures

Issued March 2, 2005 


\section{CONTENTS}

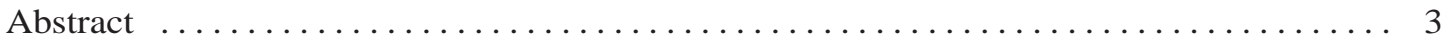

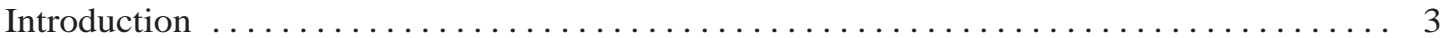

Materials and Methods $\ldots \ldots \ldots \ldots \ldots \ldots \ldots \ldots \ldots \ldots \ldots \ldots \ldots \ldots \ldots \ldots \ldots$

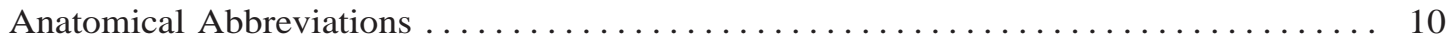

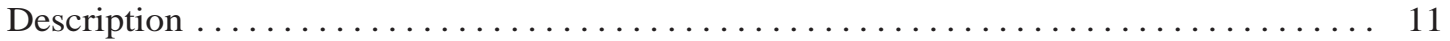

General Features, Taphonomy, and Forensic Observations $\ldots \ldots \ldots \ldots \ldots \ldots \ldots \ldots$

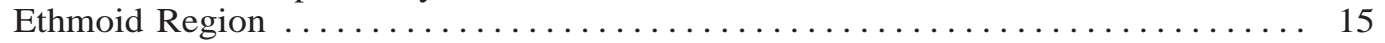

Extent of Polar Cartilage-Derived Region $\ldots \ldots \ldots \ldots \ldots \ldots \ldots \ldots \ldots \ldots \ldots \ldots \ldots \ldots \ldots \ldots$

Ophthalmic and Efferent Pseudobranchial Arteries . . . . . . . . . . . . . . . 19

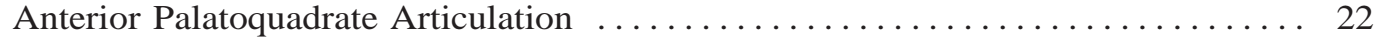

Bucco-Hypophyseal Fenestra and Internal Carotid Arteries . . . . . . . . . . . 22

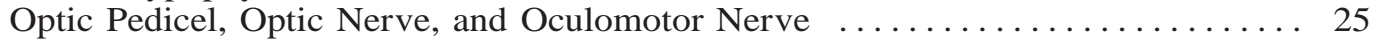

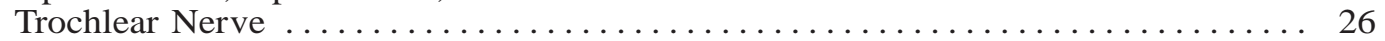

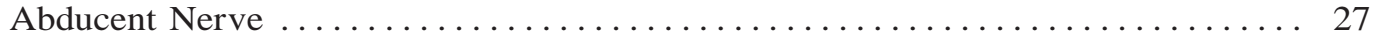

Profundal Nerve ... . . . . . . . . . . . . . . . . . . . . . . . . . . . 30

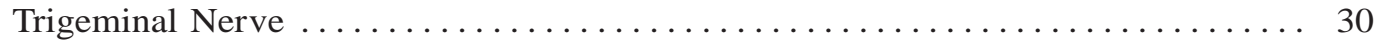

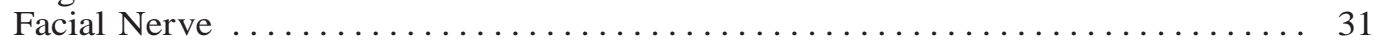

Octavolateral Nerves . . . . . . . . . . . . . . . . . . . . . . . . . . 34

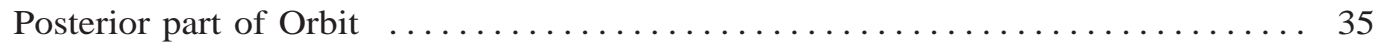

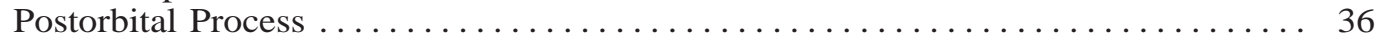

Dorsal Aortae ... . . . . . . . . . . . . . . . . . . . . . . . . . . . . . . . . . 39

Orbital Artery and Palatine Nerve ...................... 40

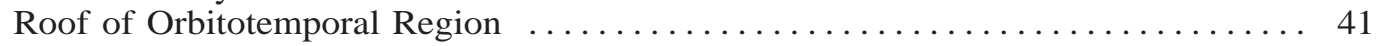

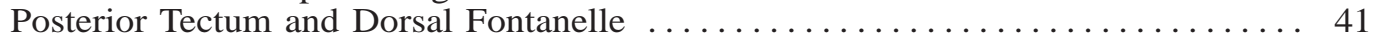

Dorsal Otic Ridges . . . . . . . . . . . . . . . . . . . . . . . . . . . . 42

Lateral Otic Process and Hyomandibular Fossa $\ldots \ldots \ldots \ldots \ldots \ldots \ldots \ldots \ldots$

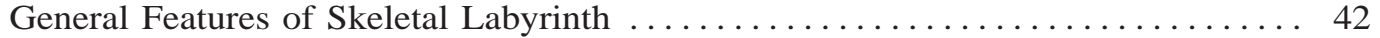

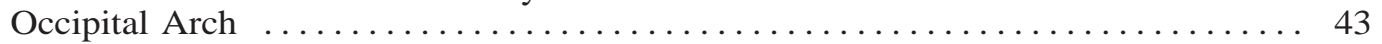

Cranial Endocast ................................. 52

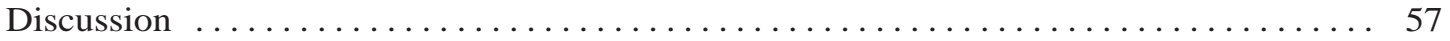

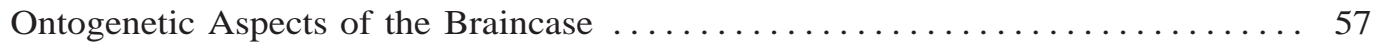

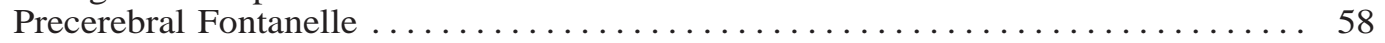

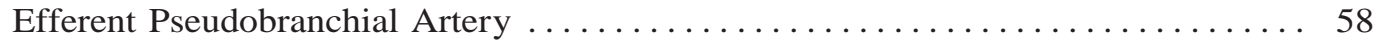

Anterior Palatoquadrate Articulation ... . . . . . . . . . . . . . . . . 59

Trabeculae, Polar Cartilage, and Suborbital Shelf . . . . . . . . . . . . . . . 64

Optic Pedicel and Position of Oculomotor Foramen $\ldots \ldots \ldots \ldots \ldots \ldots \ldots \ldots \ldots$

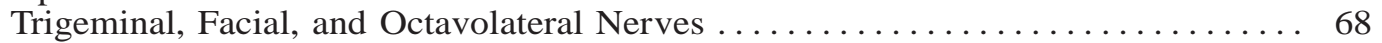

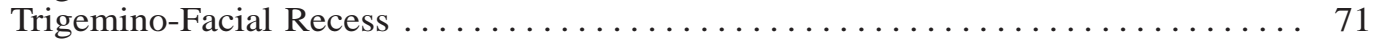

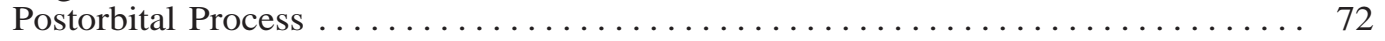

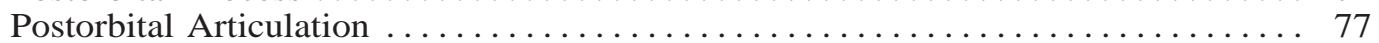

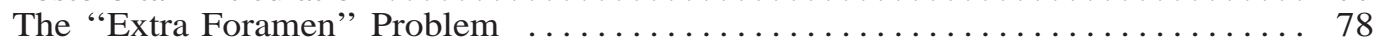

Remarks on the Dorsal Vein Sinus in Acanthodes $\ldots \ldots \ldots \ldots \ldots \ldots \ldots \ldots \ldots \ldots \ldots . . \ldots 1$

Otico-Occipital Proportions $\ldots \ldots \ldots \ldots \ldots \ldots \ldots \ldots \ldots \ldots \ldots \ldots \ldots \ldots \ldots \ldots \ldots$

Remarks on the Inner Ear in Chondrichthyans, Placoderms, and Agnathans . . . . 81

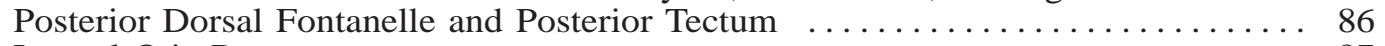

Lateral Otic Process . . . . . . . . . . . . . . . . . . . . . . . . . . . 87

Hyomandibular Fossa $\ldots \ldots \ldots \ldots \ldots \ldots \ldots \ldots \ldots \ldots \ldots \ldots \ldots \ldots \ldots$

Occipital Arch, Otico-Occipital Fissure, and Hypotic Lamina . . . . . . . . . . . . 89

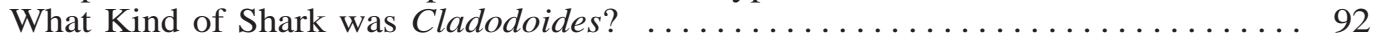

Phylogenetic Patterns in Paleozoic Sharks $\ldots \ldots \ldots \ldots \ldots \ldots \ldots \ldots \ldots \ldots \ldots \ldots \ldots . \ldots 3$

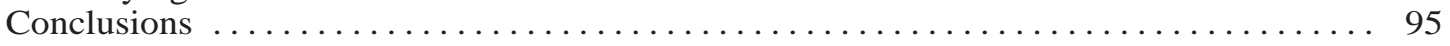

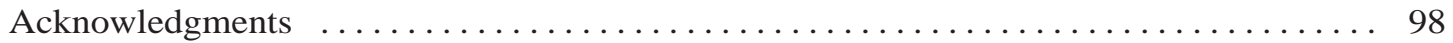

References $\ldots \ldots \ldots \ldots \ldots \ldots \ldots \ldots \ldots \ldots \ldots \ldots \ldots \ldots \ldots \ldots \ldots \ldots \ldots \ldots \ldots \ldots \ldots . \ldots . \ldots \ldots$ 


\begin{abstract}
The braincase of an Upper Devonian shark (Cladodoides wildungensis) is investigated using high-resolution CT scanning, and its internal and external morphology is described from threedimensional digital reconstructions. Many features are shared with modern elasmobranchs (neoselachians); these mostly represent conserved elasmobranch, chondrichthyan, or gnathostome characters. C. wildungensis resembles neoselachians in having a closed metotic fissure defining a glossopharyngeal canal, unlike some early chondrichthyans (e.g., Pucapampella, Orthacanthus) in which an open metotic fissure extends a considerable distance beneath the otic capsule. However, the glossopharyngeal canal in $C$. wildungensis is connected to a persistent dorsal fissure between the occipital arch and otic capsules, whereas this fissure becomes closed during neoselachian ontogeny. The embryonic polar cartilage apparently made a much greater contribution to the basicranium than in neoselachians and gnathostomes generally. This has affected the position of the efferent pseudobranchial foramen and the location of the orbital articulation in Cladodoides (both of which are located anteriorly in the orbit), as well as the size and extent of the dorsum sellae and the hypophyseal chamber inside the braincase. Another unusual feature in Cladodoides is the postorbital position of its trigeminal and facial nerves; instead of being situated in a trigemino-facial fossa of the orbit as in neoselachians, their foramina are located behind the postorbital process, although the pituitary vein and the origin of the external rectus eye muscle (located within the trigemino-facial fossa in neoselachians) both lie in the posterior part of the orbit. The oblique orientation of the postorbital arcade (derived from the embryonic lateral commissure) may be related to the exclusion of the trigeminal and facial nerves from the orbit. A laterally oriented canal through the process probably contained the trigeminal maxillary ramus (perhaps accompanied by the buccal ramus of the anterodorsal lateral line nerve). By contrast, in neoselachians these rami pass directly into the orbit (sometimes forming a buccal-maxillary complex), but they do not pass through the postorbital process. The Cladodoides braincase probably represents a juvenile or submature individual and shows indications of incomplete development (e.g., presence of a wide buccohypophyseal fenestra and absence of a precarotid commissure; incomplete enclosure of the internal carotids and orbital arteries by basicranial cartilage; incomplete closure of the braincase roof along the dorsal midline). The braincase also lacks multiple-layered prismatic calcification, possibly representing another juvenile feature. The phylogenetic relationship of $\mathrm{Cla}$ dodoides wildungensis is discussed. Dental, postcranial, and cranial features lend some support to the old hypothesis that cladodont sharks form a monophyletic group. While such a view is at variance with several recently published phylogenetic analyses, many of the characters noted in the present work have yet to be tested within a rigorous cladistic framework, and their phylogenetic significance is uncertain.
\end{abstract}

\section{INTRODUCTION}

Literature on the phylogeny of early gnathostomes is dominated by descriptions and comparisons of osteichthyans, placoderms, and acanthodians, but the body of literature concerned with fossil chondrichthyan anatomy is comparatively small. Despite this paucity of reliable morphological data (or perhaps because of it), chondrichthyans have sometimes been credited with considerable evolutionary importance (for example as putative placoderm descendants; Holmgren, 1942; Stensiö, 1969), while at other times they have been treated rather summarily, as a primitive sister group to osteichthyans (Rosen et al., 1981; Gardiner, 1984a). Invariably, however, the morphology of modern chon- drichthyans and elasmobranchs has helped steer these hypotheses, whereas little attention has been paid to extinct chondrichthyans. This stasis undoubtedly results from the great rarity of morphologically informative chondrichthyan fossils, as well as from inadequate preservation and a limited ability to observe crucial features even in better specimens. Whatever the reason, there has never been a comprehensive survey of morphological diversity in early chondrichthyans.

We need to look for more shark fossils, and the importance of new discoveries is amply illustrated by recent discoveries of articulated and other well preserved remains of Lower and Middle Devonian chondri- 
chthyans (e.g., Pucapampella, Doliodus, and Gladbachus; Maisey, 2001b; Maisey and Anderson, 2001; Heidtke and Krätschmer, 2001; Miller et al., 2003). However, we also need to utilize new technologies such as CT scanning to retrieve previously inaccessible morphological data from specimens we already have. From such studies, we have already learned that cranial morphology in early chondrichthyans differed in many respects from that in modern forms (Maisey, 2004a), requiring some radical reappraisal of character polarity not only in chondrichthyans, but in osteichthyans and even gnathostomes in general. For example, it is no longer possible to defend a relationship between placoderms and elasmobranchs on the basis of labyrinth morphology (Maisey, 2001a), or to regard the palatobasal articulation or ventral otic fissure as osteichthyan synapomorphies (paradoxically, the number of supposedly basal osteichthyan synapomorphies has fallen as our knowledge of early chondrichthyans has improved).

Modern elasmobranchs and holocephalans are regarded here as monophyletic sister groups within the crown group Chondrichthyes, and both possess autapomorphic traits suggesting they are divergently specialized. Thus, a modern shark such as Squalus is no more representative of elasmobranchs in general than a cow is of tetrapods; both retain conserved features of their respective groups, and both also possess an overlay of subsequently acquired apomorphic characters, but those in cows (e.g., as amniotes, therians, ungulates, etc.) have traditionally been more clearly identified than those of modern elasmobranchs. With an improved knowledge of the selachian fossil record, however, we are slowly gaining some insight into primitive elasmobranch morphology and how this may have changed during their subsequent evolutionary history.

The present investigation involves a shark braincase from the Upper Devonian limestone of Wildungen, Germany (fig. 1), first described and referred to Cladodus wildungensis by Gross (1937). The specimen has received considerable attention elsewhere (e.g., Holmgren, 1941; Schaeffer, 1967, 1981; Moy-Thomas and Miles, 1971; Jarvik 1980), and the reconstruction of the cranium and jaws presented by Gross (1938: fig. 2) will be familiar to many readers. However, the braincase was never completely prepared and its occipital region has only recently been revealed by CT scanning (Maisey, 2001a). This paper presents a revised and more complete account of cranial morphology in this specimen, drawing heavily on CT scanning and digital imaging. New data are presented which should be useful to investigators of other early chondrichthyans such as Gladbachus and Doliodus, and also to those interested in the interrelationships and early radiations in gnathostomes.

\section{MATERIALS AND METHODS}

The specimen under investigation (Forschungsinstitut und Naturmuseum Senckenberg, Frankfurt, P2468) is a late Devonian (Upper Frasnian) shark braincase, from the Wildungen Limestone, Wildungen, Germany (for a modern stratigraphic summary of this unit, see Maisch, 1998). It is almost perfectly preserved, with little distortion and minimal damage (fig. 1). External features of the specimen were described in considerable detail by Gross (1937), and several of his annotated line drawings are reproduced here as a single composite illustration, along with their original German annotations (fig. 2). Readers may find it useful to refer to this figure throughout the work.

It is important to note that the braincase described by Gross (1937) is not the holotype of $C$. wildungensis. That taxon is founded on jaw elements associated with teeth (Jaekel, 1921), and while Gross (1937) presented a circumstantial case for including the braincase in this species, no diagnostic characters can actually be compared. Thus, the precise identity of the braincase is problematic, despite its importance as a source of data concerning early elasmobranch cranial morphology. Moreover, many nominal species of Cladodus (including the type species, C. mirabilis Agassiz, 1843) are founded on isolated teeth, but Cladodus-like teeth occur in several different kinds of Paleozoic "cladodont" sharks including Cladoselache, stethacanthids, symmoriids, and "ctenacanths". This led Zangerl (1973) to declare Cladodus a nomen vanum (subsequently suppressed in 


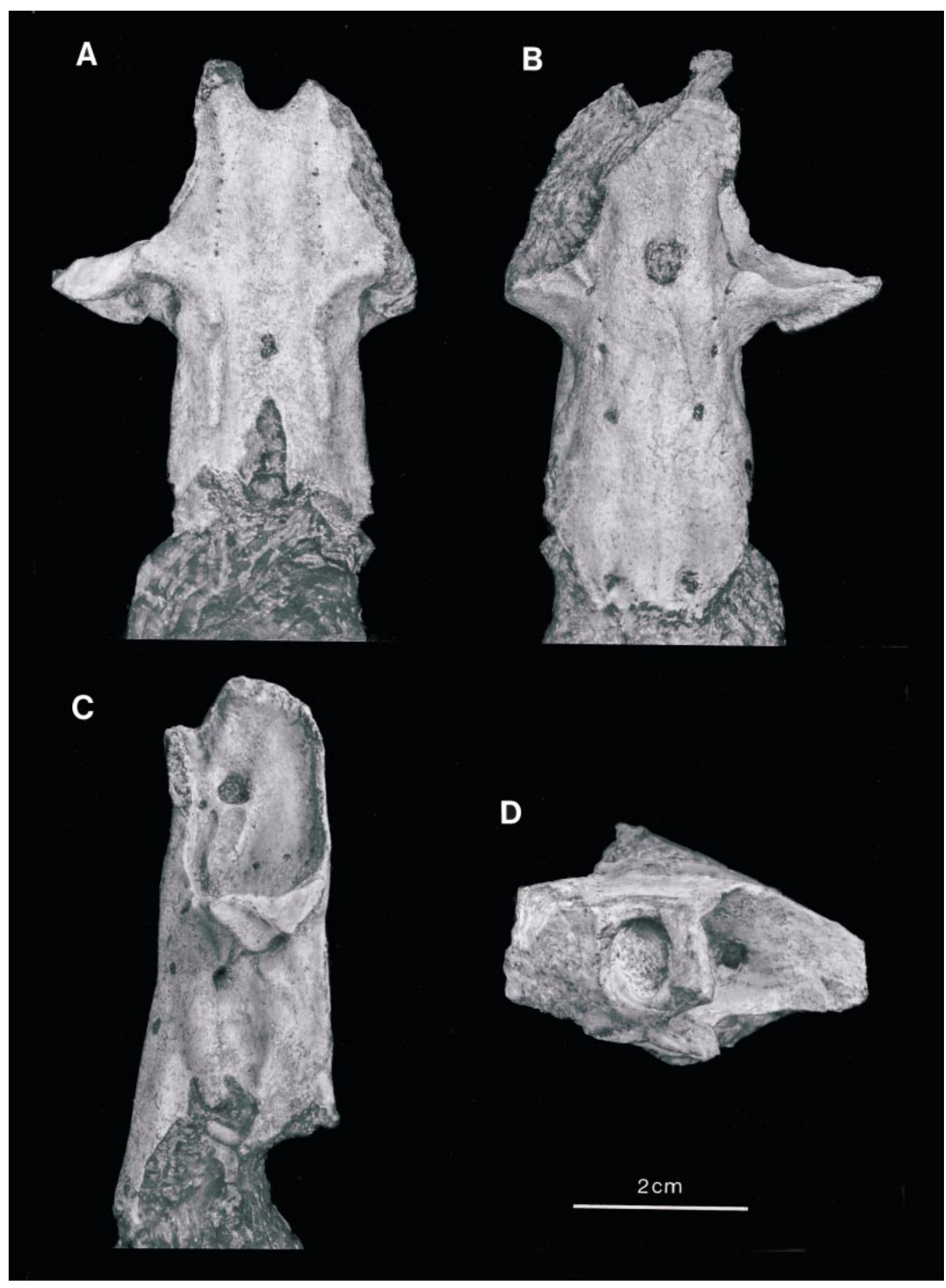

Fig. 1. Views of the Cladodoides braincase. (A) Dorsal view; (B) ventral view; (C) lateral view; (D) anterior view. 

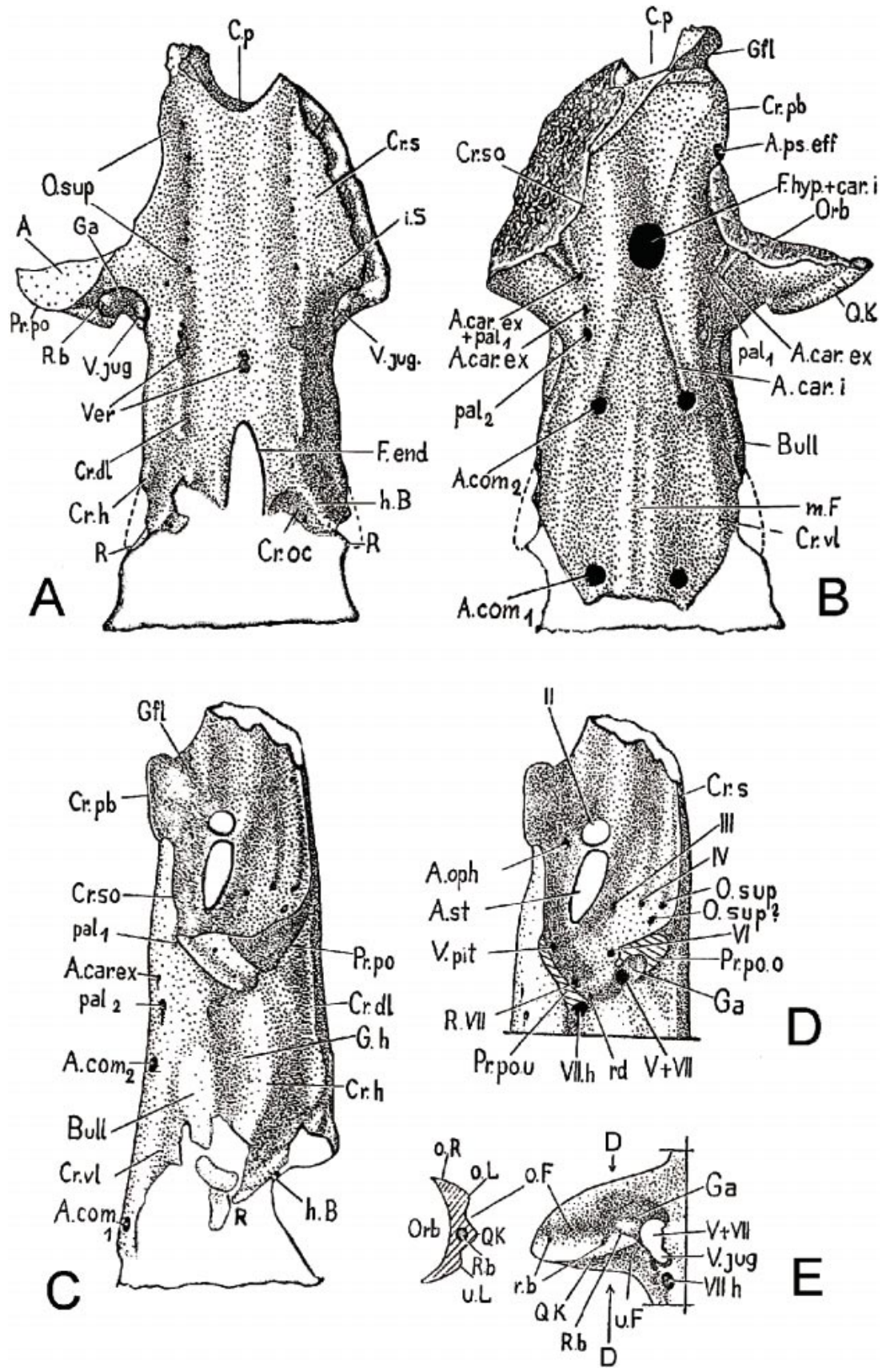

Fig. 2. Reproductions of original illustrations of the Cladodoides braincase, slightly modified from Gross (1937) by combining several originally separate figures. (A) Dorsal view; (B) ventral view; (C) left lateral view; (D) scrap lateral view of orbit with postorbital process removed; (E) posterior view of postorbital process. For the convenience of the reader, the original abbreviations of the annotations in German are retained, so that features identified by Gross and discussed in the text are more readily identified; for further explanation, the original work should be consulted. These annotations differ from those used elsewhere in the present paper and are not explained in the list of abbreviations. The only other changes from the original published drawings are digital clarification of leader lines and some abbreviations, cleaned outlines, and improved contrast. 
favor of the term nomen dubium; Chorn and Whetstone, 1978; Williams, 1985). Some new genera have been founded on specimens in which "Cladodus" teeth are associated with anatomically informative skeletal remains (e.g., Gutturensis Sequeira and Coates, 2000: formerly Cladodus neilsoni Traquair, 1898). Cladodus wildungensis was referred to a new genus Cladodoides by Maisey (2001a), but this reassignment does little to resolve its relationships since the distinction rests mainly on rather slight differences in the teeth of Cladodus mirabilis and $C$. wildungensis (e.g., the number and arrangement of lateral cusps). Nevertheless, dental characters do provide a basis for distinguishing some cladodont sharks (Ginter and Ivanov, 1992; Ginter, 1998 and in prep.), and the genus Cladodus may therefore eventually regain systematic utility. For example, Cladodus mirabilis teeth resemble those of the ctenacanth sharks from the Cleveland Shale in the morphology of articular buttons on the lingual surface of the basal platform and in the relationship of successional teeth in each tooth family. This tooth gestalt differs from that found in symmoriids and stethacanthids and may reflect a basic dichotomy within cladodont sharks. Unfortunately, the morphology of the basal platform is poorly known in Cladodoides wildungensis.

The specimen underwent considerable mechanical preparation prior to its original description, and in view of its historic and morphological importance no further preparation could be undertaken. Although the specimen is extremely informative, there are limitations on what can be observed externally. Computerized tomography (CT scanning) provides an ideal noninvasive procedure to investigate aspects of the fossil that are otherwise inaccessible and also provides a useful test of earlier observations. A high-resolution CT scan of the specimen was made at the Geology Department of the University of Texas at Austin (UTA). Scan parameters are as follows: $180 \mathrm{kV}, 0.133 \mathrm{~mA}$, no filter, glass wedge, $160 \%$ offset, slice thickness 5 lines (=0.262 mm), S.O.D. $92 \mathrm{~mm}, 2400$ views, 2 samples per view, interslice spacing 4 lines $(=0.2096 \mathrm{~mm})$, field of reconstruction 43.2 $\mathrm{mm}$, reconstruction offset 750 , reconstruction scale 33, scanned in three-slice mode.
Preliminary animated image processing at UTA used Voxblast to produce three-dimensional resliced renderings, in which slices are sequentially removed along the orthogonal $X-Y-Z$ axes while retaining the same slice thickness generated in the original scan imagery. Subsequent image processing by the author utilized Imaris/Surpass surface- and volume-generating software, by means of which structural contours were plotted on each original 8-bit CT scan slice. Three-dimensional isosurfaces and volumes of both the braincase and its endocast were created digitally from these contours, and individual images were captured in either isometric or perspective mode.

Two main kinds of three-dimensional images are generated by the Imaris/Surpass software, namely surface and volume renderings. A surface rendering is essentially a digital reconstruction of an object and is the three-dimensional equivalent of a drawing, whereas a volume rendering is more the scanning equivalent of a low-resolution photograph. Volume renderings are produced directly from a stack of original CT scan slices (transverse in this case) and provide an immediate three-dimensional rough image of the specimen, but internal features can only be seen on a slice-by-slice basis. However, surface renderings are calculated from contours plotted manually on each original slice, and points are then triangulated between adjacent slices by the computer, generating a geodesic surface which is then digitally filtered and smoothed. Surface renderings are time-consuming to produce but create remarkably detailed images which can be viewed in any orientation and in either perspective or standard orthographic projections. More important, internal features otherwise obscured by matrix and overlying structures are revealed by surface renderings. Surface renderings can also be sectioned by selecting an appropriate clipping plane, which permits structures to be viewed in almost any cross section (as in the series of slices through the postorbital process illustrating this work). Virtually any clipping plane can be generated by selecting different coordinates along the $X-Y-Z$ axes of the object, and the plane can also be tilted obliquely by rotating each axis independently. 

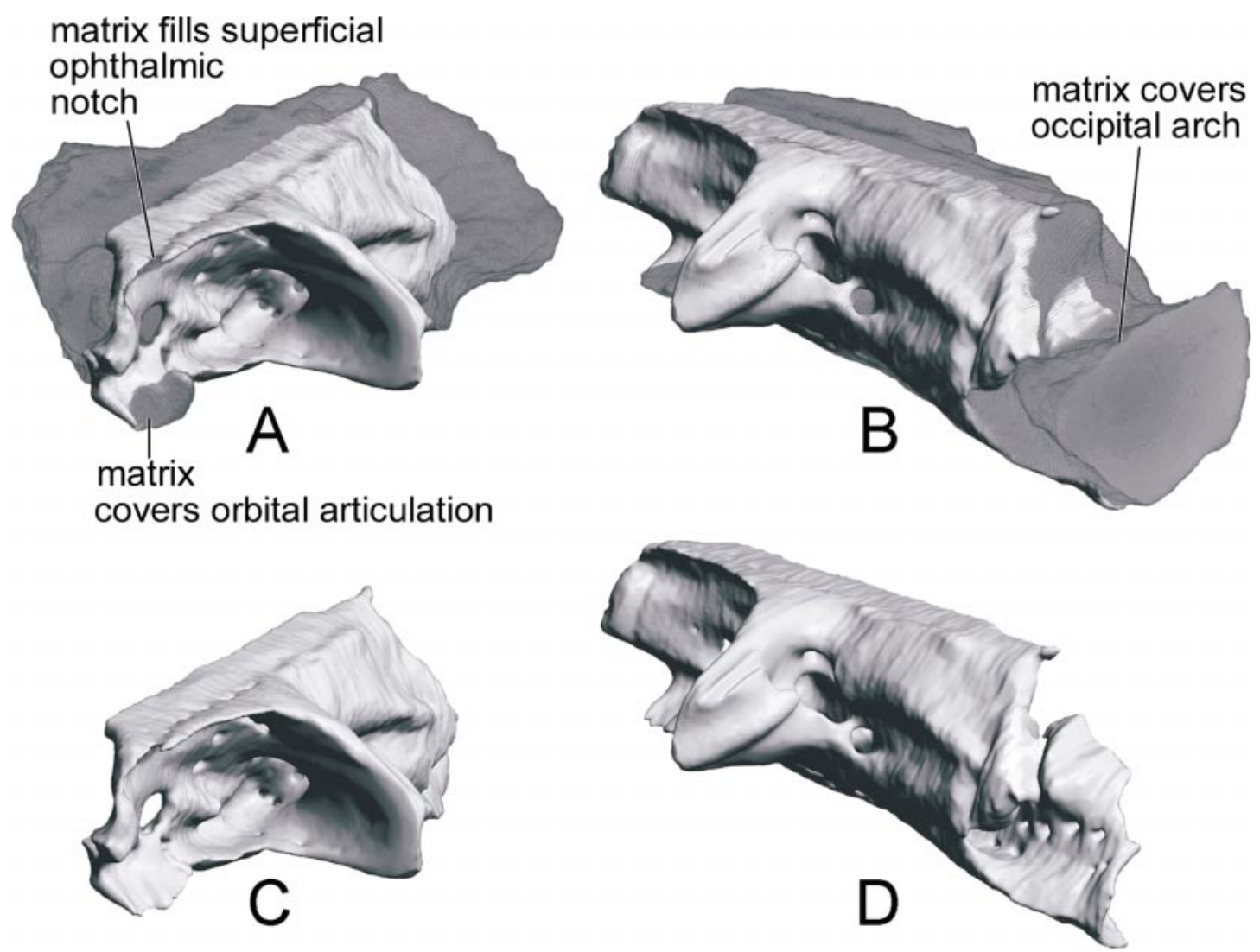

Fig. 3. Volume and surface renderings of the Cladodoides braincase. (A, B) Combined volume and surface renderings; (C, D) surface rendering only. Panels A and $\mathrm{C}$ are oblique left anterolateral views; panels B and D are oblique left posterolateral views. Note the marked lack of preparation in the occipital region and a thin but significant covering of matrix in the ethmoid region and the anterior part of the supraorbital shelf (darker gray areas).

Many of the illustrations in this work represent views captured from Imaris/Surpass surface renderings and processed using commercially available imaging software such as Adobe Photoshop. Only the left side of the braincase was used to generate contours, since it is more complete. The views of the complete braincase are composites, made by mirror-imaging the surface rendering with an appropriate allowance for different illumination of each side.

It is sometimes useful to combine volume and surface renderings although most morphological features are best revealed by the latter. Discrepancies between volume and surface renderings are easily identified by combining them in a single image, which provides a valuable aid during the initial in- terpretation of scans and the plotting of contours. Combining volume and surface renderings also reveals interesting taphonomic and forensic details about the Cladodoides braincase. Forensic observations include: the lack of original preparation in the occipital region; a thin layer of matrix covering the anterior palatoquadrate articulation, which was incorrectly considered to be broken by Gross (1937); and incomplete preparation and cleaning of the supraorbital shelf and other delicate structures (fig. 3). Such forensic observations illustrate the great value of CT scans in evaluating the accuracy of earlier reconstructions of fossils, particularly where further preparation is inadvisable or prohibited.

Another less complete shark braincase 
from the Devonian of Wildungen is housed in the Humboldt University Museum (Berlin). This specimen was described by Stensiö (1937), who referred it to Cladodus wildungensis. His paper actually appeared first, giving Gross (1937) an opportunity to dispute the identification and refer Stensiö's specimen to a new species, $C$. hassiacus. The two specimens are superficially similar but differ in size and matrix lithology, suggesting that they may have come from different localities and/or stratigraphic horizons. Schaeffer (1981: 42) found the morphological differences between the two specimens "mostly trivial or difficult to evaluate". However, now that the internal morphology of the Senckenberg specimen is known, profound differences from Stensiö's (1937) description of the otic region have emerged, although in many other respects the two specimens seem similar. Therefore, either Stensiö's (1937) interpretation of the otic capsule in the Berlin specimen is inaccurate, or two radically different taxa are represented by the Senckenberg and Berlin specimens. In this paper, only the Senckenberg specimen is referred to Cladodoides, pending a review of Stensiö's specimen.

Throughout this work, comparisons are made with braincases of other Paleozoic sharks, especially those referred to Tamiobatis and Xenacanthus by Romer (1964) and Schaeffer (1981). There is little doubt that all the Permian specimens from Texas and Oklahoma referred to Xenacanthus by Schaeffer (1981) represent xenacanth sharks, as these are by far the most abundant elasmobranch remains in those Permian redbeds. Unfortunately, most of these skeletal elements are isolated (apart from the spectacular specimen collected by A.S. Romer and illustrated by Schaeffer, 1981: fig. 1). However, these are very similar to more complete articulated fossils from Europe referred to Orthacanthus (e.g., O. senckenbergianus), and much of the American xenacanth material should be referred to Orthacanthus rather than Xenacanthus (e.g., $O$. texensis; Heidtke, 1998, 1999; Johnson, 1999).

Unfortunately, the systematic status of Tamiobatis is poorly resolved, although for different reasons than for Cladodoides. Tam- iobatis vetustus was erected originally by Eastman (1897), but his description is highly erroneous. The type specimen (NMNH 1717) is an isolated braincase, which lacks unambiguous diagnostic characters although it carries the taxon name. The specimen was collected in Powell County, Kentucky, but its exact provenance and horizon are uncertain. Romer (1964) and Schaeffer (1981) considered it to be early Mississippian in age, but Williams (1998) suggested that it may be late Devonian. When the specimen was originally collected, it was embedded in a limestone nodule with only its ventral surface exposed (identified as dorsal by Eastman, 1897), but it was entirely removed from the matrix by acid preparation prior to Romer's (1964) description. While this facilitated its investigation, apparently no matrix residues were retained, so samples are now unavailable for micropaleontological determination.

Schaeffer (1981) referred an isolated braincase from the early Mississippian of Bedford (Indiana) to "Tamiobatis sp." (AMNH 2140). Subsequently, Williams (1998) referred a more complete fossil (CMNH 9280, including a braincase, jaws, teeth, denticles, and the impression of a finspine) from the late Devonian Cleveland Shale of Bedford (Ohio) to the type species $T$. vetustus. However, the emended diagnosis provided by Williams (1998) consists only of dental characters, which are inappropriate both for the genoholotype NMNH 1717 and for AMNH 2140. Thus, three specimens from differing horizons and localities have now been referred to Tamiobatis, yet there are still no reliable criteria to distinguish the holotype of the type species from other $\mathrm{Pa}$ leozoic sharks with similar cranial morphology (e.g., Ctenacanthus). Furthermore, while the braincases CMNH 9280 and NMNH 1717 are generally similar, the arrangements of their basicranial foramina differ slightly, suggesting that they may represent different taxa. Williams (1998) compounded the problem further by referring additional species (all founded on isolated teeth) to the genus Tamiobatis (T. succinctus, wachsmuthi, and springeri). While all the material investigated by Williams (1998) could represent a distinct genus based on dental morphology alone, its inclusion in Tamiobatis is unjustified and 
cannot be supported by cranial synapomorphies with NMNH 1717. Thus, there is no compelling evidence that the three braincases already referred to Tamiobatis are really congeneric, although there is every reason to suppose they pertain to quite similar sharks, given their overall morphological similarity. In the absence of generically diagnostic characters for Tamiobatis, the genus should probably be confined to the holotype of the type species, NMNH 1717.

Comparison of Cladodoides with other Paleozoic sharks is limited by paucity of data, especially concerning internal features of the braincase (these have only been described in two other forms, "Tamiobatis sp." and Orthacanthus; Schaeffer, 1981). The isolated "Tamiobatis sp." braincase (AMNH 2140) is of importance to this work because its internal morphology is revealed in a series of horizontal slabs that can be compared with scans of Cladodoides. However, a detailed description is beyond the scope of this work, and only some of its features are discussed below. AMNH 2140 is far larger and more heavily calcified than the Cladodoides braincase. Features of cranial morphology in $\mathrm{Or}$ thacanthus are taken mainly from Schaeffer (1981) and Maisey (1983).

Recently, some fragmentary skeletal remains associated with teeth identified as $\mathrm{Cla}$ dodus elegans were discovered in the British Geological Survey collections (M. Ginter, personal commun., 2003). That material is not necessarily from a single individual and not all the fragments are associated with teeth, but all are from the Lower Carboniferous Dockra Limestone of Lugton Quarry, Ayrshire, Scotland. Teeth of C. elegans are very similar to those of $C$. mirabilis (the type species), and this discovery may therefore help clarify the systematic position of the genus. The remains (which will be described elsewhere) include: the midorbital region of a large braincase, with wide supraorbital crests and most of the orbital wall; a large central section of another braincase; a complete occipital arch, with the posterior part of the otic capsule and a broken lateral otic process; associated jaw elements, including the right palatoquadrate, Meckel's cartilage, and ceratohyal; and the anterior part of a left palatoquadrate, showing two or three depres- sions for tooth families. Cladodus elegans teeth are associated with the midorbital fragment and the isolated occipital region. These fragments are almost identical to the corresponding regions of the "Tamiobatis sp." braincase described by Schaeffer (1981), and the referred palatoquadrate is "cleavershaped", with a strong postorbital process bearing an articular surface (like the ctenacanth palatoquadrate described by Williams, 1998), suggesting that the cranial endoskeletons of Cladodus elegans (and C. mirabilis?), Tamiobatis vetustus, "Tamiobatis sp.", and Cladodoides were very similar.

At various places in the text, references are made to an isolated symmoriid braincase ("Cobelodus") from the Pennsylvanian Fayetteville Shales of Arkansas (Maisey, 2004a); a detailed description of this specimen is still in preparation. In addition, Schaeffer (1981) tentatively referred three specimens with braincases from the Cleveland Shale to Ctenacanthus (CMNH 5965, 6919, 7852), but these specimens have not yet been investigated or described and they are omitted from further discussion here.

\section{ANATOMICAL ABBREVIATIONS}

\begin{tabular}{|c|c|}
\hline aa & anterior ampulla \\
\hline acv & foramen for anterior cerebral vein \\
\hline adll & $\begin{array}{l}\text { passage for anterodorsal lateral line } \\
\text { nerve }\end{array}$ \\
\hline appr & anterior postorbital process (arthrodire) \\
\hline asc & anterior semicircular canal \\
\hline avll? & $\begin{array}{l}\text { passage for anteroventral lateral line } \\
\text { nerve }\end{array}$ \\
\hline $\mathrm{cc}$ & crus commune \\
\hline cer & cerebellar region \\
\hline dor & dorsal otic ridge \\
\hline ds & dorsum sellae \\
\hline ea & external (horizontal) ampulla \\
\hline ebra1 & efferent branchial artery \\
\hline ha & dean artery \\
\hline epsa & efferent pseudobranchial artery \\
\hline end $f$ & endolymphatic foramen \\
\hline esc & external (horizontal) semicircular canal \\
\hline $\mathrm{fm}$ & foramen magnum \\
\hline $\mathrm{g}$ & median basicranial groove \\
\hline gc & glossopharyngeal canal \\
\hline gr prof? & groove for profundal nerve \\
\hline hl & hypotic lamina \\
\hline hm art & position of hyomandibular articulation \\
\hline & hypophyseal chamber \\
\hline hyp $\mathrm{f}$ & bucco-hypophyseal fenestra \\
\hline ic a & internal carotid artery \\
\hline
\end{tabular}




\begin{tabular}{|c|c|}
\hline $\begin{array}{l}\text { iva } \\
\text { jc }\end{array}$ & $\begin{array}{l}\text { foramen for intervertebral artery } \\
\text { jugular canal }\end{array}$ \\
\hline lda & lateral dorsal aorta \\
\hline lhr & lateral hypophyseal ridge \\
\hline lof & lateral otic fossa \\
\hline lor & lateral otic ridge \\
\hline mdv? & inferred position of mandibular vein \\
\hline med & medullary region \\
\hline mes & mesencephalic region \\
\hline met $\mathrm{f}$ & metotic fissure \\
\hline & median suture in braincase roof \\
\hline not can & notochordal canal \\
\hline n soph & notch for superficial ophthalmic ramus \\
\hline oc a & occipital arch \\
\hline oc $\cot$ & occipital cotylus \\
\hline olf $\mathrm{c}$ & olfactory canal \\
\hline oof & otico-occipital fissure \\
\hline opa & optic (retinal) artery \\
\hline opha & ophthalmic artery \\
\hline ora & orbital artery \\
\hline $\begin{array}{l}\text { or art } \\
\text { pa }\end{array}$ & $\begin{array}{l}\text { orbital articulation of palatoquadrate } \\
\text { posterior ampulla }\end{array}$ \\
\hline pac & preampullary canal \\
\hline $\mathrm{pbc}$ & posterior basicapsular commissure \\
\hline pcc & precarotid commissure \\
\hline pdf & $\begin{array}{l}\text { posterior dorsal fontanelle }(=\text { parietal } \\
\text { or endolymphatic fossa of authors) }\end{array}$ \\
\hline ped & attachment area for optic pedicel \\
\hline pf? & possible pineal organ \\
\hline $\mathrm{p}$ fen & perilymphatic fenestra \\
\hline pit $\mathrm{v}$ & pituitary vein \\
\hline po art & $\begin{array}{l}\text { postorbital articulation for palatoquad- } \\
\text { rate }\end{array}$ \\
\hline popr & postorbital process \\
\hline pppr & posterior postorbital process (arthrodire) \\
\hline pref & precerebral fontanelle \\
\hline prfc & prefacial commissure \\
\hline prof & profundal nerve foramen \\
\hline psc & posterior semicircular canal \\
\hline psr & presphenoid ridge \\
\hline pt & posterior tectum \\
\hline re & insertion of external rectus muscle \\
\hline sac & saccular chamber \\
\hline snc & subnotochordal "chamber" \\
\hline sns & subnotochordal septum \\
\hline soc & $\begin{array}{l}\text { spino-occipital nerve canals (in the en- } \\
\text { docast) }\end{array}$ \\
\hline sof & spino-occipital nerve foramina \\
\hline soph & $\begin{array}{l}\text { superficial ophthalmic complex (tri- } \\
\text { geminal }+ \text { anterodorsal lateral line } \\
\text { nerves) }\end{array}$ \\
\hline sub s & suborbital shelf \\
\hline sup $\mathrm{s}$ & supraorbital shelf \\
\hline tel & telencephalic region \\
\hline ur & utricular recess \\
\hline vlc & vesticulolateral (auricular) chamber \\
\hline II & optic nerve \\
\hline III & oculomotor nerve \\
\hline
\end{tabular}

$\begin{array}{ll}\text { IV } & \text { trochlear nerve } \\ \text { V } & \begin{array}{l}\text { trigeminal nerve } \\ \text { Vandibular ramus of trigeminal nerve }\end{array} \\ \text { VI } & \begin{array}{l}\text { abducent nerve } \\ \text { facial nerve }\end{array} \\ \text { VII } & \text { anterior ramule of palatine ramus } \\ \text { VII ar } & \text { hyomandibular trunk of facial nerve } \\ \text { VII h } & \text { hyoidean ramus of facial nerve } \\ \text { VII hy } & \text { palatine ramus of facial nerve } \\ \text { VII pal } & \text { pasterior ramule of palatine ramus } \\ \text { VII pr } & \text { postaval (acousticovestibular) nerve } \\ \text { VIII } & \text { octaval glossopharyngeal nerve } \\ \text { IX } & \text { vagal nerve } \\ \text { X } & \end{array}$

Note that this list does not include abbreviations for the figures reproduced from Gross (1937) and Stensiö (1937); see captions for details. Terminology for cranial nerves here follows Northcutt and Bemis (1993) in recognizing a distinct series of octavolateral nerves (including the octaval and lateral line nerves), instead of including them as parts of the facial, glossopharyngeal, and vagal nerves. The profundal is also treated as a separate nerve, rather than a branch of the trigeminal nerve. All nerve names are anglicized.

\section{DESCRIPTION}

\section{General Features, Taphonomy, and FORENSIC OBSERVATIONS}

Comparison of the surface renderings presented below with the illustrations in Gross (1937; see fig. 2 here) shows that CT scanning has captured the entire morphology of the Senckenberg Museum braincase in great detail. Scanning provides information not only about the morphology of a fossil specimen, but also about its postmortem taphonomic history and its subsequent forensic history (preparation, conservation, damage and repair). Diagenetic observations of interest in the Senckenberg braincase include the lack of compression or subsequent distortion throughout the specimen; the presence of a complete occipital region still enclosed by matrix; the uniform carbonate matrix completely filling the braincase, leaving no voids, secondary crystalline areas, or pyrite overgrowths; and the absence of bioturbation. Forensic observations of interest include damage and slight repair to the right postorbital process (especially its tip), and unrepaired damage to the lateral walls of both otic cap- 


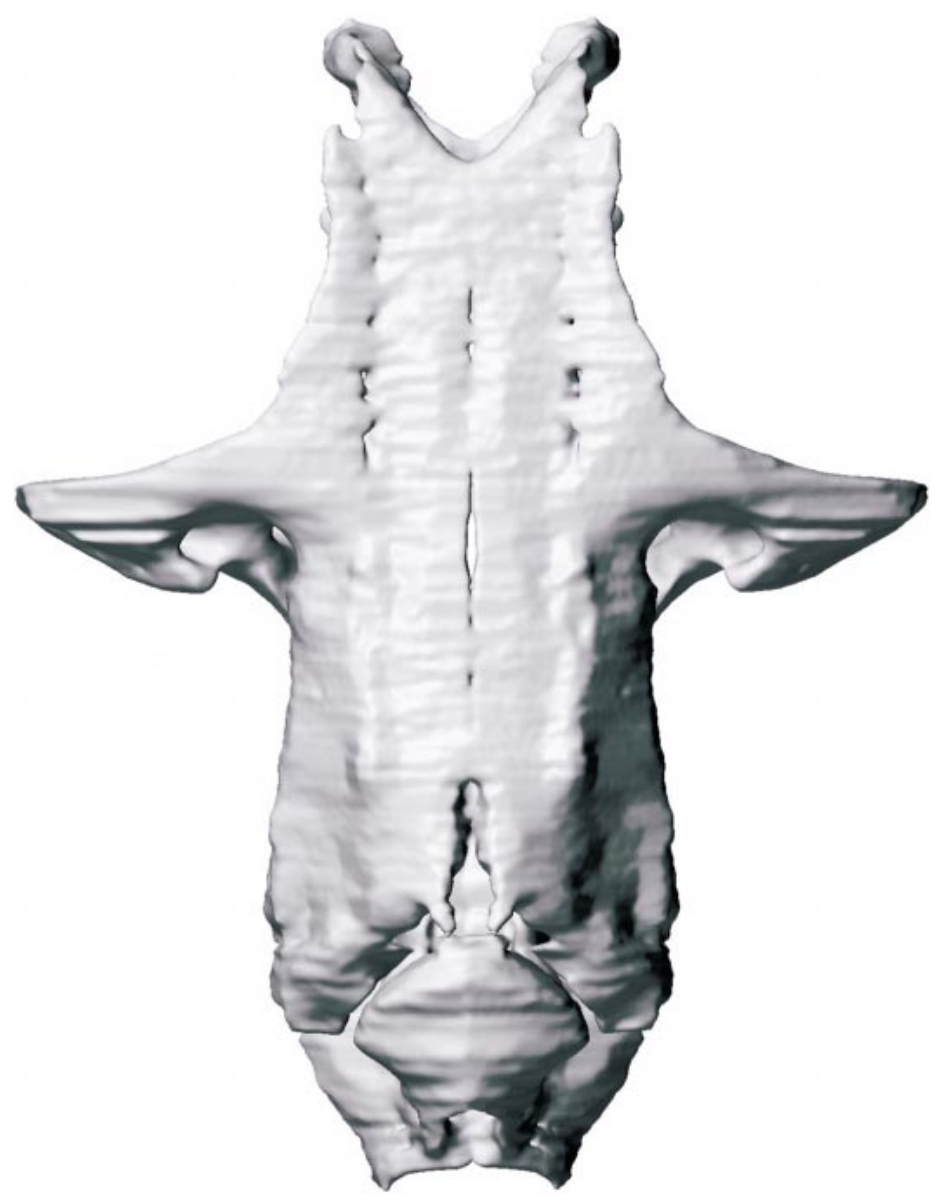

Fig. 4. Dorsal view of the Cladodoides braincase (surface rendering).

sules. There is no evidence of any dermal structures (teeth, denticles) or parts of the postcranial and visceral skeleton in the scanned matrix, and the braincase appears to be completely isolated. Only a very thin layer of prismatically calcified cartilage covered the inner and outer surfaces of the braincase, rather than heavy or multiple-layered calcification seen in other Paleozoic sharks such as Orthacanthus. The delicate nature of this specimen suggests it underwent very little postmortem disturbance or transportation. Gross (1937) noted that the jaws figured by Jaekel (1921) came from the same locality and are in similar matrix, suggesting that these specimens may pertain to a single taxon if not to one individual.

In dorsal view (fig. 4), the postorbital pro- cess separates the orbital region from the slightly longer otico-occipital region. Missing areas include most of the antorbital region (although the posterior part of the precerebral fontanelle is still present) and the lateral otic process farther posteriorly. These regions have not been reconstructed here. Anteriorly, combined surface and volume renderings reveal a matrix-filled notch (not observed by Gross, 1937) for the superficial ophthalmic ramus (fig. 3). The occipital arch is also covered by matrix in the specimen but is revealed by the scan and is clearly separated from the otic region by a persistent otico-occipital fissure.

In ventral view (fig. 5), the basicranium forms an unbroken surface, with no ventral otic fissure. Anteriorly, the floor of the eth- 


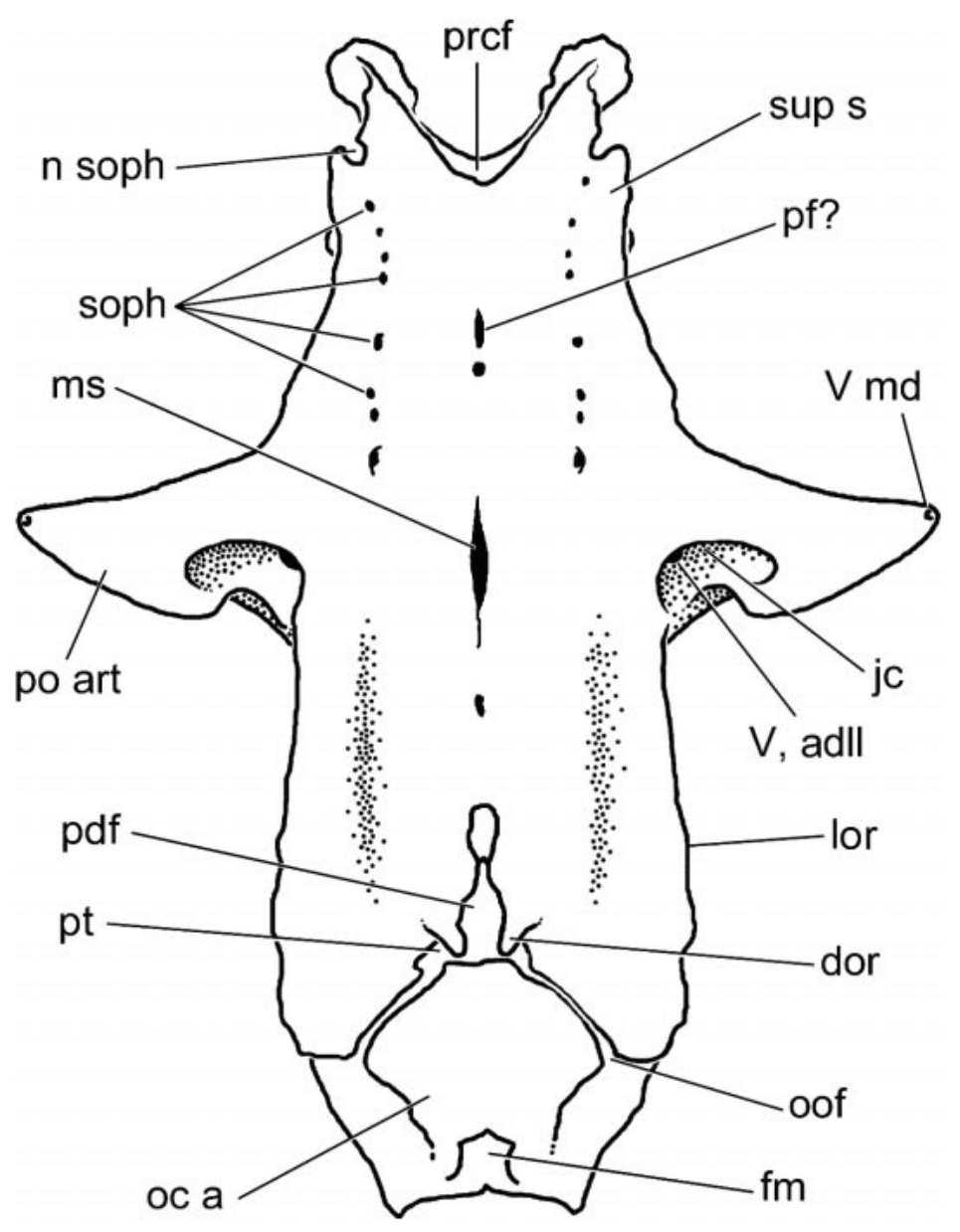

Fig. 4 Continued.

moid region is missing. There is a large bucco-hypophyseal fenestra leading into a matrix-filled central opening for the hypophyseal chamber.

The lateral view (fig. 6) shows the major foramina within the orbit and also reveals the occipital arch for the first time, including several foramina for spino-occipital nerves and intervertebral arteries. Features in the posterior part of the orbit are obscured in this view by the postorbital process but are illustrated later in this work.

The medial view (fig. 7) dramatically illustrates the investigative power of CT scanning in paleontology, by reconstructing the internal morphology of the braincase as if sliced through the sagittal plane. All the fea- tures exhibited here are obscured by matrix and have never before been observed. The internal openings of all the major cranial nerves and blood vessels can be recognized, and many details of labyrinth morphology are also evident. The dorsum sellae is particularly prominent, extending from below the oculomotor foramen and sloping posteriorly beneath the abducent foramen. A persistent otico-occipital fissure is also seen, separating the otic capsule from the lateral wall of the occipital region.

In the anterior and posterior views of the braincase (fig. 8) the dorsum sellae is again observed deep within the cranial cavity, forming a prominent transverse wall. Also visible are large openings in the orbital sur- 


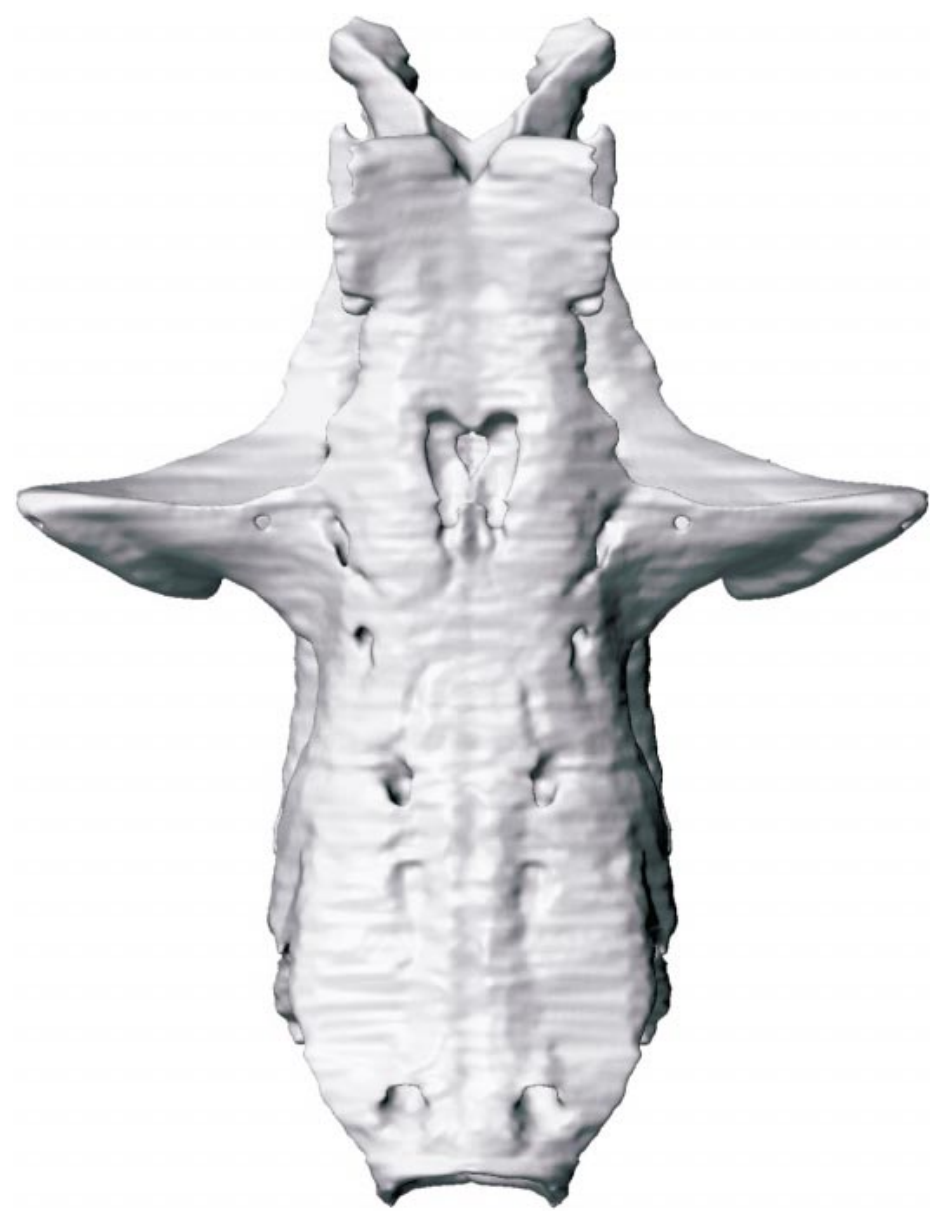

Fig. 5. Ventral view of the Cladodoides braincase (surface rendering).

face of the postorbital process; these are completely obscured by matrix and were not observed by Gross (1937) or Schaeffer (1981). The extent of the otico-occipital fissure is particularly evident in posterior view.

The braincase is quite small (its maximum length, including the unexposed occipital region, is approximately $65 \mathrm{~mm}$ ), but it is essentially intact and has suffered minimal distortion. Despite its small size, the braincase is calcified superficially (both internally and externally) with prismatic hard tissue (a chondrichthyan characteristic), although some regions may have been unmineralized, and internal features did not show up well locally because of poor X-ray density contrast between the phosphatic calcification of the braincase and the limestone matrix.
Throughout the braincase, the prismatic calcification is thin and there is no evidence of multiple layers, thickened regions of prismatic tissue, or calcified fibrocartilage. This observation is important, because Schaeffer (1981) claimed that multiple-layered calcification was one of the few characters putatively linking Cladodoides with Tamiobatis and Orthacanthus. If the Cladodoides braincase represents a juvenile or submature individual (a distinct possibility, discussed below), multiple-layered calcification may not yet have formed. Multiple prism layers have been reported in the braincase of a stethacanthid (Akmonistion; Coates and Sequeira, 1998), but they are not as extensive or thick as in Cladodus elegans, Tamiobatis, Orthacanthus, or ctenacanth sharks from the 


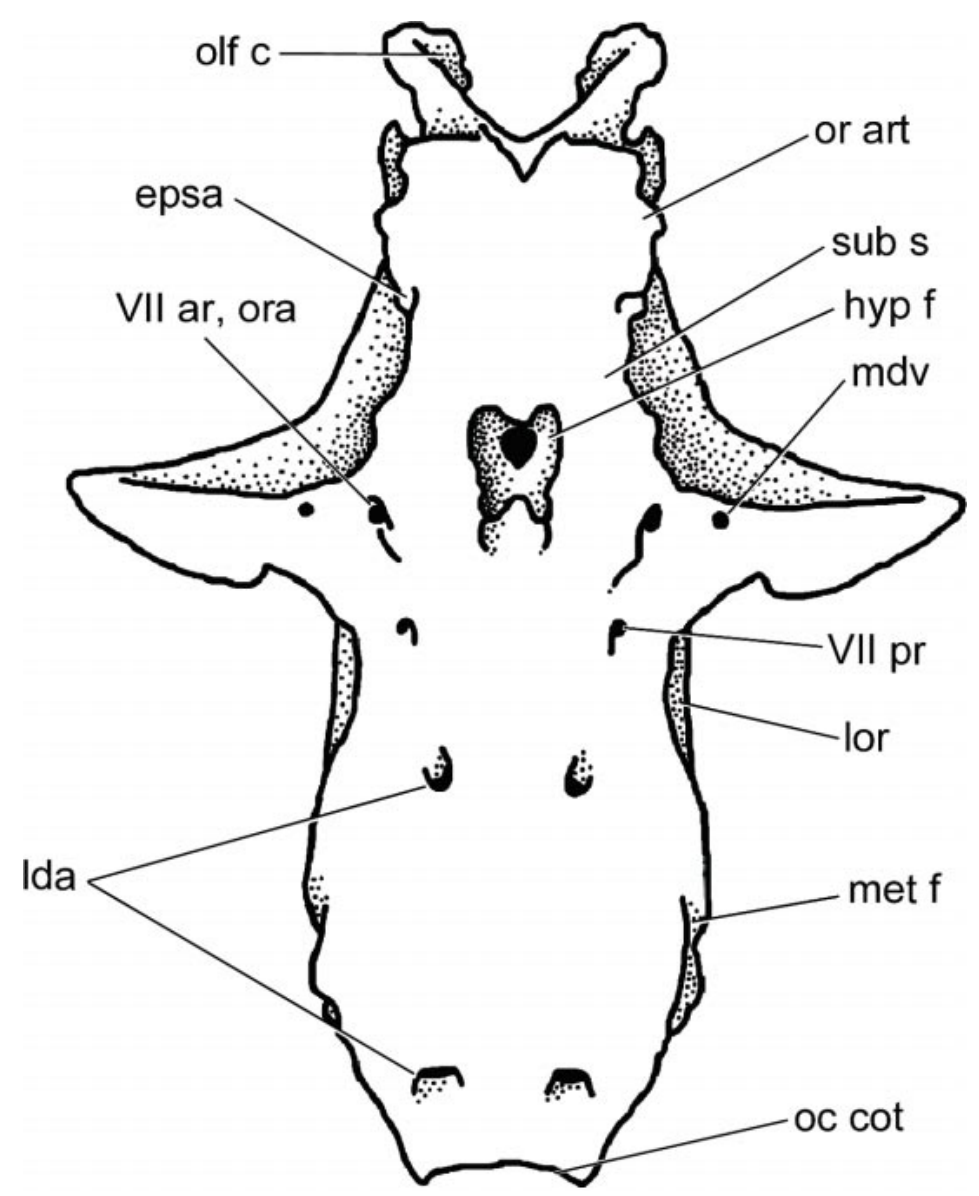

Fig. 5. Continued.

Cleveland Shale. Note that in cladoselachians from the Cleveland Shale the braincase is also thinly mineralized and less robust than in ctenacanths, although it has not yet been determined whether the prismatic calcification in cladoselachians consists of more than a single layer. The fragmentary cranial material from Scotland referred to Cladodus elegans has multiple-layered calcification.

EthmoID REgion: Almost all the ethmoid region is missing in the Senckenberg braincase, and only a small part of the left olfactory canal is recognizable. No parts of the postnasal wall, ectethmoid chambers, ethmoid and/or ectethmoid processes, orbitonasal canals, or olfactory capsules are preserved. The telencephalic region of the endocranial cavity extends between the orbits and is widest immediately below the optic foramen (fig. 7), forming a low presphenoid ridge or ledge (Praesphenoidvorsprung; Gegenbaur, 1872). This ridge is well developed in some neoselachians but is weak or absent in others (Maisey, 2004b).

There is no evidence of a ventral ethmoidal keel, although one may have been developed farther anteriorly. In Orthacanthus, the basicranium narrows abruptly in front of the anterior palatoquadrate articulation to form a narrow base for the rostrum (Schaeffer, 1981: figs. 5, 6). In Hybodus basanus, the basicranium is also narrowed anteriorly, forming a caudal internasal keel beneath paired ethmopalatine processes (Maisey, 1983: figs. 8, 9). In both those forms, the ethmoidal keel is confined to the region anterior to the optic foramen, whereas very little of this region is preserved in the Senckenberg braincase. 

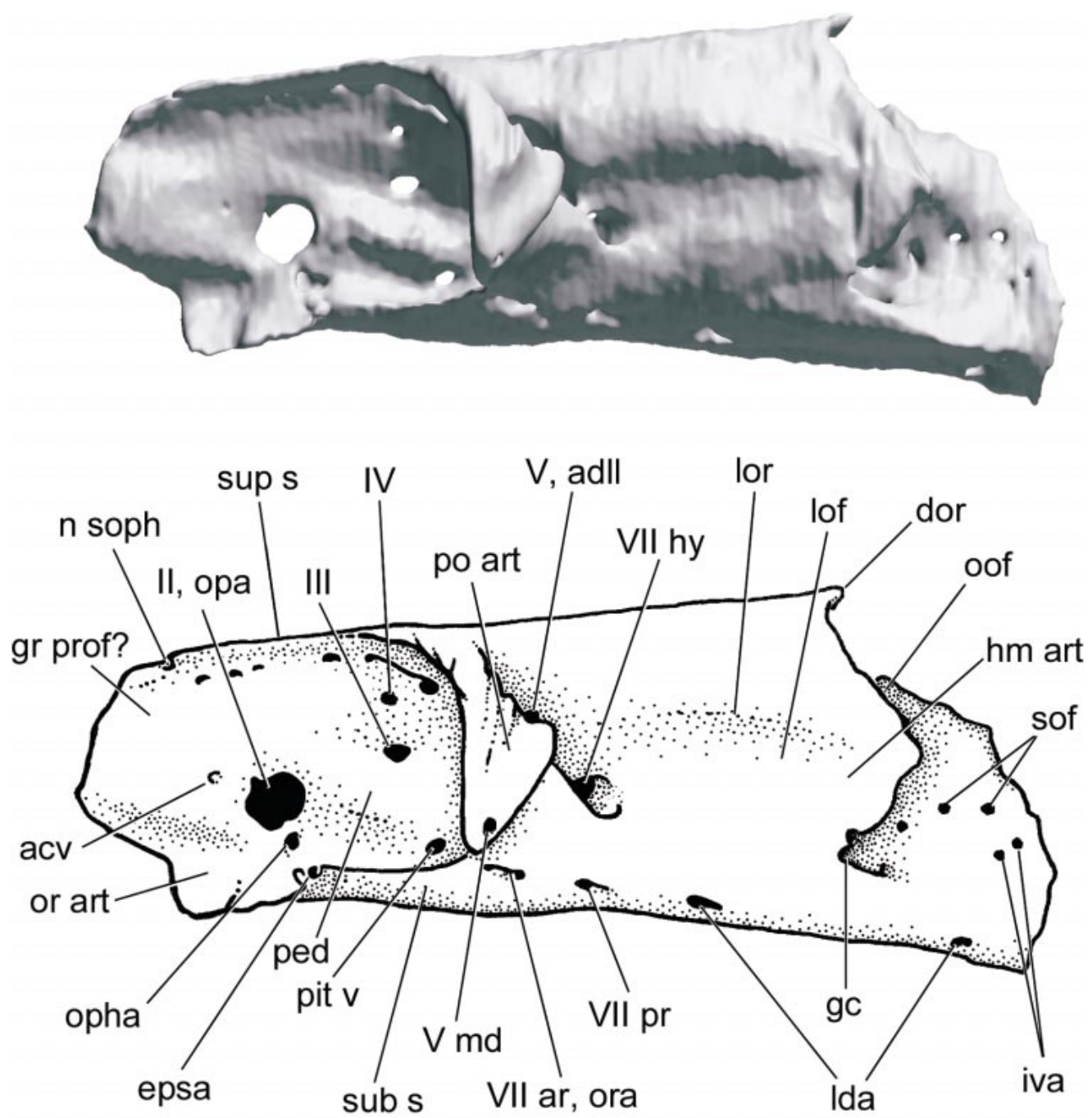

Fig. 6. Lateral view of the Cladodoides braincase (surface rendering).

Coates and Sequeira (1998) did not find any evidence of an ethmoidal keel in Akmonistion, but even in their most complete braincase the region anterior to their "posterior ethmoid process" is not preserved. The phylogenetic significance of the ethmoidal keel in elasmobranchs was recently reviewed elsewhere (Maisey, 2004b: 41) and will not be discussed here.

The entire preorbital region of the Humboldt University "Cladodus" hassiacus braincase is also missing, but Stensiö (1937: fig. 6) reconstructed it by superimposing the ethmoid region of the modern frilled shark
Chlamydoselachus (see also Holmgren, 1941: fig. 11). However, while Chlamydoselachus may occupy a basal position among neoselachians ( $=$ crown group elasmobranchs), there is no evidence that the taxon represented by the fossil was more closely related to Chlamydoselachus than to any other modern elasmobranch, and therefore such a reconstruction is not justified phylogenetically, although the general morphology of the ethmoid region may have been similar. In Chlamydoselachus, the floor of the telencephalic chamber slopes upward anteriorly and the floor of the precerebral fontanelle is el- 

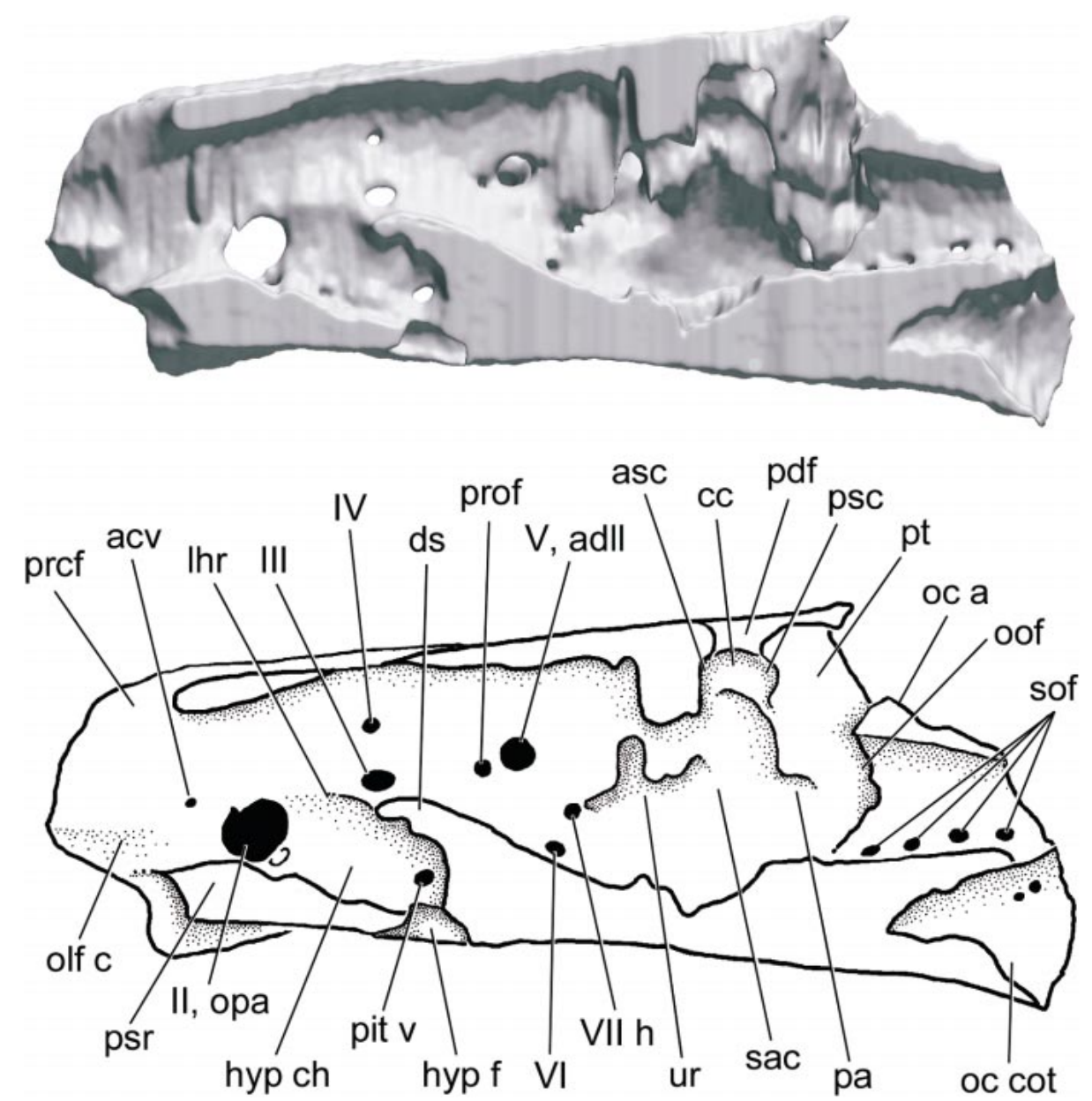

Fig. 7. Medial view of the Cladodoides braincase sliced through the sagittal plane (surface rendering).

evated almost to the same level as the roof of the braincase (Allis, 1923a; Maisey, 2004b). This arrangement is atypical of most neoselachians and may represent an autapomorphy of the genus. Judging from the depth of the preserved part of the telencephalic chamber in the Senckenberg braincase, its fontanelle floor was probably much deeper than in Chlamydoselachus.

As in elasmobranchs generally, the endocranial chamber in Cladodoides is open anteriorly, forming a large precerebral fontanelle that intrudes slightly between the olfactory canals as in Notorynchus (Maisey, 2004b). The exposed part of the fontanelle floor is smooth and confluent with the wall of the olfactory canal. Scanning shows that the unexposed floor of the precerebral region farther posteriorly is also smooth. The course of the terminal nerve is unknown.

The olfactory capsules and the antorbital (postnasal) wall are not preserved in the Senckenberg braincase, and its olfactory canals are represented only by a slight bulge in the lateral wall of the braincase just above the anterior palatoquadrate articulation. The olfactory tracts were probably extensive anteroposteriorly, as in neoselachians. In $\mathrm{Or}$ thacanthus, the lateral wall of the olfactory canal bulges strongly into the orbit behind the preorbital process (Schaeffer, 1981: fig. 2; Maisey, 1983: fig. 14B). In neoselachians, the olfactory canal is usually located medial to the main antorbital cartilage, which can 

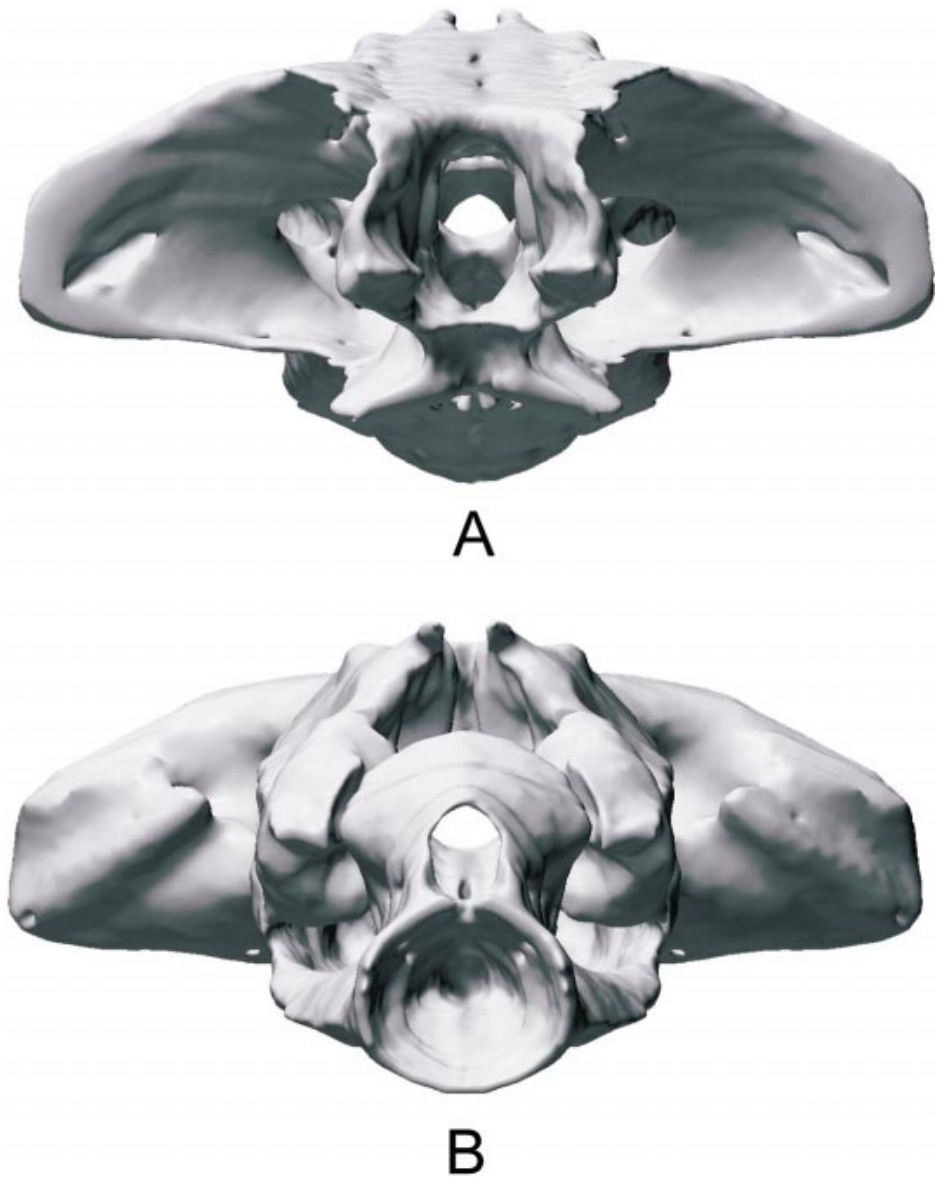

Fig. 8. Anterior (A) and posterior (B) views of the Cladodoides braincase (surface renderings).

form a prominent partition separating the orbit from the ectethmoid chamber.

Just anterior to the optic foramen is a very small canal (not detected by Gross, 1937), which may have contained the anterior cerebral vein, since this vessel occupies a similar position in neoselachians. The canal opens internally and cannot therefore have transmitted the profundal nerve onto the dorsal surface of the snout. Holmgren (1941) noted that the profundal nerve shares a common opening with the anterior cerebral vein in some specimens of Chlamydoselachus, but in others they are separate (as in other neoselachians). In Cladodoides, the profundal nerve probably left the orbit via a shallow groove dorsal to the olfactory canal, but no profundal foramen has been identified.

Extent of Polar Cartilage-Derived Re-
GION: In Cladodoides, there are separate foramina for the efferent pseudobranchial artery and the pituitary vein. These vessels therefore entered the braincase separately (as in most neoselachians, although they share an opening in some torpedo rays; Holmgren, 1941). In modern gnathostomes, these foramina are important ontogenetic landmarks, indicating the anterior and posterior limits of the polar cartilage, respectively (De Beer, 1931, 1937). In Cladodoides, the efferent pseudobranchial and pituitary foramina are spaced far apart, suggesting that the contribution made by the polar cartilage to the basicranium was extensive. This is also supported by the extensive dorsum sellae and hypophyseal chamber in the endocast (see below). If the positions of these foramina are reliable indicators of the original boundaries 


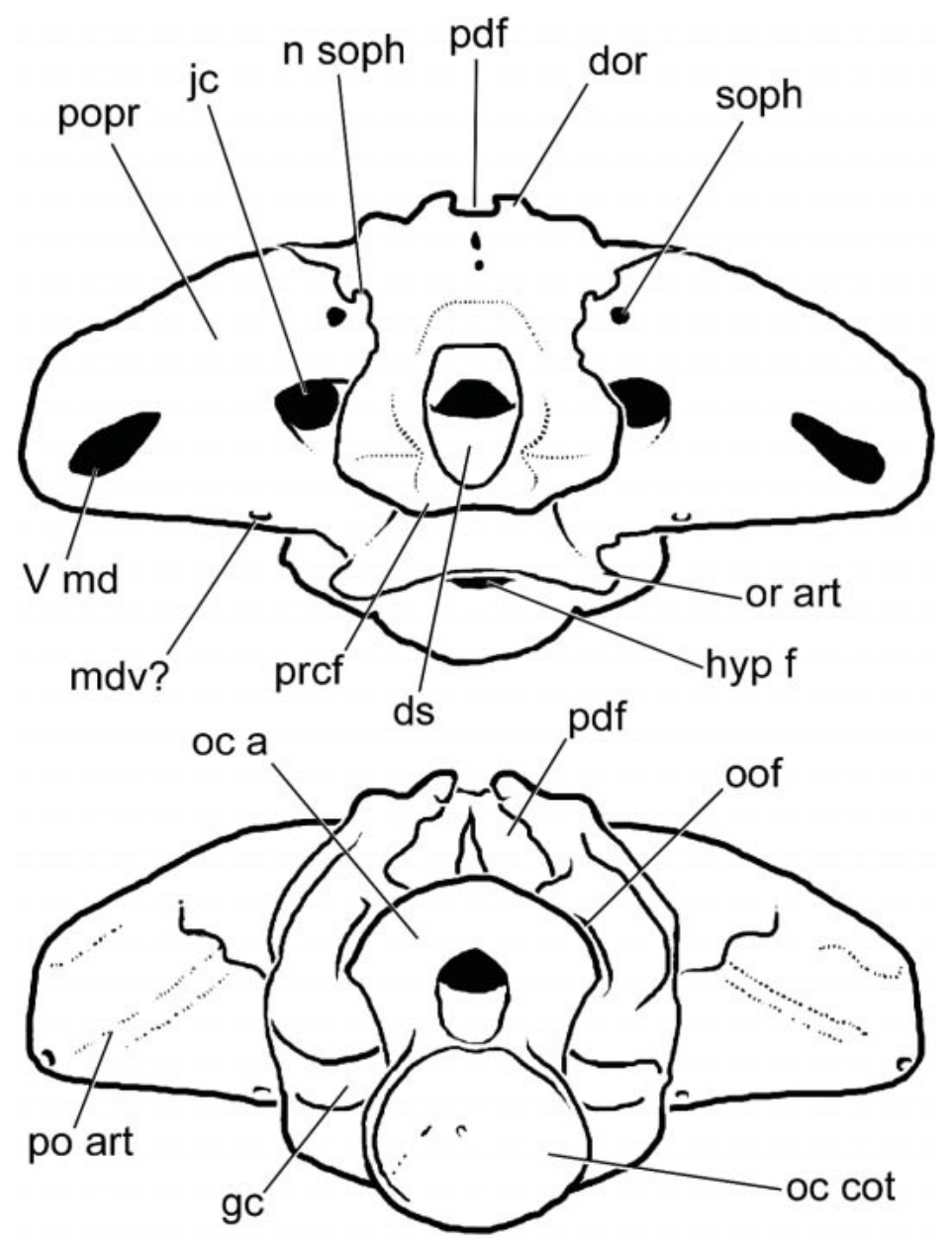

Fig. 8. Continued.

of embryonic tissues in Cladodoides, the polar cartilage formed perhaps half the orbital floor plus a considerable part of the basicranium (fig. 9), including an extensive postpituitary commissure that extended behind the internal carotids, and perhaps also forming the lateral walls of the bucco-hypophyseal fenestra. The area derived from the polar cartilage in Cladodoides presumably extends from the postpituitary commissure posteriorly to the level of the efferent pseudobranchial foramina anteriorly, because the internal carotids can be traced anteriorly around the side walls of the hypophyseal chamber (see below). By contrast, in neoselachians the efferent pseudobranchial foramen lies in the posterior part of the orbit, and the polar cartilage is comparatively small and makes only a minor contribution to the basicranium (fig. 10; also see discussion).

Ophthalmic and EFFERENT PseudobranCHIAL ARTERIES: Separate foramina for the ophthalmic and efferent pseudobranchial arteries were found by Gross (1937) in Cladodoides. Stensiö (1937) also found separate foramina for these vessels in the Humboldt University braincase. The ophthalmic foramen in both specimens is located ventral and slightly posterior to the optic foramen, adjacent to the articular surface for the palatoquadrate. The efferent pseudobranchial foramen lies even farther ventrally, within the posterolateral margin of the flange forming this articular surface. In CT scans, a passage 

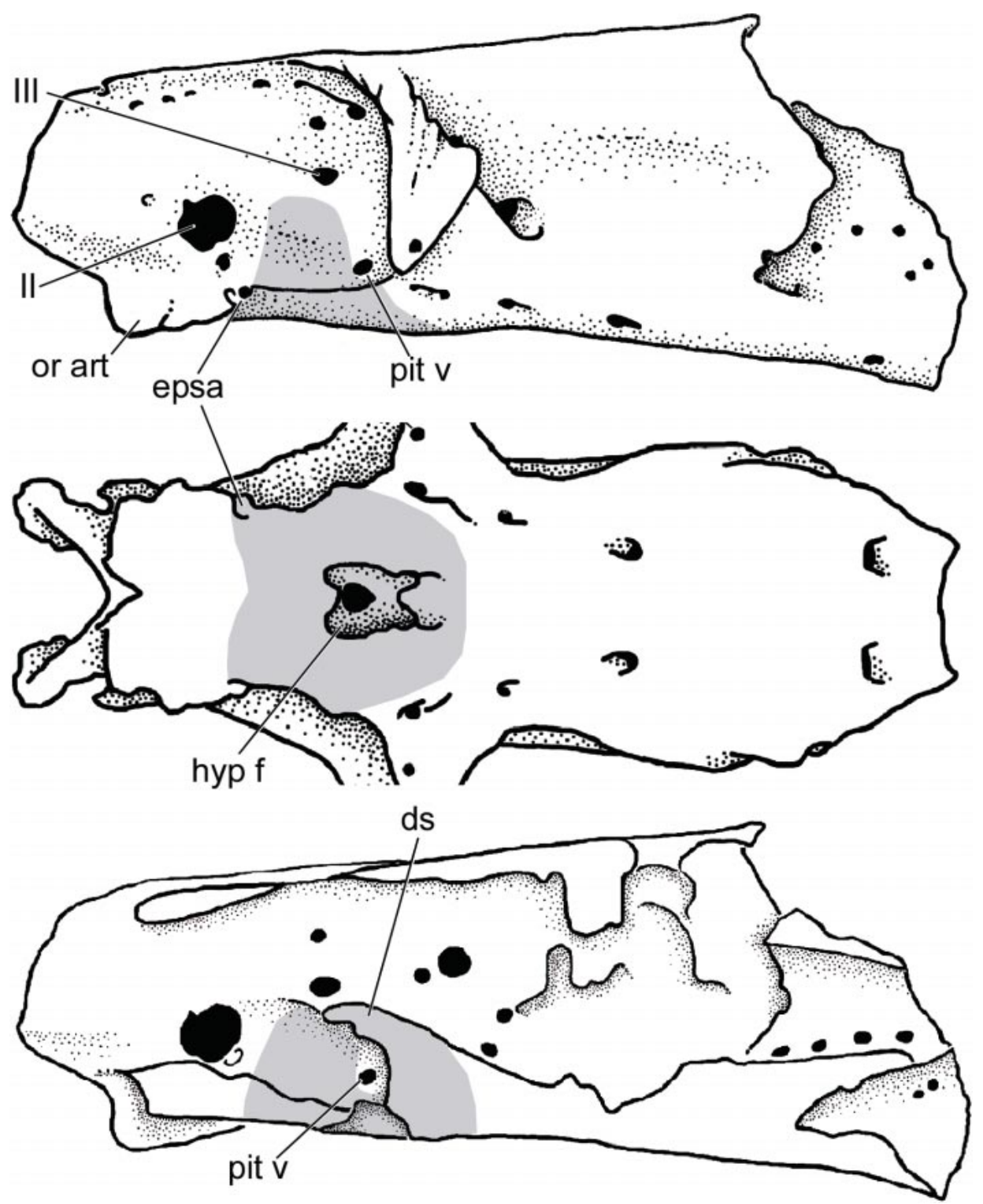

Fig. 9. The Cladodoides braincase in lateral, ventral, and medial views, showing the inferred polar cartilage-derived region (gray area) and important related landmarks. Compare with neoselachians (fig. $10)$.

is seen extending ventrolaterally from the base of the optic canal. This passage branches distally within the thickness of the orbit wall and opens externally at the ophthalmic and efferent pseudobranchial foramina (seen in the endocast and in transverse scan sections through the braincase; figs. 11, 12). In Cladodoides, therefore, the efferent pseudob- ranchial artery entered the cranial cavity laterally and above the trabeculae, as in modern elasmobranchs. A similar arrangement has also been recognized in Orthacanthus and Hybodus (Schaeffer, 1981; Maisey, 1983).

In Orthacanthus, the ophthalmic and efferent pseudobranchial foramina are situated much farther posteriorly than in Cladodoides 


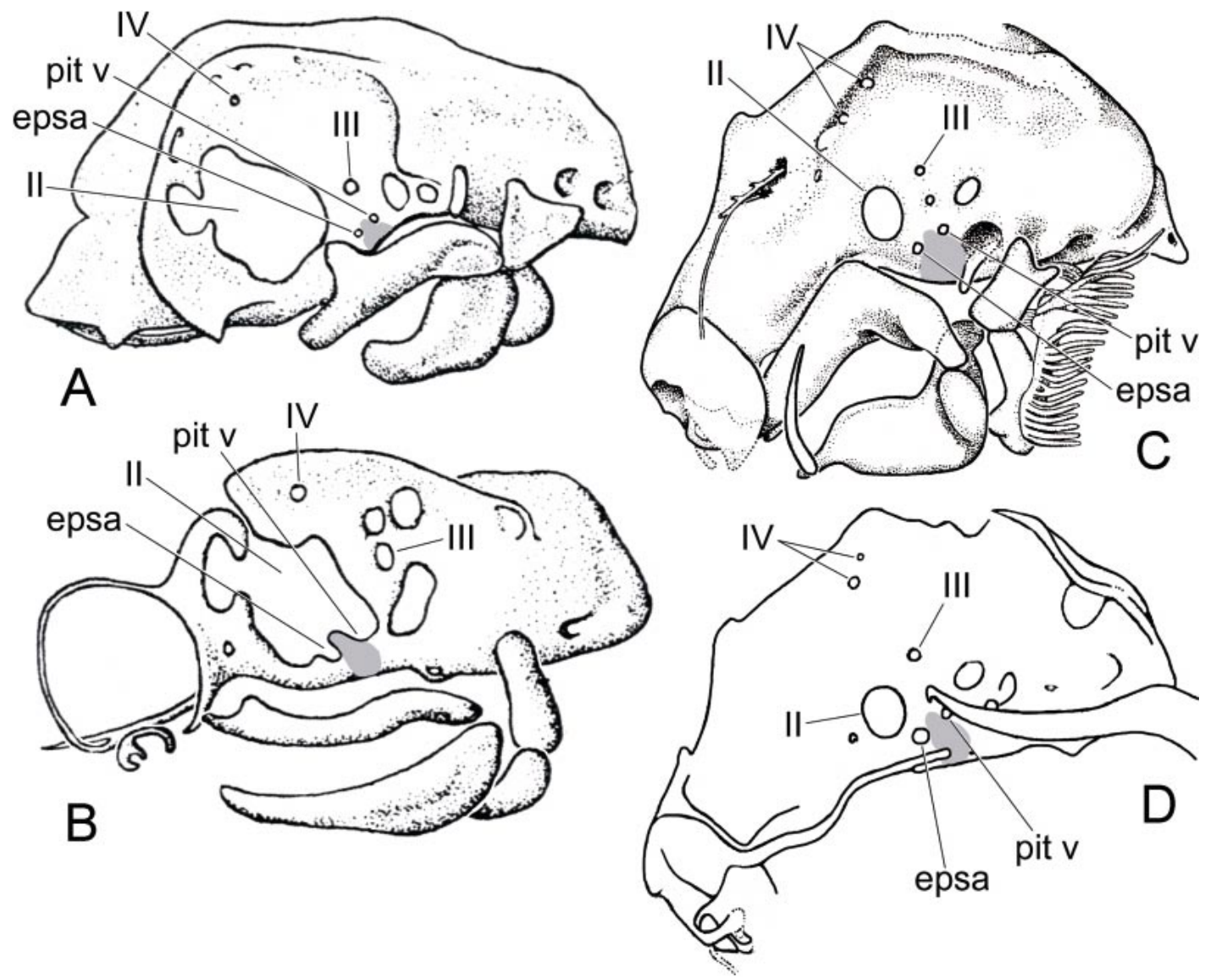

Fig. 10. The extent of the polar cartilage-derived region (gray area) in neoselachians. (A) Squalus 50-mm stage, lateral view; (B) Scyliorhinus 36-mm stage, lateral view; (C, D) Heterodontus 62-mm stage, lateral (C) and medial (D) views. Panels A and B are after De Beer 1931; panels C and D are after Holmgren 1941.

(Schaeffer, 1981: 19). In Tamiobatis vetustus (NMNH 1717) and "Tamiobatis sp." (AMNH 2140), Schaeffer (1981) found only a single foramen and suggested that the efferent pseudobranchial and ophthalmic arteries may have exited together and separated within the orbit. However, the opening he found corresponds topographically to the efferent pseudobranchial foramen in Cladodoides and Orthacanthus. In fact, it cannot be determined if there is only a single foramen in NMNH 1717 because the lower part of the orbital wall (including the expected position of an ophthalmic foramen) is crushed against the suborbital shelf. The "Tamiobatis sp." braincase (AMNH 2140) was cut into thick horizontal slabs before it came to scientific attention, and very few features are visible laterally in the slabbed pieces. It is therefore possible that a separate ophthalmic foramen is present in both fossils, but simply cannot be observed. The position of the efferent pseudobranchial in CMNH 9280 (referred to Tamiobatis vetustus by Williams, 1998) is also uncertain, but it may be represented by a semicircular indentation in the suborbital cartilage slightly anterior to the bucco-hypophyseal fenestra. This would place the artery in a midorbital position, as in Orthacanthus.

Coates and Sequeira (1998) found a small triangular projection in the ethmoid region of Akmonistion, which they termed the posterior ethmoid process. In one specimen, they ob- 


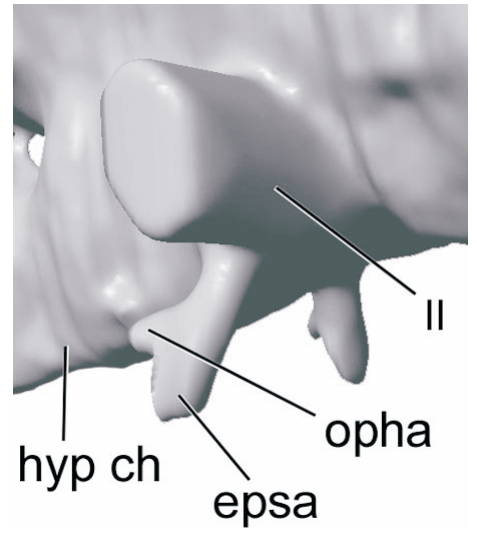

Fig. 11. Cladodoides endocast; detail of passages for optic nerve and ophthalmic and efferent pseudobranchial arteries (surface rendering, oblique anterolateral view).

served a canal in the broken cartilage just anterior to this process, which they interpreted as possibly containing the prehypophyseal branch of the internal carotid artery, or the optic or ophthalmic artery. Comparison with Cladodoides suggests that this canal may indeed have carried the ophthalmic artery. An efferent pseudobranchial foramen has not been identified in Akmonistion; Coates and Sequeira (1998) suggested that the artery entered the cranial cavity farther posteriorly, but alternatively it could have joined the ophthalmic artery within the orbit.

Anterior Palatoquadrate ArticulaTION: In Cladodoides, an articular surface is present on the lateral wall of the internasal plate, extending from below the ethmoid region into the anterior part of the orbit, and meeting the suborbital shelf posteriorly. It consists of a single transverse ridge flanked by wide, slightly concave areas forming a connection with the anterior part of the palatoquadrate (Gross, 1937, 1938) and has been termed either an orbital articulation (e.g., Gross, 1937) or an ethmoidal articulation (e.g., Maisey, 1980; Schaeffer, 1981; Coates and Sequeira, 1998). The efferent pseudobranchial foramen in Cladodoides is located at the boundary of the articular and nonarticular regions of the suborbital cartilage, suggesting that the boundary corresponds to the contact between the embryonic polar and trabecular cartilages in neoselachians (see discussion later in this work).

In Orthacanthus, the articular surface is more complex than in Cladodoides and is scalloped by two or three transverse ridges separated by wide, shallow concavities (Schaeffer, 1981), and there are corresponding ridges and grooves on the medial articular surface of the palatoquadrate (Hotton, 1952). In the slabbed braincase AMNH 2140 (referred to "Tamiobatis sp." by Schaeffer, 1981: figs. 22G, 23G), the articular surface includes at least two ridges and deep grooves. Unfortunately, in the holotype of Tamiobatis vetustus (USNM 1717), the corresponding region is missing, although a suborbital shelf is present. The anterior parts of the palatoquadrates and braincase are missing in the articulated specimen referred to Tamiobatis vetustus by Williams (1998; CMNH 9280), but its suborbital shelf widens appreciably just behind the expected position of an articular surface. An articular process with ridges and grooves is clearly present in isolated palatoquadrates from the Cleveland Shale (e.g., CMNH 9450, probably from a ctenacanth) and in many isolated shark palatoquadrates from the Pennsylvanian black shales of Indiana and Nebraska (Zangerl and Case, 1976; Williams, 1985). In Cobelodus aculeatus and Akmonistion zangerli, there is a prominent flange on the palatine part of the palatoquadrate plus an articular surface on the internasal plate (Zangerl and Case, 1976, Coates and Sequeira, 2001b).

Bucco-Hypophyseal Fenestra and INTERNAL CAROTID ARTERIES: The large size of the bucco-hypophyseal fenestra in Cladodoides may indicate an immature condition (figs. 5, 13), since this part of the basicranium is one of the last to chondrify in extant elasmobranchs (De Beer, 1931). However, the basicapsular fenestra is closed and the parachordal region is completely chondrified, as in adult neoselachians. The precarotid commissure was not mineralized and probably was not chondrified, leaving the internal carotid arteries exposed beneath the braincase before they entered the fenestra. This also suggests immaturity, and the precarotid commissure may therefore have chondrified later in ontogeny, ultimately creating separate canals for the internal carotids. However, 


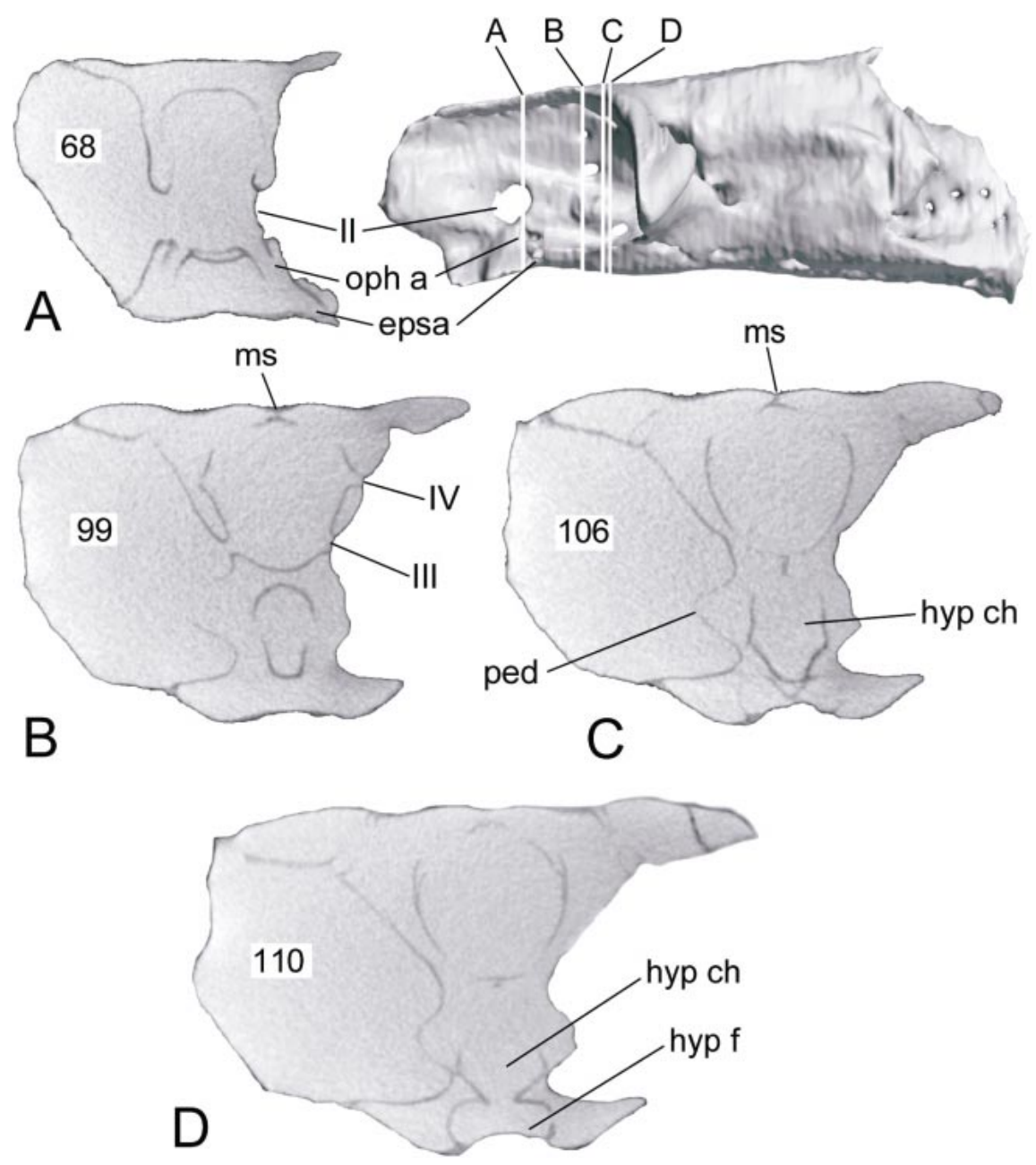

Fig. 12. Transverse CT scan slices of the Cladodoides braincase (levels of slices indicated by white lines on lateral view). (A) Slice 68, through optic foramen; (B) slice 99, through oculomotor and trochlear foramina; (C) slice 106, through pedicel ridge and hypophyseal chamber; (D) slice 110, through hypophyseal chamber and bucco-hypophyseal fenestra.

the bucco-hypophyseal fenestra is small in the Humboldt University braincase (a larger specimen), and separate canals for the internal carotid arteries are present (Stensiö, 1937). The bucco-hypophyseal fenestra in Cladodoides is pinched off from the overlying hypophyseal chamber internally and there is only a single opening for the internal carotids and hypophyseal duct in its roof. The fenestra is probably extracranial and its roof is probably formed from the posterior end of the trabecular-polar cartilage as in neoselachians (the hypophyseal chamber will be described below, in the section on the endocast).

The internal carotid arteries evidently converged gradually but probably did not meet before they entered the braincase, because separate arterial grooves can be traced on each side of the bucco-hypophyseal fenestra. By contrast, in neoselachians these vessels converge almost at a right-angle to the midline, forming part of a "bell-shaped" vascular loop which is confined to the area behind the bucco-hypophyseal fenestra (Schaeffer, 1981). The subsequent course of 


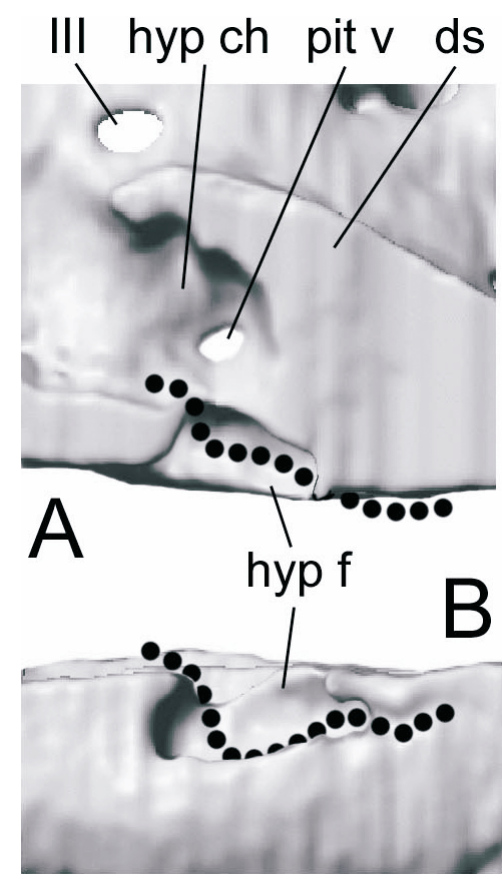

Fig. 13. Detail of the hypophyseal region in Cladodoides. (A) Medial view; (B) ventral view. Anterior to left in both views. The probable course of the internal carotid artery is indicated by a series of dots. After entering the bucco-hypophyseal fenestra (which is essentially an extracranial space), the artery continues around its margin before it enters the braincase via a central opening in the floor of the hypophyseal chamber.

these arteries cannot be traced in Cladodoides, but they presumably joined the orbital, ophthalmic, and efferent pseudobranchial arteries anteriorly and the basilar artery farther posteriorly.

In Tamiobatis vetustus (NMNH 1717), the bucco-hypophyseal fenestra appears to be very large. Originally the fenestra may have been smaller, since the surface of the hypophyseal region has been lost by erosion (Romer, 1964; Schaeffer, 1981). It is also

Fig. 14. "Tamiobatis sp." braincase AMNH 2140 , details of the hypophyseal region in slabbed specimen, showing the relationship of the internal carotid canals and bucco-hypophyseal fenestra. (A) Ventral surface, with internal carotids entering basicranium close together behind bucco-hypo-
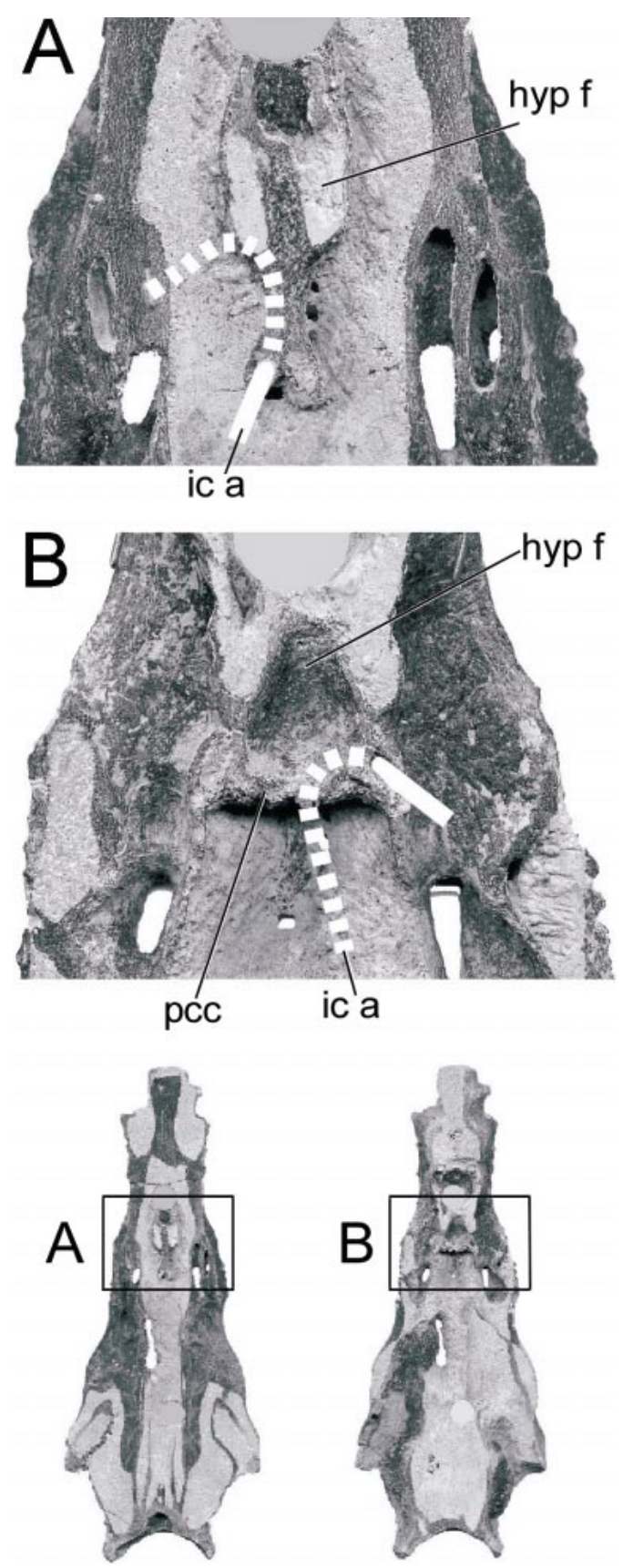

physeal fenestra; (B) internal surface of basicranium, with internal carotids entering cranial cavity separately. The arteries separated from each other and turned laterally within the thickness of the basicranium. They did not extend medially across the bucco-hypophyseal fenestra nor around its lateral walls as in Cladodoides, and they were enclosed by cartilage internally (the precarotid commissure?). 
possible that a precarotid commissure was originally present. The specimen has been restored with its internal carotid canals either completely enclosed (Schaeffer, 1981) or only partly closed (Williams, 1998).

The hypophyseal region is exposed on both sides of the lowermost slab of the "Tamiobatis sp." braincase (AMNH 2140; fig. 14). On the lower surface of this slab, the bucco-hypophyseal fenestra is bisected externally by a narrow area of prismatic calcification which is continuous with the walls of the internal carotid canals. However, on the internal surface of the same slab, the internal carotid canals enter the cranial cavity much farther laterally. Presumably the canals turn sharply outward within the thickness of the basicranial cartilage. Thus, the internal carotids in "Tamiobatis sp." passed obliquely through the basicranium on either side of the bucco-hypophyseal fenestra, as in Cladodoides (and in Tamiobatis vetustus?). The ventral median calcification probably represents part of the floor of the overlying hypophyseal chamber and may have contained a median hypophyseal duct, unlike in Cladodoides, where the duct probably entered the basicranium with the internal carotids. The precarotid commissure in "Tamiobatis sp." was evidently extensive and heavily mineralized. The hypophyseal region is also preserved in one of the fragments referred tentatively to Cladodus elegans. Its internal carotid canals are extensive and converge anteriorly, but the material is too fragmentary to determine the original relationship of the vessels and the bucco-hypophyseal fenestra.

Coates and Sequeira (1998: fig. 5) described paired internal carotid foramina in Akmonistion, separated by a thin median wall or septum similar to that in "Tamiobatis sp." Their observation that the hypophyseal and internal carotid openings were "coincident" in Akmonistion suggests that the precarotid commissure was absent. In Orthacanthus, there is a single median internal carotid foramen separated from the persistent buccohypophyseal fenestra by a broad precarotid commissure. Schaeffer (1981) reconstructed its bucco-hypophyseal fenestra with a deep, tapering canal connected to the hypophyseal chamber, although this region was not well preserved in the material available. In Cla- doselache, the internal carotids probably lay in open grooves in the basicranium, not within enclosed canals (Williams, 1998; Coates and Sequeira, 1998; cf. Schaeffer, 1981). Cobelodus aculeatus has been restored with internal carotids in canals (Zangerl and Case, 1976; Schaeffer, 1981; Coates and Sequeira, 1998), but the better preserved "Cobelodus" braincase casts doubt on these earlier reconstructions, and the internal carotid arteries of symmoriid sharks probably did not follow a conventional arrangement (Maisey, 2004a). In fact, there is little evidence that internal carotids were enclosed completely by basicranial canals in any fossil or extant elasmobranch.

In neoselachians, the internal carotids may enter the braincase either separately or together, but no clear phylogenetic pattern has emerged (Holmgren, 1941: char. 9). It is therefore doubtful whether much phylogenetic significance can be attached to the presence of single or paired internal carotid foramina in extinct sharks (cf. Coates and Sequeira,1998: char. 6), although paired foramina are certainly more usual in Paleozoic taxa. A single carotid foramen is present in Tristychius and Hybodus (Dick, 1978; Maisey, 1983). Internal carotids are absent in chimaeroids, and their brain receives its blood supply entirely from the efferent "pseudobranchial" artery (De Beer and Moy-Thomas, 1935).

Optic Pedicel, Optic Nerve, AND OcuLOMOTOR NERVE: In Cladodoides, the orbital wall contains a large optic foramen centrally (figs. 1, 2, 6), which probably contained the optic (retinal) artery as well as the optic nerve (as in Chlamydoselachus; Allis, 1923a). Immediately behind this foramen is an extensive longitudinal ridge, which Gross (1937) interpreted as the basal attachment area for the optic pedicel. That interpretation is supported by the topographic position of the ridge, the absence of calcification distally (where the pedicel would attach in life), and the fact that the internal surface of the cartilage beneath the ridge is continuously calcified, with no foramina for nerves or blood vessels passing through it. Gross (1937) depicted the apical part of the pedicel ridge as rough and largely uncalcified, but CT scan slices of the unprepared right orbit show that 

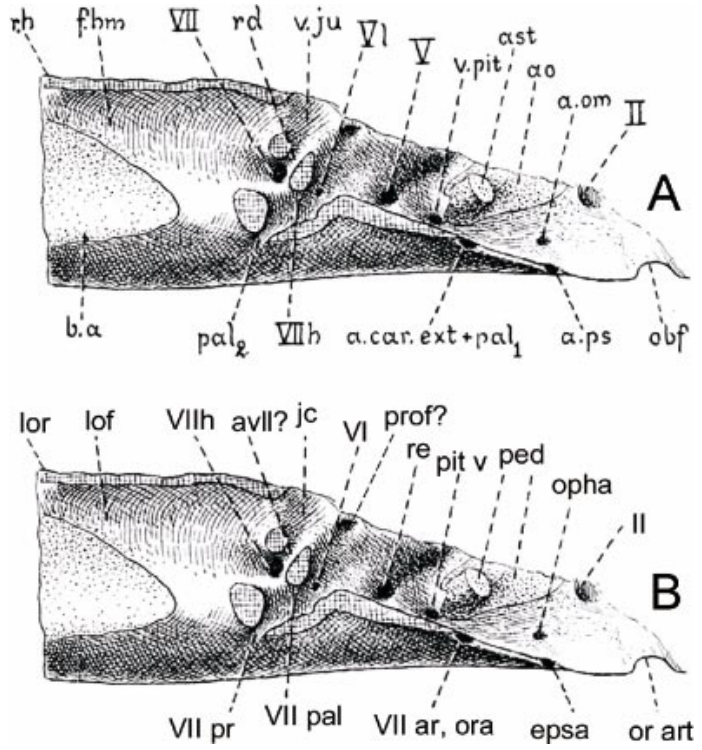

Fig. 15. Ventral region of the orbit in the Humboldt University specimen ("Cladodus hassiacus"). (A) From Stensiö 1937; (B) with certain features reidentified following the present interpretation of Cladodoides. The unlabeled feature in panel B is simply the clipped wall of the otic capsule. Differing interpretations include the external rectus insertion (previously identified as the trigeminal foramen), the inferred position of the anteroventral lateral line nerve, and reinterpretation of the supposed hyomandibular facet as part of the lateral otic fossa.

the ridge is extensively calcified except at its very tip, suggesting that the left ridge was damaged during preparation. A similar arrangement was described by Stensiö (1937: fig. 7) in the Humboldt University braincase. He showed both an "eye stalk area" and an actual "eye stalk process" corresponding to the longitudinal ridge in the Senckenberg specimen (figs. 15, 16).

Transverse CT scan sections show that the pedicel ridge in Cladodoides is positioned lateral to the hypophyseal chamber (fig. 12), unlike in modern sharks where the pedicel attachment area is typically located above the level of the chamber (e.g., Chlamydoselachus; Allis, 1923a; Echinorhinus, Centroscyllium, Squaliolus; Shirai, 1992). According to Schaeffer's (1981) reconstruction of Orthacanthus, the pedicel seems to be positioned above the hypophyseal chamber, but this is not clear from the specimens he illustrated. In the slabbed "Tamiobatis sp." braincase (AMNH 2140), the presumed pedicel attachment site lies in the same horizontal plane as the hypophyseal chamber, as in Cladodoides (Schaeffer, 1981: fig. 23F). In Cladodus elegans, the orbital wall is inflated in the expected position of the optic pedicel behind a large foramen (probably for the optic nerve), but other features typically accompanying the pedicel have not been found.

In neoselachians, the base of the optic pedicel lies just behind and below the oculomotor foramen. Judging from the position of the foramen in Cladodoides, the anterior part of this ridge probably did not actually support the pedicel, which probably arose farther posteriorly near the abducent foramen and the presumed origin of the posterior rectus muscle (see below). An optic pedicel is present in Pucapampella but is unknown in Gladbachus or Doliodus.

In Cladodoides, the oculomotor foramen lies just above the middle of the pedicel ridge fig. 6). The oculomotor nerve probably followed the same course as in neoselachians, leaving the brainstem near the midline of the mesencephalon, then entering the orbit and dividing into a dorsal branch for the superior and anterior rectus eye muscles, and a ventral branch which subdivides into branches for the inferior oblique and inferior erectus eye muscles (e.g., Notorynchus; Daniel, 1934). This area is missing in the Humboldt University braincase, and Stensiö (1937) did not identify the oculomotor foramen, which presumably lay farther dorsally.

TROCHLEAR Nerve: In neoselachians, the trochlear nerve arises in the mesencephalon, and then its roots cross and the nerve leaves the roof of the brain between the mesencephalon and cerebellum, entering the orbit to supply the superior oblique eye muscle.

The trochlear foramen in Cladodoides lies almost directly above the oculomotor foramen, and the CT scan shows that both nerve openings lead directly into the cranial cavity. The region of the orbit including the trochlear foramen is probably missing in the Humboldt University braincase. Schaeffer (1981) identified the trochlear foramen of Orthacanthus in an unusually anterior position (in front of the optic foramen), but it is located 

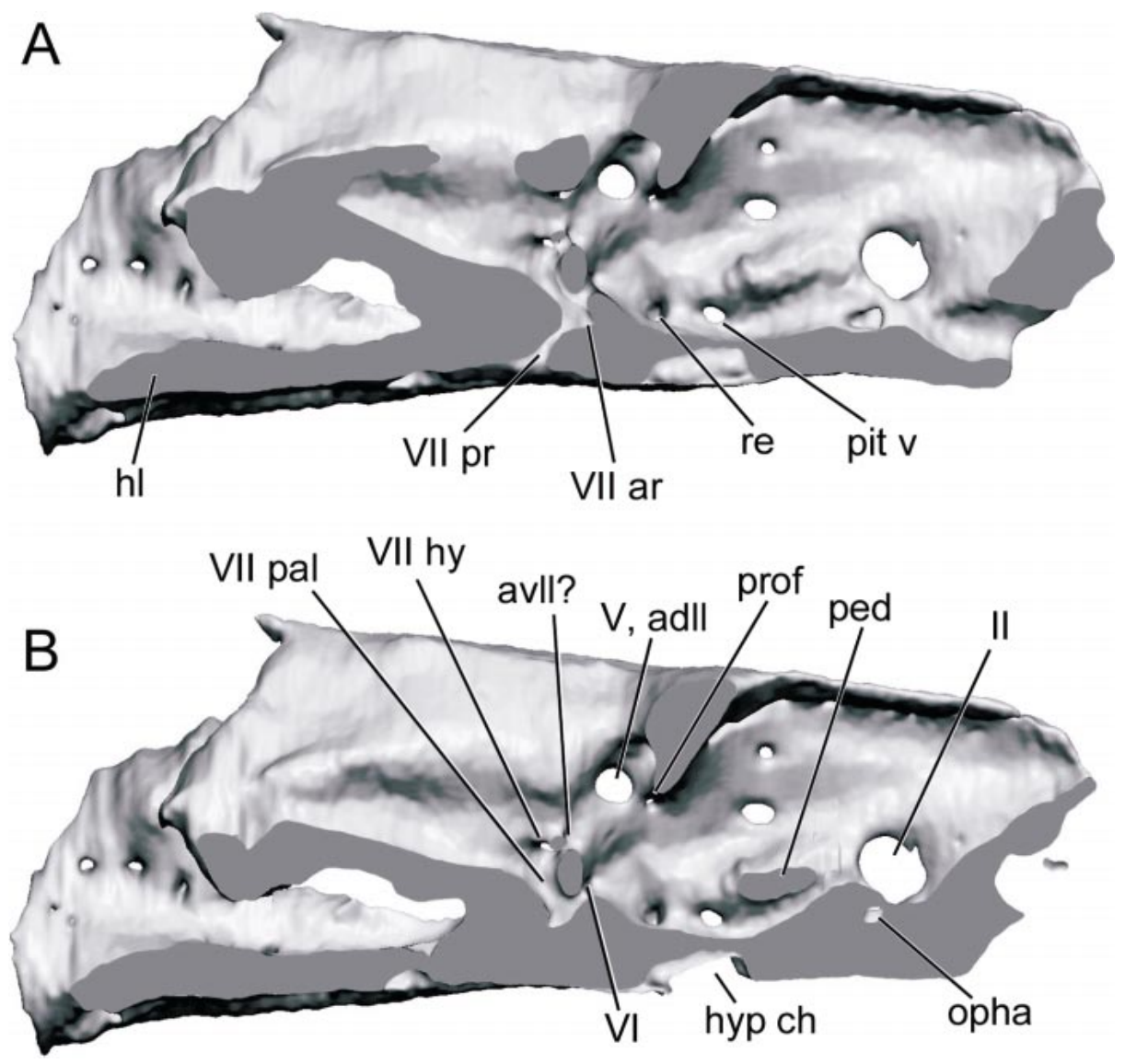

Fig. 16. Surface rendering of the scanned Cladodoides braincase with clipping planes cutting through the postorbital process and suborbital shelf. Both views are reversed to facilitate comparison with figure 15. The clipping plane in panel $\mathbf{A}$ is almost parallel to the sagittal plane. In panel $\mathbf{B}$ the plane is more oblique, cutting the posterior part of the braincase farther laterally and the anterior part farther medially. Orientation data for clipping planes are as follows: Plane (axis, position, rotation); A (X, 14.08, 77.0; Y, 5.0, 109.0; Z, 11.34, 270.0); B (X, 10.56, 77.0, Y, 1.60, 121.0, Z, 0.80, 270.0). These values represent positions along and angular deviations from the surface rendering's original XYZ axes.

farther posteriorly (i.e., in a more conventional position) in other Orthacanthus material (Maisey, 1983: fig. 14B). The most likely explanation of this discrepancy is that Schaeffer's (1981) supposed trochlear foramen is actually the anterior profundal foramen. Hybodus basanus has been restored with separate trochlear and profundal foramina, both situated anteriorly in the orbit (Maisey, 1983: fig. 13A).

ABDUCENT NERVE: Gross (1937) considered that the abducent nerve was separate in Cladodoides and he identified its foramen within the jugular canal. However, such a position would place the nerve rather high in the orbit, whereas the abducent nerve typically arises caudoventrally below the trigeminal and facial ganglia. Instead, the abducent nerve (assuming it had a separate foramen in Cladodoides) may have exited much lower in the cranial wall via a small foramen ventral to the main trigeminal foramen. Gross (1937) considered this foramen to house an unspecified branch of the facial nerve, but its passage has no obvious internal connection with the main facial foramen (see below). Furthermore, the foramen lies at the same level in the orbit as the optic pedicel (although still some distance behind it), approximately where the abducent nerve leaves the 


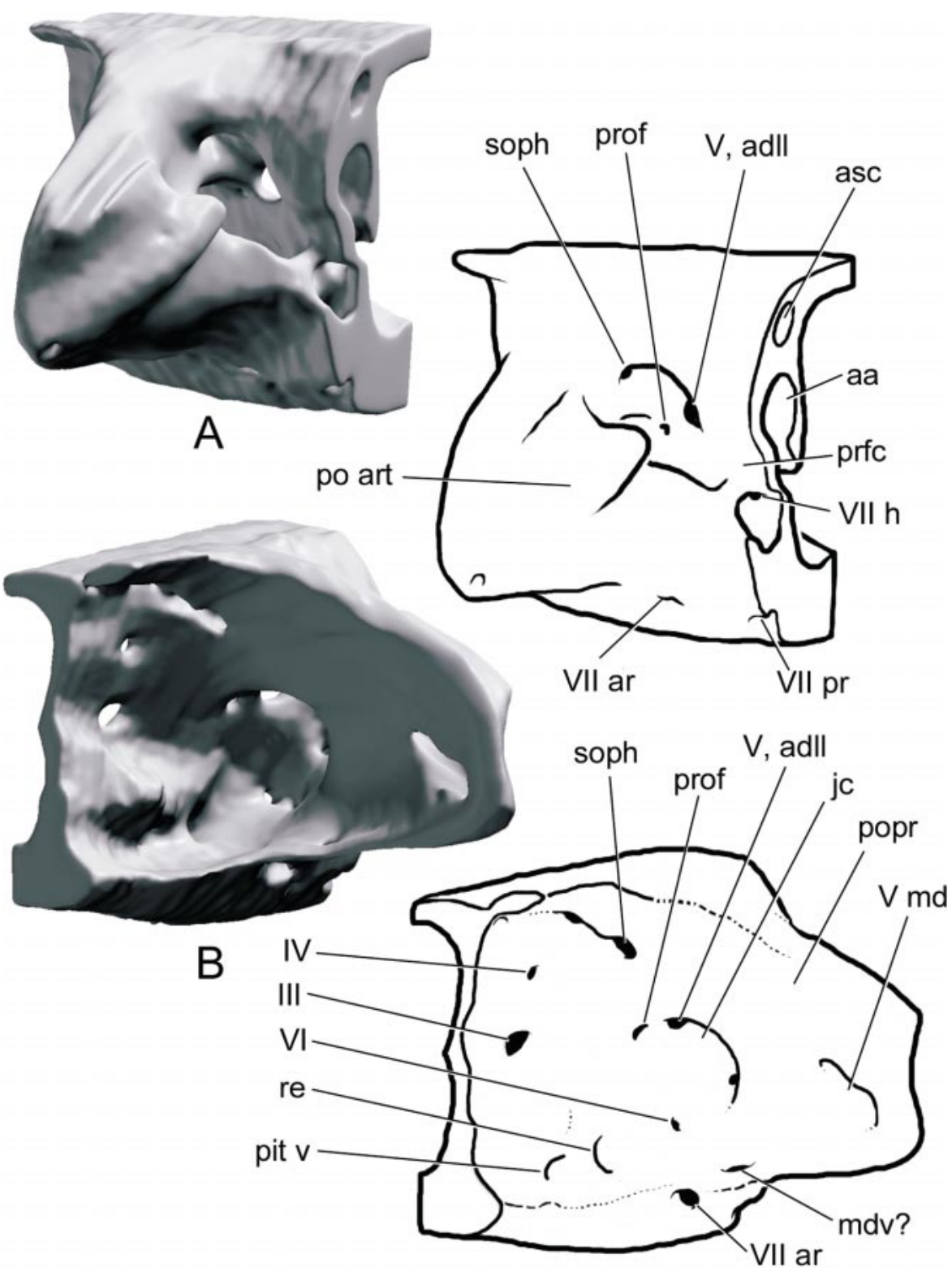

Fig. 17. Postorbital region of Cladodoides braincase (surface rendering), oblique perspective detail views. (A) Posterolateral view; (B) anterolateral view; (C) anteromedial view; (D) posteromedial view. Differences from Gross (1937) involve the positions of the abducent and profundal nerve. The dorsum sellae overhangs the deep hypophyseal chamber, and the lateral hypophyseal ridge delimits the side wall of this chamber. The superficial ophthalmic ramus passes through the postorbital process and emerges posteriorly just above the main trigeminal and facial nerve foramina.

braincase in neoselachians. The supposed abducent foramen identified by Gross (1937) may instead have housed the profundal nerve (see next section). The foramen regarded here as housing the abducent nerve is ob- scured by the postorbital process in lateral view (fig. 6), but it can be seen in the oblique views of the orbit and postorbital process (fig. 17B, D).

Several foramina are present in the ventral 


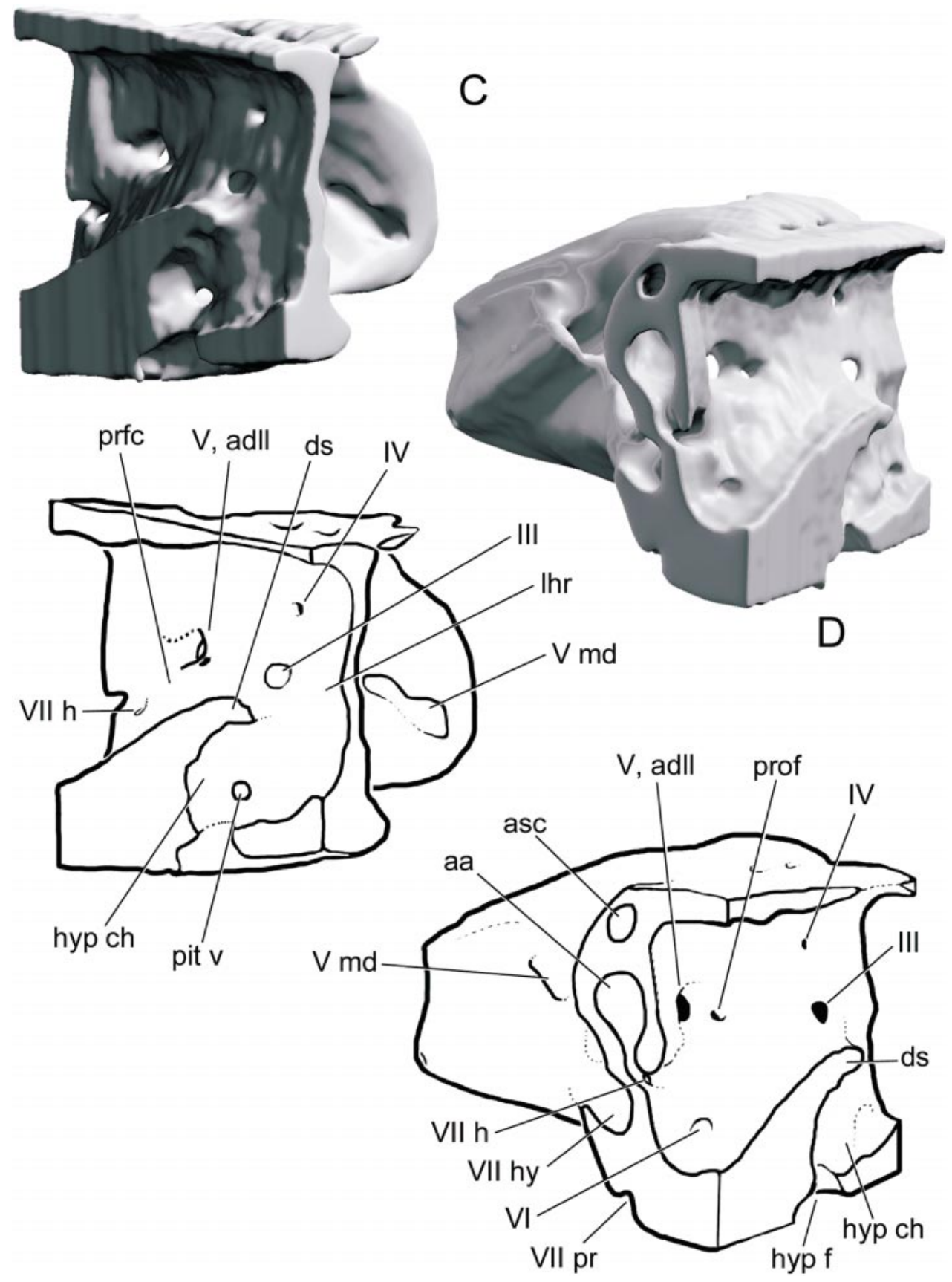

Fig. 17. Continued.

part of the orbit in the Humboldt University "Cladodus" hiassicus braincase (fig. 15) and their arrangement agrees closely with the Senckenberg specimen (the corresponding area in this specimen is shown in fig. 16).
The foramen said to house an unspecified facial ramus in Cladodoides by Gross (1937) apparently corresponds to Stensiö's (1937) foramen "for the roots of the lateralis fibres to the trigeminus branches" (Vl, fig. 15A) in 
"C." hiassicus. However, the corresponding foramen in Cladodoides probably contained the abducent nerve, and this is also possible in the case of "C." hiassicus (fig. 11B).

The position of the abducent foramen has not been determined in Tamiobatis vetustus or "Tamiobatis sp.", but in Orthacanthus it is located next to a large trigemino-pituitary fossa high up in the orbit, within the opening of the jugular canal (Maisey, 1983: fig. 14). In Hybodus basanus, the abducent foramen is lower in the orbit, sandwiched between the trigemino-pituitary fossa and the exit for the hyomandibular trunk of the facial nerve.

In gnathostomes, the abducent nerve arises from a nucleus in the medulla and passes anterolaterally into the orbit, where it supplies the external (= posterior) rectus eye muscle. In neoselachians, a separate abducent foramen is present in Oxynotus and Centrophorus but not in Etmopterus (Holmgren, 1941). In Chlamydoselachus, the abducent and trigeminal nerves exit together in the upper part of the trigemino-pituitary fossa (Allis, 1923a), but in hexanchiforms the abducent nerve has its own foramen. The abducent and trigeminal nerves also share a foramen in Heterodontus, Torpedo, and in the chimaeroid Hydrolagus (Gardiner, 1984a). Gardiner (1984a: 248) concluded that in primitive gnathostomes the abducent nerve left the cranial cavity below and behind the dorsum sellae, and that in osteichthyans the dorsum sellae was primitively ossified by the basisphenoid. The abducent foramen lies below and behind the dorsum sellae in Cladodoides fig. 7), and in chimaeroids and some neoselachians (e.g., Squalus), and this may represent the primitive pattern for chondrichthyans. However, in some modern sharks the abducent foramen may be located above and behind the dorsum sellae, which may represent an autapomorphic condition (e.g., Heterodontus).

Profundal Nerve: Ever since Wijhe (1882) recognized that the profundal nerve in Scyliorhinus was distinct, there has been a tendency to regard the profundal $\left(\mathrm{V}_{1}\right)$ and trigeminal $\left(\mathrm{V}_{2}+\mathrm{V}_{3}\right)$ nerves as separate, culminating in the work of Northcutt and Bemis (1993). As stated above, the profundal nerve in Cladodoides may have lain within the supposed abducent foramen of Gross (1937), al- though this is admittedly an unsatisfactory interpretation because the profundal nerve in neoselachians generally does not leave the braincase separately (the condition in osteichthyans is more variable; Gardiner, 1984a: 238). The foramen in question is located behind the postorbital process, in the ventral part of a deep depression ("Gangliongrube" of Gross, 1937) which also contains the trigeminal foramen (fig. 17). Gross (1937) suggested that this depression housed extracranial components of the trigeminal and anterodorsal lateral line ganglia. If that is correct, the profundal ganglion presumably lay farther ventrally, perhaps contacting the anterodorsal lateral line ganglion dorsally. Conceivably, the ganglia of both the facial's superficial ophthalmic ramus and profundal nerve in Cladodoides were closely associated with each other, as in Mustelus (Norris and Hughes, 1920: fig. 23). Both ganglia may have occupied the supposed profundal foramen, although such an arrangement would separate them from the main trigeminal and anterodorsal lateral line trunks. Since all these nerves typically occupy the prootic foramen in neoselachians, this interpretation presumes that the embryonic prootic foramen was partly closed in Cladodoides, leaving the two small openings observed.

In neoselachians, the profundal ganglion is usually distinct but its position is variable; in Squalus it is completely extracranial and makes contact dorsally with the anterodorsal lateral line ganglion, but in Mustelus it is intracranial (Norris and Hughes, 1920). In Chlamydoselachus, the profundal nerve issues through the trigeminal foramen (Allis, 1923a), not separately as stated by Gardiner (1984a: 240; this condition also occurs in many other neoselachians). The profundal nerve usually passes deep within the orbit in neoselachians, initially passing between the superior and inferior rectus muscles, then beneath the anterior rectus and between the oblique muscles (Daniel, 1934); the nerve may have followed a similar course in Cladodoides.

Trigeminal Nerve: Gross (1937) associated only one foramen with the trigeminal nerve in Cladodoides, within a deep depression just behind the postorbital process and a considerable distance from the pituitary fo- 
ramen and from the pit for the external rectus muscle (both of which lie within the orbit; fig. 17). Schaeffer (1981: 45) found such a trigeminal arrangement "improbable" and argued instead that the trunks of the trigeminal and facial nerves passed through the foramen "RVII" of Gross (1937). In fact, scanning provides considerable support for Gross' (1937) original observations. Moreover, Schaeffer's (1981) proposals are incompatible with the present interpretation of "RVII" as the abducent foramen. The main trunk of the trigeminal nerve apparently exited the braincase exactly where Gross (1937) suggested, probably accompanied by the trunk of the anterodorsal lateral line nerve. Since the trigeminal nerve emerges in front of the postorbital wall in neoselachians, holocephalans, and osteichthyans, its more posterior position in Cladodoides is unusual (the mandibular ramus probably entered the postorbital process; see below and figs. 18B, 19). Nevertheless, in Acanthodes the mandibular ramus was apparently located even farther posteriorly than in Cladodoides (behind the postorbital process; Miles, 1968, 1973; Jarvik, 1977).

The supposed trigeminal foramen in the braincase of "Cladodus" hiassicus (Stensiö, 1937) corresponds closely with the pit for the external rectus muscle in Cladodoides, which raises three alternative possibilities. Either the trigeminal foramen is not preserved in " $C$." hiassicus and lay instead farther dorsally (the preferred interpretation, since it agrees with the arrangement in Cladoidoides; compare figs. 15, 16); or the trigeminal and facial nerves may have shared a single foramen in " $C$.' hiassicus (a very different arrangement from Cladodoides and elasmobranchs generally); or the supposed facial foramen in " $C$." hiassicus might be for the trigeminal nerve (in which case the facial foramen presumably lay in a different position than in Cladodoides, and has simply not been found).

The middle cerebral vein in Cladodoides may have accompanied the trigeminal nerve as in neoselachians (Gardiner, 1984a), although there is no direct evidence of its position.

Facial Nerve: Excluding the lateralis components, Gross (1937) identified four fo- ramina for branches of the facial nerve ("V + VII", "VII h", "RVII", and "rd"). However, his "foramen V + VII" represents the dorsal part of the jugular canal (see below) and probably did not contain the facial nerve proper, while his foramen "RVII" may be the abducent foramen (see above). However, his foramen "VII h" (located below and slightly behind the cartilage forming the floor of the jugular canal in the postorbital process; fig. 2) almost certainly contained the main facial (= hyomandibular) trunk (fig. 17). Adjacent to this foramen is another smaller opening, which Gross (1937) identified as housing either a ramule of the facial nerve or an unspecified blood vessel. Scanning shows that this opening is confluent internally with the main hyomandibular canal and that the two are merely separated superficially by a short cartilaginous bridge extending between the postorbital process and otic capsule immediately below the prefacial commissure. The lowermost of the two external openings defined by this bridge probably contained the hyoidean ramus, while the upper one probably housed the laterally directed mandibular ramus. The anteroventral lateral line nerve in Cladodoides probably left the braincase along with the hyomandibular trunk as in neoselachians, passing through a short passage immediately after leaving the braincase (fig. 16).

Scanning reveals that the "canal" which Schaeffer (1981) thought contained the orbital artery does not in fact pass directly into the orbit as one might expect; instead, it runs dorsally toward the hyomandibular foramen and then turns abruptly downward to enter the orbit at the base of the postorbital process (fig. 17). This "canal" therefore consists of two passages, one anterior to the other, which converge dorsally and probably contained the anterior and posterior ramules of the palatine ramus (as proposed by Gross, 1937). The arrangement closely resembles that seen in Squalus, where the palatine ramus passes through the floor of the lateral commissure ventrally (as in many squaloids; Norris and Hughes, 1920; Holmgren, 1941). However, the palatine ramus in Squalus divides into anterior and posterior ramules below the lateral commissure, whereas in Cladodoides this division occurs within the equivalent cartilage. 


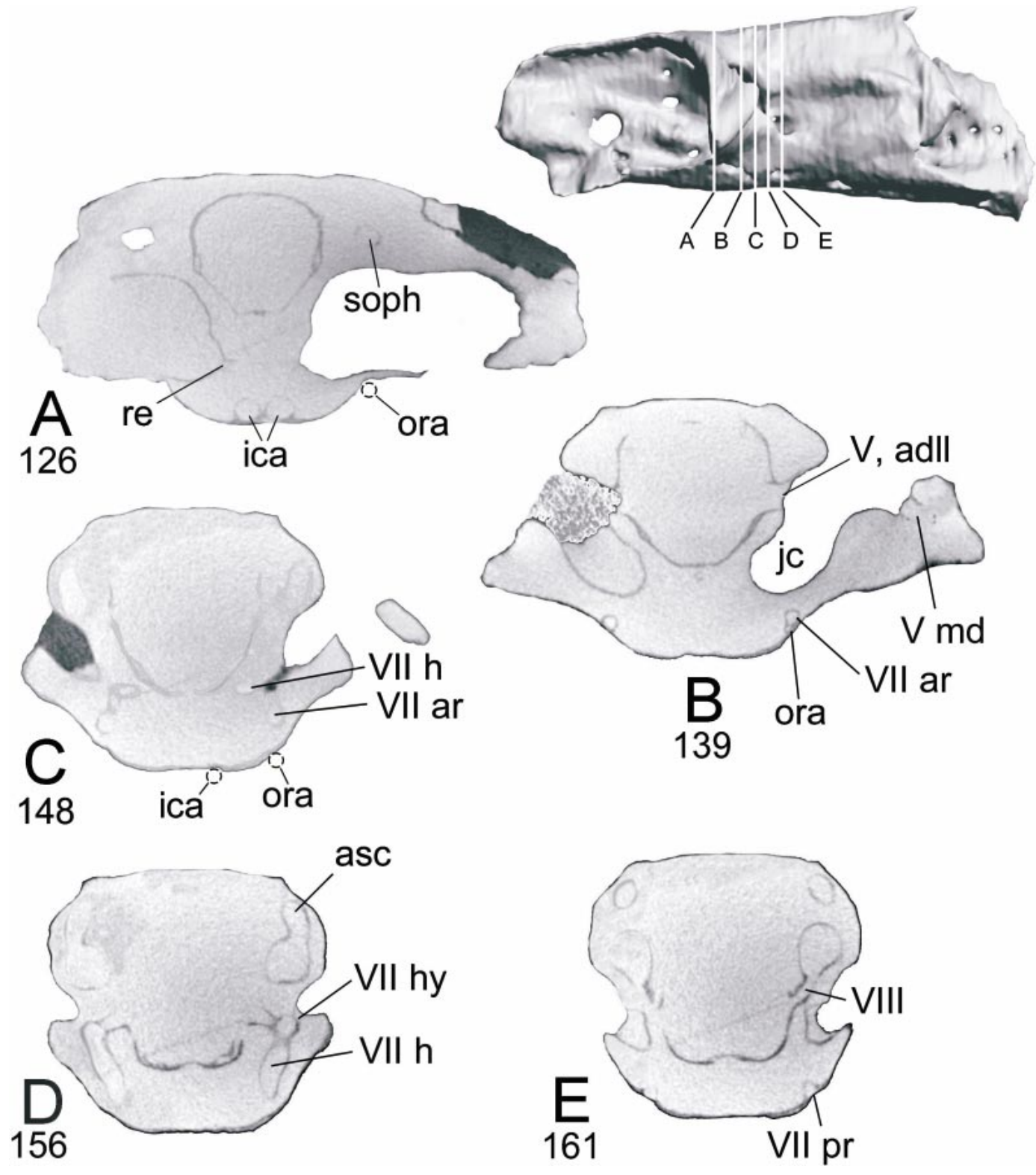

Fig. 18. Transverse CT scan slices of the Cladodoides braincase (levels of slices indicated by white lines on lateral view). (A) Slice 126, through the postorbital process and the presumed origin of rectus muscle; (B) slice 139, through the jugular canal, showing the combined exit for the trigeminal and anterodorsal lateral line nerves, and the convergence of the anterior passage for the palatine ramus with the groove for the orbital artery; (C) slice 148, showing the separation of passages for the hyomandibular trunk and posterior branch of the palatine ramus; (D) slice 156, showing the exit of the hyomandibular trunk; (E) slice 161, showing the passage of the octaval nerve entering the otic capsule 

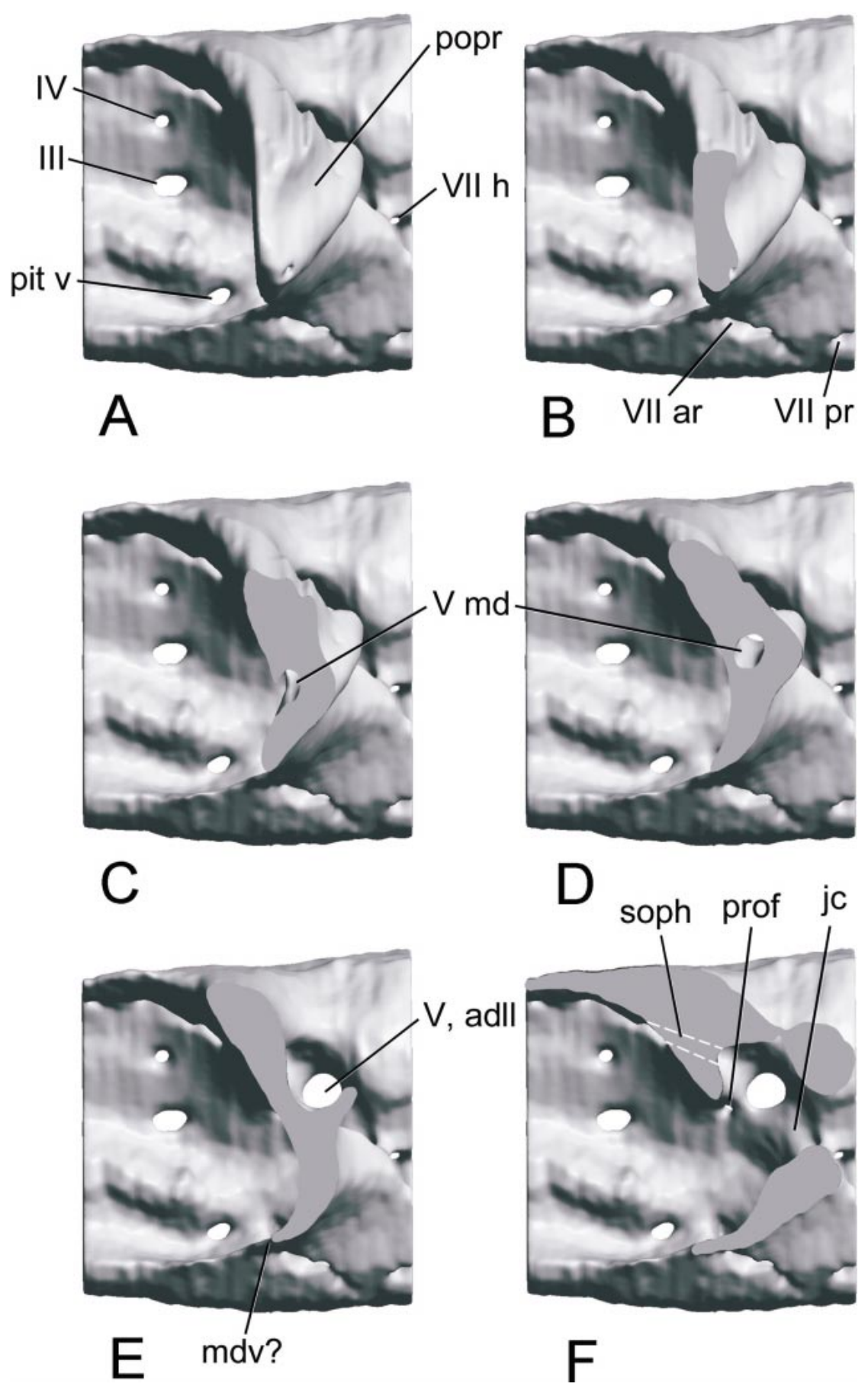

Fig. 19. A series of clipping plane sections through the surface rendering of the left postorbital process in Cladodoides. (A) Lateral view, showing foramina for oculomotor, trochlear, and facial nerves and pituitary vein; (B) showing foramina for the anterior and posterior ramules of palatine ramus; $(\mathbf{C}$, D) canal for mandibular ramus of trigeminal nerve (or main buccal-maxillary complex?); (E) showing alignment of trigeminal foramen with canal for mandibular ramus in postorbital process; (F) slice through jugular canal, with position of superficial ophthalmic canal indicated by white dashed lines. This section just clips the anterior ampulla (inflated area behind trigeminal foramen). 
In Squatina, the lateral commissure is more extensively chondrified than in Squalus, but the palatine ramus arises behind the process and does not pass through cartilage.

In Cladodoides, the passage for the anterior ramule of the palatine ramus merges with a groove for the orbital artery just behind the orbit. This opening apparently corresponds to "foramen one" of Williams (1998), while the posterior palatine foramen corresponds to his "foramen two". Since previous interpretations of the palatine foramina in fossil sharks are inextricably interwoven with scenarios about the basicranial arteries, further discussion of the palatine nerve will be found in the section on the arterial circuit (see below). There is little doubt that the palatine ramus in Cladodoides continued anteriorly to pass underneath the orbital articulation as in neoselachians.

A virtually identical arrangement is seen in Stensiö's (1937) lateral view of the " $\mathrm{Cla}$ dodus" hiassicus braincase (fig. 15A). Its main facial foramen is surrounded by three narrow cartilaginous projections forming passages for the palatine rami and the main hyomandibular trunk. However, the latter probably did not pass where Stensiö (1937) indicated (through a "rostro-caudal facialis canal"). Instead, the hyomandibular trunk was probably directed posteriorly and the "facialis canal" probably contained the anterior ramule of the palatine ramus and the abducent nerve (fig. 15B). The passage for the posterior palatine ramule agrees in the Senckenberg and Humboldt University specimens.

The course of the facial nerve is known only in a few extinct sharks besides Cladodoides. In the primitive neoselachian Synechodus there is a large foramen in the back of the orbit, possibly for the hyomandibular trunk (Maisey, 1985). In Hybodus basanus, there is also a large posteriorly directed opening for the hyomandibular trunk within the jugular canal of the postorbital process (Maisey, 1983), and the palatine ramus may have passed through a large opening in the chondrified floor of the jugular canal (previously identified as a foramen for the efferent hyoidean artery). According to Schaeffer (1981), in Orthacanthus and "Tamiobatis sp." (AMNH 2140) the hyomandibular fo- ramen lies behind the postorbital process. In Tamiobatis vetustus (NMNH 1717) there is a foramen in the lateral wall of the otic region slightly behind the postorbital process. According to Romer (1964) this contained the middle cerebral vein, but comparison with Cladodoides, Orthacanthus, and "Tamiobatis sp." suggests that it housed the hyomandibular trunk of the facial nerve. Romer (1964) considered that the facial foramen lay higher on the lateral wall of the braincase immediately behind the dorsal part of the postorbital process, but comparison with Cladodoides suggests it contained the trigeminal and anterodorsal lateral line nerves. The position of the facial foramen is unknown in Cladodus elegans.

OCTAVOlATERAL NeRVES: The anterodorsal lateral line ganglion was probably extracranial in Cladodoides, but it is not possible to tell whether its superficial ophthalmic and buccal portions were confluent (as in Squalus) or widely separated (as in Mustelus).

Gross (1937) found that the superficial ophthalmic ramus in Cladodoides entered the orbit via a foramen in the roof of the postorbital process before continuing anteriorly in a shallow groove in the ventral surface of the supraorbital cartilage. Here, it gave off numerous dorsal ramules that pierced the cartilage to supply the supraorbital sensory canal. Scanning shows that the canal for this nerve passes completely through the postorbital process above the jugular canal and emerges posteriorly just lateral to the presumed exit of the trigeminal nerve (figs. 1619). However, the superficial ophthalmic ramus in "Tamiobatis sp." (AMNH 2140) probably passed through the jugular canal just below its roof, instead of through a separate canal (this was indicated as a shaded area within the jugular canal by Schaeffer, 1981: fig. 23D). In both Cladodoides and "Tamiobatis sp.", the trigeminal and anterodorsal lateral line nerves probably left the braincase together, and the anterodorsal lateral line nerve subsequently divided into superficial ophthalmic and buccal rami behind the postorbital process. The superficial ophthalmic ramus then passed through ("Tamiobatis sp.") or above (Cladodoides) the jugular canal, probably accompanied by the trigeminal superficial ophthalmic ramus. 
Gross (1937) also suggested that the buccal ramus in Cladodoides entered the postorbital process proximally (where it supposedly gave off dorsal ramules for part of the infraorbital sensory canal), although he did not describe the course of the ramus in detail. The present investigation supports his interpretation in part, but the internal morphology of the postorbital process is more complex than he supposed (see below). The course of the buccal ramus has not been determined in the Humboldt University specimen.

In both Cladodoides and "Cladodus" hiassicus, the anteroventral lateral line nerve may have passed through a small canal immediately anterior to the facial foramen in the floor of the jugular canal figs. 15, 16). However, in " C." hiassicus, Stensiö (1937) simply identified this canal as connecting the jugular and facial nerve canals (fig. 15). Nevertheless, it is remarkably similar to a small canal thought to be for the anteroventral lateral line nerve in Cladodoides.

The octaval (acousticovestibular) nerve is considered separately in the section on the otic region (see below).

POSTERIOR PART OF ORBIT: According to Schaeffer (1981: 19), the external rectus musculature in Paleozoic sharks supposedly originated in a large fossa, "in part the trigemino-pituitary fossa of Allis (1923)'. However, the structure he identified as a trigemino-facial recess in Cladodoides is in reality little more than a depression in the postorbital wall that may have housed the lateral head vein or posterior rectus muscles (fig. 20A), but certainly not the trigeminal or facial ganglia, which were excluded from the orbit by the postorbital process, as Gross (1937) originally recognized (figs. 2, 6, 8A, $17,19)$. While it is true that the trigeminal foramen in Cladodoides lies within a small recess, this is located behind the postorbital process and is completely separated from the pituitary foramen, rectus muscle insertion, and abducent foramen (all of which are located below the ridge forming the optic pedicel; fig. 17B). A deep, narrow, matrix-filled pit is present in the back of each orbit. Although these pits are not readily observed on the specimen, they can be seen in CT scan sections and oblique surface renderings of the orbit (re, figs. 17, 18). They probably contained the origin of the external rectus muscle and are equidistant from the pituitary foramen anteriorly and the abducent foramen posteriorly, but they are remote from the trigeminal and facial foramina. Gardiner (1984a) proposed that, in gnathostomes, the external rectus muscles primitively originated posterior to the optic nerve on the interorbital septum. Their inferred arrangement in Cladodoides agrees with this primitive arrangement and there is certainly no evidence of backward growth of the external rectus muscles to form a posterior myodome as in actinopterygians. When internal and external views of the braincase are compared, the rectus muscle pit and the dorsum sellae are seen to be aligned with each other on the lateral and medial surfaces of the cartilage respectively (e.g., figs. 6, 17). The pituitary foramen, abducent foramen, and the pit for the external rectus muscle are all located in the floor of the orbit at the anterior end of the jugular canal. Thus, all these structures are separated from the trigeminal and facial foramina by the dorsal part of the postorbital arcade.

The postorbital process also separates the trigeminal and facial foramina from the orbit in Tamiobatis vetustus and "Tamiobatis sp. In "Tamiobatis sp." (AMNH 2140), the facial, trigeminal, and anterodorsal lateral line nerves all left the braincase behind the postorbital process (Schaeffer, 1981: fig. 23C, E, $\mathrm{F})$, and the anterodorsal lateral line nerve may have accompanied the trigeminal as postulated in Cladodoides. Accordingly, the supposed trigemino-facial recess in "Tamiobatis sp." probably did not house these nerves any more than in Cladodoides, although it probably housed the external rectus muscle. It is therefore concluded that these Paleozoic sharks lacked a trigemino-pituitary recess like that described by Allis (1914, 1923a) in neoselachians, or even a trigeminofacial recess of the kind envisaged by Schaeffer (1981)

In fact, Orthacanthus is the only extinct shark investigated by Schaeffer (1981) which may have a neoselachian-like trigemino-pituitary fossa (fig. 20B). It definitely possessed a deep recess within the orbital end of the jugular canal, just below a large opening for the combined trigeminal and facial nerves 

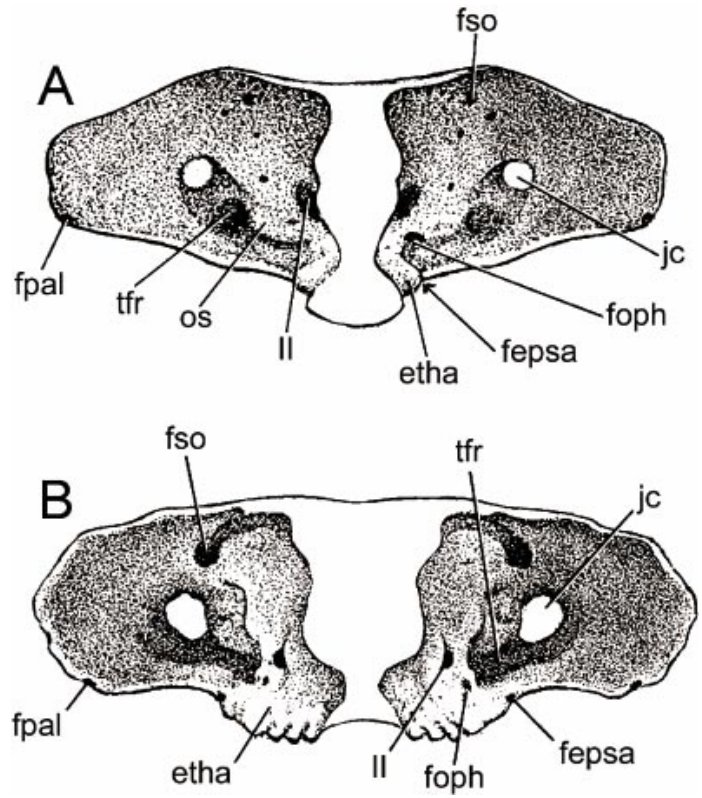

Fig. 20. Diagrammatic views of the postorbital process looking posteriorly, in Cladodoides (A) and Orthacanthus (B). Both panels are from Schaeffer (1981), whose original abbreviations are as follows; etha, ethmoid articulation (= orbital articulation in this work); fepsa, foramen for efferent pseudobranchial artery; foph, foramen for ophthalmic artery; fpal, foramen for branch of palatine nerve (= trigeminal mandibular ramus in this work); fso, foramen for superficial ophthalmic nerve; jc, jugular canal; tfr, trigemino-facialis recess (probably a recess in Orthacanthus, but not in Cladodoides; see text for details); II, optic foramen.

(Maisey, 1983: fig. 14B). Although this recess is positioned farther laterally than the trigemino-pituitary fossa in neoselachians, its proximity to the oculomotor and abducent foramina suggests that it could have housed the external rectus musculature.

The ventral part of the orbit is not preserved in the material referred to Cladodus elegans and nothing is known of its facial and trigeminal arrangement or whether a fossa was present. Coates and Sequeira (1998) identified an opening in the anterior face of the postorbital process in Akmonistion as the trigemino-facial recess and jugular canal. However, they were unable to ascertain the positions of the trigeminal and facial nerves or the pituitary vein and they were uncertain even if it was continuous with the presumed attachment of the optic pedicel. While a jugular canal is almost certainly present in $A k$ monistion, from the above remarks it cannot be assumed that a neoselachian-like trigemino-facial recess was also present, and it is possible that the main facial foramen lay behind the postorbital process as in Cladodoides and "Tamiobatis sp.".

Postorbital Process: There is a massive postorbital process in Cladodoides, with an articular surface for the palatoquadrate posteriorly. The general morphology of the process as described by Gross (1937) and Schaeffer (1981) is well known, but many new features have been revealed by the CT scan. Its main features can be seen in the earlier views (figs. 1-6, 8). In addition, oblique views of the process are shown in figure 17 and a series of clipping planes through the process is shown in figure 19.

A postorbital process similar to that in Cladodoides is present in many Paleozoic sharks (e.g., Orthacanthus, Tamiobatis vetustus, "Tamiobatis sp.", Akmonistion). In one of the fragments referred to Cladodus elegans there is a small area of cartilage apparently forming the floor of the jugular canal (presumably representing cartilage within the embryonic lateral commissure), suggesting that the postorbital process was as well developed here as in Cladodoides and Tamiobatis vetustus. Very little is known about the postorbital process in "Cladodus" hiassicus, although Stensiö (1937) depicted a curious forked distal extremity which suggests the presence of a canal passing through the process. A postorbital process is present in early chondrichthyans such as Pucapampella and Doliodius, but its morphology is quite variable. In Pucapampella, the postorbital arcade is thin and delicate (Maisey, 2001b), but in Doliodus it is more robust and much deeper anteroposteriorly (Miller et al., 2003). Coates and Sequeira (1998) drew attention to the apparently greater length of the postorbital process in Akmonistion and Cladoselache than in many other fossil sharks. They suggested that this may be correlated with presence of a wide orbital roof. However, if the supraorbital cartilage is omitted from consideration, the relative width of the otic region is also a potentially important factor since it is 
approximately twice the width of the interorbital part of the braincase in Cladodoides, Orthacanthus, Tamiobatis vetustus, Hybod$u s$, and many neoselachians, but it is only slightly wider than the interorbital region in Akmonistion. A comparatively narrow otic region can emphasize the lateral extent of the postorbital process, while a wider otic region behind the process would have the opposite effect. Nevertheless, postorbital process length is not consistently related to the width of the otic region, and considerable variations in its length and proportions are found in living and fossil elasmobranchs.

Schaeffer (1981: 48) characterized the postorbital process in Paleozoic sharks as "bounded anteriorly by the trigeminal foramen and posteriorly by the foramen for the hyomandibular nerve". However, that view is no longer tenable since the trigeminal foramen in Cladodoides as well as in Tamiobatis vetustus and "Tamiobatis sp." is clearly located behind the process and does not lie in the orbit (see above).

The postorbital articulation for the palatoquadrate in Cladodoides is directed posteriorly, with a distinct groove flanked laterally by a flange projecting from the posterior rim of the postorbital process as in Orthacanthus, Tamiobatis vetustus, "Tamiobatis sp.", and Akmonistion (Hotton, 1952; Schaeffer, 1981; Coates and Sequeira, 1998). No articular surface is preserved in any of the braincase fragments referred to Cladodus elegans, but there is a postorbital articulation on the palatoquadrate. A postorbital articulation is present in Pucapampella, oriented almost vertically on the thin lateral wall of the postorbital arcade (Maisey, 2001b). The palatoquadrate is still articulated to the postorbital process in one of the Doliodus specimens described by Miller et al. (2003). Although no details of the postorbital articulation are known, the overall arrangement seems similar to that in Cladodoides and many other Paleozoic sharks.

It is now widely accepted that the main anteroposterior passage through the postorbital process in Paleozoic sharks is the jugular canal (see discussion in Schaeffer, 1981). Holmgren (1941) considered that the canal in Cladodoides was homologous to a passage for the otic lateral line nerve in
Chlamydoselachus and Heptranchias, but that view is not widely accepted. Holmgren $(1940,1941)$ nevertheless pointed out that the neoselachian jugular canal only contains the lateral head vein and the main (hyomandibular) trunk of the facial nerve (although the extent to which the latter is "contained" by the canal depends on the extent of the lateral and prefacial commissures as discussed below). Thus, any interpretation involving additional nerves and/or vessels within this canal in extinct elasmobranchs would represent a difference from modern forms.

In Cladodoides, the jugular canal does not pass straight through the postorbital process. Instead, its orbital opening is situated in the ventral part of the orbit, behind the pituitary vein foramen (fig. 19F). The canal then turns posterodorsally and opens posteriorly adjacent to the trigeminal foramen. The posterior end of the jugular canal is therefore positioned farther dorsally than the anterior end. Stensiö (1937: fig. 7) showed that the medial wall of the jugular canal follows a similar course in the Humboldt Museum braincase, although its dorsal part is not preserved. In Tamiobatis vetustus, "Tamiobatis sp.", and Cladodus elegans, the jugular canal is also curved upward posteriorly. A jugular canal is also present in Pucapampella (Maisey, 2001b), but is unknown in Gladbachus and Doliodus.

In addition to the jugular canal, the postorbital process in Cladodoides also contains a transversely oriented canal. This opens proximally into the jugular canal and extends laterally through cartilage derived from the embryonic lateral commissure. The canal opens within the orbital surface of the postorbital process farther distally. Gross (1937) identified the proximal opening of this canal in the wall of the jugular canal ("R.b.") and also identified smaller foramina in the posterior wall of the postorbital process ("r.b.") as well as a large opening in the tip of the process ("Öffnung am distalende des Processus postorbitalis"). He did not recognize the full extent of the large passage through the process, but nevertheless suggested that the buccal ramus of the anterodorsal lateral line nerve passed through the postorbital process and gave rise to small ramules, which 
surfaced along a transverse groove on the posterior surface of the postorbital process. The buccal nerve then supposedly exited the process via the distal opening in the process (probably corresponding to the notch in the end of the process in the Humboldt University specimen illustrated by Stensiö, 1937: fig. 1).

The strongest evidence presented by Gross (1937) that the buccal ramus entered the postorbital process is the presence of small openings on its posterior surface, said to have contained buccal ramules. Unfortunate$1 y$, only one of these openings is even faintly suggested in the CT scan and it could not be clearly traced to the main canal within the process. Furthermore, the openings identified by Gross (1937) are located within the main articular surface for the palatoquadrate (as described by Gross, 1938), which is an implausible location for buccal ramules. It therefore seems more likely that any canals emerging here were for small blood vessels associated with the postorbital articulation. This does not preclude the buccal ramus from passing through the postorbital process, but the evidence for buccal ramules is not compelling.

If the buccal ramus of the anterodorsal lateral line nerve entered the postorbital process in Cladodoides as Gross (1937) proposed, it may have been accompanied by the trigeminal maxillary ramus (forming a buccal-maxillary complex, as in many neoselachians). This is suggested by the position of the inner opening of the canal, which is aligned with the exit of the trigeminal/anterodorsal lateral line nerve (fig. 19D-F). The buccal-maxillary complex does not pass through the postorbital process in any modern elasmobranch although some ramules from the buccal ramus may enter the cartilage to supply part of the infraorbital sensory canal above the process (e.g., Chlamydoselachus). Instead, the neoselachian buccal-maxillary complex mostly passes anteriorly (and to some extent ventrolaterally) below the rectus musculature rather than laterally. Therefore, even if the buccal-maxillary complex in Cladodoides did pass through the postorbital process, its subsequent course into the ethmoid region would have been extremely circuitous because the canal emerges in the lateral part of the orbit rather than medially (although it would still be more medial than originally interpreted by Gross, 1937).

It is also possible that the postorbital process contained the mandibular ramus of the trigeminal nerve (either alone or accompanied by the buccal-maxillary complex). In many neoselachians, the proximal part of the mandibular ramus is directed laterally just below or in front of the postorbital process (e.g., Squalus; Marinelli and Strenger, 1959) in more or less the same orientation as the passage in Cladodoides. However, the mandibular ramus in Squatina and squaloids is located anterior to the postorbital process (which includes the lateral commissure) and does not pass through it. In "long-jawed" neoselachians such as Chlamydoselachus, the mandibular ramus passes close beneath the process posterolaterally before continuing posteriorly toward the mandibular joint and across the lateral surface of the palatoquadrate adductor muscle (Luther, 1909; Allis, 1923a). In hexanchiforms (where the palatoquadrate articulates with the postorbital process posteriorly), the mandibular ramus is directed laterally as it passes below and in front of the postorbital process. The ramus then turns posterolaterally below the postorbital articulation (Luther, 1909). Such a course would also be feasible in Cladodoides and would place the mandibular ramus below and in front of the postorbital articulation. Since the trigeminal foramen lies behind the postorbital process, the only alternative would be for the mandibular ramus to pass above and behind the postorbital articulation, which is implausible anatomically even though the postorbital articulation in hexanchiforms is positioned farther dorsally and medially on the process than in Cladodoides.

Alternatively, the buccal ramus (or buccalmaxillary complex) may have entered the orbit directly via the jugular canal. In neoselachians, this canal contains only the lateral head vein and the hyomandibular trunk, but in "Tamiobatis sp." (AMNH 2140) it also contained the dorsal (superficial ophthalmic) component of the anterodorsal lateral line nerve. Conceivably, the buccal ramus may also have passed through the canal farther ventrally.

To summarize, the lateral part of the post- 
orbital process in Cladodoides, Tamiobatis vetustus, and many other Paleozoic sharks probably contained the trigeminal mandibular ramus, perhaps accompanied by the buccal ramus or buccal-maxillary complex, although the latter may have entered the orbit via the jugular canal. The palatine nerve pierced the floor of the portorbital process medial and ventral to the jugular canal. Other interpretations of Paleozoic sharks, showing the facial mandibular ramus or palatine ramus passing through the lateral part of the postorbital process (e.g., Romer, 1964; Schaeffer, 1981; Coates and Sequeira, 1998), are rejected on morphological grounds (see discussion).

In Cladodoides, there is a small foramen in the floor of the postorbital process near its orbital margin fig. 17B). This may have contained a mandibular vein connected to the lateral head vein as Dick (1978) suggested in Tristychius. Although such a vessel is absent in modern sharks, one is present in rays such as Urolophus. Even if it were present in early sharks such as Cladodoides, the lateral extent of the suborbital shelf would have prevented it from passing beneath the postorbital process.

Dorsal AORTAE: The basic arrangement of major cranial arteries in Cladodoides outlined by Gross (1937) and Schaeffer (1981) is confirmed from CT scanning, since those parts of the basicranial circuit which were enclosed by cartilage can be traced through successive sections and regions that lay in well-defined grooves can also be reconstructed with a high degree of confidence. Intervening regions are more problematic, although some can be inferred from the orientation and direction of adjacent canals and grooves. A new reconstruction of the main basicranial circuit in Cladodoides is presented here, mapped onto a ventral view of the braincase (fig. 21).

Below the occipital cotylus, the paired lateral dorsal aortae lay in grooves and were presumably united behind the occiput. The aortae became completely enclosed by cartilage only anterior to the cotylus (between the level of the third and fourth spino-occipital foramina). The aortic canals continue anteriorly within the parachordal cartilage, resurfacing approximately midway between

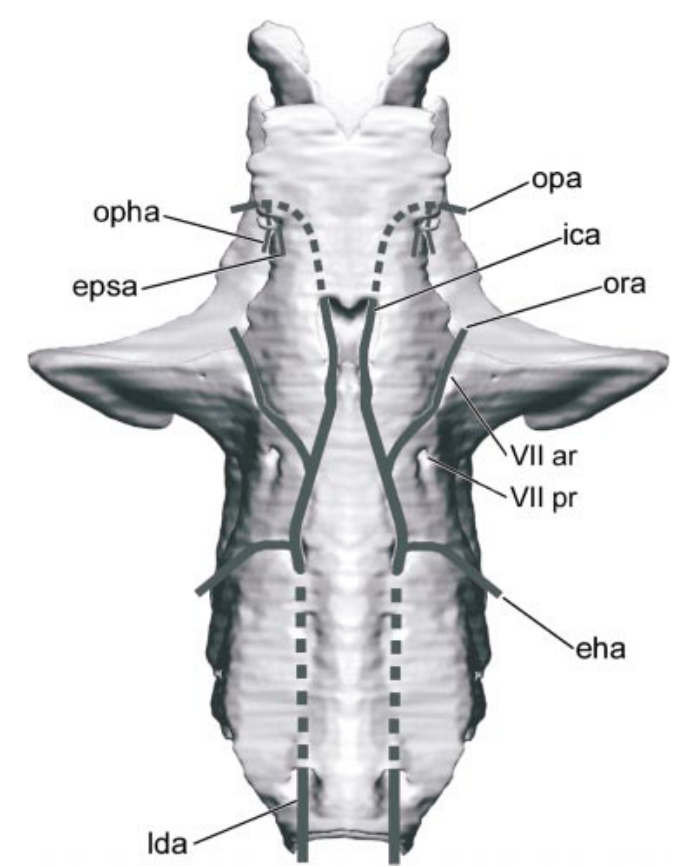

Fig. 21. Ventral view of the braincase in Cladodoides (surface rendering, mirror-imaged), with a reconstruction of the basicranial arterial circuit. No scale.

the cotylus and the bucco-hypophyseal fenestra. There is no evidence of efferent branchial or hyoidean arteries branching from the aortae within the parachordal cartilage. The efferent hyoidean artery probably branched farther anteriorly (see below), while the efferent branchial arteries probably arose farther posteriorly behind the occipital region as in Chlamydoselachus (Allis, 1923a).

The internal carotid and orbital arteries separated only after the aortae left the braincase anteriorly, and their positions are marked by shallow grooves on the basicranium. The internal carotid artery passed into the braincase via the bucco-hypophyseal fenestra, and the orbital artery entered the orbit via a notch or short canal in the suborbital shelf, although the artery was evidently exposed beneath the basicranium and did not pass through cartilage (cf. Schaeffer, 1981: fig. 12C).

Small canals arise from each main aortic canal dorsolaterally and open just below the spino-occipital foramina in the lateral wall of the occipital cotylus (fig. 6). These canals are 
provisionally identified as passages for intervertebral arteries, although it is possible that the anteriormost one contained a vertebral artery. According to Allis (1923a), in Chlamydoselachus the dorsal aorta receives three intervertebral arteries on each side before dividing into paired lateral dorsal aortae; each paired aorta then receives a single intervertebral artery before receiving the anteriormost vertebral artery. Although the aortae and most of the intervertebral arteries in Chlamydoselachus are not contained by cartilage, Allis (1923a: figs. 11, 52) described its anteriormost vertebral artery as traversing the ventrolateral margin of the braincase and he also showed canals for both the first vertebral and intervertebral arteries passing through cartilage on each side of the occiput.

Stensiö's (1937: fig. 6) reconstruction of the basicranial circuit in "Cladodus" hassiacus differs only slightly from the scheme presented here. Its orbital arteries are more completely enclosed by canals than in Cladodoides, and its internal carotids are also enclosed anteriorly. These differences are not necessarily of taxonomic importance, and the Humboldt University specimen may simply be a more mature individual with more extensive calcification. Stensiö (1937) depicted the internal carotids as joining at the midline in his specimen, but this cannot be observed (although scanning might reveal whether these vessels remained separate as in the Senckenberg braincase). The two braincases agree in having: (1) enclosed canals for paired lateral dorsal aortae; (2) the regions where the internal carotid, orbital, and efferent hyoidean arteries divide are exposed ventrally; and (3) the efferent pseudobranchial and ophthalmic arteries join the internal carotids dorsal to the trabeculae.

A single dorsal aorta enters the basicranium in both Falcatus and Akmonistion (Lund, 1985; Coates and Sequeira, 1998), and in a three-dimensional Pennsylvanian braincase referred to "Cobelodus" (Maisey, 2004a and in prep.). Interpretathions of the basicranial circuit in Cobelodus aculeatus by Zangerl and Case (1976) and Schaeffer (1981) are probably erroneous in depicting the aorta as paired and external to the basicranium. While Coates and Sequeira (1998) may be correct that a median groove or canal for the dorsal aorta is primitive for gnathostomes, paired aortic canals are present in Pucapampella and many other Paleozoic chondrichthyans (Maisey, 2001c). In fact, the only chondrichthyans in which a median aortic canal is known are symmoriids and stethacanthids (although the paired lateral aortae are combined within a common sheath in some modern hexanchiforms; Jarvik, 1980). While the character is homoplaseous, within chondrichthyans a single aortic canal may represent a synapomorphy of symmoriid and stethacanthid sharks.

Orbital Artery AND Palatine Nerve: Scanning reveals that what Schaeffer (1981) took to be the posterior opening for the orbital artery in Cladodoides is actually connected internally to the facial (hyomandibular) foramen by a slotlike space and does not reach the expected position of the artery (see above). Gross (1937) suggested that this foramen contained the posterior palatine ramus, which is supported by present observations from the CT scan. It is very unlikely that the orbital artery occupied any part of this canal. Instead, the artery probably ran entirely beneath the braincase before it entered a partially roofed groove (which meets the passage for the anterior ramule of the palatine ramus, immediately below the postorbital process). Furthermore, the CT scan also confirms that the foramen for the orbital artery lies between the two palatine foramina and refutes Schaeffer's (1981) interpretation that it contained the palatine nerve; this passage simply does not connect to other parts of the facial trunk. The orbital and internal carotid arteries in Cladodoides probably did not diverge immediately in front of the anterior opening of the lateral dorsal aorta as suggested by Schaeffer (1981: fig. 12). Instead, the orbital artery arose farther anteriorly and passed anterolaterally across the basicranium to enter the orbit via a short groove beneath the postorbital process (fig. 21). In Cladodoides, the efferent hyoidean artery probably branched from the lateral dorsal aorta somewhere between its emergence from the basicranium and the divergence of the internal carotid and orbital arteries.

As mentioned above, the orbital arteries in the Humboldt University braincase are more 
completely enclosed in canals than in the Senckenberg specimen. The anterior palatine nerve ramule therefore probably entered the orbit within this canal, accompanied by the orbital artery as suggested by Stensiö (1937).

RoOF OF ORBITOTEMPORAL REgION: Gross (1937) noted several small areas where the roof of the braincase is supposedly damaged in the Senckenberg braincase (fig. 2: "Ver"). Scanning reveals that cartilage at the dorsal midline is considerably thinner than elsewhere and is even open locally (fig. 4), leaving a median suture that is especially noticeable in scan sections 85-107 (fig. 12). In neoselachians, the midregion of the braincase roof is one of the last to chondrify (De Beer, 1931), and it may even remain partly open (as an anterior fontanelle) in batoids (Holmgren, 1940). The Cladodoides braincase could represent a juvenile individual in which the roof was only just approaching completion. There is also a distinct opening approximately midway between the parietal and precerebral fossae, resembling a pineal foramen, although it overlies the mesencephalic region of the endocast and therefore seems too far posterior (in neoselachians, the epiphysis usually arises above the optic nerve and reaches the braincase roof above the telencephalon). A similar opening has been described in the roof of the braincase in Acanthodes (Watson, 1937; Miles, 1973; Jarvik, 1977, 1980). This may be related to the pineal organ and is also one of the last regions to ossify (Miles, 1973: 77).

In Cladodoides, the supraorbital shelf is well developed. Paired rows of foramina for ramules of the superficial ophthalmic ramus (supplying the supraorbital sensory canal above the braincase) pierce the cartilage above the orbit. Anteriorly, a notch (still largely obscured by matrix) marks the position where this nerve passed through the supraorbital cartilage and emerged onto the dorsal surface above the olfactory region as in neoselachians (figs. 3, 4). In Tamiobatis vetustus (NMNH 1717), "Tamiobatis sp." (AMNH 2140), and Cladodus elegans, the roof of the orbit is also pierced by numerous foramina for superficial ophthalmic ramules.

In neoselachians, the profundal nerve emerges onto the dorsal surface of the brain- case after leaving the orbit, but this region is not preserved in Cladodoides.

Posterior TeCtUM AND DORSAL FonTANELLE: As in many other fossil sharks, Cladodoides has a large, anteroposteriorly elongated fontanelle in the roof of the braincase (fig. 4). It lies between the otic capsules and is bordered posterolaterally by short, paired dorsal otic ridges (see below). The fontanelle almost reaches the otico-occipital fissure but is separated from it by a weak posterior tectum located below the level of the dorsal otic ridge. The dorsal opening in Cladodoides and primitive chondrichthyans such as $\mathrm{Pu}$ capampella is perhaps best envisaged as a persistent posterior dorsal fontanelle, homologous to that of osteichthyans (see discussion).

A posterior tectum borders the fontanelle posteriorly in Tamiobatis vetustus (NMNH 1717), “Tamiobatis sp." (AMNH 2140), Akmonistion (Coates and Sequeira, 1998), and "Cobelodus" (in prep.). However, in Orthacanthus and Pucapampella, a tectum is absent and the fontanelle is confluent with the otico-occipital fissure posteriorly (Schaeffer, 1981; Maisey, 2001c; Maisey and Anderson, 2001). In adult neoselachians and hybodonts, the otico-occipital fissure is closed, but in many neoselachian embryos a posterior tectum separates the parietal fossa from the embryonic otico-occipital fissure.

Perilymphatic fenestrae have not been identified in Cladodoides. In neoselachians, these fenestrae are located at the upper apex of the posterior semicircular canal. In Cladodoides, this point is located just behind the crus commune, but there is no evidence of an opening here. In Orthacanthus, the chondrified lateral walls of the posterior dorsal fontanelle extend a considerable distance below the surface. Schaeffer (1981: 22) assumed that its perilymphatic openings "must have opened into the bottom of the endolymphatic fossa in close proximity to the posterior semicircular canal." However, as in Cladodoides, there is no evidence that Orthacanthus possessed perilymphatic fenestrae. Moreover, fenestrae in such a deep position could not have had a tympanic function as in neoselachians. If perilymphatic fenestrae were present in Tristychius, as Dick (1978) has suggested, that taxon would be unique 
since its posterior semicircular canal is not separated from the anterior one and a sinus superior is still present.

Endolymphatic foramina have not been identified in Cladodoides although endolymphatic ducts were probably present and presumably passed from the posterior dorsal fontanelle into the unchondrified medial surface of the otic capsule, as hypothesized in some fossil osteichthyans (e.g., Moythomasia; Gardiner, 1984a).

Dorsal OTIC Ridges: Paired dorsal otic ridges are developed on each side of the posterior dorsal fontanelle in several extinct sharks (Schaeffer, 1981). Those of Cladodoides are small and weak, present only adjacent to the fontanelle, and are not directed laterally (figs. 4, 6, 8, 22). The dorsal otic ridges in Akmonistion are also weakly developed and are restricted in their anteroposterior extent (Coates and Sequeira, 1998). They are far more extensive in Orthacanthus, Tamiobatis vetustus, and "Tamiobatis sp.", where they extend from the postorbital process to the posterior end of the fontanelle and their dorsal surface is directed laterally. Dorsal otic ridges are absent in Hybodus and neoselachians. In "Cobelodus", the cartilage surrounding the posterior dorsal fontanelle is raised above the general surface of the braincase, but paired ridges are absent (in prep.).

Lateral Otic Process AND HyomandibULAR FOSSA: Most of the cartilage surrounding the posterior semicircular canals in the Cladodoides braincase is missing, apart from a small fragment illustrated by Gross (1937: fig. 3, "Rest der fehlenden hinteren oticalwand"). A lateral otic process (sensu Schaeffer, 1981) was probably present in Cladodoides, but its original extent is unknown and the large process reconstructed by Schaeffer (1981: fig. 25) is entirely speculative. Note that the lateral otic process is only one of several different processes that may be developed on the lateral wall of the otic capsule (see discussion).

According to Gross (1937), the lateral wall of the otic capsule in Cladodoides includes an articular fossa for the hyomandibula although its extent was not clearly established. Schaeffer (1981) showed that the hyomandibular fossa in Orthacanthus is confined to a small area on the posterolateral wall of the otic capsule and involves only the lateral otic process and lateral otic ridge. This is confirmed in articulated Orthacanthus specimens from Germany (Heidtke, 1998, 1999). The hyomandibular fossa in the Senckenberg braincase was probably restricted to the same region, but the specimen is damaged on both sides in the expected position of the fossa, exposing the posterior semicircular canals.

General Features of Skeletal LabyRINTH: The skeletal labyrinth in Cladodoides is seen in views of the endocast (figs. 2326). The medial capsular wall is unchondrified and the vestibular chamber is therefore confluent with the cranial cavity. The horizontal semicircular canal lies just below the lateral otic ridge, and the anterior and posterior semicircular canals meet dorsally at a crus commune, below which is a short sinus superior. The sinus merges proximally with the cranial cavity and vestibular chamber, and features deeper within the vestibular region cannot be discerned. There is a prominent utricular recess (confluent with the saccular chamber medially) to which the anterior and external ampullae are connected. The posterior ampulla is confluent with the saccular chamber and there is no evidence of a preampullary canal (sensu Maisey, 2001b). The main vestibular chamber is weakly divided into dorsal (utricular) and ventral (saccular) regions and there is little evidence of a lagenar chamber. It is unknown whether the crista neglecta was enlarged as in neoselachians, although this seems unlikely. The medial (nonampullary) part of the external semicircular canal turns inside the curve of the posterior canal before passing deeper into the labyrinth cavity. Its subsequent course cannot be determined but presumably it entered the sacculus just below the crus commune, as in chimaeroids and osteichthyans. The anterior and external ampullae in Cladodoides are clearly separated and probably had separate openings into the utricular recess. A short passage for the anterior ramus of the octaval nerve passes through cartilage forming the anteroventral part of the labyrinth wall before entering the proximal end of the utricular recess. Presumably, the anterior and posterior rami were already separated and the posterior ramus passed through an unchondrified part of the capsular wall. 
The only other fossil shark in which the position of the octaval nerve has been described is Cobelodus aculeatus (Zangerl and Case, 1976: figs. 8, 9), where it was apparently accompanied by the facial and trigeminal nerves within a recess that may be homologous to the acustico-trigemino-facial recess in neoselachians (see discussion).

The semicircular canals of the Senckenberg braincase are arranged in a profoundly different way from the neoselachian-like pattern described by Stensiö (1937) in the Humboldt University specimen. Crucially, Stensiö (1937) argued for an almost completely separate posterior semicircular canal in the latter, forming a ring that communicates only with the vestibular region. If that is true, the Humboldt University braincase represents the oldest known shark with such an arrangement (a neoselachian-like labyrinth morphology is also present in Mesozoic hybodonts; Maisey, 2004a and in prep.).

The semicircular canals in Cladodoides are comparatively narrow, as in Squalus. By contrast, the canals in Notorynchus are much wider (fig. 27B, C), while those of Orthacanthus seem to fall somewhere in between (Schaeffer, 1981: fig. 14). Canal diameter may be correlated generally to behavior, since fishes with low turning speeds tend to have larger semicircular canals than highly maneuverable fishes (Young, 1981). This suggests that Cladodoides was perhaps as agile as Squalus in swimming maneuvers.

The anterior and posterior ampullae in Cladodoides lie at approximately the same distance from the midline. However, in Squalus and Notorynchus, the posterior ampulla is closest to the midline (Maisey, 2004b: fig. 11E). The functional significance of this difference is unknown but may be related to decoupling of the posterior canal from the rest of the labyrinth.

OCCIPITAL ARCH: Scanning reveals that the occipital region in the Cladodoides braincase is far more complete than was previously supposed (Maisey, 2001c). In dorsal view, the occipital arch intrudes only slightly between the otic capsules (although it also extends behind them) and there is a persistent otico-occipital fissure similar to that described in Orthacanthus, Tamiobatis vetustus, "Tamiobatis sp.", and Akmonistion
(Schaeffer, 1981; Coates and Sequeira, 1998). The occipital arch contains the foramen magnum and also forms a continuous roof to the dorsal nerve cord. By contrast, in Tamiobatis vetustus and "Tamiobatis sp.", the dorsal part of the occipital arch anterior to the foramen magnum is uncalcified (Schaeffer, 1981). However, the margins of the calcified region are unfinished, suggesting that a complete membranous occipital arch was present even though its dorsal part was unmineralized. Schaeffer (1981) argued that the glossopharyngeal nerve of Orthacanthus and Tamiobatis vetustus passed through a slitlike opening in the ventrolateral surface of the otic region (the ventral otic notch, corresponding to the metotic fissure in embryonic neoselachians). In Orthacanthus, the ventral otic notch extends anteriorly medial to the posterior semicircular canal. It then passes completely beneath the hyomandibular fossa and terminates more or less level with the middle of the posterior dorsal fontanelle. In the original Tamiobatis vetustus braincase (NMNH 1717), the ventral otic notch also extends below the hyomandibular fossa, but it terminates somewhat farther posteriorly than in Orthacanthus (Schaeffer, 1981: fig. 19). The occipital region is not preserved in the Humboldt University braincase. Among the material referred to Cladodus elegans is a complete occipital segment with a persistent otico-occipital fissure and no signs of an uncalcified dorsal area.

Although the fissure is persistent in Cladodoides, its metotic part is distinctly widened to form a glossopharyngeal canal, floored by a continuous shelf (presumably derived from the embryonic hypotic lamina) below the otic capsule. The arrangement resembles that of neoselachians, except that in Cladodoides the canal is still connected to the otico-occipital fissure dorsally, instead of being completely isolated. Laterally, the occipital arch is connected to the parachordal region by the posterior basicapsular commissure and is not fused to the posterior wall of the otic capsule. The dorsal part of the oticooccipital fissure is narrow, but it widens just medial to the posterior ampulla in the expected position of the vagal nerve (fig. 28A, C). Thus, in Cladodoides the positions of the glossopharyngeal and vagal nerves are both 


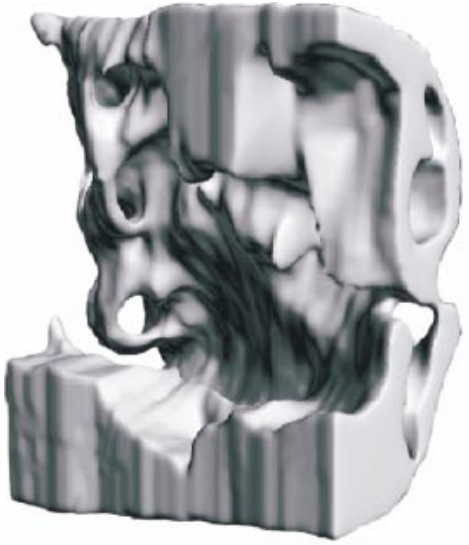

A
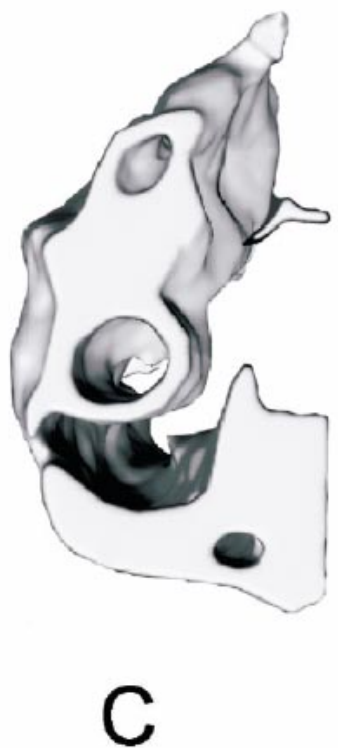

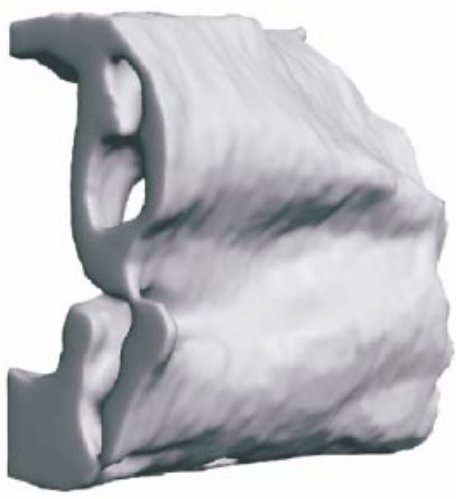

B

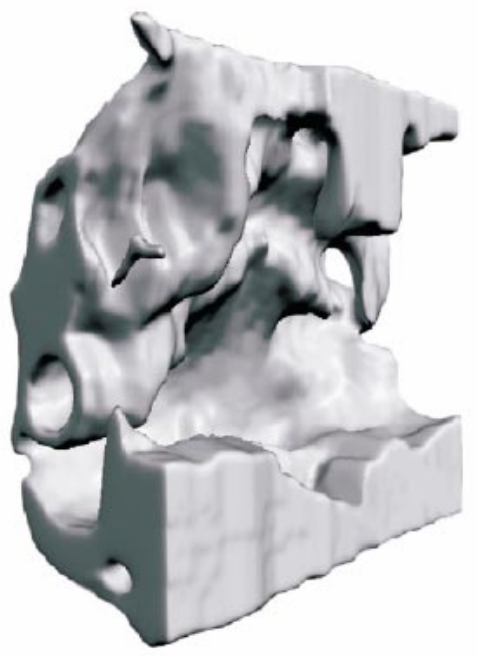

Fig. 22. Otic region of the Cladodoides braincase (surface rendering), oblique perspective detail views. (A) Anteromedial view; (B) anterolateral view; (C) posterior view; (D) posteromedial view. This block overlaps slightly with that depicted in figure 17, repeating the exit of the hyomandibular trunk. Note the absence of a medial wall to the saccular chamber, utricular recess, and crus commune, although the semicircular canals are almost completely enclosed by cartilage. The position of the octaval (acusticovestibular) nerve is indicated, but is more clearly seen in sections (see fig. 18E). The hypotic lamina extends beneath the otic capsule, forming a wide glossopharyngeal canal and a short metotic fissure. The lateral dorsal aorta is enclosed by cartilage posteriorly, then emerges ventrally. The uneven internal floor and thickened cranial roof in front of the posterior dorsal fontanelle are artifacts of digital interpretation, as the braincase wall is poorly calcified in these areas; it is unlikely that the synotic tectum was originally as thick as shown here. 

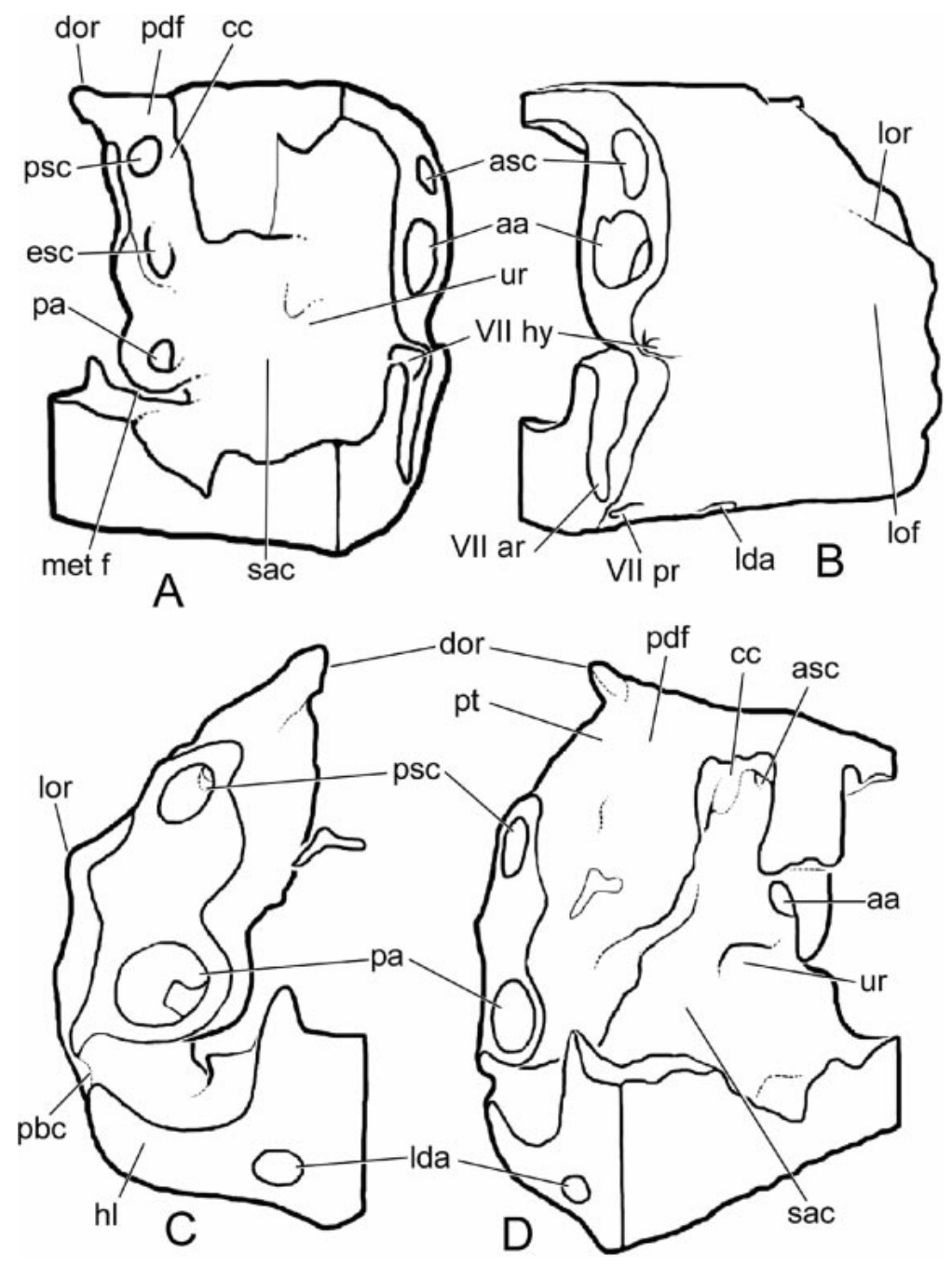

Fig. 22. Continued.

much better defined within the otico-occipital fissure than in Orthacanthus. In Cladodoides, the ventral otic notch is either absent or extremely short, unlike in Orthacanthus where there is an extensive notch (Schaeffer, 1981: fig. 6). The lateral margin of the hypotic lamina in Cladodoides therefore meets the otic capsule posteriorly as in neoselachians and hybodonts.

In Cladodoides, the glossopharyngeal canal merges with the lumen of the saccular chamber, but there is no evidence that the nerve actually entered it; instead, they were probably separated by a membranous capsular floor as in Scyliorhinus (De Beer,
1931). In Orthacanthus, most of the hypotic lamina is separated from the capsule by a wide ventral otic notch. However, in "Tamiobatis sp." (AMNH 2140), there is only a short ventral otic notch which does not extend anteriorly beyond the level of the hyomandibular fossa. The arrangement in "Tamiobatis sp." is therefore more like Cladodoides than Orthacanthus. It is unknown whether a metotic fissure was present in the Humboldt University braincase.

Cladodoides has a circular occipital cotylus which includes a deep conical central cavity tapering into a short notochordal canal. The canal extends only a short distance 


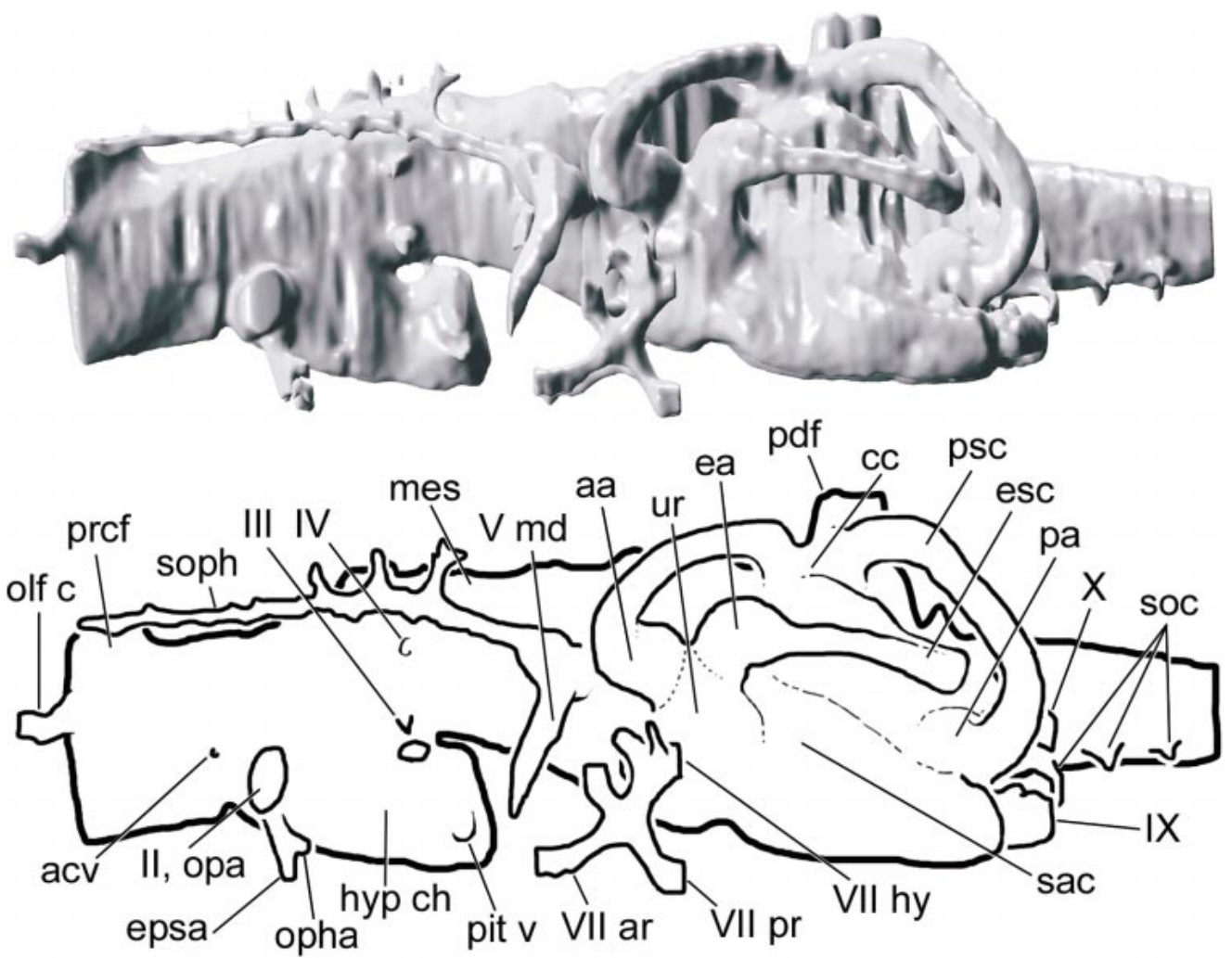

Fig. 23. Lateral view (left side) of the Cladodoides endocast (surface rendering).

into the parachordal region and does not reach the dorsum sellae (figs. 7, 28B, C). The inner surface of the cotylus appears smooth in the reconstruction (fig. 8), although this may be an artifact generated by the imaging software. In Hybodus, this surface is formed in fibrocartilage, with a rough spongy appearance (Maisey, 1987). The cotylar surface also has a spongy appearance in other Paleozoic sharks. In modern sharks, the cotylus includes an occipital "hemicentrum" or "half centrum" (Maisey, 1984a, 1984b), with a smooth inner surface like those of the amphicoelous chordal centra forming the vertebral column. This smooth surface characterizes those neoselachians in which (1) the fibrous notochordal sheath is invaded by mesenchymatous cells to form chordal centra and (2) the notochord extends into the basicranium. Both these conditions are met in modern sharks and presumably were also realized in extinct forms where a hemicentrum is present (e.g., Palaeospinax, Synechodus;
Maisey, 1984b, 1985; Maisey et al., 2004). In batoids, chordal centra are typically present but a hemicentrum is absent and the notochord terminates within the synarcual cartilage some distance behind the occipital arch. Thus, the presence of an occipital hemicentrum in neoselachians is probably correlated with their tendency to develop chordal centra. Such centra are absent in hybodonts and many Paleozoic sharks, where the hemicentrum is also absent. The occipital region in gnathostomes is always perichordal, forming outside the elastica externa (like the paired arcualia of the vertebral column; Goodrich, 1930: 10). The cotylus in extinct sharks such as Cladodoides is therefore almost certainly of perichordal origin and is probably homologous to the perichordal cartilage surrounding the chordal occipital hemicentrum in modern sharks.

In Cladodoides, at least two foramina for intervertebral arteries pass through the lateral wall of the cotylus, suggesting that the co- 


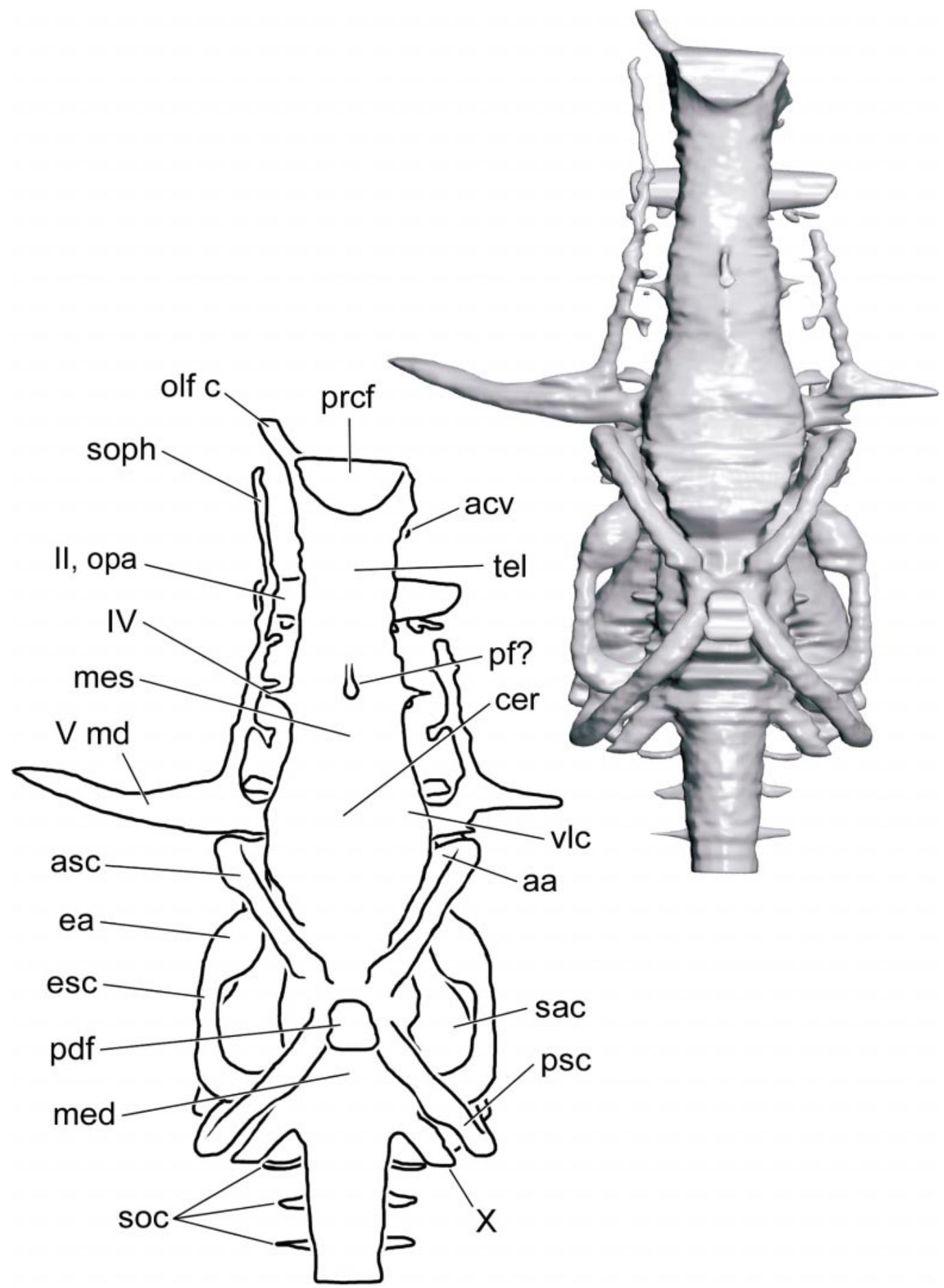

Fig. 24. Dorsal view of the Cladodoides endocast. Anterior to top. 


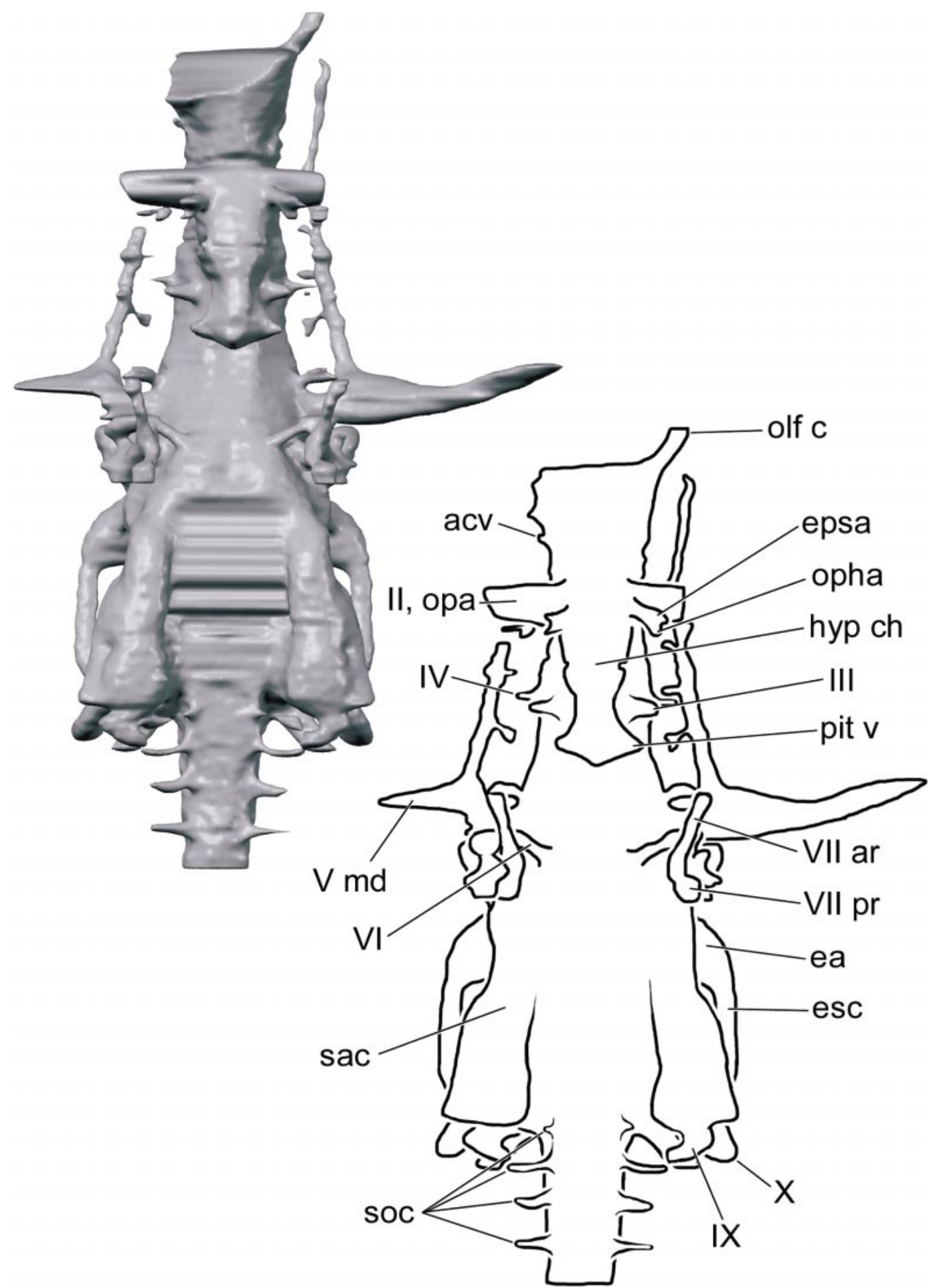

Fig. 25. Ventral view of the Cladodoides endocast. Anterior to top. 


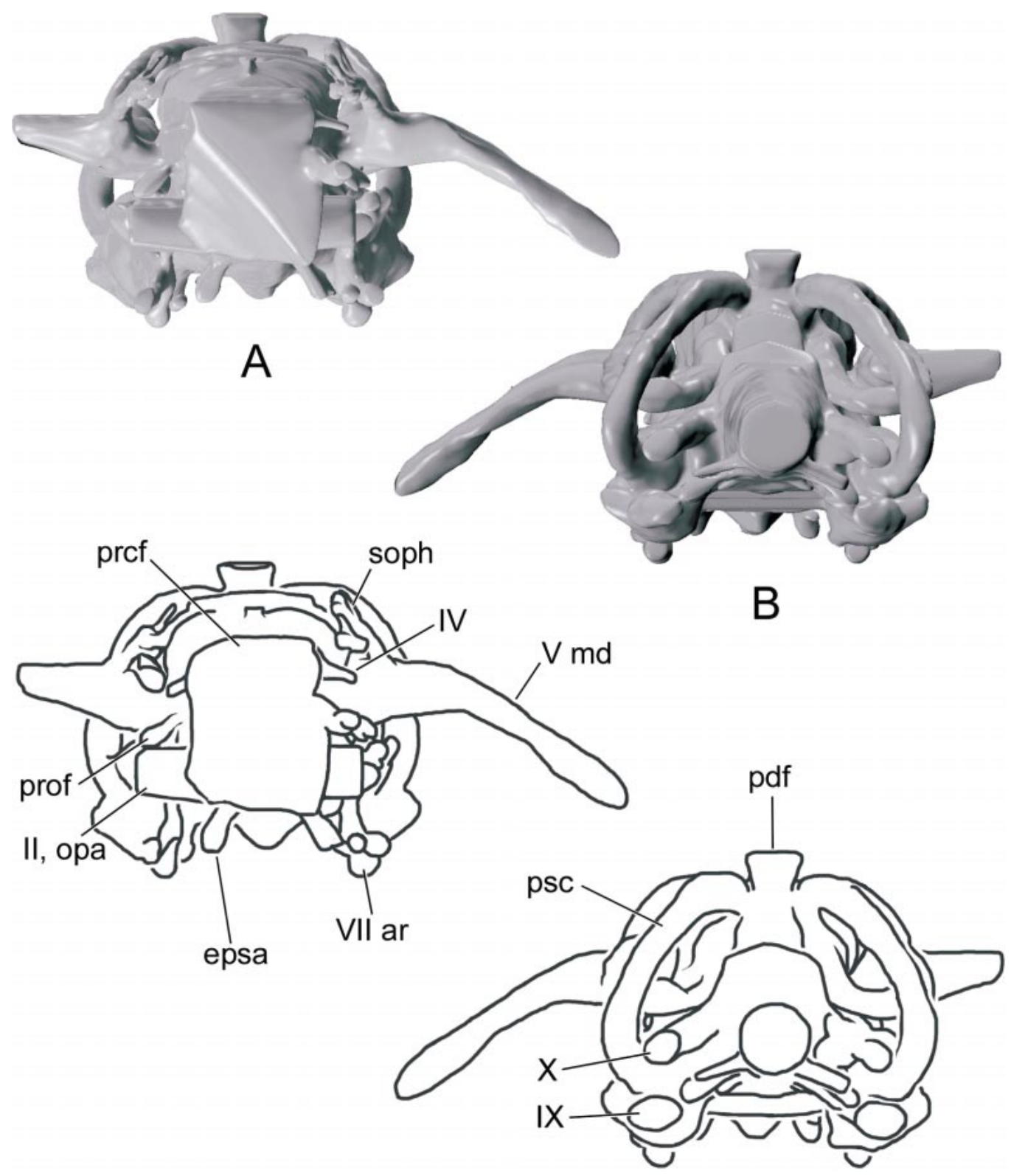

Fig. 26. Anterior (A) and posterior (B) views of the Cladodoides endocast.

tylar region spans more than one vertebral segment. The presence of four or five spinooccipital foramina farther dorsally suggests that the occipital arch included several vertebral segments.

The occipital cotylus and notochordal canal in Cladodoides lie within the thickness of the basicranial cartilage at the presumed line of fusion between the left and right parachordals (fig. 7). The parachordal cartilage therefore completely enclosed the notochord, as in Scyliorhinus and Squalus (De Beer, 1931; El-Toubi, 1949; see fig. 29 here). Cartilage surrounding the notochord in Cladodoides is calcified (presumably perichordally), although surrounding areas of the basal 


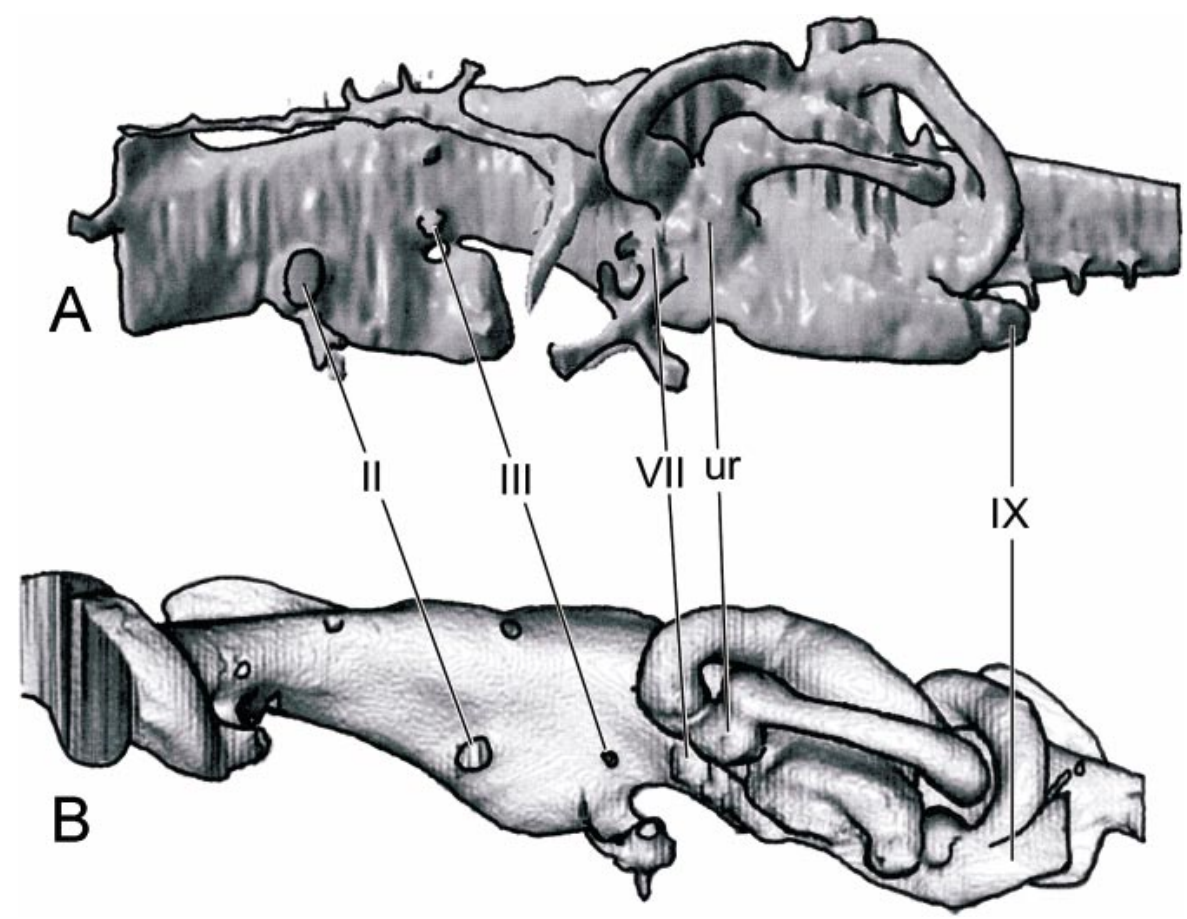

Fig. 27. Comparison of the cranial endocast in Cladodoides (A, D) and Notorynchus (B, C). The endocasts are positioned with their anterior ampullae aligned. Note the greater length of the mesencephalic chamber in Cladodoides, resulting in a more anterior location of the hypophyseal chamber and foramina for the oculomotor and optic nerves and efferent pseudobranchial artery. Despite these differences, the position of the trochlear foramen is similar, suggesting that the proportions of the mesencephalic chamber primarily involve its ventral part and may be related to differential development of the polar cartilage. Not to scale.

plate are unmineralized. Posteriorly, the occipital cotylus completely separates the parachordals, and the notochordal canal at its center is located a short distance above the floor of the braincase. As the cotylus tapers anteriorly, the calcified sheath of the notochordal canal remains elevated but is still connected to the floor of the basal plate by a vertical septum. This represents a region of deep but localized endochondral calcification beneath the notochord and presumably lies along the original line of fusion between the paired parachordals (fig. 30A, B). On each side of the septum is a small uncalcified "chamber" (probably cartilage-filled in life). Farther anteriorly, the median septum breaks down and these chambers are interconnected, separating the calcified notochordal canal from the floor of the basal plate (although the canal probably remained within the thickness of the parachordal cartilage; fig. 30C).
A similar arrangement is seen in a fragmentary occipital region of a large Tamiobatis- or Ctenacanthus-like braincase (USNM 299647; fig. 31), but here the notochordal canal is considerably elevated above the floor of the parachordal plate by a median septum and there are massive paired subnotochordal chambers. In the holotype of Tamiobatis vetustus (NMNH 1717), a subnotochordal septum and paired chambers are revealed where the basicranial cartilage has been stripped away by erosion (see Romer, 1964: pl. 1). The posterior part of a short median notochordal septum is also visible in the slabbed "Tamiobatis sp." braincase, immediately anterior to the occipital cotylus (AMNH 2140; Schaeffer, 1981: fig. 24H). Externally, the position of the subnotochordal chambers is marked by paired convex areas lying between the median groove and the lateral aortic grooves. These features conceivably be- 


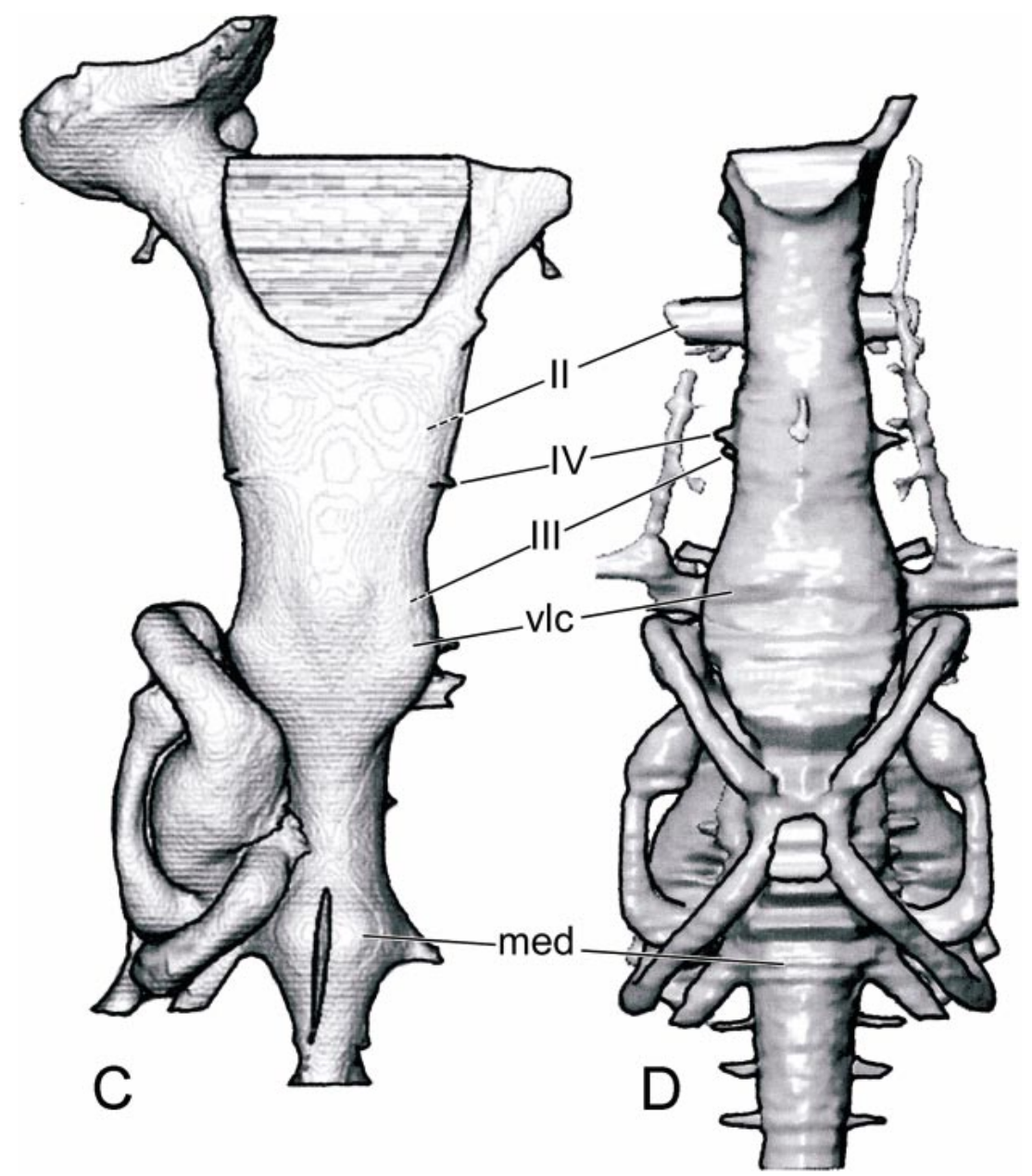

Fig. 27. Continued.

came enhanced during growth and may be better developed in larger individuals. According to Schaeffer (1981: fig. 9), in Orthacanthus the notochord is contained entirely within the thickness of the mineralized basal plate and no subnotochordal septum or chambers are present (as in neoselachians). Thus, a subnotochordal sheath and chambers may not characterize all early sharks with an elongate otico-occipital region.

In Cladodoides, at least four and perhaps five spino-occipital foramina are present along each side of the occipital arch (figs. 27, 28). At least four spino-occipital nerves are also present in the occipital block of "Tamiobatis sp." (Schaeffer, 1981: fig. 24F) and there may even be an additional pair farther anteriorly, passing through the parachordal cartilage between the floor of the cranial cavity and the metotic fissure. Three or four spino-occipital foramina are present along each lateral surface in "Cobelodus" (in prep.) and in an undescribed isolated occipital region referred tentatively to Cladodus elegans. Paired foramina are also present in the parachordal region of another fragment referred to $C$. elegans, corresponding to the more anterior ones in "Tamiobatis sp." In these specimens, the cartilage containing the anteriormost foramina is probably homologous to the dorsal process of the parachordal plate in neoselachians, which forms the basicapsular commissure connecting the hypotic lamina to the floor of the otic capsule (e.g., 


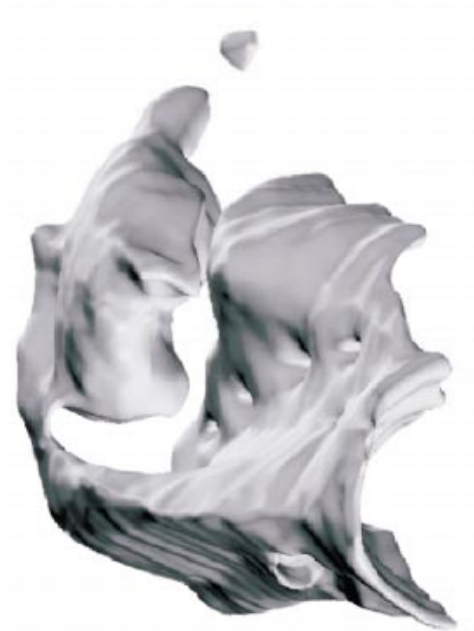

A

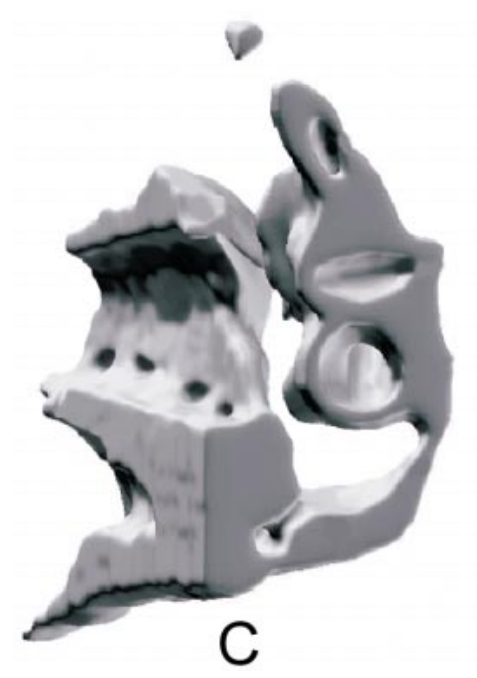

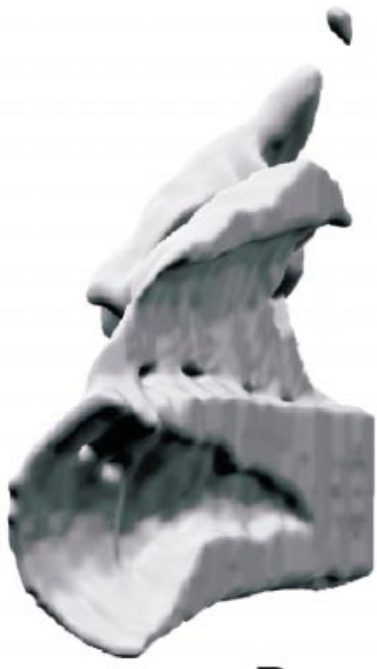

B

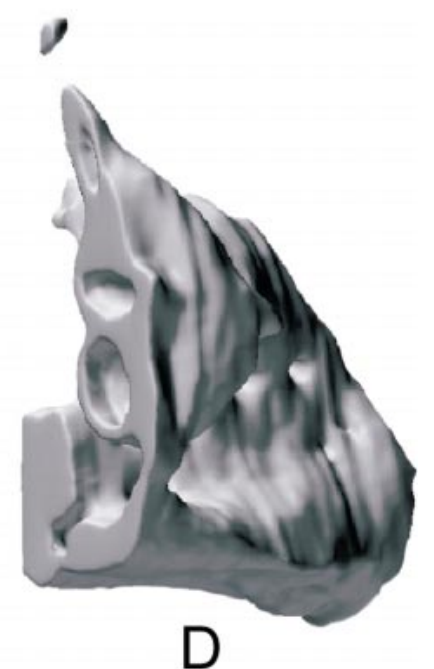

Fig. 28. Occipital region of the Cladodoides braincase (surface rendering), oblique perspective detail views: (A) posterolateral view; (B) posteromedial view; (C) anteromedial view; (D) anterolateral view. This block overlaps slightly with that shown in figure 22, repeating the tip of the dorsal otic ridge and posterior semicircular canal. The hypotic lamina is fused to the capsular wall laterally, forming a glossopharyngeal canal. The position of the vagal nerve is indicated by an expansion in the otico-occipital fissure above the glossopharyngeal canal.

Scyliorhinus; De Beer, 1931). The glossopharyngeal trunk passes through the dorsal process in neoselachians, but in "Tamiobatis sp." and Cladodus elegans it presumably entered the metotic fissure farther anteriorly.

It is concluded that three to five spino-occipital nerves were commonly present in $\mathrm{Pa}-$ leozoic sharks, and this may represent the primitive number for elasmobranchs.

CRAnial Endocast: Gross (1937) was able to observe very little of the cranial cavity or skeletal labyrinth in Cladodoides, although he was able to determine the positions of the posterior semicircular canals 

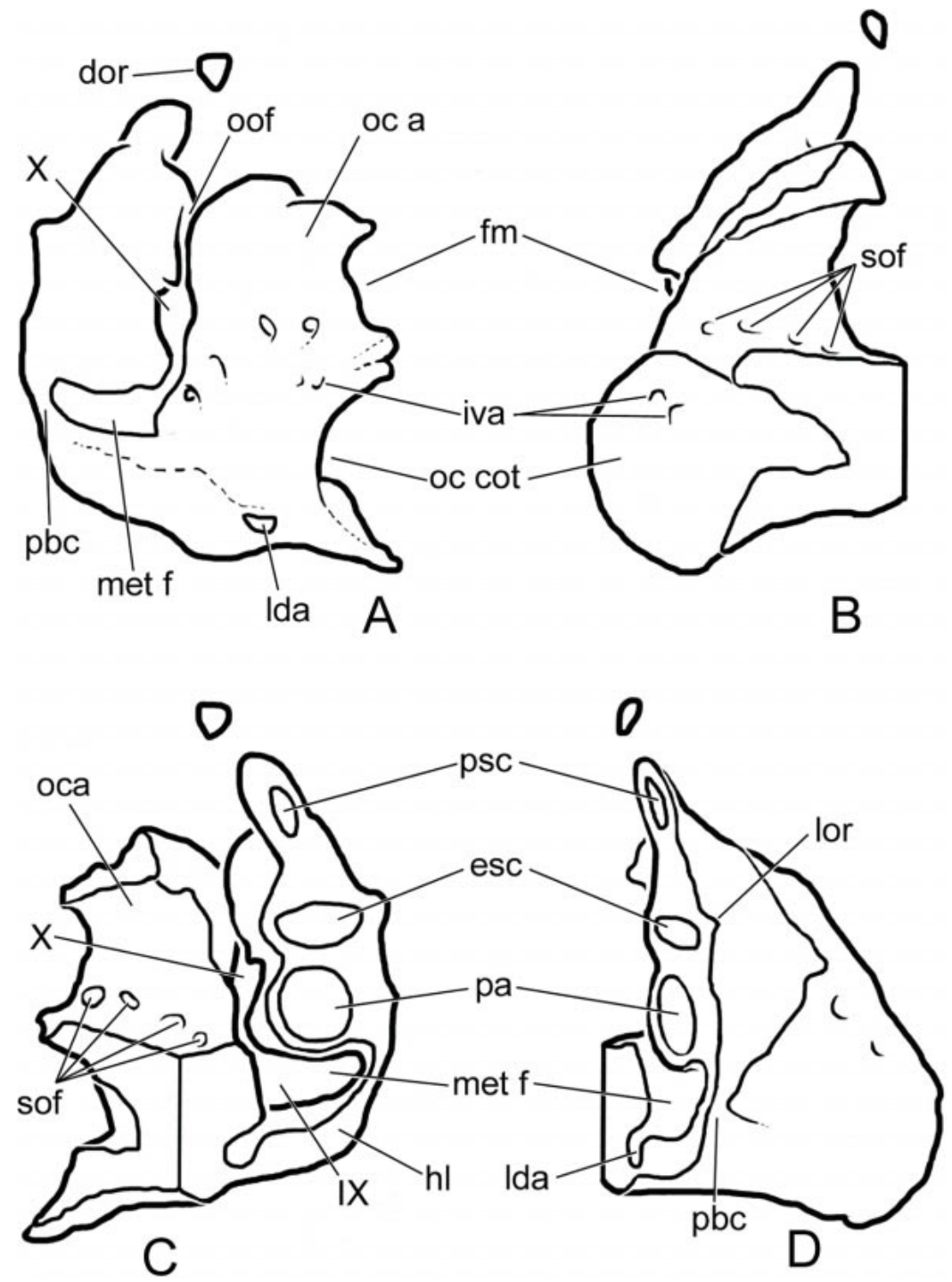

Fig. 28. Continued.

(which are partially exposed) and the horizontal semicircular canals beneath a horizontal crista on the lateral capsular wall. The cranial and labyrinth spaces have been reconstructed digitally from surface renderings, as outlined at the beginning of this paper (figs. 23-26). Lateral and dorsal views of the endocast in Cladodoides and modern Notorynchus are shown in figure 27.

The telencephalic region is narrow from side to side, but is quite deep and merges imperceptibly with the diencephalic and mesencephalic regions, as in neoselachians (figs. $23,24)$. A small protuberance on the dorsal surface marks the position of a small opening in the roof of the braincase. It is tempting to regard this as a pineal organ, but its posterior location suggests that it merely represents an unchondrified area of the cranial roof. The combined exit for the optic nerve and artery is located anterodorsal to the conjoined efferent pseudobranchial and ophthalmic ca- 


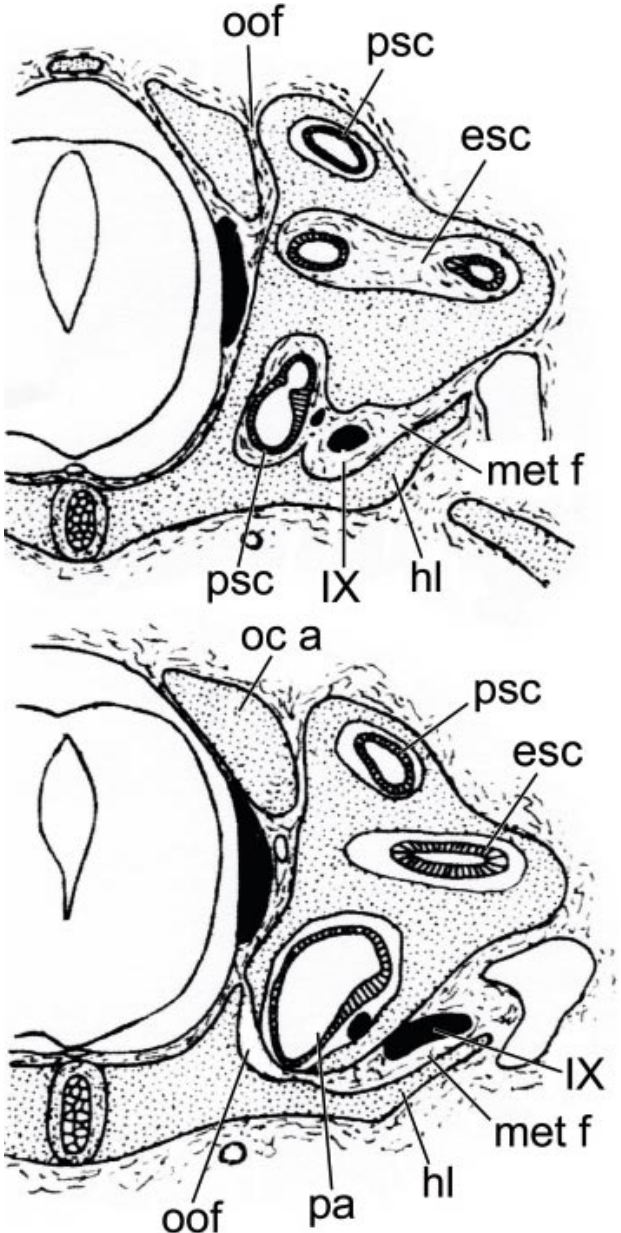

Fig. 29. Transverse sections of a Scyliorhinus embryo (after De Beer, 1931; no scale indicated). Note the continuity of the metotic and otico-occipital fissures beneath the otic capsule, and the position of the glossopharyngeal nerve between the capsule and hypotic lamina.

nals and lies immediately in front of a small transverse constriction marking the position of the presphenoid ridge.

The mesencephalic region widens posteriorly and the cerebellar chamber is well defined in lateral and dorsal views (figs. 23, 24). In Cladodoides, "Tamiobatis sp.", and Orthacanthus, the mesencephalic chamber is comparatively longer than in Squalus or Notorynchus. In Cladodoides, the cerebellar chamber is broadly expanded, forming paired vestibulolateral (auricular) chambers. As in Squalus and Notorynchus, these chambers

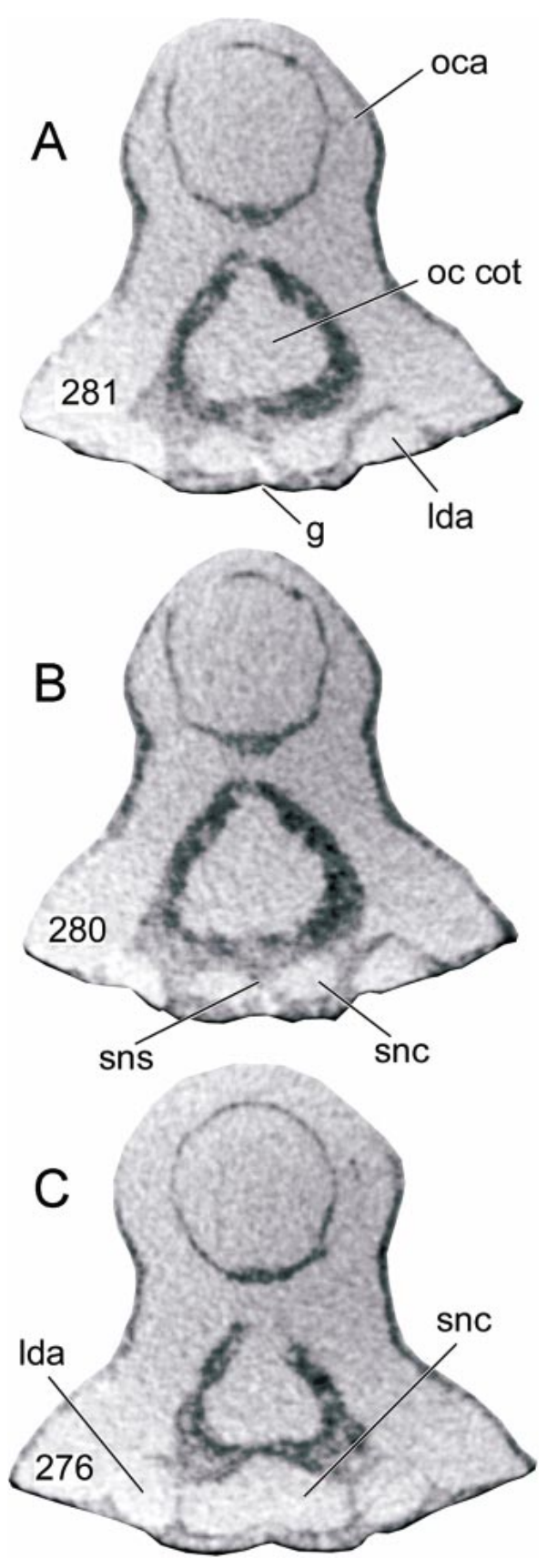

Fig. 30. Transverse sections through the occipital region of the Cladodoides braincase showing the subnotochordal septum and "chambers" (presumably cartilage-filled in life). (A) Slice 281; (B) slice 280; (C) slice 276. All of these features extend over fewer than 10 slices (approx. $3 \mathrm{~mm}$ ) in this braincase, in contrast with the occipital region shown in figure 31 . 

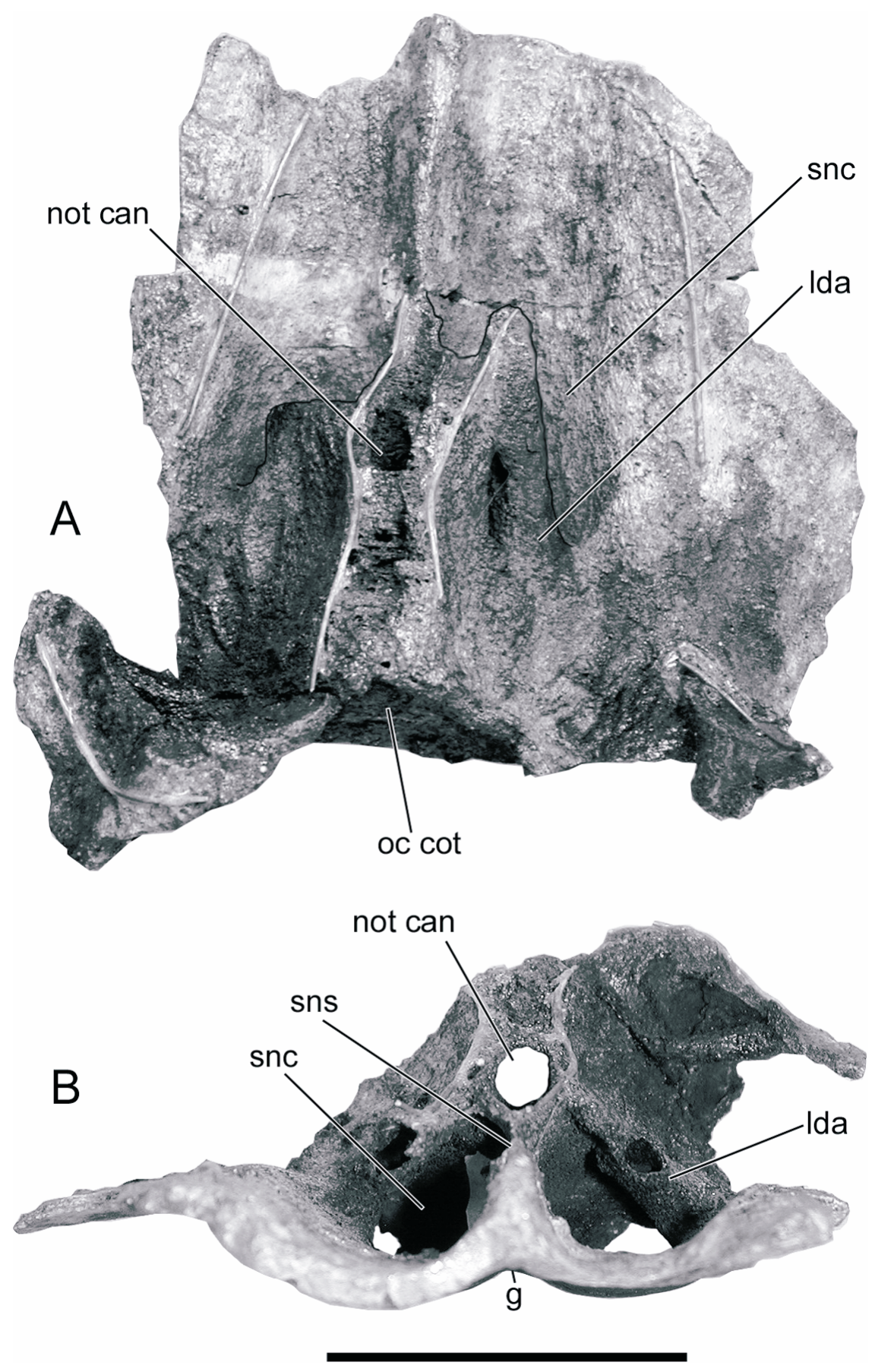

Fig. 31. Fragmentary acid-prepared occipital region of an undetermined (ctenacanth?) shark braincase, USNM 299647, St. Louis Limestone, Alton, Illinois. (A) Dorsal view, anterior to top; (B) anterior view. Note the extensive notochordal sheath supported by a median subnotochordal septum and large paired "chambers" forming prominent convexities ventrally between the median groove and paired aortic canals. Scale bar $=5 \mathrm{~cm}$. 
are seen best in dorsal view (fig. 24). By contrast, the roof of the cerebellar chamber in Chlamydoselachus slopes more abruptly above the acustico-trigemino-facial recess, and its vestibulolateral chambers are more evident in lateral aspect (Allis, 1923a: fig. 12). In Hydrolagus, modern osteichthyans (e.g., Protopterus, Salmo), and lampreys, the cerebellum and fourth ventricle are commonly situated behind the level of the pituitary organ (Jollie, 1962). The adenohypophysis and oculomotor nerve were apparently located some distance anterior to the otic capsule in early chondrichthyans (e.g., Pucapampella; Maisey, 2001b) and in some placoderms (e.g., Tapinosteus; Stensiö, 1963a). Collectively, these observations suggest that the morphology of the cerebellum in Cladodoides and Orthacanthus was generalized, and that the comparatively "short" mesencephalic region in hybodonts and neoselachians is an apomorphic feature.

In neoselachians, the trochlear canal arises from the optic lobe (which may be well developed, as in Squalus, or weak, as in $\mathrm{No}$ torynchus; Maisey, 2004b). The trochlear canal presumably marks the position of the optic lobe in Cladodoides although the cranial cavity around it is not expanded and optic lobes are not evident.

The scan does not show the full extent of the endocast between the otic capsules. The medullary region consequently cannot be described in detail, although it was evidently constricted between the capsules as in neoselachians. The posterior dorsal fontanelle becomes indistinct as it merges laterally with the crus commune below the anterior and posterior semicircular canals. The vagal nerve probably occupied the widest part of the otico-occipital fissure (figs. 25, 26B). Farther ventrally, this fissure is connected with the glossopharyngeal canal as discussed earlier. The transverse passage for the trigeminal mandibular ramus inside the postorbital process projects laterally from the endocast; its proximal region has been reconstructed as traversing the jugular canal and arising at the trigeminal foramen. The superficial ophthalmic complex passes through cartilage forming the roof of the jugular canal. Presumably, this cartilage formed either in the lateral commissure or between it and the primary postorbital process arising from the supraorbital cartilage. The trunk of the superficial ophthalmic complex then must have connected with that of the mandibular complex just above the posterior end of the jugular canal. Four or five paired spino-occipital nerves are present, although the anterior ones are obscured by the vagal nerve except in ventral view (fig. 25). The spino-occipital nerves are paired, as in gnathostomes generally and unlike in cyclostomes and many extinct agnathans where an alternating pattern is observed.

The hypophyseal (pituitary) chamber can be seen in lateral and ventral views of the endocast (figs. 23, 25). The chamber is defined posteriorly and dorsally by the very extensive dorsum sellae. Its lateral wall contains the pituitary foramen, as in gnathostomes generally (fig. 7). The chamber is much larger than in modern sharks, where the dorsum sellae projects only a short distance above the floor of the cranial cavity. As in modern sharks, however, the roof of the hypophyseal chamber curves above the pituitary foramen and meets the medial surface of the sidewall of the cranium just below the oculomotor foramen, confining the dorsum sellae entirely behind the efferent pseudobranchial foramen. It is concluded that the dorsum sellae in Cladodoides probably arose from the embryonic polar cartilage as in modern sharks, and the considerable size of the hypophyseal chamber and dorsum sellae in Cladodoides supports the suggestion that the polar cartilage made an extensive contribution to the basicranium (see above and the following discussion). In modern sharks, the hypophyseal chamber is long and is bounded posteriorly by the dorsum sellae and anteriorly by the preclinoid wall. In batoids, there is no dorsum sellae, preclinoid wall, or pituitary fossa, although there is a membranous pituitary sac below which there is a subpituitary space containing the internal carotid arteries (Gegenbaur, 1872; Allis, 1928).

In Cladodoides, the anterior margin of the dorsum sellae separates the hypophyseal and mesencephalic chambers almost directly below the oculomotor foramen as in neoselachians, but its oculomotor passage is located much farther anteriorly relative to the trigeminal nerve and utricular recess (this can be 
seen by comparing the endocasts of Cladodoides and Notorynchus; fig. 27). The size of the hypophyseal and mesencephalic chambers in Cladodoides may therefore reflect the greater extent of the polar cartilage-derived region, since the oculomotor foramen is located farther anteriorly not only to structures behind the level of the hypophysis (e.g., the trigeminal foramen) but also to features farther dorsally (e.g., the trochlear foramen). In Hybodus and Tribodus, the mesencephalic and hypophyseal chambers are proportioned as in modern sharks (Maisey, 2004a: fig. 5). The mesencephalic chamber in Orthacanthus is quite long although the polar cartilage apparently made a smaller contribution to its floor than in Cladodoides (Schaeffer, 1981: fig. 14).

A weak lateral hypophyseal ridge extends from the dorsum sellae as far as the optic foramen along the inner wall of the cranial cavity in Cladodoides. This ridge separates the lateral wall of the cranial cavity from the upper margin of the hypophyseal chamber, as in some neoselachians (fig. 7; also see Maisey, 2004b). The anterior margin of the hypophyseal chamber is weakly defined by a low transverse presphenoid ridge $(=$ praesphenoïdvorsprung; Gegenbaur, 1872). A similar ridge is present in some neoselachians (e.g., Heptranchias, Squalus, Dalatias, Deania, Mustelus) but not in others.

The hypophyseal chamber of Orthacanthus is much shallower than in Cladodoides, although its dorsum sellae is almost as prominent (Schaeffer, 1981: fig. 14). The extent of these structures have not been determined in Tamiobatis vetustus, but there is a large hypophyseal chamber in "Tamiobatis sp." The dorsum sellae and hypophyseal chamber are both very deep in "Cobelodus" (Maisey, 2004a: fig. 8), and the dorsum sellae extends much farther dorsally in modern chimaeroids than in neoselachians (e.g., Callorhinchus; De Beer, 1937: pl. 21).

\section{DISCUSSION}

Ontogenetic Aspects of the BRAincase: The Cladodoides braincase probably represents an individual just approaching its full ontogenetic maturity, but which may still have been a small, submature shark and could potentially have grown to a much greater size. Although the braincase is essentially complete, there is some evidence that it was not fully developed. Ontogenetic studies of the neoselachian braincase (e.g., De Beer, 1931; Holmgren, 1940) suggest that the narrow openings along the dorsal midline and the large bucco-hypophyseal fenestra in Cladodoides are juvenile or submature features. Absence of a precarotid commissure behind the bucco-hypophyseal fenestra is another possible juvenile feature. Absence of a chondrified medial capsular wall might be an ontogenetically primitive condition, but the wall is unchondrified even in much larger (presumably adult) Paleozoic sharks (e.g., "Tamiobatis sp.").

The absence of multilayered prismatic calcification may represent a juvenile characteristic, but there is no modern paradigm (multiple-layered calcification is rarely found in neoselachians and typically only in highstress regions of the jaws; Summers, 2000). Nevertheless, multiple-layered calcification occurs throughout the braincases of Orthacanthus and many other Paleozoic sharks. Such calcification may have developed cumulatively with age, and the presence of successive prism layers in such forms suggests that calcification was locally periodic rather than gradual. There is little evidence of subsequent in vivo resorption or disruption of the laminar arrangement once formed, although the layers are often broken up postmortem. Adult Cladodoides may therefore have been more heavily calcified than the small specimen investigated. If multiple layers of prismatic cartilage were developed in Cladodoides, this presumably commenced only after the braincase was fully formed. On the other hand, multiple-layered calcification is also absent or poorly developed in other much larger Paleozoic sharks (e.g., Cladoselache), so it is possible that Cladodoides never possessed more than a single layer of prisms. It is not possible to infer any phylogenetic significance to the absence of multiple-layered calcification in Cladodoides without a greater understanding of its wider phylogenetic distribution and development in elasmobranchs generally.

Comparison with neoselachian embryos nevertheless indicates that in most respects 
the ontogenic development of the Cladodoides braincase is fairly complete. For example, the postorbital process seems to be fully developed and the palatine ramus is enclosed within its floor, so ontogenetic fusion of the lateral commissure to the rest of the braincase was well advanced. In addition, most of the metotic fissure in Cladodoides is closed, apart from a small notch extending from the ventrolateral margin of the glossopharyngeal canal. Consequently, most of the hypotic lamina is fused to the capsular floor as in adult neoselachians, rather than leaving an elongate ventral otic notch as in Orthacanthus. The interorbital septum is essentially complete and the embryonic prootic foramen is absent. Moreover, the embryonic basicranial cartilages (trabeculae, polar cartilages, parachordals) are completely fused together, and an extensive contribution to the basicranium by the polar cartilage is suggested by the position of the orbital articulation and efferent pseudobranchial artery, by the extent of the hypophyseal chamber and dorsum sellae, and by the position of both the prefacial commissure and the facial foramen behind the orbit (discussed further below).

Persistence of an otico-occipital fissure throughout ontogeny may be a conserved feature of crown-group gnathostomes. In Cladodoides, much of the embryonic metotic fissure is presumably closed, and the hypotic lamina/basicapsular commissure is inferred to have enclosed a wide glossopharyngeal canal below the otic capsule. The otico-occipital fissure in Cladodoides is therefore less extensive than in much larger (presumably adult) Orthacanthus braincases in which the embryonic metotic fissure persisted as a ventral otic notch. The metotic fissure in Cladodoides presumably closed early in ontogeny, as in neoselachians, although its embryonic otico-occipital fissure was persistent farther dorsally. From a phylogenetic perspective, therefore, ontogenetic closure of the metotic fissure may have preceded obliteration of the dorsal otico-occipital fissure. Closure of the metotic fissure precedes fusion of the occipital arch to the otic capsules in some neoselachians (e.g., 62-mm Heterodontus; Holmgren, 1940). However, no consistent pattern is discernible and in 64-mm Squalus the lateral rudiments of the arch (the occipital pi- lae) are already fused to the capsules before the occipital arch is complete dorsally (ElToubi, 1949), implying some heterochrony in development of the occipital arch and closure of the otico-occipital fissure in neoselachians. The occipital arch is complete in Cladodoides (and in 62-mm Heterodontus), suggesting that the dorsal part of the otico-occipital fissure persisted later in ontogeny than in Squalus.

PRECEREbral Fontanelle: The presence of a precerebral fontanelle has been regarded as an elasmobranch synapomorphy (Maisey, 1984a; Gaudin, 1991), although some (e.g., Holmgren, 1942; Schaeffer, 1981) have suggested that the fontanelle is homologous to the ethmoid canal in holocephalans. However, the ethmoid canal is an enclosed tube containing the anterior portion of the profundal nerve dorsal to the olfactory capsules and is apparently a secondarily roofed-over extracranial space (De Beer and Moy-Thomas, 1935; Didier, 1995), not a remnant of the cranial cavity as proposed by Holmgren (1942). It is nevertheless possible that absence of a precerebral fontanelle in chimaeroids is secondary, and that its presence is a chondrichthyan synapomorphy (Coates and Sequeira, 2001a). A precerebral fontanelle is present in Gladbachus and Doliodus (Heidtke and Krätschmer, 2001; Miller et al., 2003) but has not been observed in Pucapampella (although its ethmoid region has not yet been described; Maisey, 2001b; Maisey and Anderson, 2001).

EFFERENT PSEUdobranchial ARTERY: In neoselachians, the efferent pseudobranchial and internal carotid arteries meet inside the cranial cavity, dorsal to the trabecular-polar plate (De Beer, 1924). Usually, the ophthalmic and efferent pseudobranchial arteries branch within the orbit, and there is only a single exit for the combined vessel in the orbital wall (e.g., Chlamydoselachus; Allis, 1923a: fig. 52). In chimaeroids, the efferent "pseudobranchial" artery is specialized to provide the main vascular supply to the brain (e.g., Callorhinchus; De Beer and MoyThomas, 1935). The efferent vessel is uninterrupted by a pseudobranch, and the spiracle closes early in ontogeny, while internal carotid and ophthalmic arteries are absent in the adult. In the primitive Devonian chon- 
drichthyan Pucapampella, the efferent pseudobranchial artery apparently passed through the basicranium via a canal which opens near the posterior margin of the optic foramen, but there is no evidence of a separate ophthalmic foramen, suggesting that it met the efferent pseudobranchial within the orbit (Maisey, 2001b). Nothing is known of their arrangement in Gladbachus or Doliodus.

In modern osteichthyans, the efferent pseudobranchial and internal carotid arteries are conjoined below the trabeculae and enter the basicranium together. The same arrangement has been identified in primitive extinct actinopterygians (e.g., Ligulalepis; Basden and Young, 2001). It is unlikely that such a subcranial commissure existed in Pucapampella and the two vessels probably passed through the trabecular cartilage separately (Maisey and Anderson, 2001). Stensiö (1963a) and Goujet (1984) argued that the efferent pseudobranchial arrangement in placoderms resembled that of elasmobranchs rather than osteichthyans, but Young (1980, 1986) has presented evidence that in placoderms the artery probably passed medially in a basicranial groove and joined the internal carotid before it entered the braincase. Consequently, the most parsimonious scenario is that the efferent pseudobranchial artery primitively passed through the trabeculae in gnathostomes, and this arrangement is conserved by osteichthyans and primitive chondrichthyans such as Pucapampella (Holmgren, 1943; Young, 1986; Maisey and Anderson, 2001). Unification of the internal carotid and efferent pseudobranchial arteries dorsal to the trabeculae is apparently an apomorphic condition shared by neoselachians and many extinct sharks including Cladodoides, Orthacanthus, and Hybodus. Although it has been claimed that the efferent pseudobranchial in modern chimaeroids is located above the trabeculae as in elasmobranchs (Schaeffer, 1981), the condition in Callorhinchus as described by De Beer and Moy-Thomas (1935) suggests that it is located at the lateral margin of the trabeculae, an arrangement which could have been derived from that found in Pucapampella, since the efferent pseudobranchial in Callorhinchus also enters the braincase via a short canal (supposedly at the former boundary of the trabeculae and pal- atoquadrate). The configuration of the efferent pseudobranchial in neoselachians and chimaeroids may therefore represent two distinct apomorphic patterns, neither of which primitively characterizes chondrichthyans. Thus, a connection between the internal carotid and efferent pseudobranchial arteries dorsal to the trabeculae is interpreted here as an apomorphic feature of elasmobranchs but not all chondrichthyans.

Anterior Palatoquadrate ArticulaTION: In modern elasmobranchs, there may be no anterior connection between the palatoquadrate and braincase (e.g., batoids). Where an articulation is present, however, two distinct patterns are recognizable. In one pattern, either an articular surface is present in the ethmoid region (e.g., Heterodontus, orectolobiforms), or the palatoquadrate has a ligamentous attachment to the braincase in the corresponding position (e.g., Scyliorhin$u s$ ). In the other pattern, the dorsal margin of the palatoquadrate bears an orbital process extending a considerable distance into the orbit and usually resting against an articular surface located between the optic and efferent pseudobranchial foramina (e.g., Chlamydoselachus, hexanchiforms, squaloids, squatinoids, and pristiophoroids; Maisey, 1980).

Holmgren (1942: 140) compared the anterior palatoquadrate articulation in Cladodoides and Chlamydoselachus and noted that while both are positioned anteriorly on the palatobasal shelf, their topographic relationship to the optic nerve differs. He concluded that Cladodoides "seems to have behaved as a squaloid shark, but with a shelf (crista subocularis) developed behind the orbital articulation.' Maisey (1980) and Schaeffer (1981) recommended restricting the term orbital articulation to the pattern found in modern orbitostylic sharks. However, while this recommendation certainly emphasizes the derived nature of their anterior palatoquadrate articulation, it does little to resolve its possible homology with "ethmoidal" and "orbital" articulations in other neoselachians or in elasmobranchs generally. Additionally, there has been uncertainty as to whether any of the anterior palatoquadrate articulations in sharks are homologous to those found in other gnathostomes, such as the palatobasal ar- 
ticulation of osteichthyans or the "orbital" and "basal" articulations in placoderms.

Turning first to the question of homology between ethmoidal and orbital articulations in elasmobranchs, De Beer (1931) and Holmgren (1941: char. 47) considered that an orbital process was present in many modern and extinct elasmobranchs, including galeomorphs and all orbitostylic sharks. However, the articulation in Heterodontus and galeomorphs does not correspond topographically with the orbital articulation in orbitostylic sharks. According to De Beer (1931, 1937) and Holmgren (1940), the orbital process in Heterodontus and Scyliorhinus is confined to the anterior part of the trabecular region, and any articular surface is developed far anterior to the polar cartilage and to the efferent pseudobranchial artery (e.g., Heterodontus, orectolobiforms). By contrast, in orbitostylic sharks (e.g., Squalus, Etmopterus), the articular surface is located at the junction of the trabecular and polar cartilages and lies immediately anterior to the efferent pseudobranchial foramen (Maisey, 1980). Holmgren $(1940,1942)$ found evidence for two adjacent embryonic palatoquadrate connections in this part of the orbit in Squalus, one with the posterior end of the trabecular plate (ultimately forming the orbital articulation) and another (which ultimately disappears) with the polar cartilage slightly farther posteriorly. The articulation in Heterodontus and galeomorphs lies on the trabecular margin but has no obvious connection to the polar cartilage and is located much farther anteriorly than the orbital articulation in orbitostylic sharks. Arguably, these articulations are not homologous on both topographic and ontogenetic grounds. Support for that view is found in the different ontogenetic origins of the cartilage connecting the palatoquadrate to the braincase; in Scyliorhinus, a separate chondrification arises from the trabecular cartilage (the "lateral trabecular process" of Holmgren, 1940) and fuses with the palatoquadrate to form the orbital process, but in Squalus the orbital process arises as part of the palatoquadrate (Holmgren, 1940: 252).

Holmgren (1940: 253) argued that the reason the orbital articulation is located farther anteriorly in Scyliorhinus is simply because the trabeculae do not "grow out very much anterior to the process, whereas in the squaloids the growth principally takes place frontally", implying that migration of the articulation has occurred in squaloids. However, the presumptive articulations initially do not form in the same place and their relationships to the polar cartilage are completely different from their earliest appearance. The articulation in Scyliorhinus first appears beneath the anterior part of the orbit (Holmgren, 1940: fig. 103), whereas the orbital articulation in Squalus and Etmopterus arises beneath its posterior part. Importantly, neither of these articulations shows appreciable anteroposterior relocation within the orbit during subsequent ontogeny (Holmgren, 1940: figs. 55, $67,79,81)$. Thus, apart from the fact that both articulations are formed on the lateral margin of the trabeculae, there is little to suggest that they are homologous to each other.

In addition, these articulations differ in their topographic and ontogenetic relationships to the suborbital cartilage. Holmgren (1940: 253) noted that in Scyliorhinus, "the membrane connecting the palatoquadrate with the trabecula medially chondrifies, forming the subocular cartilage or shelf. In Squalus and Etmopterus no such shelf is present, the connecting membrane never chondrifying". Note, however, that the cartilage forming the shelf in Scyliorhinus is posterior to the articulation, whereas the "connecting membrane" in Squalus is anterior to it. Thus, even if the articulation is homologous in these taxa, the "connecting membranes" differ topographically. The anterior articulation in Scyliorhinus resembles that of Cladodoides in lying anterior to the suborbital shelf, and in both cases the orbital artery penetrates the shelf posteriorly. The articular surface in Cladodoides therefore resembles the orbital articulation of orbitostylic sharks in its relationship to landmark arterial vessels and presumably to the polar cartilage, but also resembles the ethmoidal articulation in galeomorphs and Heterodontus in its topographically anterior location within the orbit and the presence of a suborbital shelf (penetrated by the orbital artery) behind the articulation.

In Hybodus basanus, there is extensive lateral contact between the braincase and the anterior part of the palatoquadrate (Maisey, 1983). However, only the anterior part of this 
contact actually forms an articular surface in the ethmoid region, a considerable distance both from the efferent pseudobranchial foramen and from the presumed site of the polar cartilage. In the primitive neoselachian Synechodus, a prominent articular surface for the palatoquadrate occupies the anterior part of the orbit in front of the optic and efferent pseudobranchial foramina, as in Heterodontus and galeomorphs, but in Synechodus a suborbital shelf is absent and there is no evidence of any palatoquadrate articulation in the inferred site of the polar cartilage (Maisey, 1985: figs. 3, 4). The suborbital shelf extends for a considerable distance anterior to the efferent pseudobranchial foramen in $\mathrm{Hy}$ bodus basanus (Maisey, 1983).

Thus, in elasmobranchs there may be (1) an articular surface situated immediately anterior to the efferent pseudobranchial foramen and to the inferred anterior margin of the polar cartilage-derived region (either confined to the orbital floor, as in Cladodoides and Orthacanthus, or extending into the orbit behind the optic foramen as in Chlamydoselachus); (2) an articular surface situated farther posteriorly in the orbit, but still associated with the efferent pseudobranchial artery and located behind the optic foramen (e.g., Squalus, Notorynchus); or (3) an anteriorly situated articular surface that is not associated with either the efferent pseudobranchial foramen or with the polar cartilage (e.g., modern Heterodontus and galeomorphs, and perhaps Synechodus and Hybod$u s)$. A fourth (nonarticular) condition is represented by fully hyostylic elasmobranchs, in which an ethmoid or orbital articulation is absent (e.g., batoids, Tribodus). These observations suggest that the anterior palatoquadrate articulation in Paleozoic sharks such as Cladodoides, Tamiobatis, and Orthacanthus is primitively homologous to the orbital articulation in modern orbitostylic sharks, although the orbitostylic condition is probably derived in extending farther dorsally into the orbit and behind the optic foramen (Maisey, 1980). However, there is little evidence to suggest homology of these articulations to the anterior one in Heterodontus, galeomorphs, Synechodus or Hybodus.

De Beer (1931) and Schaeffer (1981: 56) both claimed that an ethmoid articulation is present in Chlamydoselachus, anterior to the orbital articulation and medial to the olfactory capsule and ectethmoid process. Actually, no real articulation is present here; instead (according to Allis, 1923a: 167), the anterior part of the palatoquadrate in Chlamydoselachus merely "rests, when the mouth is closed, against the lateral edge of the solum nasi". Holmgren (1941) also did not recognize a separate ethmoid articulation in Chlamydoselachus. In fact, the membranous anterior connection between the palatoquadrate and braincase described by De Beer (1931) in Scyliorhinus does not form a cranio-quadrate articulation and probably consists only of connective tissue, as in $\mathrm{No}$ torynchus (Wolfram, 1984; Maisey, 2004b). In Orthacanthus, the anterior part of the palatoquadrate has a shallow depression dorsally (Hotton, 1952). In articulated specimens, this depression is located below the olfactory capsule (Schaeffer, 1981: fig. 1). A similar depression is also found on other Paleozoic shark palatoquadrates (including material referred provisionally to Cladodus elegans). In Hybodus basanus, a short flat area on the corresponding part of the palatoquadrate makes contact with the ventral surface of an ethmopalatine process on the internasal plate (Maisey, 1983). This may correspond to the "rostral articulation" in Orthacanthus (Schaeffer, 1981: 56), but probably neither is homologous to the orbital articulation in neoselachians. At best, the anterior depression may have formed a sliding surface (e.g., Orthacanthus) or a buttress (Hybodus) beneath the olfactory capsule like that described by Allis (1923a) in Chlamydoselachus. It may be homologous to the ethmoidal articulation in galeomorphs and Heterodontus, although in these forms the articulation is with the medial surface of the palatoquadrate rather than with its dorsal surface.

Primitive actinopterygians possess an autopalatine (or "ethmoid"; Janvier, 1996) articulation anteriorly, plus a palatobasal articulation farther posteriorly (e.g., Mimia, Moythomasia; Gardiner, 1984a). The autopalatine articulation in actinopterygians may be homologous to the ethmoidal articulation in chondrichthyans, since it is developed on the lateral margin of the embryonic trabeculae and involves the anteriormost (autopalatine) 
part of the upper jaw. An autopalatine articulation is probably absent in Acanthodes and placoderms (Gardiner, 1984a).

Homology of the elasmobranch orbital articulation to the palatobasal articulation in osteichthyans was first advocated by Huxley (1876) and was supported by De Beer (1931: 628), who argued for a separate ethmoid articulation anterior to the orbital one in some modern sharks (e.g., Chlamydoselachus; but as discussed above, there is actually little evidence of such an ethmoid articulation in Chlamydoselachus, hexanchiforms, or other orbitostylic sharks). De Beer (1931: 419) noted that the elasmobranch orbital (his basal) articulation and the osteichthyan basitrabecular articulation are both located ventral to the lateral head vein and are both dorsal and anterior to the palatine ramus of the facial nerve (the same relationship may also be postulated in Cladodoides; see sections on the jugular canal and palatine ramus later in this work).

Some authors have strongly contested De Beer's proposal (e.g., Holmgren, 1943; Devillers, 1958). Miles (1965, 1973) interpreted Acanthodes as having an osteichthyan-like basipterygoid process and a palatobasal articulation with the palatoquadrate, and he concluded that this was different from the orbital articulation in elasmobranchs. Holmgren's position is somewhat ambiguous, since he has argued that the orbital and palatobasal articulations are not homologous (Holmgren, 1943), but has suggested elsewhere that the autopalatine process in Acanthodes is homologous to the orbital process in squaloids and hexanchiforms (Holmgren, 1942), basing his argument on the supposed presence of an anterior symphysis anterior to the process in Acanthodes (which he regarded as an "almost identical organization of the palatoquadrate" to that of squaloids and hexanchiforms). According to Miles (1965, 1973), however, Acanthodes lacks an anterior palatoquadrate symphysis. Perhaps even more significantly, a new reconstruction of the head in the early chondrichthyan Pucapampella suggests that the palatoquadrates were separated by a wide ethmoid region anteriorly, and that no symphysis was present (Maisey and Janvier, in prep.). If chondrichthyans primitively lacked a palatoquadrate symphysis, similarities noted by Holmgren (1942) in the anterior part of the suspensorium of Acanthodes and sharks are probably convergent.

Gardiner (1984a: 299) drew attention to topographic and ontogenetic similarities between the osteichthyan palatobasal articulation and the neoselachian orbital articulation, noting that "the basipterygoid process is typically developed in osteichthyans, but is also recognizable in many selachians where it forms part of the subocular shelf as in Heptranchias, and in Squalus ... where it arises from the site of the polar cartilages." There are also compelling topographic similarities in the palatobasal articulations of primitive osteichthyans and Pucapampella (Maisey, 2001b; Maisey and Anderson, 2001). In some primitive sarcopterygians, there is a "suborbital ledge" with a palatal articular surface (Yu, 1998). The osteichthyan palatobasal articulation and the elasmobranch orbital articulation are both associated developmentally with the polar cartilage and with the efferent pseudobranchial artery, although in osteichthyans this vessel meets the internal carotids below the basicranium rather than above it as in elasmobranchs (but not necessarily in all chondrichthyans; Maisey and Anderson, 2001: 710). The elasmobranch orbital articulation can therefore be considered homologous to the palatobasal articulation of osteichthyans on the basis of its topographic relationship to the lateral head vein and the palatine ramus of the facial nerve (De Beer, 1931) and to the site of the embryonic polar cartilage (Gardiner, 1984a). It is concluded that (1) a palatobasal articulation is primitively present in Acanthodes, osteichthyans, and chondrichthyans, and therefore probably represents a conserved gnathostome character; (2) it has become highly specialized in modern orbitostylic sharks; and (3) it has been secondarily lost (perhaps independently) in Heterodontus, galeomorphs, and batoids.

It has been claimed that "basal" (i.e., palatobasal) and "orbital" articulations are both present in some placoderms (e.g., Tapinosteus, Kujdanowiaspis; Stensiö, 1963a). Clearly such conjunction would refute homology between them (Miles, 1968). However, Goujet (1984) found no evidence of a 
palatobasal articulation in placoderms and pointed out that the palatoquadrate is confined to an area below the "suborbital" shelf (which includes several structures associated with the orbital cartilage in other gnathostomes; e.g., optic pedicel, eye muscle insertions, and branches of the oculomotor nerve). Furthermore, Stensiö's (1963a: figs. 16, 17, 21, 22) illustrations reveal several features challenging the identity of these articulations. He inferred the anterior limit of the polar cartilage-derived region in Kujdanowiaspis from the supposed position of the efferent pseudobranchial foramen, but this may have been incorrectly identified (see below). In Tapinosteus, the supposed orbital articulation is apparently located even farther anteriorly, on the lateral margin of a region that is presumably derived from the trabecular cartilage (Stensiö, 1963a: fig. 59) and is widely separated from the region supposedly derived from the polar cartilage (like the ethmoidal articulation in galeomorphs and Heterodontus). The supposed basal articulation in placoderms is therefore unlikely to be homologous to the palatobasal articulation in osteichthyans. However, Young (1986: 30) noted that in Buchanosteus there is a close association between one of the cranio-quadrate articulations and the groove for the efferent pseudobranchial artery, which passes between two articular areas (he also recognized a similar arrangement in Romundina). Thus, while there is still considerable disagreement about the articular relations between the palatoquadrate and braincase in placoderms (reviewed by Young, 1986), there may be an articulation associated with the polar cartilage-derived region of the braincase in some taxa.

Coates and Sequeira (1998) suggested that presence of an ethmoidal articulation is an elasmobranch synapomorphy and that the articulation was primitively restricted to the anterior part of the suborbital region (e.g., Cobelodus aculeatus, Akmonistion zangerli). Based on their description, however, the supposed ethmoidal articulation in Akmonistion probably corresponds to the orbital one in Cladodoides (although the extent to which the polar cartilage contributed to the basicranium in Akmonistion is admittedly uncertain). The posterior position of this articula- tion in Pucapampella, Debeerius, osteichthyans, and Acanthodes suggests that its more anterior position in elasmobranchs such as Cladodoides and Akmonistion is a derived condition. Paradoxically, the anterior position is apparently plesiomorphic for orbitostylic neoselachians (e.g., Chlamydoselachus) and it occupies a more posterior position in cladistically advanced orbitostylic taxa. In many orbitostylic sharks, the palatoquadrate lacks a "palatine" component; instead, the jaws are abbreviated and do not extend far (if at all) beneath the ethmoid region (e.g., Squalus, hexanchiforms). The posterior position of the orbital articulation may be a derived feature, conceivably related to the presence of a "shortened" palatine region.

Recent discoveries suggest that separate ethmoidal (or rostral) and palatobasal articulations are present in Debeerius (a primitive, nonholostylic paraselachian holocephalan; Grogan and Lund, 2000) and in Pucapampella (Maisey and Anderson, 2001). At present, it is not known whether a corresponding articulation is present in Gladbachus or Doliodus. In Pucapampella, an articular surface is present on the anteriomost part of the palatoquadrate on either side of a wide ethmoidal region as in osteichthyans (there is apparently no palatoquadrate symphysis; Maisey and Janvier, in prep.). Although the ethmoid region is still unknown in Pucapampella, the anterior palatoquadrate articulation evidently extended far anteriorly. Conjunction of the ethmoidal and palatobasal articulations in Pucapampella provides compelling evidence that both articulations were primitively present in chondrichthyans. As Schaeffer (1981: 57) noted, the palatoquadrate in modern chimaeroids is fused to the braincase adjacent to the polar cartilage, below the efferent pseudobranchial foramen (i.e., in the topographic position of the palatobasal articulation). Collectively, these findings support the hypothesis that ethmoidal and palatobasal articulations are primitively present in crown group gnathostomes and were conserved in primitive chondrichthyans. Since the palatobasal articulation was located in the posterior part of the orbit in primitive osteichthyans and chondrichthyans, its more anterior articulation in $\mathrm{Pa}-$ leozoic sharks such as Cladodoides is prob- 
ably apomorphic. Although the orbital articulation of modern orbitostylic sharks may be homologous to the osteichthyan palatobasal articulation, as claimed by Huxley (1876), De Beer (1931) and Gardiner (1984a), its unusual dorsal extent into the orbit is also probably apomorphic (Maisey, 1980). The ethmoidal articulation has presumably been lost secondarily in Cladodoides and modern orbitostylic sharks, but is conserved in Heterodontus and galeomorphs.

Trabeculae, Polar Cartilage, and Suborbital Shelf: According to De Beer (1931, 1937), after the polar cartilage becomes fused with the trabeculae in Scyliorhinus, its position is still marked by notches on the trabecular-polar bar. The notch defining its anterior margin contains the efferent pseudobranchial artery while the posterior one contains the pituitary vein, but these vessels become enclosed by separate foramina as chondrification becomes more advanced. In Etmopterus, the polar cartilage and trabeculae are united above and below the efferent pseudobranchial artery near the anterior margin of the polar cartilage (Holmgren, 1940). The efferent pseudobranchial and pituitary vein foramina therefore represent important developmental and topographic landmarks in neoselachians (and in gnathostomes generally) by indicating the original site and boundaries of the polar cartilage.

The bucco-hypophyseal fenestra (often secondarily closed in neoselachians) is located in front of the internal carotid opening, which represents an important topographic landmark indicating the extent of the polar cartilage contribution to the basicranium. During ontogeny, the hypophysis in lampreys and gnathostomes becomes associated with the ventral tip of the notochord within a sac or chamber (pituitary fossa or hypophyseal chamber) formed by an invagination of the cranial wall between the trabecularpolar cartilage and basal (parachordal) plate (Goodrich, 1930). In gnathostomes, there is usually an additional extracranial space (the subpituitary space of Allis, 1928) traversed by the pituitary vein and lying between the posterior ends of the trabecular-polar cartilages and the parachordals. In modern adult elasmobranchs, the bucco-hypophyseal fenestra is usually closed although a narrow passage leading to the hypophyseal chamber is sometimes present (e.g., Echinorhinus; Shirai, 1992: pl. 3B, 18A). The mode of development of the adenohypophysis in hagfish is still poorly understood.

In modern elasmobranchs, fusion between the trabeculae anteriorly produces an elongated median space (the polar fenestra; Holmgren, 1940). As development proceeds, this fenestra becomes reduced, leaving only the bucco-hypophyseal fenestra and internal carotid foramina. The ontogenetic timing of its closure is variable, occurring early in batoids and many sharks but later in Scyliorhinus. One of the earliest parts of the braincase floor to form is the postpituitary commissure, connecting the polar cartilages below the level of the parachordals and behind the internal carotids (e.g., Scyliorhinus; De Beer, 1931, 1937; Holmgren, 1940). This commissure separates the bucco-hypophyseal fenestra (the remaining portion of the polar fenestra) from the basicapsular fenestra in the parachordals. During early ontogenetic stages, the internal carotids also enter the braincase via the bucco-hypophyseal fenestra. A precarotid commissure subsequently develops, separating the carotid foramen from the remainder of the bucco-hypophyseal fenestra farther anteriorly. Just behind the carotid foramen, paired processes arise from the polar cartilages and extend inward toward the notochord, ultimately giving rise to the dorsum sellae.

According to Sewertzoff (1899), the optic and oculomotor foramina provide important developmental landmarks indicating the approximate line of fusion between the embryonic trabecular and orbital cartilages in the lateral walls of the braincase. Assuming that the relationship of the optic and oculomotor foramina to the trabeculae and orbital cartilage in Cladodoides was the same as in neoselachians, during ontogeny its trabeculae would have overlapped the polar cartilage posteriorly in the vicinity of the bucco-hypophyseal fenestra and dorsal to the anterior margin of the parachordal plate. In Chlamydoselachus, the orbital articulation fills the anterior part of the orbit (Allis, 1923a; Holmgren, 1940, 1941), although the locations of the efferent pseudobranchial and pituitary foramina suggest that the polar cartilage-de- 
rived area was no greater than in other neoselachians (unfortunately, the early developmental stages in Chlamydoselachus have never been described and the extent of its embryonic cranial cartilages is still conjectural).

The polar cartilage makes a relatively minor contribution to the braincase in modern osteichthyans (e.g., Amia; Bjerring, 1978) and it is reduced or even lost in some teleosts (where the pituitary vein passes ventral to the basicranium instead of entering it). It has been suggested that the polar cartilage made an extensive contribution to the basicranium in some arthrodires (e.g., Kujdanowiaspis, Tapinosteus; Stensiö, 1963a), based on the belief that the efferent pseudobranchial artery was located anteriorly and passed dorsal to the trabecular region before meeting the internal carotids, as in neoselachians. However, if it joined the internal carotid below the trabeculae and entered the braincase farther posteriorly (as in osteichthyans), such an interpretation is not justified (Young, 1980, 1986).

The polar cartilage apparently made a far greater contribution to the basicranium in Cladodoides than in neoselachians. There is also some evidence of extensive basicranial contribution by the polar cartilage in Tamiobatis vetustus and "Tamiobatis sp." In all these forms, the braincase has a generalized platybasic morphology and an elongated otic region (including the region formed by the parachordals). However, an extensive polar cartilage-derived region is also suspected in tropibasic Paleozoic sharks with a comparatively short otic region (e.g., "Cobelodus"; in prep.), based on the position of the efferent pseudobranchial foramen and the size of the hypophyseal chamber and dorsum sellae. On the other hand, in Orthacanthus the basicranial contribution of the polar cartilage was comparatively small, judging from the posterior position of the efferent pseudobranchial foramen within its orbit, although the braincase is platybasic and its otic region is elongated. The extent of polar cartilage and parachordal contributions to the basicranium therefore seems to have been considerably more variable in these Paleozoic sharks than in modern elasmobranchs.

In modern chimaeroids, the polar cartilage is almost as long as the trabecula (e.g., Callorhinchus; De Beer and Moy-Thomas, 1935), although it has a similar relationship to the efferent pseudobranchial artery and pituitary vein as in neoselachians (Stensiö, 1963a). It is uncertain whether the polar cartilage in chimaeroids is extensive from its first inception in the embryo or if it undergoes enlargement during ontogeny, but in Hydrolagus, the telencephalon is apparently elongated secondarily during ontogeny, and in earlier stages the brain is proportioned much as in modern sharks (G. Northcutt, personal commun. 2003). It is also uncertain whether presence of an extensive polar cartilage-derived region in the basicranium represents a synapomorphy of holocephalans and some Paleozoic sharks or a convergently acquired feature. A phylogenetic relationship between some "cladodont" sharks (especially symmoriids and stethacanthids) and holocephalans has been proposed elsewhere (e.g., Janvier, 1996: fig. 4.13.9; Coates and Sequeira, 2001a), although support for that proposal came mainly from equivocal postcranial features.

In Cladodoides, the suborbital shelf extends between the efferent pseudobranchial artery and pituitary vein, suggesting that it is developed on the lateral margin of the polar cartilage-derived region. In Orthacanthus, the suborbital shelf is much shorter and the efferent pseudobranchial foramen is located farther posteriorly (near the exit of the orbital artery; Maisey, 1983: fig. 13B), suggesting that its polar cartilage made a somewhat smaller contribution to the basicranium than in Cladodoides. According to De Beer (1937: 61), the suborbital shelf in 36-mm Scyliorhinus arises from two centers, one anterior (associated with the lamina orbitonasalis and forming a mesenchymatous connection with the palatoquadrate) and one posterior (enclosing the orbital artery). Holmgren (1940: 167-168) also described a similar arrangement of anterior and posterior suborbital mesenchymatous centers in 30-mm Scyliorhinus. In 40-mm embryos, he described the suborbital shelf as extending "along the trabecula, beginning in front with the cranioquadrate connection, from which its anterior part cannot be delimited." Behind this, the shelf continues along the trabecular margin, 
becoming thinner laterally and maintaining a membranous connection with the medial side of the palatoquadrate for most of its length. The efferent pseudobranchial artery accompanies "a posterior branch of the palatine nerve" through the suborbital shelf posteriorly, then crosses the floor of the orbit where it finally enters the braincase (Holmgren, 1940: fig. 118). This arrangement differs from that in Cladodoides, where the efferent pseudobranchial enters the braincase via a canal in the suborbital shelf, and only the ophthalmic artery passes through the orbital wall. However, both arrangements are similar in one important respect, namely the close relationship of the efferent pseudobranchial artery to the inferred extent of the polar cartilage, since in Scyliorhinus the vessel first passes through the suborbital shelf in the posterior part of the orbit, adjacent to the (very small) polar cartilage. If the efferent pseudobranchial artery was to become more completely enclosed by the suborbital shelf in Scyliorhinus, the vessel would not traverse the orbit before entering the braincase and would instead have a single entrance in the back of the orbit, in a position similar to Squalus.

Some correlation is noted in neoselachians between the presence and position of the orbital/palatobasal articulation and the extent of the suborbital shelf, which arises from anterior and posterior centers (De Beer, 1937; Holmgren, 1940). In modern orbitostylic sharks, there is no suborbital shelf anterior to this articulation, and a shelf is rarely developed farther posteriorly. One exception is Squatina, whose orbital articulation is oriented obliquely, with its lower part situated in the anterior part of the orbit (presumably the suborbital shelf meets the margin of the polar cartilage-derived region of the basicranium, although its ontogeny still has not been described). Where there is no orbital/palatobasal articulation, a suborbital shelf may be absent (e.g., batoids), or it may extend anteriorly from the site of the polar cartilage along the lateral margins of the trabeculae (e.g., Heterodontus and galeomorphs). In Cladodoides and many other Paleozoic sharks, the suborbital shelf is only found behind the articulation (resembling the situation in Squatina). The early chondrichthyan $\mathrm{Pu}$ - capampella presents us with yet another variation on this theme, since it has a broad suborbital shelf anterior to the palatobasal articulation. Such an arrangement is unknown in other elasmobranchs but is similar to osteichthyans, in which a shelf (usually much narrower than in Pucapampella) is present anterior to the palatobasal articulation and is covered ventrally by the parasphenoid (e.g., Amia, Moythomasia, Eusthenopteron, Holoptychius; Jarvik, 1980; Gardiner, 1984a). There is undoubtedly much homoplasy in all these variations, yet there may also be phylogenetically meaningful signals if the pattern in Pucapampella is primitive for chondrichthyans.

The relationship of the efferent pseudobranchial artery to the polar cartilage is consistent in modern sharks, but its relationship to the suborbital shelf is variable; in Squalus, the efferent pseudobranchial is located in the posterior part of the orbit (a suborbital shelf is absent), and the site of the polar cartilage is small; in Scyliorhinus, the artery first passes through the suborbital shelf before entering the cranial cavity at the trabecular-polar cartilage junction. In Cladodoides, the artery passed through cartilage forming the anterior part of the suborbital shelf (presumably near the anterior border of the polar cartilage), where it met the ophthalmic artery before entering the cranial cavity.

Beginning with Huxley (1875), several investigators have proposed that the trabecular cartilages are derived evolutionarily from the visceral arch skeleton. Allis (1923b) additionally suggested that the polar cartilage is of visceral arch origin. Three lines of evidence supported those early views (reviewed in De Beer, 1937: 375); the location of the subpituitary space (including the passage for the pituitary vein) below the dura mater and above the trabeculae, indicating that the latter are not part of the primitive cranial wall (Allis, 1923b); the position of the trabeculae anterior to the parachordal plate (and its associated somites), which precludes it from being derived from the axial skeleton; and experimental removal of neural crest tissue led to abnormal development of the trabeculae and visceral arches, indicating that their development was related. Both Allis (1923b) and De Beer (1937) suggested that the tra- 
beculae were formerly parts of a premandibular arch. Allis (1923b) also proposed that the polar cartilage represents a pharyngomandibular element, but De Beer (1937) objected because a supposed pharygomandibular has been identified between the palatoquadrate and braincase in some sharks (Sewertzoff and Disler, 1924; but see Holmgren, 1940, 1943; Gardiner, 1984a), and because the polar cartilage supposedly is of axial rather than visceral arch derivation. Holmgren (1943) claimed that certain dorsal elements of the prootic arches may have been incorporated into the braincase, Bertmar (1959) and Jarvik (1954) both believed that the ectomesenchymatic tissue of the trabeculae in fishes represent an infrapharyngomandibular, and Jarvik (1954) made elaborate reconstructions of the braincase in Eusthenopteron in which he identified parts of the premandibular and mandibular arches on the basis of topographic (serial) similarities.

Schaeffer (1981: 21) was highly skeptical of such scenarios, pointing out that "there is ... no evidence from studies of neural crest migration that elements of the visceral skeleton were literally incorporated into the chondrocranium" (see also Chibon, 1974; Schaeffer, 1975). Instead, Schaeffer (1981) argued that the apparent absence of visceral arch tissue in the side walls of actinopterygian and neoselachian braincases, which Bertmar (1959: 328, 338) had attributed to "modifications in early ontogeny", simply meant that ectomesenchymatic tissues are equally competent to form visceral or chondrocranial structures under certain conditions. In fact, it has now been shown that the rostralmost (trigeminal or mandibular) stream of neural crest cells (which ultimately populates the entire anterior head region), eventually condenses into a dorsal "maxillary" primordium below the eye, and a ventral "mandibular" primordium below the oral cavity and serially aligned with neural crest condensations of the visceral arches farther posteriorly (Francis-West et al., 1998; Chai et al., 2000; Meulemans and BonnerFraser, 2002). Somewhat surprisingly, the trabeculae develop from the dorsal primordium while the palatoquadrate and Meckel's cartilage both arise in the ventral condensa- tion (Cerny et al., 2004); no part of the jaws apparently forms in the supposed maxillary primordium. Thus, Schaeffer's (1981) prediction regarding ectomesenchymatic competence seems to be met (at least as far as the trabeculae are concerned), although it is still uncertain what the "certain conditions" he advocated might be; one possible factor is the absence of Hox (homeobox transcription factor) expression in the trigeminal neural crest stream (Hox is expressed in the hyoid/ preotic and common branchial/postotic streams farther posteriorly); this has already been implicated in the origin of jaws (Cohn, 2002) and may also have played a role in the evolution of the trabecular plate in gnathostomes. Mandibular and premandibular regions of the trigeminal neural crest stream have not so far been recognized, although the dorsal "maxillary" condensation extends into the premandibular region anteriorly. Moreover, Dlx 1 cognates in the lamprey are expressed throughout the mandibular and premandibular regions, but only in the mandibular region of gnathostomes (Kuratani et al., 2004). Thus, the absence of Hox expression in the entire prechordal region, and the restricted expression of Dlx cognates (and related ectodermally derived growth factors) in mandibular ectomesenchyme, may be viewed as developmental synapomorphies that are linked to the evolution of trabeculae and jaws in gnathostomes.

Optic Pedicel AND Position OF OCulOMOTOR FORAMEN: An optic pedicel is absent in chimaeroids and in all modern osteichthyans (with the possible exception of Acipenser; Gardiner 1984b). Gardiner's (1984a, 1984b) suggestion that the pedicel represents a primitive gnathostome character is supported by recent discoveries of an eyestalk attachment in early osteichthyans (e.g., Ligulalepis, Psarolepis; Basden et al., 2000; Zhu et al., 2001). There is also evidence of a similar attachment in many placoderms associated with foramina for the optic and oculomotor nerves, ophthalmic artery, and pituitary vein as well as attachments for various extrinsic eye muscles (Young, 1986). The pedicel is positioned behind the optic foramen and beneath the oculomotor foramen in both elasmobranchs and placoderms. Young (1986) concluded that the similarity could be 
interpreted either as a synapomorphy of these groups or as a primitive gnathostome condition. In Ligulalepis, the attachment area lies above and behind the optic foramen and below and anterior to the oculomotor foramen (Basden et al., 2000), but in Psarolepis the attachment area is behind the optic foramen as in elasmobranchs and placoderms (Zhu et al., 2001), suggesting that it is a conserved gnathostome pattern and not a synapomorphy of placoderms and elasmobranchs. Having the oculomotor foramen situated slightly farther posteriorly in Ligulalepis may be autapomorphic, although its phylogenetic significance cannot be reliably determined given the absence of a pedicel in other primitive actinopterygians. According to $\mathrm{Yu}$ (1998: fig. 1C), in Psarolepis the oculomotor nerve is located above a large pit (said to be for the pituitary vein); however, according to the illustration of Psarolepis in Zhu et al. (2001: fig. 1d), this pit forms the pedicel attachment area, in which case the oculomotor foramen is dorsal to the attachment as in elasmobranchs and placoderms.

TRIGEMinal, FACIAL, AND OCtAVOLATERAL NERVES: The arrangement of the trigeminal and facial nerves is often difficult to reconstruct in fossils, as exemplified by the differing interpretations of Cladodoides and Tamiobatis in Gross (1937), Romer (1964), Schaeffer (1981), and earlier in the present work. In neoselachians, the ventral part of the lateral commissure is rarely chondrified. In addition, very few soft structures pass through the postorbital process of neoselachians, and consequently any interpretation of their arrangement in Cladodoides or other Paleozoic sharks lacks a reliable modern paradigm. It is nevertheless possible to make comparisons that are at least congruent with the general arrangement of major nerves and vessels in the postorbital region of neoselachians, even though their relationship to the process may differ. The interpretation of nerves in Cladodoides presented earlier agrees in most part with the arrangements seen in neoselachians, but certain features (especially the arrangement and topographic position of the major nerve ganglia) vary in modern forms and do not provide a satisfactory paradigm for interpreting fossils. Furthermore, treating the octavolateral nerves as separate entities (Northcutt and Bemis, 1993) has added an additional layer of complexity to the identification of cranial nerve foramina in fossils.

Excluding the ophthalmic profundal branch $\left(\mathrm{V}_{1}\right)$, the gnathostome trigeminal nerve includes a maxillary ramus $\left(\mathrm{V}_{2}\right.$, sometimes regarded as a pretrematic ramus, although its equivalence to a pretrematic branchial ramus is conjectural) and a mandibular ramus $\left(\mathrm{V}_{3}\right.$, sometimes regarded as a posttrematic branch). In gnathostomes generally, the trigeminal nerve also has a dorsal superficial ophthalmic ramus (absent in Latimeria; Northcutt and Bemis, 1993). In neoselachians, this ramus can be separate (e.g., Squal$u s$; Norris and Hughes, 1920) or intimately associated with the superficial ophthalmic ramus of the anterodorsal lateral line nerve, forming the superficial ophthalmic complex (e.g., Chlamydoselachus; Allis, 1923a). The ganglia of the trigeminal nerve and buccal ramus of the anterodorsal lateral line nerve can be so closely associated as to be inseparable.

In Chlamydoselachus, the maxillary ramus of the trigeminal nerve divides into three principal ramules, a posterior one (extending to the superior labial levator muscle and the ventral edge of the palatoquadrate) plus two anterior ones that pass across the ectethmoid process and extend ventral to the nasal opening (Luther, 1909; Allis, 1923a). As in many other neoselachians, most of the maxillary ramus in Chlamydoselachus is fused with the buccal ramus of the anterodorsal lateral line nerve (formerly considered part of the facial nerve; see below), an arrangement which may be primitive for gnathostomes (Northcutt and Bemis, 1993). The mandibular ramus in Chlamydoselachus runs outward across the anterodorsal margin and outer surface of the superior maxillary levator muscle, then across the mandibular adductor muscle. It typically supplies the dorsal mucosa as well as connective tissues of the hyoid arch and the floor of the mouth and therefore extends much farther laterally than the other branches of the trigeminal nerve. According to Allis (1923a: 212), "in the dorsal part of its course it first sends a branch posteriorly beneath the postorbital process to the outer surface of the levator maxillae superioris". 
In Squalus, the mandibular ramus also extends laterally in front of the postorbital process (Norris and Hughes, 1920; Marinelli and Strenger, 1959).

In many neoselachians, the trigeminal foramen lies either in the orbital opening of the jugular canal (e.g., Oxynotus, Scymnodon) or in front of it within the posterior part of the orbit (e.g., Etmopterus, Squatina, Rhinobatus). However, in some taxa the foramen lies much farther posteriorly, behind the postorbital process (e.g., Carcharodon; Parker, 1887).

The facial nerve of gnathostomes includes three principal rami after the sensory lateralis components are removed from consideration: the palatine ramus (equivalent to the pharyngeal branch of a typical branchial nerve), the mandibular ramus (equivalent to the pretrematic branch?; note however that such homologies with branchial nerves are speculative), and the hyoid ramus (the posttrematic equivalent?). The mandibular and hyoidean rami arise from the main (hyomandibular) trunk, from which the palatine ramus first branches at the geniculate ganglion. The palatine ramus typically passes anteroventrally to supply the dorsal oral epithelium, the internal mandibular ramus supplies the internal surface of the lower jaw, and the hyoidean ramus supplies the branchial muscles of the hyoid arch (Norris and Hughes, 1920).

In Squalus and many other squaloids, the palatine nerve arises intracranially from the hyomandibular trunk and then penetrates the basal part of the lateral commissure. However, in other neoselachians the palatine ramus often arises extracranially and there is no separate palatine foramen (e.g., batoids, Chlamydoselachus and hexanchiforms, Squatina, galeomorphs, and Heterodontus). Holmgren (1941) observed that the palatine nerve is entirely extracranial in embryos of Squalus and Etmopterus, although it becomes enclosed proximally by cartilage later in ontogeny. Upon emerging from the braincase, the palatine ramus in Squalus divides into an anterior and a posterior ramule (Norris and Hughes, 1920). Its anterior division passes anteriorly between the cranium and the palatoquadrate, while the posterior branch (sometimes termed the pretrematic ramus, but again any equivalence to the pre- trematic ramus of a branchial nerve is speculative) passes posteriorly at the mesial ventral border of the palatoquadrate. It then turns ventrally, where it meets the spiracle and runs along the dorsolateral surface of Meckel's cartilage. This subdivision of the palatine ramus and the initial separation of the mandibular and hyoidean rami are always extracranial in neoselachians.

The hyomandibular trunk leaves the braincase behind the postorbital process in many neoselachians (e.g., Scymnodon, Oxynotus; Holmgren, 1941). Gardiner (1984a: 239) regarded this as a derived condition, possibly associated with the posterior position of the hyomandibular fossa in elasmobranchs (in contrast with osteichthyans, in which the fossa is located farther anteriorly). The mandibular ramus of this trunk often initially accompanies the maxillary ramus of the trigeminal. These may cross the floor of the orbit together before the mandibular ramus angles sharply backward (e.g., Laemargus), or the mandibular ramus may separate sooner and cross the posterior wall of the orbit to reach the posterior angle of the mouth (e.g., Chlamydoselachus, Squalus; Daniel, 1934).

Discussion of the trigeminal and facial nerves in Paleozoic sharks necessarily involves consideration of the transverse passage through the postorbital process described earlier. Gross (1937) did not identify any opening from this passage within the orbital surface of the process, but Romer (1964) found such an opening in Tamiobatis vetustus (which he interpreted as containing the pretrematic or mandibular ramus of the facial nerve). This led Schaeffer (1981) to suggest that such an opening may also be present but not identified in Cladodoides, which is now confirmed by the CT scan. Schaeffer (1981) considered that the canal contained a laterally situated palatine ramus of the facial nerve in Tamiobatis, Orthacanthus, and Cladodoides. Coates and Sequeira (1998) proposed that the palatine ramus also penetrated the postorbital process in Akmonistion; furthermore, they found evidence for two canals in one of their specimens and suggested that at least one was big enough to have carried both nerves and blood vessels (perhaps including a branch of the orbital artery and a palatine ramule). Thus, several al- 
ternative interpretations have been advanced regarding the function of this canal in Paleozoic sharks.

Romer's (1964) proposal that the mandibular ramus of the facial nerve passed through the postorbital process in Tamiobatis vetustus (or Cladodoides) is considered implausible. In Chlamydoselachus, this ramus is directed posterolaterally rather than laterally and extends far behind the postorbital process to supply the medial surface of the lower jaw (as in gnathostomes generally; Luther, 1909; Allis, 1923a). Even in Squalus (which has short, transverse jaws), the facial mandibular ramus is directed posterolaterally away from the postorbital process (Norris and Hughes, 1920; Marinelli and Strenger, 1959). Indeed, if the mandibular ramus passed through the postorbital process as Romer (1964) proposed, it would emerge on the wrong (i.e., lateral) surface of the mandibular arch. Additionally, this ramus is normally associated with the spiracular opening behind the postorbital process (Dick, 1978).

Several criticisms can be leveled at Schaeffer's (1981) proposal that the palatine ramus passed laterally through the postorbital process. First, in neoselachians the ramus passes ventrally toward the roof of the oral cavity and does not extend very far laterally. Therefore, while its course may carry it through the unchondrified floor of the lateral commissure (e.g., Squalus, Etmopterus), the ramus never enters the postorbital process farther laterally. Second, the course of the palatine ramus in Cladodoides (including its distal ramules) is clearly established and agrees essentially with the arrangement in neoselachians. It penetrated the chondrified floor of the postorbital process medial to the jugular canal and emerged beneath the braincase without passing laterally. Since this nerve supplies the roof of the oral cavity in gnathostomes, it is unlikely that any additional palatine ramules passed farther laterally through the postorbital process. Third, the main facial (hyomandibular) foramen is not aligned with the transverse canal in the postorbital process. Finally, scanning shows that the supposed foramen for the palatine nerve at the distal end of the postorbital process in Cladodoides shown by Schaeffer (1981: fig. 7C) is merely a minor branch and not the main passage. It is therefore considered unlikely that the palatine nerve traversed the postorbital process laterally in Cladodoides or other Paleozoic sharks.

Coates and Sequeira (1998: 79) commented that presence of distal foramina in the postorbital process is "a likely primitive chondrichthyan synapomorphy", implying homology across a wide suite of taxa. In fact, if such foramina were for the trigeminal mandibular ramus or for the buccal-maxillary complex, their position may conceivably reflect a more fundamental (e.g., gnathostome?) pattern of cranial nerves. However, the arrangement of the trigeminal nerve inferred here in Cladodoides apparently differs from that in Acanthodes, where the trigeminal mandibular ramus lay behind the postorbital process and passed through the palatoquadrate (Miles, 1973; see below). In Pucapampella, there is no evidence of a canal in the lateral wall of the postorbital arcade as in Cladodoides. It is possible that the peculiar arrangement found in Cladodoides and Tamiobatis vetustus is an apomorphic character, but further analysis is necessary.

The octavolateral nerves in gnathostomes (sensu Northcutt and Bemis, 1993) include the octaval (acousticovestibular) nerve plus all the lateralis components classically considered to be parts of the facial, glossopharyngeal, and vagal nerves. Two classical lateralis components of the facial nerve (the superficial ophthalmic and buccal branches) form the anterodorsal lateral line nerve, whose main trunk usually accompanies the trigeminal through the prootic foramen. During ontogeny, the trigeminal and anterodorsal lateral line nerves frequently become separated from the facial nerve by the prefacial commissure (discussed below). The superficial ophthalmic components of the anterodorsal lateral line and trigeminal nerves may combine, forming the superficial ophthalmic complex. The buccal ramus is often fused with the maxillary ramus of the trigeminal to form the buccal + maxillary complex. In Chlamydoselachus, the supraorbital sensory canal is innervated by ramules of the superficial ophthalmic ramus passing through the supraorbital cartilage, while the posterior part of the infraorbital canal is supplied by ramules from the buccal ramus (some of which 
pass posterodorsally through the roof of the postorbital process; Allis, 1923a: 197). The anteroventral lateral line nerve (classically, the external mandibular ramus of the facial nerve) is closely associated (or even fascicularized) with the hyomandibular trunk, and these nerves may leave the braincase together.

Additionally, there is an otic lateral line nerve (classically the otic ramus of the facial nerve) supplying the posterior continuation of the supraorbital canal and the spiracular organ (where present) plus three postotic lateral line nerves (middle, supratemporal, and posterior). In Chlamydoselachus, several ramules of the otic lateral line nerve pass through the postorbital process and there is a large canal opening onto the roof of the braincase medial to the spiracle (Allis, 1923a). In Squatina, there is large opening in the orbital roof which was said to transmit the otic ramus by Gegenbaur (1872), but this is mainly occupied by the orbital process of the palatoquadrate (Iselstöger, 1937). The middle lateral line nerve accompanies the glossopharyngeal nerve, while the supratemporal and posterior lateral line nerves accompany the vagal nerve. Among neoselachians, some (perhaps all) batoids lack any glossopharyngeal lateralis component (Norris and Hughes, 1920), but in Squalus a minute nerve arises from a small lateral line ganglion at the root of the glossopharyngeal nerve and passes through a short canal in the lateral wall of the otic capsule to the dorsal side of the head (the ramus supratemporalis IX of Norris and Hughes, 1920). Also in Squalus, the supratemporal nerve (their ramus supratemporalis $\mathrm{X}$ ) leaves the vagal canal and passes through the posterior wall of the otic capsule, but the posterior nerve (their ramus dorsalis X) does not pass through any cartilage. Allis (1923a) did not describe the arrangement of these nerves in Chlamydoselachus, but he noted that parts of the supratemporal and anterior lateral line canals are innervated by branches extending from the glossopharyngeal and vagal nerves. From his illustrations, it is likely that the middle and supratemporal lateral line nerves pass through cartilage forming the lateral and posterior walls of the otic capsule, as in Squalus. The passages for two otic lateral line nerves have been traced in CT scans of Notorynchus (Maisey, 2004b), where a small dorsal canal (probably for the middle lateral line nerve) branches from the glossopharyngeal canal and passes dorsolaterally through the otic capsule into the saccular region. Another small canal (probably for the supratemporal lateral line nerve) branches from the vagal canal and passes through the posterior wall of the otic capsule, just behind the posterior semicircular canal.

TRIGEMINO-FACIAL RECESS: In craniates generally, the trigeminal and facial ganglia become enclosed by a chamber formed in the membranous lateral wall of the cranium during ontogeny. Allis (1928) envisioned that "if the mesial wall of this chamber were to be broken down, the chamber would become a trigemino-facialis recess, while if this wall persisted and the lateral wall were broken down, the trigeminal and facialis ganglia would lie on the external surface of the primitive skull." Allis (1914, 1923a) had already described what he termed the acustico-trigemino-facialis recess in Chlamydoselachus, an internal feature located immediately anterior to the otic capsule within the lateral wall of the cranial cavity and containing the roots of the trigeminal, facial, and octaval nerves. He also described the trigemino-pituitary fossa, an external depression in the posterior wall of the orbit which contains a foramen for the pituitary vein and another for the trigeminal and abducent nerves. The trigeminal ganglion lies in this fossa (which is closed externally by connective tissue surrounding the lateral head vein). The external rectus muscle (supplied by the abducent nerve) also arises in this fossa, just behind the pedicel. Thus, in Chlamydoselachus the trigemino-pituitary fossa is an important landmark that contains the ganglia of the trigeminal and facial nerves, the abducent nerve, the pituitary vein, and the origin of the external rectus muscle.

In neoselachian embryos, the trigeminopituitary fossa develops at the junction of the parachordal and the trabecular-polar cartilages, with the pituitary vein sandwiched between them inside an extracranial space formed at the posterior end of the polar cartilages (Allis, 1928; De Beer, 1931, 1937). The embryonic prootic foramen, acustico-tri- 
gemino-facial recess, prefacial commissure, and lateral commissure are all located immediately behind this region. This may also have been the case in hybodonts and Orthacanthus, but not in Cladodoides (see above). It is concluded that presence of a trigeminopituitary fossa (sensu Allis, 1914) is not a synapomorphy of elasmobranchs generally, but may be a synapomorphy of a group comprising neoselachians and some extinct elasmobranchs.

In neoselachians, the medial wall of the otic capsule becomes secondarily fused to the parachordal plate in the vicinity of the prefacial commissure (De Beer, 1937; Holmgren, 1940). The acustico-trigemino-facial recess (an internal space, sensu Allis, 1914) forms mainly from the medial capsular wall, although it is contiguous with the prefacial commissure (which separates the facial nerve proper from the trigeminal and anterodorsal lateral line nerve trunks). This recess lies behind the trigeminal and facial ganglia and also contains the octaval (acousticovestibular) nerve. In Cladodoides, the hyomandibular and trigeminal foramina are separated by the prefacial commissure, which connects the floor of the anterior ampulla to the parachordal cartilage and forms a wall behind the trigeminal and facial ganglia (like the inner face of the acustico-trigemino-facial recess in neoselachians). In Chlamydoselachus and hexanchiforms, the hyomandibular trunk emerges just below and slightly behind the postorbital process (the postorbital process only chondrifies above the lateral head vein and does not form a complete jugular canal; Allis, 1923a; Maisey, 2004b). In many adult squaloids, the facial (hyomandibular) foramen is located even farther posteriorly (on the lateral wall of the otic capsule; Holmgren, 1941). In Heterodontus, the foramen lies in the posterior part of the orbit, and in many galeomorphs the hyomandibular trunk and trigeminal nerve emerge together (Goodrich, 1930; Holmgren, 1940, 1941).

Schaeffer (1971) suggested that in actinopterygians the term "trigemino facialis chamber" should be restricted to the extramural cavity between the lateral cranial wall and the region formed by the lateral commissure. Gardiner (1984a: 236) further concluded that the chamber in primitive actin- opterygians "is not very different from that of selachians such as Oxynotus, Squatina and Squalus." The presence of a trigemino-facial chamber in some placoderms (e.g., Brindabellaspis; Young, 1980) suggested to Gardiner (1984a) that it represents a synapomorphy of gnathostomes generally. However, in arthrodires the facial nerve passes through the anterior postorbital process and does not lie within a trigemino-facial chamber as in osteichthyans and neoselachians. It is unclear whether this pattern is primitive for gnathostomes or apomorphic for arthrodires.

In Pucapampella, a large space extends posteriorly from a narrow prefacial commissure, passing medial to the postorbital process and the jugular canal. The space extends posterior to the postorbital process and below the anterior ampulla (Maisey, 2001c: fig. 16.1). I originally termed this space a trigemino-facial recess, but it probably contained the profundal, trigeminal, abducent, and facial nerves (including the hyomandibular trunk) and perhaps even the pituitary vein. In fact, the space clearly extends behind the confines of the orbit (as defined by the postorbital process) and is almost as extensive as the embryonic prootic foramen of modern gnathostomes. The optic and prootic foramina in Pucapampella are separated by a narrow bar of cartilage (corresponding to the pila antotica, although it was originally misidentified as the prefacial commissure). The condition in Pucapampella supports the suggestion that cladistically primitive elasmobranchs lacked a trigemino-facial chamber as in neoselachians.

Postorbital Process: The morphology of the postorbital process in most neoselachians is considerably simpler than in Cladodoides. Even in neoselachians, however, the ontogeny of the process may involve tissues from different parts of the head skeleton. According to Holmgren (1940: 54, fig. 55), the first part of the process to develop (in 38-39-mm Squalus embryos) is a small blastemic element located just in front of the prootic foramen, which he termed the primary postorbital process. In later (48-49-mm) embryos, this element is connected to the blastemic supraorbital crest and these chondrify together to form what he termed the secondary postorbital process (in Scyliorhinus, however, 
his "primary" postorbital process develops directly from the supraorbital blastema, blurring its distinction from the secondary process). At the same time, a laterally directed shelf is formed on the ventral part of the otic capsule below the main (hyomandibular) facial foramen and behind both the palatine ramus and the orbital artery. In Squatina and some squaloids, a connective tissue lamella (which does not invariably become chondrified) joins this shelf to the primary postorbital process, covering the hyomandibular trunk and lateral head vein laterally. There is general consensus that this lamella is homologous to the lateral commissure in osteichthyans, which is also lateral to the jugular canal and forms the sidewall of the trigeminofacial chamber (De Beer, 1937; Holmgren, 1940; Schaeffer, 1981; Gardiner, 1984a).

The extent to which the postorbital process becomes chondrified in neoselachians is highly variable. There may be a well-developed process behind the orbit, even in forms in which the lateral commissure is said to be absent (or at least does not persist into the adult; e.g., hexanchiforms, galeomorphs, many batoids; Holmgren, 1941). Only rarely is the lateral commissure fully chondrified, forming a complete arch enclosing the jugular canal and connected both dorsally and ventrally to the braincase (e.g., Squatina, Centrophorus). In many neoselachians, there is no postorbital cartilage lateral or ventral to the lateral head vein, only above it.

As described by Holmgren (1940), the adult postorbital process in Squalus, Etmopterus, and Scyliorhinus consists only of the primary process and does not include the lateral commissure, which remains membranous and unchondrified. In some galeomorphs (e.g., Carcharhinus), a supraorbital cartilage is absent but a postorbital process is still present on the sidewall of the otic capsule (although the process is often poorly chondrified). These observations suggest that, while the primary postorbital process (formed from blastemic tissue primitively associated with the supraorbital shelf) is often chondrified in neoselachians, the lateral commissure and the ventral shelf arising from the otic capsule only rarely become chondrified. Presumably, the developmental mechanism leading to chondrification of the lateral commissure in primitive extinct sharks and in neoselachians such as Squatina and Centrophorus is suppressed or absent in most neoselachians. Chondrification patterns in the neoselachian postorbital process thus include (1) lateral commissure is present and all parts of the postorbital process (including the primary process and the ventral shelf) are fully chondrified (e.g., Squatina, Centrophorus); (2) lateral commissure and ventral shelf are present but unchondrified; the (primary) postorbital process is chondrified only above the lateral head vein (e.g., Squalus, Etmopterus, Rhinobatus); (3) lateral commissure and ventral shelf are absent, (primary) postorbital process is chondrified only above the lateral head vein (e.g., Carcharhinus, Mustelus); and (4) postorbital process is absent, with no evidence of a primary postorbital process, ventral shelf, or lateral commissure (e.g., Heterodontus, Orectolobus, Raja, Torpedo, Discobatus).

The postorbital process in Cladodoides is presumed to include cartilage derived from the lateral commissure, primary postorbital process, and lateral otic shelf. There is no evidence to suggest that the lateral commissure fused farther ventrally with the parachordal plate as in osteichthyans, and the cartilage behind the palatine ramus and below the hyomandibular foramen in Cladodoides corresponds closely to the lateral shelf arising from the otic capsule in Squalus as described by Holmgren (1940). The orbital artery and palatine ramus in Cladodoides (including its anterior and posterior ramules) apparently passed through the lateral commissure and were subsequently enclosed in the floor of the process medial and ventral to the jugular canal as chondrification progressed.

The position of the postorbital process on the lateral surface of the braincase is highly constrained during ontogeny. Nevertheless, some variation is noted in the position of the process relative to other features of the braincase in modern and fossil elasmobranchs. The postorbital processes lies entirely anterior to the labyrinth region in Cladodoides, in Orthacanthus and the "Tamiobatis sp." braincase described by Schaeffer (1981: figs. 14, 22, 23), and in chimaeroids and Puca- 
pampella (Maisey, 2001c). By contrast, in many neoselachians the postorbital process is lateral to the anterior ampulla, and in some carcharhiniforms the ampulla is positioned even farther anteriorly. The anterior ampulla also extends in front of the postorbital process in hybodonts (e.g., Tribodus, Hybodus). The anterior ampulla is situated between the anterior postorbital process in placoderms such as Kujdanowiaspis (Stensiö, 1969).

According to De Beer (1937) and Holmgren (1940), the medial wall of the jugular canal in Squalus is formed by late chondrification of a connective tissue bridge between the lateral capsular wall and the suborbital cartilage. The position of this bridge relative to the labyrinth probably determines the location and extent of the inner wall of the jugular canal, and little topological variation relative to the otic capsule and parachordal plate is therefore possible. However, the location at which the lateral commissure is fused to the braincase dorsally (the primary postorbital process sensu Holmgren, 1940) is determined by the posterior extent of the supraorbital cartilage, which is potentially more variable. In Cladodoides, the entire postorbital arcade is inclined obliquely in lateral view, with its dorsal part extending farther anteriorly than its ventral part. A more posteriorly located dorsal attachment would impart a more vertical orientation to the arcade behind the trigeminal and facial foramina, which would then lie either within the jugular canal or even within the orbit (fig. 32 ). Thus, the seemingly profound topographic differences noted earlier in the configuration of these nerves may result from differing orientations of the lateral commissure during ontogeny, possibly resulting from variation in the anteroposterior position at which it is fused to the primary postorbital process arising from the supraorbital cartilage. Given the general morphological agreement between the trigemino-facial chamber in modern elasmobranchs and early actinopterygians noted by Gardiner (1984a), the postorbital position of the facial foramen, the inclination of the postorbital process, and the upturned jugular canal may be apomorphic characters shared by Cladodoides, Tamiobatis vetustus, and Akmonistion.

The postorbital process in neoselachians

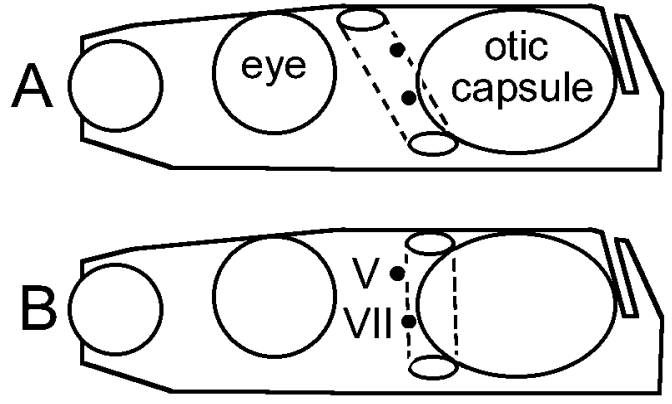

Fig. 32. Diagrammatic representation of postulated topographic differences in the dorsal attachment point of the lateral commissure in (A) Cladodoides and (B) a generalized hybodont or neoselachian. The positions of the trigeminal and facial nerves relative to the otic capsule and eye are identical, but they lie behind the orbit in panel $\mathrm{A}$ and within it in panel $\mathrm{B}$. This probably reflects differences in the anteroposterior extent of the supraorbital shelf (including the primary postorbital process, to which the lateral commissure is secondarily fused). In many neoselachians, the lateral commissure is unchondrified or absent, but the primary postorbital process is commonly present. Anterior to left. No scale.

and osteichthyans certainly develops in broadly similar fashion, with dorsal and ventral projections from the braincase connected by the lateral commissure. However, according to De Beer (1937: 391) the embryonic derivations of the dorsal and ventral processes differ; the dorsal process in osteichthyans is formed by the prootic process (a lateral projection from the wall of the otic capsule) rather than from an independent blastemic element as in neoselachians; and the ventral (basitrabecular) process in osteichthyans (and the postpalatine process farther ventrally) is derived from the margins of the trabeculae and parachordal plate, respectively, not from the ventral part of the otic capsule as in neoselachians. Thus, while the lateral commissure of neoselachians is probably homologous to that of osteichthyans, the adult postorbital process is not identical in these groups because its dorsal and ventral attachments to the lateral wall of the braincase involve tissues of different embryonic origins.

The postorbital process in Acanthodes forms the lateral part of a large dorsal ossification and has been described in some detail (Watson, 1937; Miles, 1965, 1973; Jar- 


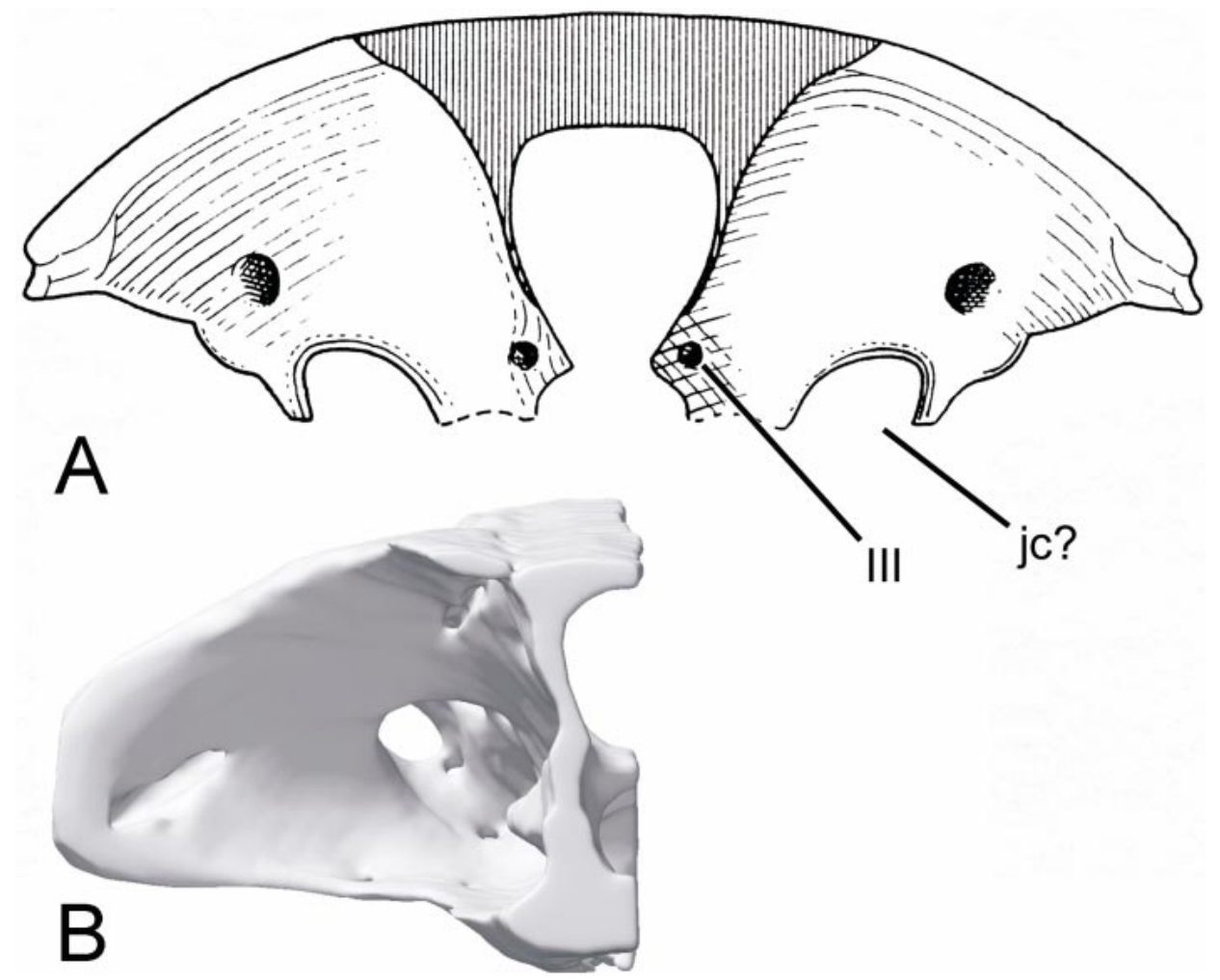

Fig. 33. Comparison of the postorbital process in anterior view: (A) Acanthodes (after Miles, 1973); (B) Cladodoides. See text for discussion. Not to scale.

vik, 1977, 1980). Unfortunately, certain aspects of its morphology are controversial and a reinvestigation in Acanthodes is necessary before some of the inconsistencies noted here can be resolved. The postorbital process in Cladodoides resembles that of Acanthodes although there are important diferences; for example, the postorbital process in Cladodoides probably contained the mandibular ramus of the trigeminal nerve (see above), whereas there is general agreement that in Acanthodes this ramus passed through the otic process of the palatoquadrate farther posteriorly.

The orbital surface of the process in Acanthodes contains a canal. Watson (1937) identified it a jugular canal, Holmgren (1942: 137) regarded it as a canal for the otic lateral line nerve ("otical part of the lateral sensory line”), Miles (1973) suggested it may have housed the middle cerebral vein, and Jarvik (1977) considered that it contained a supraorbital vein connected to the lateral head vein farther ventrally. In Acanthodes, the ventral margin of the postorbital process contains a wide embayment. According to Jarvik (1977) this contained the trigeminal nerve, but according to Miles (1973) it represents part of a trigeminofacial chamber. There is general agreement that another foramen located farther medially and anteriorly is for the oculomotor nerve. Significantly, no jugular canal was recognized either by Holmgren (1942) or Miles (1973).

Apart from the postorbital articulation (discussed below), these features are virtually the only landmarks of the postorbital process that can be compared in Acanthodes and Cladodoides. Simply from a topographic viewpoint, the ventral embayment in Acanthodes superficially resembles the dorsal margin of the jugular canal in Cladodoides (fig. 33). Unfortunately, the course of the lateral head vein in Acanthodes has only been reliably determined farther posteriorly, where it lay in a shallow groove on the lateral surface of 
the otic region, dorsal to the glossopharyngeal foramen and angled toward the embayment (Miles, 1973: fig. 2; Jarvik, 1980: fig. 260). The ventral embayment in Acanthodes was identified by Miles (1973) as the dorsal part of a trigemino-facial recess, but it could conceivably have contained the lateral head vein if this had the same relationship to the embryonic lateral commissure as in modern elasmobranchs (and as inferred in Cladodoides). In fact, since Miles (1973: fig. 4) considered the lateral wall of the embayment to be formed from the lateral commissure, it is difficult to envisage where else the lateral head vein might have passed (except perhaps through the small canal farther dosolaterally, which Miles regarded as possibly for the middle head vein). Miles' (1973) interpretation implies that the lateral head vein lay much farther ventrally, and/or that the lateral commissure extended much farther dorsally (relative to the lateral head vein) than in modern gnathostomes. However, if either the large ventral embayment or the smaller foramen farther dorsolaterally in Acanthodes contained the jugular canal, the process would agree morphologically with that of modern gnathostomes in having the lateral head vein enclosed by the lateral commissure. The position of the embayment relative to the trochlear foramen is relatively uninformative, since it lies at the same horizontal level as the trigeminal foramen and the dorsal margin of the jugular canal in Cladodoides, and at the same level as the ventral embayment in Acanthodes. The ossification supporting the main otic condyle in Acanthodes is probably homologous to the sphenotic of osteichthyans (Gardiner, 1984a). Only the "auxiliary otic condyle" (Miles, 1973) is located on the postorbital process farther anteriorly. Its position provides circumstantial support for reinterpreting the ventral embayment as the roof of a jugular canal because it is apparently the only part of the postorbital articulation that conceivably formed on the lateral commissure.

In arthrodires, the anterior postorbital process encloses the jugular canal and is located on the lateral wall of the otic capsule, slightly anterior to the anterior ampulla (e.g., Dicksonosteus; fig. 34). The arthrodire anterior postorbital process was therefore probably

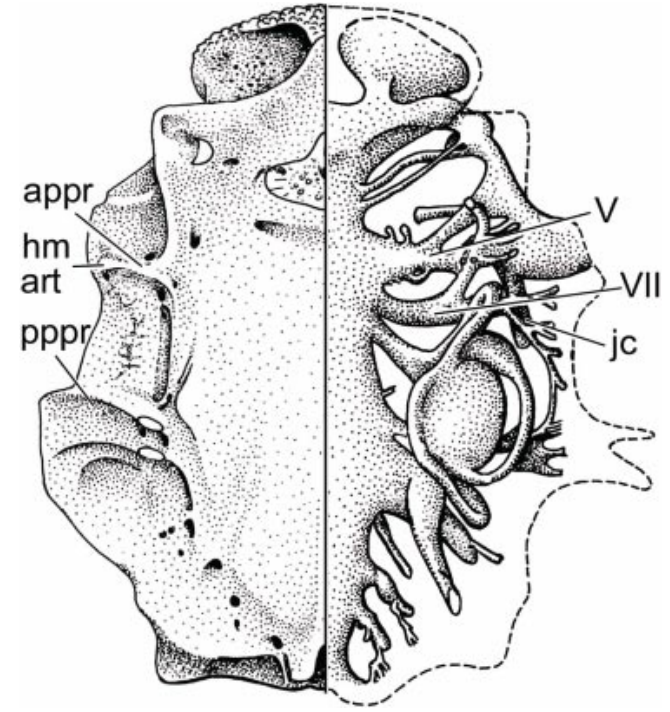

Fig. 34. Braincase of the arthrodire Dicksonosteus (illustrations derived from Goujet, 1984: figs. 6 and 26, and combined here). The left side shows the braincase in ventral view, and the right side shows the endocast in dorsal view. The anterior postorbital process bears the hyomandibular fossa and surrounds the jugular canal and facial nerve. No scale.

derived ontogenetically from the embryonic lateral commissure, like the postorbital process in crown-group gnathostomes (Stensiö, 1963a). However, the arthrodire anterior postorbital process differs from the elasmobranch postorbital process in at least two important respects: (1) the anterior postorbital process bears a hyomandibular fossa, unlike in elasmobranchs; (2) the anterior postorbital process contained the hyomandibular trunk of the facial nerve and is located far behind the main trigeminal foramen (which opens into the medial side of a jugular groove or pit). The prefacial commissure is positioned medial to the lateral commissure in elasmobranchs, but is presumably posterior to it in arthrodires. Thus, even if the placoderm anterior postorbital process is derived from the lateral commissure, its relationships to surrounding structures would have been different from those found in modern osteichthyans and chondrichthyans.

Several investigators considered that the lateral commissure is of neurocranial origin (Swinnerton, 1902; De Beer, 1937; Hammar- 
berg, 1937; Hubendick, 1943; Daget and d'Aubenton, 1957; Bertmar, 1959, Schaeffer, 1981), but others have suggested a visceral arch origin (e.g., in actinopterygians; Holmgren, 1943, and in sharks; Jollie, 1971; see discussion in Gardiner, 1984a). There has been little modern developmental work on this part of the braincase, but according to Bertmar (1959) the lateral commissure in Neoceratodus is derived from the hyoid arch (which suggests it may have formed in the hyoid/preotic stream of neural crest tissue). On the other hand, Holmgren (1940, 1943) considered that the elasmobranch lateral commissure arose in the mandibular arch (which suggests that it originated in the trigeminal stream). If the lateral commissures in Neoceratodus and elasmobranchs arise in different neural crest streams, they arguably fail Paterson's (1982) test of ontogenetic similarity and could be considered nonhomologous. Theoretically, putatively homologous structures (e.g., lateral commissures, or trabeculae and visceral arches) could fail this test and still pass Patterson's (1982) other tests of homology (congruence, conjunction), if their developmental site shifted during evolution (heterotopy; Haeckel, 1875; Hall, 1998; Kuritani et al., 2004). To some extent, heterotopy is tautological since it makes a priori homology assumptions (presumably based on congruence and conjunction) about structures that display ontogenetic differences. On the other hand, ontogeny is the developmental phylogeny of an organism, and related taxa have congruent early ontogenies up to their points of divergence (von Baer's law, from the more similar to the less similar), so heterotopy may represent one of the key processes involved in stepwise phylogenetic transformations of ontogeny.

Postorbital Articulation: The postorbital articulation in Cladodoides is located entirely on cartilage presumably derived from the embryonic lateral commissure. The lateral head vein presumably passed medial or dorsomedial to this articulation. Such an arrangement has no exact modern counterpart, since a postorbital articulation is absent in neoselachians with a chondrified lateral commissure, but is present in hexanchiforms where the commissure is said to be absent (Holmgren, 1941). Thus, the articular surface in hexanchiforms apparently lies on the primary postorbital process rather than on the lateral commissure (Maisey, 2004b), and it is therefore farther dorsal (relative to the lateral head vein) than in Cladodoides, Tamiobatis, or Orthacanthus (in this regard, it seems to resemble the "auxiliary otic condyle" in Acanthodes). Furthermore, the articular surface in hexanchiforms is oriented posteroventrally rather than posteriorly. These differences suggest that the hexanchiform postorbital articulation has perhaps evolved independently from the primitive elasmobranch articulation. In this case, the presence of a postorbital articulation in hexanchiforms is not an "archaic" feature but may instead be a secondary specialization of the group (and it may be absent in early hexanchiforms such as Notidanoides; Maisey, 2004b). A postorbital articulation is absent in Chlamydoselachus (the putative sister taxon of hexanchiforms in some cladistic analyses), further suggesting that the articulation may be a synapomorphy of crown-group hexanchiforms. El-Toubi (1949) found evidence of an extraotic cartilage in Squalus, plastered against the lateral capsular wall to enclose part of the otic lateral line nerve and also fused to the postorbital process dorsally. He suggested that the extraotic cartilage is homologous to the palatoquadrate otic process in hexanchiforms, and he claimed that it is lateral to both the hyomandibular trunk and the jugular vein (although this is not evident from his illustrations). The cartilage in question appears first as a mesenchymatous band continuous with tissue surrounding the palatoquadrate farther ventrally. It then grows and chondrifies dorsally while atrophying ventrally, fusing secondarily with the otic capsule and trapping the otic lateral line ramus between them. It is unclear whether the extraotic cartilage is fused with the primary postorbital process or with the lateral commissure. Homology between the extraotic cartilage and part of the palatoquadrate is also doubtful, as Holmgren (1941: fig. 2) found a cartilageenclosed canal for the otic lateral line nerve in Heptranchias, where the postorbital articulation is present.

A postorbital articulation is typically absent in osteichthyans. One is present in Acanthodes, but it apparently differs from that of 
sharks in being located on the lateral wall of the sphenotic (Miles, 1965, 1973; Gardiner, 1984b). By contrast, in sharks the postorbital articulation is located on cartilage presumably either formed in the lateral commissure (e.g., Cladodoides) or on the primary postorbital process (e.g., modern hexanchiforms, which lack the lateral commissure in the adult; Holmgren, 1941; Maisey, 2004b). Although Miles (1973: figs. 4, 5) identified the lateral commissure in Acanthodes as lying medial rather than lateral to the postorbital articulation, such an arrangement is anatomically improbable if the articular surface is in fact located on the sphenotic (see above). In Acanthodes, there are two paired postorbital articular surfaces. One pair is located on the ventral part of the ossified postorbital process, and the second pair is slightly posterior on the lateral wall of the otic region (Miles, 1968, 1973; Jarvik, 1977). The articulation therefore extends over a much wider region in Acanthodes than in sharks, possibly encompassing part of the lateral commissure as well as the sphenotic.

The mandibular ramus of the trigeminal nerve in Acanthodes passed behind the articulation and penetrated the otic process of the palatoquadrate in order to reach the mandibular joint (Miles, 1965, 1971; Jarvik, 1977, 1980). However, in sharks this ramus either passes anterior to the postorbital process (hexanchiforms) or through it (in Cladodoides; see above). There is no evidence that the mandibular ramus penetrates the palatoquadrate in any modern or extinct elasmobranchs. The postorbital articulation is therefore anterior to the mandibular ramus in Acanthodes but posterior to it in elasmobranchs, and it is concluded that the postorbital articulation in Acanthodes differs from that of Paleozoic sharks and modern hexanchiforms in its extent, complexity, and relationship to the trigeminal mandibular ramus (irrespective of whether this articulation is homologous in all elasmobranchs).

The "Extra Foramen" Problem: Interpretation of arterial vessels in Paleozoic sharks is always problematic. Perennial difficulties (some of which are now potentially overcome by scanning technology) include: (1) incomplete or inaccurate data, stemming from inadequate preservation and prepara- tion of suitable fossils (e.g., the recent discovery of paired foramina for the lateral dorsal aortae in Cladoselache; Williams, 1998); (2) inability to see whether particular foramina are connected in fossils; and (3) disagreement over the identity of canals and foramina (e.g., for the palatine nerve, efferent hyoidean artery).

Williams (1998) presented an analysis of the basicranial arteries in several Paleozoic sharks in which foramina were simply identified numerically rather than by implied homologies (although any interpretation implies homology at some level). He drew attention to controversies surrounding previous interpretations and outlined what he termed the "extra foramen" problem in these early sharks (although much of this "problem" seems to be a product of differing opinions rather than extra openings). The problem was first articulated by Schaeffer (1981: 36), who suggested that the internal carotid and orbital arteries in Tamiobatis vetustus were originally enclosed completely by cartilage and that the efferent hyoidean emerged from the posteriormost of the paired basicranial foramina between the postorbital processes. Nevertheless, he admitted that an important difficulty for his interpretation is that "there appears to be an extra foramen ... between the palatine foramen and the one for the orbital artery on the rim of the suborbital shelf". Williams (1998) noted that two of the most controversial issues were (1) the separation of the orbital and internal carotid arteries, and (2) the separation of the efferent hyoidean artery. To these can now be added a third issue, that is, the position(s) of the facial nerve palatine ramus and its anterior and posterior ramules. Gross (1937) and Schaeffer (1981) reached very different conclusions regarding the identity of foramina for the orbital artery and the palatine branches of the facial nerve in Cladodoides, while Williams (1998) has presented a novel interpretation of the basicranial circuit in Tamiobatis vetustus which differs radically from that of Schaeffer (1981). Much of the disagreement in naming basicranial foramina in Paleozoic sharks seems to involve misinterpretation of the passages for the facial nerve's palatine ramules. Earlier interpretations are beset with assumptions about the 

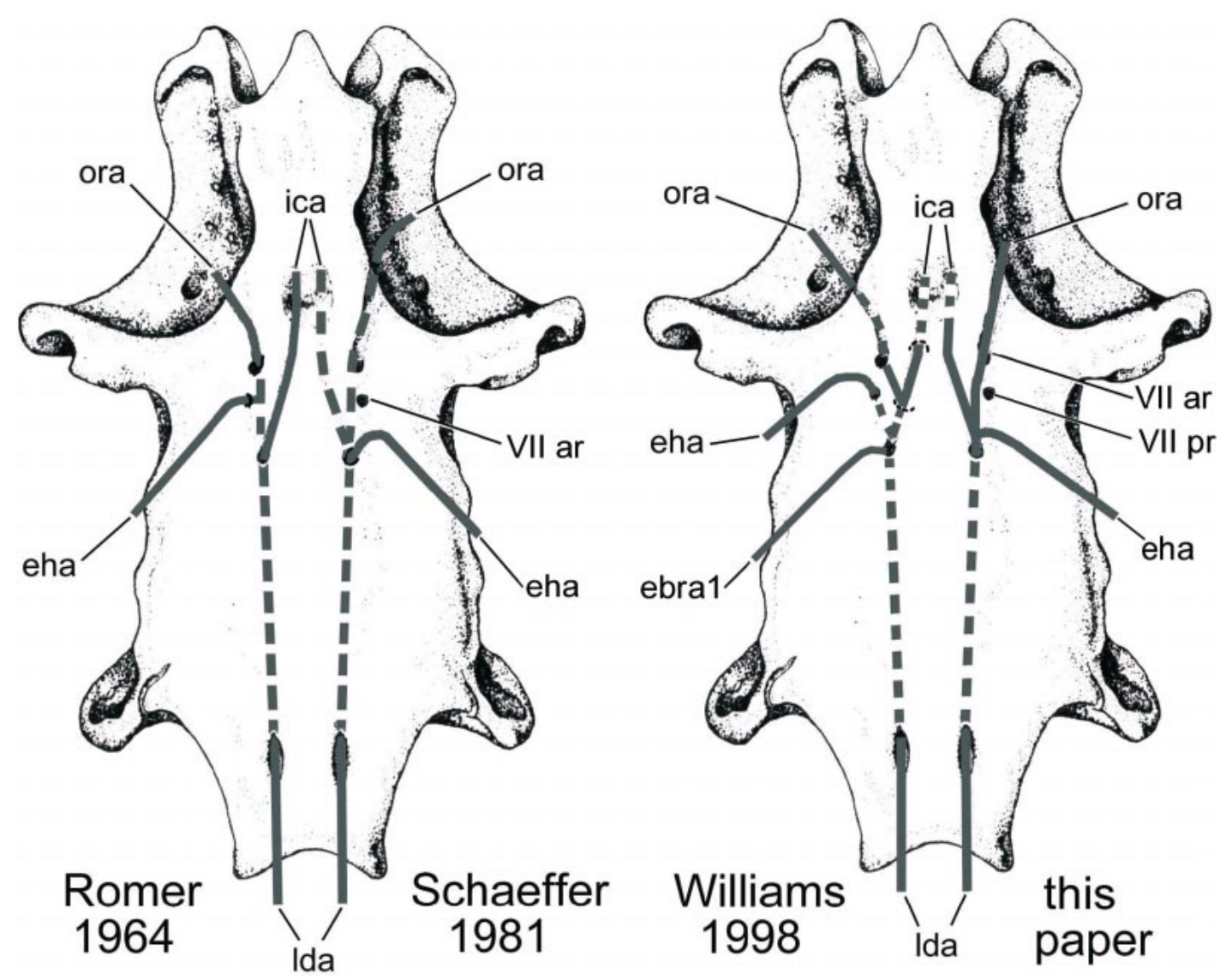

Fig. 35. Tamiobatis vetustus NMNH 1717. Ventral view of braincase, depicting four alternative scenarios of basicranial arteries discussed in the text. Note disagreements over the foramen for the orbital artery/anterior palatine ramus, site of efferent pseudobranchial foramen, and the extent to which internal carotid and orbital arteries were enclosed by cartilage. The preferred arrangement is similar to that reconstructed in Cladodoides (fig. 21). No scale.

extent of missing cartilage and the supposed directions of hidden passages.

Three different interpretations of the basicranial circuit in Tamiobatis vetustus have been presented by Romer (1964), Schaeffer (1981), and Williams (1998). These are depicted diagrammatically, together with a novel fourth interpretation, in figure 35. Romer (1964) did not illustrate the circuit, and the interpretation shown here is therefore based only on his description. These hypotheses differ principally in the interpretation of what Williams (1998) termed "foramen two" and "foramen three". Unfortunately, the ventral surface of NMNH 1717 is damaged and many of its features are lost apart from deep- er channels around passages for nerves and blood vessels. Romer (1964) recognized that the paired lateral aortae ("common carotids") were largely enclosed by cartilage. He also suggested that the internal carotids emerged from the braincase before passing toward the bucco-hypophyseal fenestra. Farther laterally, he identified an opening for "the hyomandibular (pseudobranchial) artery" (i.e., the efferent hyoidean) and considered that the orbital artery lay in a deep groove, leading forward and outward to the orbit.

Williams (1998) addressed the "extra foramen" problem in Tamiobatis vetustus and offered an alternative interpretation in which 
the efferent hyoidean and first efferent branchial arteries branched from the lateral aorta within the floor of the braincase and emerged via separate foramina. The lateral aorta then supposedly emerged from the cartilage before branching into the internal carotid and orbital arteries, which then reentered the cartilage farther anteriorly. Two criticisms may be leveled at his reconstruction: first, it is based on a different specimen, which while well preserved may not represent Tamiobatis vetustus; and second, his reconstruction also requires several assumptions about hidden passages and the "extra" foramen. His interpretation of CMNH 9280 has been superimposed on NMNH 1717, but this is not altogether satisfactory because the orbital artery would have to enter the braincase at precisely the point it seems to leave in Romer's (1964) version, and the supposed efferent branchial and efferent hyoidean foramina in CMNH 9280 could not have housed these vessels in NMNH 1717. The arrangement of basicranial openings in CMNH 9280 differs slightly from NMNH 1717, and the arterial circuit may not be identical, but examination of CMNH 9280 nevertheless suggests that the supposed efferent hyoidean foramen actually housed the posterior ramule of the palatine ramus and that the anterior ramule joined the orbital artery in a notch at the point where the suborbital shelf meets the postorbital process.

The branching pattern of the palatine ramus passage in Cladodoides is crucial to understanding the extra foramen problem in Tamiobatis vetustus and other Paleozoic sharks. The position of the orbital artery foramen in Cladodoides is very similar to that proposed by Williams (1998: "foramen one") in T. vetustus. In Cladodoides, the anterior palatine ramule and orbital artery converged just as the former emerged from the braincase in front of the postorbital process, so their passages share a common exit although they remained separate (the canal shows that one lay above the other). In Cladodoides, the anterior and posterior ramules of the palatine ramus probably occupied foramen one and foramen two, respectively, of Williams (1998). His interpretation that foramen two housed the efferent hyoidean (as suggested in Tamiobatis vetustus by Romer,
1964) is not feasible in Cladodoides, and in my view the corresponding foramen in Tamiobatis vetustus (and many other Paleozoic sharks, including "Cladodus" hassiacus and Cladoselache) probably also contained the posterior ramule of the palatine ramus. Stensiö (1937) identified "posterior" and "palatine" passages in the Humboldt University braincase, suggesting that here too the anterior and posterior ramules of the palatine ramus both passed through the basicranium.

An alternative basicranial reconstruction in Tamiobatis vetustus is therefore presented here, with (as in Cladodoides) separate anterior and posterior ramules rather than a single palatine ramus (explaining the "extra" foramen in $T$. vetustus). In addition, contrary to popular opinion, no trace of a canal or groove for the orbital artery is preserved in NMNH 1717. The supposed groove for the orbital artery is very deep and probably represents the roof of a canal for the anterior palatine ramule, whose floor has probably been stripped away by erosion. Crucially, this passage is directed posterodorsally toward the foramen for the posterior palatine ramule. The orbital artery in $T$. vetustus probably lay below the anterior palatine ramule, and both entered the orbit one above the other, as in Cladodoides. It is also possible that the orbital artery was enclosed by a canal for at least part of its length (possibly a growth-related feature as in the Humboldt University braincase).

Some differences are noted in the arrangement of basicranial foramina in NMNH 1717 and the specimen referred to Tamiobatis vetustus by Williams (1998: CMNH 9280), suggesting there was some variation in the courses of the orbital and efferent hyoidean artery and of the palatine ramus of the facial nerve, although it is uncertain whether this is because different taxa are represented. Some aspects of the basicranial pattern postulated by Williams (1998) in CMNH 9280 may have been incorrectly identified, or other factors (e.g., ontogenetic or preservational differences) may be involved.

Akmonistion has been interpreted with a separate efferent hyoidean foramen, suggesting that the artery arose from the lateral aorta within the basicranium (Coates and Sequeira, 1998). However, there is no evidence of such 
an arrangement in the "Cobelodus" braincase (Maisey, 2004a and in prep.). In chimaeroids, the branchial arteries all meet the aortic circuit below the braincase (De Beer and Moy-Thomas, 1935), but in Pucapampella an efferent hyoidean or branchial artery may have branched from the lateral aorta within the parachordal plate (Janvier and Suárez-Riglos, 1986; Maisey, 2001c). Reconstructions of sharks such as Tamiobatis vetustus showing an efferent hyoidean foramen are probably erroneous (e.g., Romer, 1964; Schaeffer, 1981; Williams, 1998).

REMARKS ON THE DORSAL VEIN SINUS IN ACANTHODES: In Acanthodes, the anterior ventral ossification (generally considered homologous to the basisphenoid in osteichthyans; Miles, 1973; Gardiner, 1984a; Janvier, 1996) contains a median basal fenestra in the center of a shallow hypophyseal fossa. Miles (1973) and Jarvik (1977) agreed that the internal carotids converged on the basal fenestra ventrally, then entered the ossification and emerged on its dorsal surface. However, they differed in their interpretations of the position of the efferent pseudobranchial. According to Jarvik (1977), the internal carotids met the efferent pseudobranchial (and ophthalmic arteries) above the trabecular-polar plate in elasmobranch fashion, but Miles (1973) identified a groove for the efferent pseudobranchial on the ventral surface of the basisphenoid, suggesting that it met the internal carotid ventral to the trabeculae, as in osteichthyans.

Jarvik's (1977) interpretation of features on the ventral surface of the basisphenoid was strongly influenced by his proposal that a neoselachian-like dorsal median vein sinus was present in Acanthodes. However, his interpretation is equivocal and assumes that paired basicranial grooves originally housed buccopharyngeal veins. There is no direct evidence for the presence of a dorsal median vein sinus in Cladodoides or any other extinct shark and it is therefore impossible to determine the distribution of the sinus outside modern neoselachians. Even if a dorsal vein sinus were present in Acanthodes, its value as a potential synapomorphy of Acanthodes and elasmobranchs would remain dubious (irrespective of its significance as an apomorphic or plesiomorphic character within elasmobranchs).

Otico-Occipital Proportions: Romer (1964) and Coates and Sequeira (1998) suggested that a comparatively long otico-occipital region is probably a primitive gnathostome condition, but they disagreed as to whether the elongate otic region in Tamiobatis vetustus is primitive (Romer, 1964) or a reversal (Coates and Sequeira, 1998). A relatively short otico-occipital region commonly occurs in actinopterygians, Pucapampella, chimaeroids, neoselachians, and hybodonts, lending circumstantial support to the second alternative. The apparent length of the otico-occipital region is affected by the position of the postorbital process, although other factors are also involved, including (1) the anteroposterior extent of the semicircular canals, (2) the angle at which the anterior and posterior canals diverge, and (3) the relative extent to which the occipital arch intrudes between the capsules. Schaeffer (1981: fig. 16) attempted to illustrate this by superimposing the endocasts of Squalus and Orthacanthus, but it is not possible to characterize these differences in a straightforward manner. The otico-occipital proportions in Orthacanthus, Tamiobatis vetustus, and "Tamiobatis sp." are nevertheless very similar and Schaeffer (1981) suggested that this may represent a synapomorphy of these taxa. In all these forms, the otico-occipital region is longer (with respect to orbit length) than in Cladodoides. The hypotic lamina was also long in Cladodus elegans, and its otic region may therefore have been proportioned as in Tamiobatis vetustus and "Tamiobatis sp." The posterior extremity of the Humboldt University braincase is missing and its proportions cannot be determined.

REMARKS ON THE INNER EAR IN CHONDRICHTHYANS, PlaCODERMS, AND AgNATHANS: In craniates generally, the semicircular canals initially develop within the thickness of the capsular walls, whereas the major vestibular chambers (utriculus, sacculus, lagena) lie inside the capsule. This represents a fundamental morphological and developmental characteristic of the labyrinthine and vestibular parts of the inner ear. The ampullae (and the utricular recess, where present) represent major points of entry for the canals into the 
main vestibular chambers. Sensory cristae are primitively located at or near these points of entry and the octaval nerve typically does not extend beyond the ampullae inside the capsular wall. The external (horizontal) semicircular canal is found only in gnathostomes (including placoderms) and its absence in cyclostomes (and all ostracoderms where the inner ear is known) presumably represents the primitive craniate condition.

In neoselachians, the medial capsular wall is fully chondrified (and usually well calcified) apart from the octaval and glossopharyngeal foramina. However, its development is completed fairly late in ontogeny (De Beer, 1931; Holmgren, 1940; Jollie, 1971). The medial capsular wall is also chondrified in Mesozoic hybodonts (e.g., Hybodus, Tribod$u s$ ) although its extent in earlier hybodonts such as Hamiltonichthys is unknown. Probably the medial wall was partly or even completely chondrified in Tristychius, since Dick (1978: figs. 2, 4) showed extensive chondrification on each side of the "median supraotic fossa" extending into the braincase medial to the capsules. Chondrification of the medial capsular wall effectively isolates the skeletal labyrinth from the main cranial cavity, so their endocasts are essentially separate. Digitally generated endocasts of the skeletal labyrinth in the modern broadnose sevengill shark Notorynchus and the Cretaceous hybodont Tribodus are illustrated in figure 36A-F.

By contrast, most of the medial capsular wall is unchondrified in Cladodoides, apart from the region immediately adjacent to the octaval nerve (fig. 36G). It could be argued that lack of chondrification in this particular braincase is a juvenile characteristic, especially since chondrification is also incomplete elsewhere. However, the medial capsular wall is also unchondrified or weakly chondrified in other Paleozoic sharks (e.g., the Pennsylvanian braincase referred to "Cobelodus"; fig. 36H, and in Cobelodus aculeatus, "Tamiobatis sp.", Orthacanthus; Zangerl and Case, 1976; Schaeffer, 1981), as well as in Pucapampella (Maisey, 2001c; Maisey and Anderson, 2001), in modern holocephalans, and in osteichthyans (fig. 37A). Absence of a chondrified medial capsular wall is therefore a widespread feature among crown-group gnathostomes and could therefore be regarded as a plesiomorphic feature at this level. Nevertheless, the medial capsular wall is fully ossified in many placoderms (fig. 36I), osteostracans, and galeaspids, suggesting somewhat paradoxically that the wall is primitively present in stem gnathostomes. Presence of a chondrified or ossified medial capsular wall therefore has a disjunct distribution among modern and extinct craniates and it may have arisen more than once in craniate history, but its presence in phylogenetically advanced elasmobranchs (neoselachians, hybodonts) is unique among crown-group gnathostomes.

In neoselachians, the medial capsular wall forms mainly in the taenia medialis, which arises from the synotic tectum and extends down between the brain and labyrinth to fuse with upgrowths from the parachordal plate (e.g., Scyliorhinus; De Beer, 1931). Thus, absence of a medial capsular wall in extinct sharks may perhaps be more accurately defined as absence of chondrification in the taenia medialis. As discussed earlier, the internal wall of the acustico-trigemino-facial recess in Cladodoides is part of the prefacial commissure, not the taenia medialis. This may also be the case in Cobelodus aculeatus, where the ganglia of the octaval, trigeminal, and facial nerves all lie within a shallow recess located in a vertical septum separating the anterior part of the vestibular chamber from the main cranial cavity (Zangerl and Case, 1976: fig. 9B). Transverse sections of the braincase in Orthacanthus (Schaeffer, 1981: fig. 9) suggest very limited chondrification in the floor of the sacculus and the lateral walls of the posterior dorsal fontanelle. There is no evidence of a continuous cartilaginous taenia medialis extending from the fossa to the basicranium, but traces of calcified cartilage between the brain and labyrinth cavities suggest that the membranous taenia in Orthacanthus was as extensive as in neoselachians. In the "Tamiobatis sp." braincase (AMNH 2140, unfortunately studied only in horizontal sections which are difficult to compare with the transverse slices of Orthacanthus), the synotic tectum is chondrified and forms the anterior margin of the posterior dorsal fontanelle, but there is little 


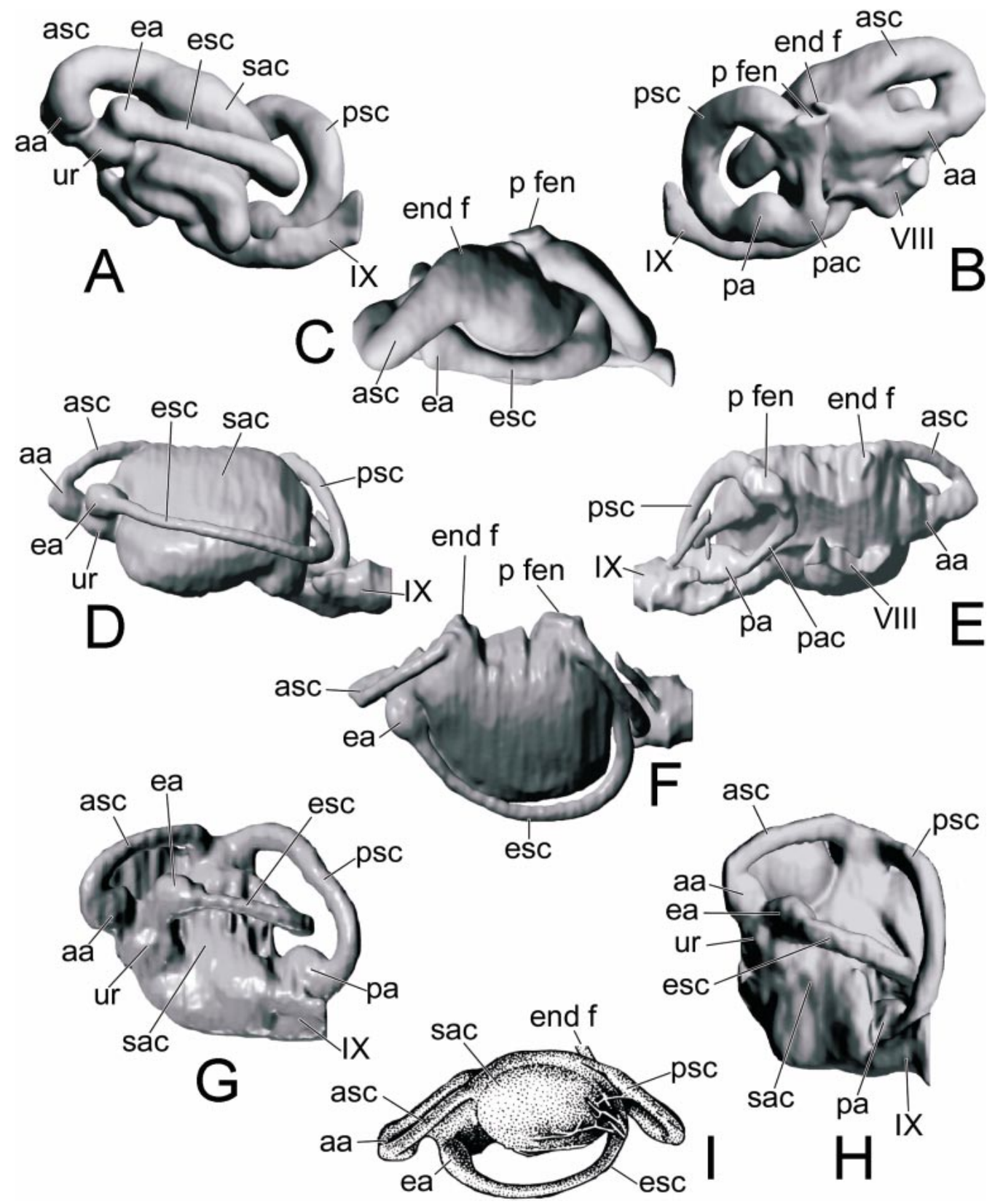

Fig. 36. Endocasts of the left skeletal labyrinth in: (A-C) modern Notorynchus, lateral (A), medial (B), and dorsal (C) views (after Maisey, 2004); (D-F) a Cretaceous hybodont, Tribodus, lateral (D), medial(E), and dorsal (F) views (original); (G) Cladodoides, lateral view; (H) a Pennsylvanian symmoriid, "Cobelodus", lateral view; (I) the arthrodire Kujdanowiaspis, dorsal view (after Stensiö, 1963a). Not to scale. 


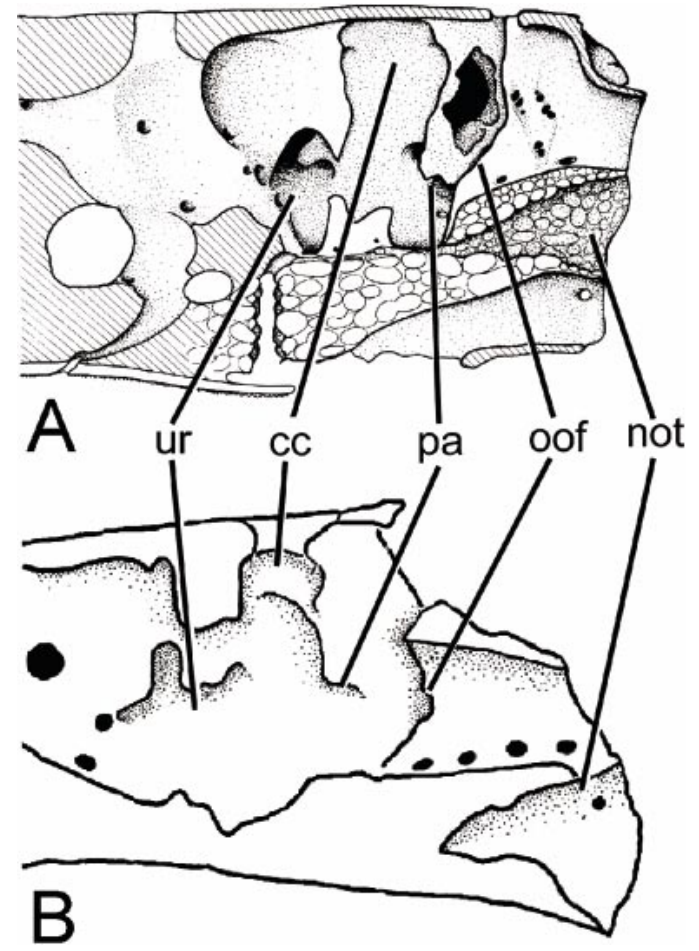

Fig. 37. Otico-occipital regions in medial view as if sliced sagitally, in (A) an early actinopterygian, Mimia (after Gardiner, 1984a); (B) Cladodoides. Note general similarity in vestibular chamber morphology, including presence of a septum incompletely separating the utricular recess and medullary chamber dorsally. Not to scale.

evidence of a cartilaginous taenia medialis deeper within the braincase.

There is a rather wide communication between the cranial and labyrinth cavities in Cladodoides (figs. 7, 21A, D), the primitive actinopterygian Mimia (Gardiner, 1984a: fig. 26), and Eusthenopteron (Jarvik, 1980), although in all these forms parts of the labyrinth are enclosed by the walls of the otic region. There is also an extensive saccular recess, but in Mimia this is partly separated from the cranial cavity ventrally and slightly posterior to the level of the utricular recess (Gardiner, 1984a: 227). In both Cladodoides and Mimia, the utricular recess and metencephalic chamber are partly separated by a septum, at the base of which the hyomandibular ramus of the facial nerve probably left the cranial cavity (figs. 7, 35, 37; see also
Gardiner, 1984a: fig. 26, fhm). The trigeminal nerve and anterodorsal lateral line trunk left the cranial cavity farther dorsally; in Mimia these had separate foramina, but in Cladodoides they apparently shared a single opening (V, adll, fig. 7; Gardiner, 1984a: fig. 26, VII lat, V). In both Mimia and Cladodoides, the septum is notched for the utricular recess posteroventrally (fig. 37). In Mim$i a$, the septum and zygal plates help define the dorsal and ventral margins of a wide fenestra leading to the utricular recess, through which the octaval nerve must have passed. Although the ventral part of this fenestra is poorly defined in Cladodoides, it is nevertheless possible to envisage a comparable arrangement to Mimia since its octaval nerve apparently entered the recess in a corresponding position (this can be seen in CT scan section 161; fig. 18E). In Cladodoides, therefore, this entire region corresponds to the chondrified acustico-trigemino-facial recess described in modern sharks by Allis (1914, 1923a). However, in neoselachians this septum forms a more extensive medial capsular wall, almost completely separating the utricular recess from the metencephalic chamber. Such an arrangement probably represents a derived condition within crowngroup gnathostomes, whereas the less extensive septum in Cladodoides and Mimia probably represents a conserved gnathostome character.

The view of the endocranial wall in $C o b-$ elodus aculeatus depicted by Zangerl and Case (1976: fig. 9B) also shows a septum (presumably between the utricular and metencephalic chambers and supposedly containing the trigeminal, abducent, facial, and octaval nerves). However, in the "Cobelo$d u s "$ braincase, the septum is chondrified only dorsally and does not extend to the facial foramen (in prep.). Without going into details, the arrangement depicted by Zangerl and Case is probably incorrect and an alternative interpretation will be presented elsewhere.

The neoselachian posterior semicircular canal displays several unique or unusual features among extant craniates (Maisey, 2001b; see also fig. 36A-C here), including:

1. The ampullary and nonampullary ends of the 
posterior semicircular canal are connected by an almost vertical preampullary canal (= "posterior oblique semicircular canal" of Daniel, 1934), which extends anteriorly and upward from the posterior ampulla. Consequently, the posterior ampulla no longer represents the point at which the semicircular canal enters the vestibular chamber. A short preampullary canal is also present in some other crowngroup gnathostomes including chimaeroids and Acipenser (Retzius, 1881), but it never extends very far dorsally and does not form a complete loop as in neoselachians. A preampullary canal is well developed in some placoderms (e.g., Kujdanowiaspis, Tapinosteus; Stensiö, 1963a) but not in others (e.g., Brindabellaspis, Dicksonosteus; Young, 1980; Goujet, 1984).

2. In neoselachians, the posterior semicircular canal is contained almost completely in the chondrified medial capsular wall instead of lying partly within the vestibular chamber (as in chimaeroids and osteichthyans). A remarkably similar arrangement occurs in placoderms and ostracoderms (e.g., Mimetaspis [Cephalaspis], Kiaeraspis; Stensiö, 1927), where the medial capsular wall is also fully ossified apart from the glossopharyngeal and octavolateral canals.

3. The neoselachian posterior semicircular canal has only a single connection with the vestibular chamber via the posterior canal duct associated with the macula (crista) neglecta (Corwin, 1989). This duct is sometimes quite long and separates the canal from the remainder of the labyrinth (e.g., batoids; Bleckman and Hoffman, 1999). In osteichthyans, chimaeroids, osteostracans, and galeaspids the posterior semicircular canal enters the sacculus ventrally and meets the anterior semicircular canal dorsally, but in some arthrodires the posterior canal may describe a full circle as in neoselachians.

4. A short section of the neoselachian posterior semicircular canal is exposed in the lateral wall of the parietal fossa and may even bulge into the perilymphatic fenestra. Perilymphatic fenestrae are absent in modern osteichthyans, chimaeroids, and cyclostomes and have not been identified in placoderms or ostracoderms. According to Dick (1978), a posterior opening in the dorsal fontanelle of Tristychius may have contained perilymphatic fenestrae, but the opening in question is median rather than paired and may simply represent an unmineralized area of the fontanelle floor.

5. The neoselachian anterior and posterior semicircular canals do not meet at a crus commune dorsally. By contrast, a crus commune is present in chimaeroids and osteichthyans, extinct chondrichthyans (e.g., Cladodoides, Pucapampella; Maisey, 2001c), lampreys, and many osteostracans (Janvier, 1981a, 1981b, 1985). The hagfish labyrinth includes a single canal containing both anterior and posterior sensory cristae, suggesting that it represents the combined anterior and posterior canals (Maisey, 2001b). A crus commune is supposedly absent in placoderms (Stensiö, 1950, 1963a, 1963b, 1969).

6. The neoselachian anterior and external ampullae are said to share a single utriculo-saccular connection with the utricular recess, instead of having separate openings as in other gnathostomes (Torrey, 1962). However, it is uncertain whether this feature characterizes all or only some neoselachians.

Stensiö (1950, 1963a) found several similarities in the inner ear of arthrodires and neoselachians, including their widely separated anterior and posterior semicircular canals, enclosure of the posterior canal by the medial capsular wall, and presence of an ascending preampullary canal (e.g., Kujdanowiaspis, Tapinosteus). However, several important differences can also be identified in the semicircular canal arrangement in arthrodires and neoselachians, including:

1. In some arthrodires there is a crus between the external and anterior semicircular canals (as in neoselachians), but the external canal is also connected medially to the posterior one by a narrow anteriorly directed commissure (e.g., Kujdanowiaspis; fig. 36I). Young (1986: 41) concluded that the dorsomedial "ridge-like expansion of the sacculus in Kujdanowiaspis and Tapinosteus is more suggestive of a semicircular canal extension, [and] it could ... have contained an anterior extension of the posterior canal". If that is correct, the posterior canal was not isolated as in neoselachians and instead continued forward to meet the anterior one. The arthrodire dorsomedial canal is not homologous to the neoselachian posterior canal duct, since it does not open into the upper part of the vestibular chamber ("posterior utriculus"). Thus, even if the posterior semicircular canal describes a complete circle in both neoselachians and arthrodires, its connection to the rest of the labyrinth is different.

2. The superficial position of the external semicircular canal around the dorsomedial part of the capsule in arthrodires (e.g., Kujdanowiaspis, Tapinosteus; Stensiö, 1963a) contrasts with its much deeper position in crown-group gnathostomes, where the external semicircular 
canal turns inside the loop of the posterior canal, and its nonampullary end cannot be traced deeper in the endocast. In modern osteichthyans and chimaeroids (Retzius, 1881), as well as in Cladodoides, "Cobelodus", and Pucapampella, the external semicircular canal meets the utriculus below the crus commune (e.g., fig. 36G, H). In arthrodires, therefore, the medial end of the external semicircular canal is positioned more superficially (relative to the rest of the labyrinth) than in crown-group gnathostomes, and it can be traced for its entire length fig. 36I). In many osteostracans and galeaspids, the anterior and posterior semicircular canals are again superficial and can be traced from their ampullae all the way to the crus commune and saccular chamber (e.g., Cephalaspis hoeli, Norselaspis, Duyunolepis, Xiushuiaspis, Benneviaspis, "Boreaspis"; Stensiö, 1927; P' an and Wang, 1978; Janvier, 1981a, 1981b, 1984, 1985; Wang, 1991).

3. No perilymphatic fenestrae have been found in placoderms. These fenestrae form an essential part of the phonoreceptor system in neoselachians and so far have not been identified in other craniates (Maisey, 2001a).

4. In some arthrodires, the anterior semicircular canal meets the utricular recess indirectly via a short anterior preampullary canal (e.g., $\mathrm{Ku}$ jdanowiaspis, Tapinosteus). In other arthrodires the anterior preampullary canal is absent (e.g., Brindabellaspis, Dicksonosteus). No such canal has been found in extant or fossil chondrichthyans. Presence of this canal in arthrodires may have phylogenetic importance.

The following scenario is suggested by these observations: the anterior and posterior semicircular canals were primitively situated entirely within the walls of the otic capsule in early craniates (a pattern that is conserved in lampreys, osteostracans, and galeaspids); the external semicircular canal of gnathostomes subsequently arose in a similarly superficial position (a pattern that is conserved in arthrodires); the medial part of the external semicircular canal became located deeper in the labyrinth in the common ancestor of crown-group gnathostomes (a pattern that is conserved in osteichthyans, chimaeroids, and early sharks); and decoupling of the posterior semicircular canal occurred late in elasmobranch history (a pattern that is conserved in neoselachians and hybodonts).

In Cladodoides, osteichthyans, and chimaeroids, an ascending posterior preampul- lary canal is absent, the medial capsular wall is membranous, the anterior and external ampullae meet the utricular recess separately, the posterior canal meets the sacculus ventrally and meets the anterior canal at a crus commune, and perilymphatic fenestrae are absent. Importantly, no apomorphic features of the inner ear have been identified that are shared only by placoderms and cladistically primitive chondrichthyans, while the few unusual placoderm features (e.g., the superficial position of the dorsomedial canal, anterior preampullary canal) are absent in early elasmobranchs. It is concluded that those similarities noted in the past between the labyrinth of arthrodires and neoselachians include only conserved craniate and gnathostome characters, plus some convergent features that are absent in more primitive chondrichthyans. Arthrodire inner ear morphology provides no convincing morphological evidence of a phylogenetic relationship with elasmobranchs or chondrichthyans, and while it may eventually provide informative characters for resolving arthrodire or placoderm phylogeny, it does not help resolve their wider relationships other than to suggest that arthrodires are plesiomorphic stem gnathostomes.

Posterior Dorsal Fontanelle and PosTERIOR TECTUM: The dorsal fontanelle in extinct sharks is commonly identified as a parietal or endolymphatic fossa, like that of modern elasmobranchs. However, the neoselachian parietal fossa forms part of a highly specialized, low-frequency, semidirectional phonoreceptor system that also involves many other structures in the otic region (Corwin, 1989; Maisey, 2001b). The fossa is filled with a sound-transmitting gel and communicates directly with the posterior semicircular canal (which is almost completely isolated from the remainder of the labyrinth; see below) via paired perilymphatic fenestrae. These are actually unchondrified regions in the medial capsular wall through which the posterior semicircular canal protrudes, forming a tympanic membrane that transmits low-frequency soundwaves to the crista neglecta (De Beer, 1931, 1937; Holmgren, 1940; Corwin, 1989). Short perilymphatic ducts may extend from the fenestrae and are sometimes evident in the endocast of 
the skeletal labyrinth (fig. 36B, C, E, F). There is no evidence of perilymphatic fenestrae or ducts in Cladodoides. A low-frequency phonoreceptor system was probably absent in many Paleozoic sharks, and these forms probably relied on lateral line receptors to detect pressure waves (as in most osteichthyans).

Maisey and Anderson (2001) noted that the fontanelle in Pucapampella closely resembles the osteichthyan posterior dorsal fontanelle (see Gardiner, 1984a) and suggested that presence of the posterior dorsal fontanelle may be a synapomorphy of crowngroup gnathostomes. No comparable fontanelle has been identified in placoderms. In Acanthodes, there is a median "anterior dorsal fontanelle" between the postorbital processes (i.e., farther anterior than the posterior dorsal fontanelle), that is supposedly related to the pineal organ (Miles, 1973: fig. 3). A posterior dorsal fontanelle may be represented by a slight widening of the dorsal part of the otico-occipital fissure (Miles, 1973: fig. 3; Jarvik, 1980: fig. 258). However, Jarvik's (1977, 1980) reconstruction showing a neoselachian-like endolymphatic fossa separated from the otico-occipital fissure by a wide tectum in Acanthodes is entirely speculative and seems to have resulted from over-reliance of morphological data obtained from Recent rather than from early elasmobranchs. The parietal fossa is a specialized structure and is known with certainty only in neoselachians and hybodonts, suggesting that the posterior dorsal fontanelle probably became secondarily conscripted as part of the phonoreceptor system comparatively late in elasmobranch evolution (Maisey and Anderson, 2001). Endolymphatic ducts are present in chimaeroids (as in gnathostomes generally), but chimaeroid inner ear morphology lacks numerous specialized features found in neoselachians. Consequently, there is no reason to suppose that chimaeroids possessed a parietal fossa at any stage in their history, although they may have primitively possessed a posterior dorsal fontanelle.

From these observations, it is suggested that: a posterior dorsal fontanelle and oticooccipital fissure were primitively absent in craniates, and that this pattern was conserved by some early gnathostomes including pla- coderms; a posterior dorsal fontanelle was connected to a persistent otico-occipital fissure without an intervening posterior tectum in more crownward gnathostomes (a pattern that was conserved in Acanthodes, osteichthyans, and early chondrichthyans such as $\mathrm{Pu}$ capampella and Orthacanthus); the posterior dorsal fontanelle and the otico-occipital fissure became ontogenetically closed independently in several osteichthyan lineages and in chimaeroids; the posterior dorsal fontanelle became separated from the otico-occipital fissure by a posterior tectum in elasmobranchs (a pattern that is conserved in Cladodoides and Tamiobatis vetustus); and the posterior dorsal fontanelle became conscripted into a low-frequency phonoreceptor system, becoming the parietal fossa, and the otico-occipital fissure became closed in ontogeny (e.g., neoselachians, hybodonts).

The ontogenetic origin of the posterior tectum is unclear. Holmgren (1940: fig. 122) illustrated the 62-mm stage of Heterodontus in dorsal aspect, in which the otico-occipital fissure separates the occipital pila from the otic capsules and there are large paired fenestrae (presumably representing the combined endolymphatic and perilymphatic openings within the parietal fossa). The posterior part of the fossa is still connected to the oticooccipital fissure even though a medial tectum connects the synotic and occipital tectum posteriorly. The medial tectum forms the floor of the parietal fossa and is eventually connected to the otic capsules. It is possible that the posterior tectum in Cladodoides represents a medial tectum which has expanded laterally to connect the otic capsules in front of the occipital fissure. Diagrammatic representations of these conditions are shown in figure 38.

LAteral Otic Process: The lateral otic process represents an extension of the posterolateral capsular wall and contains the posterior semicircular canal (Schaeffer, 1981). This process is well developed in many Paleozoic sharks, including Orthacanthus, Tamiobatis vetustus, "Tamiobatis sp.", and Akmonistion. It was probably present in Cladodoides although its extent is unknown (see above). Part of the lateral otic process is preserved next to the occipital arch in one of the specimens referred to Cladodus elegans. 


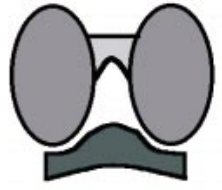

A

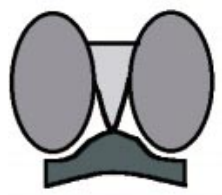

C

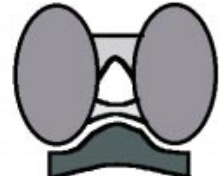

B

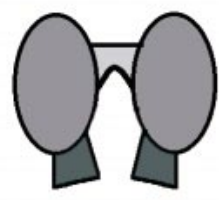

D
Fig. 38. Diagrammatic representations of the posterior dorsal fontanelle, otic capsules, synotic tectum, and occipital arch in various elasmobranchs. Dorsal views, anterior to top: (A) $P u$ capampella or Tamiobatis; posterior tectum absent, fontanelle confluent with otico-occipital fissure, occipital arch complete; (B) Cladodoides; posterior tectum present, fontanelle separated from fissure, occipital arch complete; (C) 62-mm Heterodontus; medial tectum extends from synotic tectum and meets complete occipital arch prior to its fusion with otic capsules (D) 48-mm Squalus; occipital pilae fused to otic capsules prior to closure of occipital arch dorsally. Panels C and D are based on figures in Holmgren (1940). Not to scale.

The process may also form part of the hyomandibular fossa.

It has been claimed that a lateral otic process is present in neoselachians (Schaeffer, 1981) and in Hybodus (Maisey, 1983), while Coates and Sequeira (1998: 79) regarded it as "almost ubiquitous" among chondrichthyans. However, according to the above definition there is no lateral otic process in neoselachians or hybodonts. Instead, in neoselachians there is a postotic process (sensu Holmgren, 1941) that lies dorsal to the hyomandibular fossa and contains the glossopharyngeal nerve but not the posterior semicircular canal. A postotic process only occurs in elasmobranchs with a glossopharyngeal canal and is absent in forms with a persistent metotic fissure (e.g., Orthacanthus, Tamiobatis). Its ontogeny in neoselachians involves fusion of the ventral part of the capsular wall and the hypotic lamina. By contrast, the lateral otic process is formed entirely on the lateral capsular wall, and both the process and the hyomandibular fossa are separated from the hypotic lamina by the ventral otic notch. Based on these topographic and ontogenetic differences, the postotic process in neoselachians is not considered homologous to the lateral otic process in Paleozoic sharks.

The supposed lateral otic process in $\mathrm{Hy}$ bodus resembles the postotic process in neoselachians in lying dorsal to the hyomandibular fossa, but it does not contain the glossopharyngeal nerve or the posterior semicircular canal (Maisey, 1983). On that basis, it probably does not represent either a lateral otic process or a postotic process; instead, it merely seems to be an outgrowth of the lateral capsular wall which supports a dermal cephalic spine in males.

In some neoselachians, the hyomandibular fossa may be sandwiched between the postotic process dorsally and a rather featureless vestibular process ventrally (Gadow, 1888). A vestibular process has not been identified in Cladodoides or any other Paleozoic shark. The vestibular process may contribute to the ventral margin of the hyomandibular fossa (e.g., the "Basalrande des Hyoidgelenkes" in Squatina; Iselstöger, 1937), but it does not contain the posterior semicircular canal or the glossopharyngeal nerve.

To summarize, several different kinds of process may be developed on the lateral or posterolateral wall of the otic capsule in elasmobranchs, but the lateral otic process uniquely encloses part of the posterior semicircular canal. This process has a more restricted distribution than previously supposed and may help define a monophyletic group within elasmobranchs.

HYOMANDIBULAR Fossa: In modern batoids and orbitostylic sharks, the hyomandibular fossa is located on the posterior part of the otic capsule, immediately in front of the glossopharyngeal foramen and below the ridge for the external semicircular canal. The exact position of the fossa is uncertain in Cladodoides (see above), but in Hybodus, Tribodus, Orthacanthus, Tamiobatis vetustus, "Tamiobatis sp.", and Akmonistion it is located on the posterior part of the capsular wall. However, in modern Heterodontus, galeomorphs, and in the extinct neoselachian Synechodus (also perhaps a galeomorph; 
Maisey, 1985; Maisey et al., 2004), the fossa is farther anterior, on the side wall of the otic capsule. In Tristychius, the hyomandibular fossa is directly behind the postorbital process (Dick, 1978), and Falcatus supposedly has the articular fossa on the postorbital process (Lund, 1985). Stensiö (1937) identified the hyomandibular fossa in the Humboldt University braincase in an anterior position on the otic capsule, immediately posterior to the facial foramen. However, it seems more likely that this area corresponds to part of the lateral otic fossa in the Senckenberg braincase and that the hyomandibular fossa probably lay much farther posteriorly in the Humboldt University specimen. The arrangement in Tristychius is remarkable since the suspensorial arrangement restored by Dick (1978: fig. 14) is clearly present in several articulated specimens and differs profoundly from modern elasmobranchs.

Zangerl (in Zangerl and Williams, 1975; Zangerl and Case, 1976) interpreted the symmoriid shark Cobelodus aculeatus as aphetohyoidean (sensu Watson, 1937) and proposed that the hyomandibula was nonsuspensory in primitive sharks. However, Williams (1985) noted that in Denaea meccaensis (which he considered to be another symmoriid), the proximal end of the hyomandibula almost certainly articulated with the posterolateral part of the otic capsule. Subsequently, Zangerl (1990: 119 et seq.) described the hyomandibula in Stethacanthulus (a small, somewhat unusual stethacanthid) as the "standard symmoriid type", with a "proximal joint surface ... attached to the lateral otic (epiotic) process on the neurocranium", and he characterized stethacanthids and symmoriids as having the hyomandibula "articulating at the otic process of the neurocranium."

Aside from the few controversial findings discussed above, the hyomandibular fossa in fossil and modern elasmobranchs is usually located on the posterolateral wall of the otic capsule. It is also in this position in the primitive chondrichthyan Pucapampella (Maisey and Anderson, 2001). The position of the fossa differs in osteichthyans, placoderms, and Acanthodes (Miles, 1964, 1973; Jarvik, 1977, 1980; Schaeffer, 1981; Gardiner, 1984a; Goujet, 1984), but it usually lies on or adjacent to the inferred position of the lateral commissure. Gardiner (1984a) considered this to be the primitive position for gnathostomes generally and that its posterior position in elasmobranchs is derived.

A classical distinction between osteichthyans and neoselachians involves the position of the hyomandibula relative to the lateral head vein; the articulation is ventromedial to the vein in elasmobranchs and lateral to it in osteichthyans (De Beer, 1937). Although its phylogenetic significance is unclear, this difference is a highly conserved trait in both groups. Gardiner (1984a: 241) argued that this difference is not significant on the grounds that the fossa lay above the vein in some extinct sharks such as Hybodus and Tristychius. In Hybodus and Tribodus, however, the lateral head vein probably passed above the hyomandibula, as in neoselachians.

OCCIPITAl ARCh, OtICO-OCCIPITAl FisSURE, AND HYPOTIC LAMINA: The occipital pila in gnathostomes arises during ontogeny behind the otic capsules, from which it is initially separated by the otico-occipital fissure (e.g., Scyliorhinus; De Beer, 1931, 1937: Squalus; Holmgren, 1940; El-Toubi, 1949). De Beer (1931) found that the glossopharyngeal nerve in Scyliorhinus lies within the metotic fissure (a transient embryonic space between the floor of the otic capsule and the hypotic lamina; fig. 29). He demonstrated that ontogenetic obliteration of the fissure leaves only the glossopharyngeal canal in adult neoselachians. This closure may occur earlier in some taxa than in others; for example, there are indications of a persistent fissure in the 62-mm Heterodontus figured by Holmgren (1940: fig. 122). In all adult neoselachians, the occipital arch is fused to the otic region. An embryonic otico-occipital fissure was presumably also present in hybodonts (e.g., Hybodus, Tribodus), where the adult occipital arch is continuous with the otic region as in neoselachians.

In many neoselachians, the occipital arch is wedged almost completely between the otic capsules (e.g., Chlamydoselachus, Heptranchias, Squalus; Allis, 1923a; Holmgren, 1941; Schaeffer, 1981), but in others it may extend behind the otic region (e.g., Notorynchus; Daniel, 1934; Maisey, 2004b). Where the occipital arch is short, all the spino-oc- 
cipital nerves may be confined to the glossopharyngeal canal, but where it is longer the more posterior nerves tend to emerge onto the lateral surface of the occipital region, although the anterior ones may still enter the glossopharyngeal canal.

Schaeffer (1981) considered that persistence of an otico-occipital fissure is an apomorphic character of adult Orthacanthus and Tamiobatis. However, the fissure has subsequently been identified in several other $\mathrm{Pa}-$ leozoic chondrichthyans, including Cladodoides, Akmonistion, Cladodus elegans, "Cobelodus", and Pucapampella (Coates and Sequeira, 1998, 2001a, 2001b; Maisey, 2001a, 2001b, 2004a; Maisey and Anderson, 2001).

The otico-occipital fissure in Orthacanthus consists of two distinct but interconnected regions, one located dorsally, between the occipital arch and otic capsules (regarded here as the otico-occipital fissure proper), and the other lying below the capsule and above the hypotic lamina (the metotic fissure). The extent of the metotic fissure seems to be governed by the degree to which the hypotic lamina becomes secondarily fused to the floor of the otic capsule during ontogeny. In Orthacanthus, the metotic fissure is extensive and forms a deep ventral otic notch (Schaeffer, 1981: figs. 5, 6), whereas in Cladodoides, the metotic fissure is mostly closed and there is no ventral otic notch (figs. 5, 6, $8 \mathrm{~B}, 21,27)$, although in both taxa the dorsal part of the fissure is persistent. In neoselachians and hybodonts, part of the metotic fissure can persist as a glossopharyngeal canal, following closure of the dorsal part of the fissure (De Beer, 1937; Schaeffer, 1981). Moreover, in many neoselachians, the occipital pila is typically fused to the otic capsule before the arch is complete dorsally (Holmgren, 1940; El-Toubi, 1949), whereas in extinct sharks such as Cladodoides the fissure remained open dorsally even after the metotic fissure became closed and the occipital arch was complete. However, in 62-mm Heterodontus, a complete occipital arch is present and a fissure continues to divide the pila from the otic capsule laterally, although the arch is connected to the synotic tectum dorsally, via the medial tectum forming the floor of the parietal fossa (Holmgren, 1940: 174; also see fig. 38). The fissure is never completely "lost" in elasmobranchs, since it is represented at least transiently in the embryo, although its extent is often reduced following ontogenetic fusion of the otic capsule to the occipital pila dorsally and to the hypotic lamina ventrally.

The otico-occipital fissure is similar in early elasmobranchs and osteichthyans, suggesting that it is a conserved feature of crown-group gnathostomes. Furthermore, in both groups it may be connected with a persistent ventral otic fissure ventrolaterally, separating the parachordal plate from the rest of the basicranium (for osteichthyans see Rayner, 1951; Nielsen, 1942; Patterson, 1975; Maisey, 1999; Basden et al., 2000; Basden and Young, 2001: for chondrichthyans see Maisey, 2001b; Maisey and Anderson, 2001). Nielsen (1942) and Patterson (1975) recognized that the otico-occipital and ventral otic fissures of osteichthyans differ from each other developmentally, morphologically, and evolutionarily, and this may also be true for chondrichthyans (Maisey, 2001b; Maisey and Anderson, 2001).

Young (1986) suggested that the glossopharyngeal nerve in gnathostomes is primitively located beneath the otic capsule (a view first advocated by De Beer, 1937) and that its passage through the capsule is a derived feature of arthrodires and osteichthyans (most parsimoniously regarded as an independently developed feature instead of a synapomorphy). The glossopharyngeal nerve also ran through the capsule in osteostracans, although this is probably because all the visceromotor cranial nerves had a strongly anterolateral orientation resulting from the considerable forward shifting of the gill compartments relative to the brain (Janvier, 1981b, 1985). Presence of a hypotic lamina might also represent a primitive gnathostome character, since Young (1986: 42) observed that in many placoderms (e.g., acanthocoracids, rhenanids, and petalichthyids) the parachordal plate is expanded beneath the otic capsule, effectively forming a hypotic lamina beneath the glossopharyngeal nerve as in elasmobranchs. However, he also noted that in arthrodires the glossopharyngeal nerve apparently passed through the labyrinth cavity (e.g., Buchanosteus; Young, 1979). Con- 
versely, Schaeffer (1981) concluded that the hypotic lamina is a derived feature of elasmobranchs and implied that its ontogenetic fusion to the capsular floor (to form the glossopharyngeal canal) is a further derived feature of neoselachians (see also Maisey, 1984a, 2001b; Coates and Sequeira, 1998; Maisey and Anderson, 2001). This conflicts with Young's (1986) contention that the glossopharyngeal nerve is primitively enclosed by a canal in gnathostomes, but not with De Beer's (1937) more general observation regarding the position of the nerve relative to the otic capsule. An enclosed glossopharyngeal canal may therefore have been acquired independently by arthrodires and elasmobranchs.

The hypotic lamina (= "lateral pharyngohyal plate" of Jollie, 1971) in neoselachians is a lateral extension of the basal (parachordal) plate that secondarily fuses with the otic capsule at the posterior basicapsular commissure. In osteichthyans and chimaeroids, a hypotic lamina is absent and the glossopharyngeal nerve typically leaves the braincase ventrally rather than ventrolaterally. Gardiner (1984a: 204) considered that the ventral otic notch in Orthacanthus and Tamiobatis vetustus is homologous to the vestibular fontanelle of osteichthyans. However, the osteichthyan vestibular fontanelle represents only one part of the primitive gnathostome metotic fissure and corresponds to the embryonic basicapsular fenestra (which may remain open even after the rest of the metotic fissure is closed by the posterior basicapsular commissure; De Beer, 1937). In osteichthyans, the vestibular fontanelle is situated anterior or lateral to the glossopharyngeal foramen (Gardiner, 1984a), but the metotic fissure in elasmobranchs extends farther laterally because the parachordal plate is broadened to form the hypotic lamina. Since the metotic fissure remains completely open in Orthacanthus and "Tamiobatis sp.", a posterior basicapsular commissure was presumably absent in these forms. In Cladodoides, Hybodus, Tribodus, and neoselachians, fusion of the hypotic lamina to the capsular floor by the commissure does not leave a persistent vestibular fontanelle like that of osteichthyans.

To summarize, several different conditions may be recognized in the gnathostome oticooccipital fissure: (1) hypotic lamina absent: (a) dorsal and metotic parts of the fissure open, including a posterior dorsal fontanelle (some early osteichthyans and perhaps Acanthodes); (b) dorsal and metotic parts of the fissure completely closed; no vestibular fontanelle (chimaeroids, some osteichthyans, arthrodires); (c) fissure closed apart from the vestibular fontanelle (some osteichthyans). (2) hypotic lamina present; (a) dorsal and metotic parts of the fissure open, medial tectum absent (Pucapampella, Orthacanthus); (b) metotic part of the fissure closed apart from glossopharyngeal canal; dorsal part open, medial tectum present (Cladodoides, "Cobelodus", Tamiobatis vetustus); (c) fissure completely closed except for glossopharyngeal canal and parietal fossa (neoselachians, Hybodus, Tribodus); (d) fissure completely closed except for glossopharyngeal canal; no posterior dorsal fontanelle (placoderms).

In modern chondrichthyans and osteichthyans, the cartilage containing the posterior semicircular canal and its ampulla also forms the outer wall of the otico-occipital fissure. By contrast, there is no occipital segment in modern agnathans, and the otic capsule defines the posterior limit of the braincase (Janvier, 1996). Thus, the otico-occipital fissure in crown-group gnathostomes effectively marks the posterior boundary of the primitive craniate braincase (in lampreys, the glossopharyngeal and vagal cranial nerves lie behind the otic capsule). However, in both placoderms and osteostracans, the glossopharyngeal, vagal, and anteriormost spino-occipital nerves are enclosed by the occipital region. In placoderms, the occipital region is often very extensive and may include numerous spino-occipital nerves (e.g., Buchanosteus). The braincase of placoderms and osteostracans therefore extends farther posteriorly than in modern cyclostomes, although there is no evidence of an otico-occipital fissure as in crown-group gnathostomes. If osteostracans and placoderms are successive sister groups to crown-group gnathostomes (as suggested by modern phylogenetic hypotheses; e.g., Janvier, 1996), incorporation of the occipital region into the braincase is most parsimoniously 
interpreted as a primitive conserved feature of crown-group gnathostomes.

In craniate embryos, the notochord usually extends beyond the occipital region and is flanked by the parachordal cartilages, although some variation is noted in the relative positions of the parachordals and notochord in gnathostomes (De Beer, 1937: 379). The notochord may be closely associated with the parachordals throughout their length (e.g., urodeles, anurans, mammals) or it may diverge anteriorly to leave a basicranial fenestra (e.g., neoselachians, osteichthyans, nonmammalian amniotes). In modern cyclostomes, chondrichthyans, sturgeons, and lungfishes the notochord is usually persistent into the adult. In other gnathostomes, it may become reduced to the posterior part of the parachordal plate (e.g., Polypterus), partly chondrified by notochordal cartilage (e.g., anurans, urodeles) or even obliterated (e.g., mammals). The parachordals are largely separated by the notochord in Amia and teleosts, but in neoselachians, Polypterus, and lungfishes, the parachordals fuse above and below the notochord, completely enclosing it. The parachordals fuse below the notochord in chimaeroids, Lepisosteus, urodeles, and lacertilians, leaving the notochord dorsal to the basal plate. In anurans, however, the reverse occurs and the notochord lies below the basal plate. The notochord is enclosed by cartilage apparently of parachordal derivation in placoderms (e.g., Kujdanowiaspis; Stensiö, 1963a: fig. 29), primitive actinopterygians (e.g., Mimia; Gardiner, 1984a: fig. 26), and primitive chondrichthyans (e.g., $P u$ capampella; Maisey and Anderson, 2001), suggesting that it represents a primitive gnathostome arrangement.

According to Miles (1973), in Acanthodes the ventral occipital ossification (also presumably of parachordal derivation) enclosed the notochord in a canal, and the dorsal aorta passed beneath the bone. However, Jarvik $(1977,1980)$ considered that the aorta lay inside the canal (implying that the notochord was positioned farther dorsally). Miles' (1973) interpretation is compromised by the presence of paired lateral canals arising from the central canal (said to house occipital arteries), suggesting that it contained a vessel rather than the notochord. According to Jar- vik $(1977,1980)$, the paired canals contained metameric arteries arising from the dorsal aorta. In either case, the ventral occipital ossification in Acanthodes presumably formed in the parachordal plate and the notochord terminated at or behind the ventral otic fissure.

What KIND OF SHARK WAS CLADODOIDES?: Schaeffer (1981) drew attention to similarities between the Cladodoides braincase and three specimens tentatively referred to Ctenacanthus from the Cleveland Shale, and he also noted their association with "cladodont" teeth. At present, there are four known or suspected associations of Cladodus-like teeth with Tamiobatis-like braincases: (1) Cladodoides wildungensis from Wildungen (Jaekel, 1921; Gross, 1937, 1938); (2) Ctenacanthus (compressus?) from the Cleveland Shale (Dean, 1909; Schaeffer, 1981); (3) "Tamiobatis vetustus" from the Cleveland Shale (Williams, 1998, although not demonstrably referable to Tamiobatis); and (4) Cladodus elegans from the Lower Carboniferous of Scotland (in prep.).

Ctenacanthus-like finspines (in the restricted sense outlined by Maisey, 1981) are associated with both taxa from the Cleveland Shale (Dean, 1909; Williams, 1998). Cladodont teeth are also present in "Ctenacanthus" costellatus (Lower Carboniferous of Scotland; Traquair, 1884), although its braincase is unknown and its finspine ornament differs from that of Ctenacanthus. Cladodus elegans may have possessed large finspines like those of Ctenacanthus major, but at present this cannot be confirmed. There is no proven association between the holotype of Cladodoides wildungensis (jaw cartilages associated with teeth) and the Senckenberg braincase. It is also unknown whether this taxon possessed finspines, and the only reported finspine from Wildungen (Ctenacanthus jaekeli Gross, 1933) is not typical of the genus and was subsequently referred to Homacanthus (thought to be an acanthodian; Denison, 1979); unfortunately, this specimen is now lost (Maisey, 1984c). Paleozoic sharks with a Tamiobatis-like braincase, Cladodus teeth, and Ctenacanthus finspines may be characterized loosely as a "ctenacanth" gestalt, which is also represented by numerous disarticulated fossils (probably including 
much of the material classically referred to Ctenacanthus, Tamiobatis, and Cladodus). However, it is uncertain whether this gestalt represents a monophyletic group of elasmobranchs or a paraphyletic assemblage of phalacanthous sharks that also includes "higher" forms such as hybodonts and neoselachians.

Do the Senckenberg and the Humboldt University braincases represent a single taxon? This question cannot be adequately addressed until the Humboldt Museum specimen is thoroughly reinvestigated. At present, all that can be said is that all features in the ventral part of the orbit agree closely and that many features of the basicranium also agree (with the proviso that the two individuals may have different ontogenetic ages). The major difference concerns labyrinth morphology, since its arrangement in the Humboldt University braincase as described by Stensiö (1937) is completely at odds with observations of the scanned Senckenberg example.

Phylogenetic Patterns in Paleozoic SHARKS: When Gross (1937) first described the Cladodoides braincase, elasmobranch phylogeny was still very much in its infancy. (It was the first Paleozoic shark braincase ever described, and would remain the only one for almost three decades.) He left its systematic position unresolved beyond a general statement that it was more closely related to modern elasmobranchs than to holocephalans or acanthodians. Holmgren (1941: 79) made far more specific proposals and considered that modern Chlamydoselachus may be "a descendant of a Cladodus-like stock". Such a view is incompatible with modern phylogenetic analyses in which neoselachians including Chlamydoselachus are resolved as a monophyletic group that is only distantly related to most Paleozoic sharks (Shirai, 1982; Maisey, 1984a, 1984b; Maisey et al., 2004).

Romer (1964: 104) suggested that " $\mathrm{Cla}$ dodus", Tamiobatis, and xenacanths all possessed "a truly primitive elasmobranch type of braincase, characteristic of ancestral shark types in the Devonian', which was distinguished by having a relatively long otico-occipital region. He proposed that the elongate otico-occipital region of forms such as Tamiobatis and Orthacanthus represented a prim- itive feature for gnathostomes generally and noted a similar arrangement in many placoderms and early rhipidistians. He concluded that there has been a parallel evolutionary trend toward relative reduction of the oticooccipital region in "higher" fishes and tetrapods.

Schaeffer (1981) reached an opposite conclusion and proposed that the elongate oticooccipital region in the Paleozoic sharks he investigated represents a synapomorphy of a monophyletic group of early elasmobranchs that also supposedly included Ctenacanthus (although "ctenacanths" have not been characterized by any unique features, so their monophyly has not been demonstrated, and Ctenacanthus itself is founded on isolated fin spines). Coates and Sequeira (1998) were unable to retrieve Schaeffer's (1981) grouping in their cladistic analysis of 23 cranial characters, although they found a large monophyletic clade comprising $C$. wildungensis, Tamiobatis, Xenacanthus, Cladoselache, Hybodus and Tristychius (presumably this clade would also include crown-group elasmobranchs although these were not specifically part of their analysis).

Schaeffer (1981) united Orthacanthus and Tamiobatis on the basis of a single supposedly derived character (presence of an oticooccipital fissure). The fissure is now known in several additional Paleozoic elasmobranchs, including Akmonistion, Cladodoides, Pucapampella, and "Cobelodus" (Coates and Sequeira, 1998, 2001a; Maisey, 2001c, 2004a) and is clearly more widespread than Schaeffer (1981) suspected, although Coates and Sequeira (1998) still maintained that it could be a derived character in elasmobranchs generally. However, this seems unlikely since an otico-occipital fissure is present in modern Polypterus, gars, and Amia (filled by cartilage; Patterson 1975), and in primitive actinopterygians (e.g., Pteronisculus, Kentuckia, Mimia, Moythomasia, Ligulalepis; Nielsen, 1942; Rayner, 1951; Gardiner, 1984a; Basden and Young, 2001), primitive sarcopterygians (e.g., Eusthenopteron, Diabolepis, Youngolepis; Jarvik, 1980; Chang, 1995), and Acanthodes (Watson, 1937; Miles, 1965, 1973; Jarvik, 1977; Gardiner, 1984a). The fissure is closed in modern chondrichthyans, Latimer- 
ia, dipnoans, and tetrapods (Gardiner, 1984a; Forey, 1998), and it is absent in all placoderms, osteostracans, and galeaspids where the braincase is known. An otico-occipital fissure therefore seems to be restricted to crown-group gnathostomes (Maisey, 2001c), and its presence is clearly apomorphic among craniates generally, whether it represents a synapomorphy of crown-group gnathostomes or appeared independently in several gnathostome lineages.

Schaeffer (1981) united Cladodoides, Tamiobatis, and Orthacanthus by one character (otico-occipital region equal to or longer than ethmo-orbital region). Unfortunately, the length of the ethmo-orbital region cannot be accurately determined in Cladodoides, but its otic region is certainly longer than the orbit (although less so than in Tamiobatis and Orthacanthus). In Coates and Sequeira's (1998) analysis, this character helped support a node including all these taxa plus Hybodus and Tristychius, but it was reversed at the node including the last two taxa. Thus, the feature may characterize a group of Paleozoic sharks, but there is uncertainty whether this group is monophyletic. A long otic region is also present in other Paleozoic phalacanthous sharks that may be closely related to Cladodoides, Tamiobatis, and/or Orthacanthus but which were not included in Schaeffer's (1981) analysis (e.g., Goodrichthys, Ctenacanthus). The otic region is also quite long in Wodnika (which may be a primitive neoselachian; Maisey, 1984a).

Schaeffer (1981) united Ctenacanthus with Cladodoides, Tamiobatis and Orthacanthus by two additional characters, but in the Cladodoides braincase one of these (multilayered prismatic calcification) is absent and the other character is unknown (lateral otic process "pronounced"). Thus, the similarities he noted are only applicable to cranial morphology in Cleveland Shale "ctenacanths," Tamiobatis, and xenacanths.

In Orthacanthus, the ventral otic notch (representing a persistent metotic fissure) is very long, but the notch is considerably shorter in Cladodoides, Tamiobatis vetustus (NMNH 1717), and "Tamiobatis sp." (AMNH 2140). Closure of the fissure (as in neoselachians and hybodonts) may be a derived character, but the elongated fissure in
Orthacanthus could be an apomorphy of some (or even all) xenacanths. Alternatively, if the glossopharyngeal canal is a primitive gnathostome character as Young (1986) suggested, a persistent metotic fissure may be a synapomorphy of some elasmobranchs (although this seems doubtful in view of the situation in Pucapampella, which is apparently a cladistically primitive chondrichthyan; Maisey, 2001b; Maisey and Anderson, 2001).

In Coates and Sequeira's (1998) phylogenetic analysis, symmoriids and stethacanthids (represented by Cobelodus aculeatus and Akmonistion zangerli) consistently fall outside a group comprising xenacanths, Tamiobatis, Cladodoides, Hybodus, and Tristychius. However, no cranial synapomorphies were identified for Cobelodus and Akmonistion, and these taxa were not resolved as a monophyletic group. Coates and Sequeira (1998) nevertheless recognized a large monophyletic group of elasmobranchs including "Tamiobatis sp.", T. vetustus, Cladodoides wildungensis, Hybodus, and Tristychius (and, by inference, modern neoselachians, although these were not specified in their analysis). This group was characterized by several derived features, including: dorsal aorta dividing into paired lateral aortae behind the occiput; a single internal carotid foramen; otico-occipital length equal to or greater than ethmo-orbital region; slot-shaped endolymphatic fossa; anteroposteriorly expanded postorbital process; "ethmoid" (= orbital) articulation extends anteriorly from the level of the optic foramen, with a short suborbital shelf (note however that this is not the condition in orbitostylic sharks); and presence of a precerebral fontanelle.

The concept of an ancient group of cladodont sharks is entrenched as far back as the late 19th century, although earlier diagnoses are unsatisfactory. Woodward (1889: 16) summarized the Cladodontida as "an undiagnosable family, apparently closely allied to the Pleuracanthidae", noting that the pectoral fin was apparently "a uniserial archipterygium-intermediate between the truly biserial one of Pleuracanthus and the pectoral fin of modern sharks". Blot (1969) formally diagnosed the Cladodontidae (including Cladodus) by the presence of a long, 
straight pectoral metapterygial axis, although this is unknown in C. mirabilis and $C$. wildungensis. Miles (in Moy-Thomas and Miles, 1971) also grouped Paleozoic sharks with such an axis in the order Cladodontida and again included $C$. wildungensis despite the lack of postcranial data. Both classifications imply that a close relationship exists between $C$. wildungensis, stethacanthids, and symmoriids, which is not supported by subsequent investigations (Schaeffer, 1981; Maisey, 1984a; Coates and Sequeira, 1998), and the classical view that "cladodont" sharks form a monophyletic group is generally considered obsolete. However, if the tribasal pectoral endoskeleton (with anterior basals homologous to the propterygium and mesopterygium plus a metapterygium) is the primitive condition for gnathostomes (Janvier, 1996), the condition represented in extinct sharks such as Cladoselache, symmoriids, and stethacanthids (with a series of premetapterygial radials plus a segmented metapterygial "axis") may represent a derived condition. Cladodoides, "Cobelodus", and "Tamiobatis sp" are united by an extensive polar cartilage-derived region, anterior position of the orbital articulation within the orbit, inclined orientation of the postorbital process, and exclusion of the trigeminal and facial foramina from the orbit. These features may eventually be recognized in other "cladodont" sharks, and it is interesting that all Paleozoic sharks implicated as possessing an extensive polar cartilage contribution probably had cladodont teeth. It is even conceivable that cladodont sharks really do form a monophyletic group (an old idea that has long been out of favor), characterized by apomorphic dental, cranial, and postcranial features, although it would be rash to present this in a formal classification based on the available data. Of particular interest is the fact that these apomorphic characters cut across other groupings that have been proposed in the past; for example, an extensive polar cartilage contribution is suggested in "Tamiobatis" sp. and Cladodoides, both of which have a "long" otic region, as well as in "Cobelodus", where the otic region is "short", but not in Orthacanthus, where the otic region is "long". The phylogenetic significance of the extensive polar cartilage in chimaeroids (in which the otic region can also be characterized as "short") is unresolved.

\section{CONCLUSIONS}

1. The original description of cranial morphology in Cladodoides presented by Gross (1937) is essentially accurate, and very few points of disagreement have been found (e.g., the position of the abducent foramen and the trigeminal mandibular ramus). Scanning has provided new information about the internal morphology of the braincase, especially the arrangement of cranial nerves, blood vessels, and the skeletal labyrinth. These new data mean that many aspects of cranial morphology in other extinct elasmobranchs such as Tamiobatis vetustus and Orthacanthus can now be interpreted with greater confidence.

2. The braincase is probably from a juvenile or submature individual. It shows indications of incomplete development (e.g., the presence of a wide bucco-hypophyseal fenestra and absence of a precarotid commissure; incomplete enclosure of the internal carotids and orbital arteries by basicranial cartilage; incomplete closure of the braincase roof along the dorsal midline). However, only features which develop relatively late in neoselachian ontogeny are implicated, suggesting that cranial development was essentially complete. In addition, the braincase lacks multilayered prismatic calcification (cf. Schaeffer, 1981), which may represent a further juvenile condition.

3. The efferent pseudobranchial and internal carotid arteries meet above the trabeculae in neoselachians, hybodonts, and many Paleozoic sharks, including Cladodoides, Tamiobatis and Orthacanthus. This probably represents a derived condition, in contrast with osteichthyans, placoderms, Acanthodes, and primitive chondrichthyans such as Pucapampella. The efferent pseudobranchial arrangement in modern chimaeroids is also considered to be derived and may have arisen from a condition similar to that found in Pucapampella.

4. A "basal" (i.e., palatobasal) articulation between the palatoquadrate and cranium is present in crown-group gnathostomes (osteichthyans plus chondrichthyans), Acanthodes, and perhaps in placoderms. The orbital articulation in neoselachians is probably homologous to the palatobasal articulation found in osteichthyans and early chondrichthyans such as Pucapampella. However, the articulation in orbitostylic neoselachians clearly repre- 
sents an apomorphic condition. A palatobasal articulation is inferred to be present in Paleozoic chondrichthyans such as Cladodoides, based on its topographic relationship to that region of the basicranium inferred to be derived from the embryonic polar cartilage. However, this area extends anteriorly within the orbit (probably representing an apomorphic condition). In Heterodontus and modern galeomorphs, an ethmoidal articulation is developed on the trabecular cartilage anteriorly, but this is unrelated ontogenetically to the polar cartilage and here is not considered an orbital articulation. An ethmoidal articulation seems to be present in extinct Mesozoic sharks such as Synechodus and $\mathrm{Hy}$ bodus, as well as in much earlier chondrichthyans such as Pucapampella and Debeerius. The palatobasal and ethmoidal articulations are presumably obscured by fusion of the palatoquadrate to the braincase in modern chimaeroids.

5. A very large area of the basicranium was probably derived from the polar cartilages in Cladodoides, since: (1) two landmark features marking the anterior and posterior margins of the polar cartilage in neoselachians (the efferent pseudobranchial and pituitary vein foramina) are widely separated; (2) the internal carotid grooves continue around the lateral walls of the bucco-hypophyseal fenestra, suggesting that the postpituitary commissure (formed by the polar cartilage in neoselachians) may also have extended around the fenestra; and (3) the dorsum sellae and the hypophyseal chamber (closely associated with the polar cartilage in neoselachians) are both very extensive. The polar cartilage probably also made an extensive contribution to the basicranium in Tamiobatis, "Cobelodus", and perhaps Akmonistion, but not in Orthacanthus. In modern elasmobranchs and osteichthyans, the polar cartilage is small (or even unrecognizable) and at best forms only a minor part to the basicranium, although it contributes to the hypophyseal chamber, dorsum sellae, and basal angle. In batoids, the embryonic polar cartilage is small but the dorsum sellae and hypophyseal chamber are reduced or absent. Reconstructions showing a large polar cartilage-derived region in placoderms (e.g., Stensiö, 1963a) may be erroneous, since the inferred position of the efferent pseudobranchial artery is probably incorrect and it probably lay farther posteriorly (Young, 1986).

6. In chondrichthyans, a broad suborbital shelf may have been present primitively, anterior to the palatobasal articulation (e.g., Pucapampella). Presence of an extensive suborbital shelf behind this articulation may be a derived feature of some Paleozoic sharks such as Cladodoides. It is uncertain whether the extensive suborbital shelf in modern galeomorph sharks is primitive or if it represents an independently derived feature that appeared following the loss of a palatobasal articulation. Absence of a shelf anterior to the articulation may be a derived feature of orbitostylic sharks and of Paleozoic taxa such as Cladodoides, but it is unclear whether this represents a synapomorphy of a larger elasmobranch group.

7. The mesencephalic chamber is comparatively longer in Cladodoides and Orthacanthus than in neoselachians such as Squalus and Notorynchus and in hybodonts (e.g., Hybodus, Tribodus). The differing positions of the hypophyseal chamber and oculomotor nerve (relative to the otic capsule and postorbital process) in these forms may be related to the differing proportions of the mesencephalic chamber. However, the length of this chamber is apparently unrelated to the extent of the polar cartilage, since the chamber is long in both Cladodoides and Orthacanthus, but the inferred polar cartilage-derived region is far less extensive in Orthacanthus. The shorter mesencephalic chamber of hybodonts and neoselachians may represent an apomorphic condition.

8. In Cladodoides, there is a small ventral fossa containing the pituitary and abducent foramina as well as a pit for the external rectus muscle insertion. The fossa does not include either the trigeminal or facial foramina and therefore does not correspond exactly to the trigemino-facial recess in neoselachians. Two mutually exclusive phylogenetic hypotheses are suggested: the arrangement found in $\mathrm{Cla}$ dodoides may represent the plesiomorphic arrangement for elasmobranchs (in which case the trigemino-facial recess arose independently in modern elasmobranchs and early actinopterygians); alternatively, if the trigemino-facial recess is a conserved feature of crown-group gnathostomes (Gardiner, 1984a), the arrangement in Cladodoides may be derived. The facial foramen is located behind the orbit in some (perhaps all) sharks with an extensive polar cartilage, and these features may be related developmentally.

9. The postorbital arcade in Cladodoides is inclined anterodorsally. Consequently, its dorsal and ventral attachments do not lie at the same level on the lateral surface of the brain- 
case, the dorsal part of the process is anterior to the otic capsule, and the trigeminal and facial trunks left the braincase behind the postorbital process (as did the anterodorsal lateral line nerve). Presumably, the orientation of the embryonic lateral commissure in Cladodoides affected its relationship to the facial and trigeminal nerves. This may represent an apomorphic feature in Cladodoides.

10. The mandibular ramus of the trigeminal nerve probably passed through the postorbital process, unlike in neoselachians (where it passes anterior to the process), and also unlike Acanthodes (where it passed posterior to the process). The superficial ophthalmic ramus of the anterodorsal lateral line nerve in Cladodoides probably passed through the postorbital process dorsally before entering the orbit, while its buccal branch either passed anteriorly via the jugular canal or else accompanied the trigeminal nerve through the postorbital process (considered here the most plausible arrangement). The palatine ramus of the facial nerve in Cladodoides divided into anterior and posterior ramules before it left the braincase, and both ramules passed separately through the floor of the lateral commissure. Internally, the prefacial commissure forms an acustico-trigemino-facial recess and isolates the facial nerve proper from the trigeminal and anterodorsal lateralis trunks, as in gnathostomes generally.

11. The large dorsal opening between the otic capsules of Cladodoides is identified as a posterior dorsal fontanelle, not a parietal (endolymphatic) fossa. In primitive chondrichthyans, this fontanelle does not form part of a low-frequency phonoreceptor system like the parietal fontanelle of neoselachians. A posterior dorsal fontanelle is also present in Acanthodes, early actinopterygians, modern chondrosteans (e.g., Acipenser), and in early sarcopterygians (e.g., Eusthenopteron). Presence of the fontanelle is here regarded as a synapomorphy of crown-group gnathostomes. Primitively, this fontanelle is connected to the dorsal part of the otico-occipital fissure. In placoderms and ostracoderms both the fontanelle and fissure are absent (also see below).

12. The otic capsule of Cladodoides primitively resembles that of Acanthodes, osteichthyans, and chimaeroids in several respects: a crus commune is retained, the posterior semicircular canal is not isolated, the medial capsular wall is unchondrified, and there is no evidence of perilymphatic fenestrae. In Cladodoides and Mimia, the cranial and labyrinth spaces are similarly separated anteriorly by a septum ending just above an unchondrified acustico-trigemino-facial fossa. The latter represents a conserved feature of crowngroup gnathostomes. Similarities noted by earlier investigators in the inner ear of neoselachians and arthrodires probably represent a mixture of primitive (craniate, gnathostome) and convergent features.

13. Labyrinth morphology in Cladodoides differs profoundly from that described by Stensiö (1937) in the Humboldt University "Cladodus" specimen, in which the semicircular canals are purportedly arranged as in neoselachians. Further investigation of the Humboldt University specimen is required in order to establish the validity of Stensiö's interpretation. If Stensiö's claims are substantiated, "Cladodus" hassiacus and Cladodoides wildungensis are very different taxa.

14. The hyomandibular fossa is not preserved in the Cladodoides braincase but was probably located on the posterolateral wall of the otic capsule, as in Orthacanthus. The position of the fossa in elasmobranchs is probably apomorphic.

15. The basicranial arteries in Tamiobatis vetustus were probably arranged as in Cladodoides wildungensis, and many discrepancies in the earlier literature can now be explained. Paired lateral aortae are present in many early chondrichthyans and sarcopterygians. A median aortic canal is present in Acanthodes, actinopterygians, early holocephalans (e.g., Helodus; Moy-Thomas, 1936), the stethacanthid shark Akmonistion (Coates and Sequeira, 1998, 2001b), and in "Cobelodus" (in prep.). Gardiner (1984a: 207) was undecided whether presence of a median aortic canal is a primitive gnathostome character or if it arose independently (as suggested by its phylogenetically restricted occurrence). Presence of a median aorta is probably apomorphic, but further investigation is necessary to determine whether it represents a synapomorphy of holocephalans and some Paleozoic sharks.

16. Persistence of the otico-occipital fissure is regarded here as a synapomorphy of crowngroup gnathostomes plus Acanthodes. The fissure corresponds to the posterior end of the braincase in hagfishes and lampreys, which lack an occipital region. An occipital region is well developed in placoderms and ostracoderms (suggesting that the modern cyclostome arrangement may be derived), but there is no evidence of an otico-occipital fissure. In Cladodoides, the ventral (metotic) part of the otico-occipital fissure (corresponding to the 
embryonic basicapsular fenestra) is closed and the hypotic lamina is fused to the overlying otic capsule, forming a glossopharyngeal canal as in neoselachians. However, the canal is continuous with the dorsal part of the otico-occipital fissure (containing the vagal nerve), which contradicts Gardiner's (1984a) contention that an actinopterygian-like vestibular fontanelle bounded by a posterior basicapsular fenestra is present in elasmobranchs. A vestibular fontanelle is absent in many placoderms and all osteostracans and galeaspids where the braincase is known, but is present in sarcopterygians (including tetrapods, where part of it forms the fenestra ovalis; Gardiner, 1984a: 204). However, in actinopterygians and sarcopterygians it represents a different part of the metotic fissure from the glossopharyngeal canal of neoselachians. Neither Gardiner's (1984a) claim that a vestibular fontanelle is present in Acanthodes, nor his conclusion that the fontanelle represents a conserved gnathostome condition (implying that its absence in sharks is secondary) can be confirmed.

17. A persistent ventral otic fissure at the junction of the parachordal and trabecular-polar cartilages is present in crown-group gnathostomes and probably in Acanthodes, but it is absent in cyclostomes, placoderms, and ostracoderms.

\section{ACKNOWLEDGMENTS}

I thank G. Plodowsky (Forschungsinstitut und Naturmuseum Senckenberg, Frankfurt) for permitting me to borrow the Cladodoides braincase for study. Timothy Rowe, Richard Ketchum, and Matthew Colbert (University of Texas at Austin) scanned the specimen and provided preliminary imaging. David Reddy (formerly at the American Museum of Natural History) gave invaluable help in imaging and visualization. Chester Tarka and Lorraine Meeker (AMNH) provided conventional photography and made the reproduction of Walter Gross' original illustrations. I also thank M. Ginter, G. Northcutt, B. Schaeffer, and the late M. Williams for many useful discussions about chondrichthyan and gnathostome morphology. The author is indebted to P. Janvier and M. Coates for their careful and informative reviews of this paper. This research was undertaken almost entirely at the American Museum of Natural History. Funding (including computer hardware, soft- ware licenses, and scanning expenses) was provided by the Herbert and Evelyn Axelrod Research Chair in Paleoichthyology (an endowed fund in the Division of Paleontology). Finally, I thank Maria da Gloria P. de Carvalho for her support during the preparation of this work.

\section{REFERENCES}

Agassiz, J.L.R. 1833-44. Recherches sur les poissons fossiles. 5 vols. Neuchatel: Imprimerie Petitpierre.

Allis, E.P. 1914. The pituitary fossa and trigemino-facialis chamber in selachians. Anatomischer Anzeiger 46: 225-253.

Allis, E.P. 1923a. The cranial anatomy of Chlamydoselachus anguineus. Acta Zoologica 4: 123-221.

Allis, E.P. 1923b. Are the polar and trabecular cartilages of vertebrate embryos the pharyngeal elements of mandibular and premandibular arches? Journal of Anatomy 58: 37-51.

Allis, E.P. 1928. Concerning the pituitary fossa, the myodome and the trigemino-facialis chamber in recent gnathostome fishes. Journal of Anatomy 63: 95-141.

Basden, A.M., G.C. Young, M. Coates, and A. Ritchie. 2000. The most primitive osteichthyan braincase? Nature 403: 185-188.

Basden, A.M., and G.C. Young. 2001. A primitive actinopterygian neurocranium from the early Devonian of southeastern Australia. Journal of Vertebrate Paleontology 21: 745-766.

Bertmar, G. 1959. On the ontogeny of the chondral skull in Characidae, with a discussion on the chondrocranial base and the visceral chondrocranium in fishes. Acta Zoologica (Stockholm) 40: 203-364.

Bjerring, H. 1978. The "intracranial joint" versus the "ventral otic fissure". Acta Zoologica (Stockholm) 59: 203-214.

Bleckman, H., and M.H. Hoffman. 1999. Special senses. In W.C. Hamlett (editor), Sharks, skates and rays; the biology of elasmobranch fishes: 300-328. Baltimore: Johns Hopkins University Press.

Blot, J. 1969. Systématique. In J. Pivetau (editor), Traité de paléontologie, IV (2), Gnathostomes, acanthodiens, placodermes, élasmobranches: 702-776. Paris: Masson.

Cerny, R., P. Lwigale, R. Ericsson, D. Meulemans, H.-H. Epperlein, and M. Bonner-Fraser. 2004. Developmental origins and evolution of jaws: new interpretation of "maxillary" and "mandibular". Developmental Biology 276: 225-236.

Chai, Y., X. Jiang, Y. Ito, P. Bringas, J. Han, D.H. 
Rowitch, P. Soriano, A.P. McMahon, and H.M. Sucov. 2000. Fate of the mammalian cranial neural crest during tooth and mandibular morphogenesis. Development 127: 1671-1679.

Chang, M.M. 1995. Diabolepis and its bearing on the relationships between porolepiforms and dipnoans. Bulletin du Muséum National d'Histoire Naturelle (Paris) 4e série 17: 235268.

Chibon, P. 1974. Un système morphogénétique remarquable; la crête neurale des vertébrés. Annales de Biologie 13: 459-480.

Chorn, J., and K.N. Whetstone. 1978. The use of the term nomen vanum in taxonomy. Journal of Paleontology 52: 494.

Coates, M.I., and S.E.K. Sequeira. 1998. The braincase of a primitive shark. Transactions of the Royal Society of Edinburgh Earth Sciences 89: 63-85.

Coates, M.I., and S.E.K. Sequeira. 2001a. Early sharks and primitive gnathostome interrelationships. In P.E. Ahlberg (editor), Major events in early vertebrate evolution: palaeontology, phylogeny, genetics and development: 241-262. New York: Taylor and Francis for the Systematics Association.

Coates, M.I., and S.E.K. Sequeira. 2001b. A new stethacanthid chondrichthyan from the Lower Carboniferous of Bearsden, Scotland. Journal of Vertebrate Paleontology 21: 438-459.

Cohn, M.J. 2002 Lamprey Hox genes and the origins of jaws. Nature 416: 386-387.

Corwin, J.T. 1989. Functional anatomy of the auditory system in sharks and rays. Journal of Experimental Zoology. 2(suppl.): 62-74.

Daget, J., and F. d'Aubenton. 1957. Développement et morphologie du crâne d'Heterotis niloticus Ehr. Bulletin de l'Institut Fondamental d'Afrique Noire 11: 1-178.

Daniel, J.F. 1934. The Elasmobranch fishes. Berkeley: University of California.

Dean, B. 1909. Studies on fossil fishes (sharks, chimaeroids and arthrodires). Memoirs of the American Museum of Natural History 9: 209287.

De Beer, G.R. 1924. Contributions to the study of the development of the head in Heterodontus. Quarterly Journal of Microscopical Science 68: 39-65.

De Beer, G.R. 1931. The development of the skull in Scyllium (Scyliorhinus) canicula L. Quarterly Journal of Microscopical Science, n. ser. 74: 591-652.

De Beer, G.R. 1937. The development of the vertebrate skull. Oxford: Clarendon Press.

De Beer, G.R., and J.A. Moy-Thomas. 1935. On the skull of Holocephali. Philosophical Trans- actions of the Royal Society of London (B) 224: 287-312.

Denison, R. 1979. Acanthodii. In H.-P. Schulze (editor), Handbook of paleoichthyology 5: 162. New York: Fischer.

Devillers, C. 1958. Le crâne des poissons. In P.P. Grassé (editor), Traité de zoologie 13(1): 551-687. Paris: Masson.

Dick, J.R.F. 1978. On the Carboniferous shark Tristychius arcuatus Agassiz from Scotland. Transactions of the Royal Society of Edinburgh 70: 63-109.

Didier, D. 1995. Phylogenetic systematics of extant chimaeroid fishes (Holocephali, Chimaeroidei). American Museum Novitates 3119: 1-86.

Eastman, C.R. 1897. Tamiobatis vetustus: a new form of fossil skate. American Journal of Science 4th. ser. 4: 85-90.

El-Toubi, M. R. 1949. The development of the chondrocranium of the spiny dogfish, Acanthias vulgaris (Squalus acanthias). Part 1. Neurocranium, mandibular and hyoid arches. Journal of Morphology 84: 227-280.

Forey, P. 1998. History of the coelacanth fishes. London: Chapman and Hall.

Francis-West, P., R. Ladher, A. Barlow, and A. Graveson. 1998. Signalling interactions during facial development. Mechanisms of Development 75: 3-28.

Gadow, H. 1888. On the modifications of the first and second visceral arches, with especial reference to the homologies of the auditory ossicles. Philosophical Transactions of the Royal Society of London (B) 179: 451-485.

Gardiner, B.G. 1984a. The relationships of the palaeoniscid fishes, a review based on new specimens of Mimia and Moythomasia from the Upper Devonian of Western Australia. Bulletin of the British Museum (Natural History) Geology 37(4): 173-428.

Gardiner, B.G. 1984b. The relationships of placoderms. Journal of Vertebrate Paleontology 4: 379-395.

Gaudin, T. 1991. A re-examination of elasmobranch monophyly and chondrichthyan phylogeny. Neues Jahrbuch für Geologie und Paläontologie Abhandlungen 182: 133-160.

Gegenbaur, C. 1872. Untersuchungen zur vergleichenden Anatomie der Wirbelthiere. III. Das Kopfskelet der Selachier, ein Beitrag zur Erkenntniss der Genese des Kopfskeletes der Wirbelthiere. Leipzig: Engelmann.

Ginter, M. 1998. Taxonomic problems with Carboniferous "cladodont-level" sharks. Ichthyolith Issues Special Publication 4: 14-16.

Ginter, M., and A. Ivanov. 1992. Devonian phoebodont shark teeth. Acta Palaeontologica Polonica 37: 55-75. 
Goodrich, E.S. 1930. Studies of the structure and development of vertebrates. London: Macmillan.

Goujet, D. 1984. Les poissons Placodermes du Spitzberg. Arthrodires Dolichothoraci de la Formation de Wood Bay (Dévonien Inferieur). Paris: Cahiers de Paléontologie, Centre National de la Recherche Scientifique.

Grogan, E.D., and R. Lund. 2000. Debeerius ellefseni (Fam. nov., gen. nov., spec. nov.), an autodiastylic chondrichthyan from the Mississippian Bear Gulch Limestone of Montana (USA), the relationships of Chondrichthyes, and comments on gnathostome evolution. Journal of Morphology 243: 219-245.

Gross, W. 1933. Die Wirbelthiere des Rheinischen Devons. Abhandlungen der Preussischen Geologischen Landesanstalt 154: 1-83.

Gross, W. 1937. Das Kopfskelett von Cladodus wildungensis Jaekel. 1. Teil. Endocranium und Palatoquadratum. Senckenbergiana 19: 80-107.

Gross, W. 1938. Das Kopfskelett von Cladodus wildungensis Jaekel. 2. Teil. Der Kieferbogen. Senckenbergiana 20: 123-145.

Haeckel, E.H.P.A. 1875. Die Gastrula und die Eifurchung der Tiere. Jenaische Zeitschrift für Naturwissenschaft 9: 402-508.

Hall, B.K. 1998. Evolutionary developmental biology, 2nd. ed. London: Chapman and Hall.

Hammarberg, F. 1937. Zur Kenntniss der ontogenetischen Entwicklung des Schädels von Lepidosteus platostomus. Acta Zoologica (Stockholm) 18: 209-337.

Heidtke, U.H.J. 1998. Revision der Gattung Orthacanthus Agassiz 1843 (Chondrichthyes: Xenacanthida). Paläontologische Zeitschrift 72: 135-147.

Heidtke, U.H.J. 1999. Orthacanthus (Lebachacanthus) senckenbergianus Fritsch 1889 (Xenacanthida: Chondrichthyes): revision, organisation und phylogenie. Freiburger Forschungsheft C481: 63-106.

Heidtke, U.H.J., and K. Krätschmer. 2001. Gladbachus adentatus nov. gen et sp., ein primitiver Hai aus dem Oberen Givetium (Obers Mitteldevon) der Bergische Gladbach-Paffrath-Mulde (Rheinisches Schiefergebirge). Mainzer Geowissenschaftliche Mitteilungen 30: 105-122.

Holmgren, N. 1940. Studies on the head in fishes. Part I. Development of the skull in sharks and rays. Acta Zoologica (Stockholm) 21: 51-267.

Holmgren, N. 1941. Studies on the head in fishes. Part II. Comparative anatomy of the adult selachian skull with remarks on the dorsal fins in sharks. Acta Zoologica (Stockholm) 22: 1-100.

Holmgren, N. 1942. Studies on the head in fishes. Part III. The phylogeny of elasmobranch fishes. Acta Zoologica (Stockholm) 23: 129-261.
Holmgren, N. 1943. Studies on the head in fishes. Part IV. General morphology of the head in fish. Acta Zoologica (Stockholm) 23: 1-188.

Hotton, N. 1952. Jaws and teeth of American xenacanth sharks. Journal of Paleontology 26: 489-500.

Hubendick, B. 1943. Zur Kenntnis der Entwicklung des Primordialcraniums bei Leusiscus rutilus. Arkiv für Zoologi 34(7): 1-35.

Huxley, T.H. 1875. Preliminary note on the brain and skull of Amphioxus lanceolatus. Proceedings of the Royal Society of London 23: 127132.

Huxley, T.H. 1876. Contributions to morphology. Ichthyopsida. No. 1. On Ceratodus forsteri, with observations on the classification of fishes. Proceedings of the Zoological Society of London 1876: 24-59.

Iselstöger, H. 1937. Das Neurokranium von Rhina squatina und einige Bemerkungen über ihre systematische Stellung. Zoologische Jahrbucher Abteilung für Anatomie und Ontogenie der Tiere 62: 349-394.

Jaekel, O. 1921. Die Stellung der Paläontologie zu einigen Problemen der Biologie und Phylogenie. Paläontologische Zeitschrift 3: 213-239.

Janvier, P. 1981a. The phylogeny of the Craniata, with particular reference to the significance of fossil "agnathans". Journal of Vertebrate Paleontology 1: 121-159.

Janvier, P. 1981b. Norselaspis glacialis n.g., n.sp. et les relations phylogénétiques entre les Kiaeraspidiens (Osteostraci) du Dévonien inférieur du Spitzberg. Palaeovertebrata 11(2, 3): 19131.

Janvier, P. 1984. The relationship of the Osteostraci and Galeaspida. Journal of Vertebrate Paleontology 4: 344-358.

Janvier, P. 1985. Les céphalaspides du Spitzberg. Cahiers de paléontologie. Paris: Centre National de la Recherche Scientifique.

Janvier, P. 1996. Early vertebrates. Oxford: Clarendon Press.

Janvier, P., and M. Suárez-Riglos. 1986. The Silurian and Devonian vertebrates of Bolivia. Bulletin de 1'Institut Français d'Etudes Andines 15(3, 4): 73-114.

Jarvik, E. 1954. On the visceral skeleton in Eusthenopteron with a discussion of the parasphenoid and palatoquadrate in fishes. Kungliga Svenska VetenskapsAkademiens Handlingar 5: 1-104.

Jarvik, E. 1977. The systematic position of acanthodian fishes. In S.M. Andrews, R.S. Miles, and A.D. Walker (editors), Problems in vertebrate evolution: 199-224. London: Academic Press. 
Jarvik, E. 1980. Basic structure and evolution of vertebrates. London: Academic Press.

Johnson, G.D. 1999. Dentitions of late Paleozoic Orthacanthus species and new species of? Xenacanthus (Chondrichthyes: Xenacanthiformes) from North America. Acta Geologica Polonica 49(3): 215-266.

Jollie, M. 1971. Some developmental aspects of the head skeleton of the 35-37 Squalus acanthias foetus. Journal of Morphology 133: $17-$ 40.

Jollie, M. 1962. Chordate morphology. London: Chapman and Hall.

Kuritani, S., Y. Murakami, Y. Nobusada, R. Kusakabe, and S. Hirano. 2004. Developmental fate of the mandibular mesoderm in the lamprey, Lethenteron japonicum: conservative morphology and development of the gnathostome jaw with special reference to the nature of the trabecula cranii. Journal of Experimental Zoology 302B: 458-468.

Lauder, G.V. 1980. On the evolution of the jaw adductor musculature in primitive gnathostome fishes. Breviora 460: 1-10.

Lund, R. 1985. The morphology of Falcatus falcatus (St. John and Worthen), a Mississippian stethacanthid chondrichthyan from the Bear Gulch Limestone of Montana. Journal of Vertebrate Paleontology 5: 1-19.

Luther, A. 1909. Untersuchungen über die vom N. trigeminus innervierte Muskulatur der Selachier (Haie und Rochen). Acta Societatis Scientiarum Fennicae 36: 1-168.

Maisch, M.W. 1998. Wildungenichthys grossi $\mathrm{n}$. gen. n. sp., a new selenosteid arthrodire (Placodermi, arthrodira) from the Kellwasserwerk (late Frasnian, Upper Devonian) of Bad Wildungen (Hessen, W. Germany). Palaeontologische Zeitschrift 72: 373-382.

Maisey, J.G. 1980. An evaluation of jaw suspension in sharks. American Museum Novitates 2706: 1-17.

Maisey, J.G. 1981. Studies on the Paleozoic selachian genus Ctenacanthus Agassiz. No. 1. Historical review and revised diagnosis of Ctenacanthus, with a list of referred taxa. American Museum Novitates 2718: 1-22.

Maisey, J.G. 1983. Cranial anatomy of Hybodus basanus Egerton from the Lower Cretaceous of England. American Museum Novitates 2758: $1-64$.

Maisey, J.G. 1984a. Chondrichthyan phylogeny: a look at the evidence. Journal of Vertebrate $\mathrm{Pa}$ leontology 4(3): 359-371.

Maisey, J.G. 1984b. Higher elasmobranch phylogeny and biostratigraphy. Zoological Journal of the Linnean Society 82: 33-54.

Maisey, J.G. 1984c. Studies on the Paleozoic se- lachian genus Ctenacanthus Agassiz. No. 3. Nominal species referred to Ctenacanthus. American Museum Novitates 2774: 1-20.

Maisey, J.G. 1985. Cranial morphology of the fossil elasmobranch Synechodus dubrisiensis. American Museum Novitates 2804: 1-28.

Maisey, J.G. 1987. Cranial anatomy of the Lower Jurassic shark Hybodus reticulatus (Chondrichthyes: Elasmobranchii), with comments on elasmobranch systematics. American Museum Novitates 2878: 1-39.

Maisey, J.G. 1999. The supraotic bone in neopterygian fishes (Osteichthyes, Actinopterygii). American Museum Novitates 3267: 1-52.

Maisey, J.G. 2001a. Remarks on the inner ear of elasmobranchs and its interpretation from skeletal labyrinth morphology. Journal of Morphology 250: 236-264.

Maisey, J.G. 2001b. A primitive chondrichthyan braincase from the Middle Devonian of Bolivia. In P.E. Ahlberg (editor), Major events in early vertebrate evolution: paleontology, phylogeny, genetics, and development: 263-288. New York: Taylor and Francis, for the Systematics Association.

Maisey, J.G. 2001c. CT-scan reveals new cranial features in Devonian chondrichthyan "Cladodus" wildungensis. Journal of Vertebrate Paleontology 21(4): 807-810.

Maisey, J.G. 2004a. Endocranial morphology in fossil and Recent chondrichthyans. In G. Arratia, M. Wilson, and $\mathrm{R}$. Cloutier (editors), Recent advances in the origin and radiation of early vertebrates. 139-170. Munich: Pfeil.

Maisey, J.G. 2004b. Morphology of the braincase in the broadnose sevengill shark Notorynchus (Elasmobranchii, Hexanchiformes), based on CT scanning. American Museum Novitates 3351: 1-52.

Maisey, J.G., and M.E. Anderson. 2001. A primitive chondrichthyan braincase from the early Devonian of South Africa. Journal of Vertebrate Paleontology 21(4): 702-713.

Maisey, J.G., and M.R. de Carvalho. 1997. A new look at old sharks. Nature 385: 779-780.

Maisey, J.G., G.J.P. Naylor, and D.J. Ward. 2004. Mesozoic elasmobranchs, neoselachian phylogeny and the rise of modern elasmobranch diversity. In G. Arratia and A. Tintori (editors), Mesozoic Fishes 3-Systematics, Paleoenvironments and Biodiversity. 17-56. Munich: Pfeil.

Marinelli, W., and A. Strenger. 1959. Vergleichende Anatomie und Morphologie der Wirbelthiere. III. Lieferung, pt. 2, Squalus acanthias: 179-308. Vienna: Franz Deuticke.

Meulemans, D., and M. Bonner-Fraser. 2002. Amphioxus and lamprey AP-2 genes: implications 
for neural crest evolution and migration patterns. Development 129: 4953-4962.

Miles, R.S. 1964. A reinterpretation of the visceral skeleton in Acanthodes. Nature 204: 457459.

Miles, R.S. 1965. Some features of the cranial morphology of acanthodians and the relationships of the Acanthodii. Acta Zoologica (Stockholm) 46: 233-255.

Miles, R.S. 1968. Jaw articulation and suspension in Acanthodes and their significance. Nobel Symposium 4: 109-127.

Miles, R.S. 1973. Relationships of acanthodians. In P.H. Greenwood, R.S. Miles, and C. Patterson (editors), Interrelationships of fishes: 63103. London: Academic Press.

Miller, R.F., R. Cloutier, and S. Turner. 2003. The oldest articulated chondrichthyan from the Early Devonian period. Nature 425: 501-504.

Moy-Thomas, J.A. 1936. On the structure and affinity of the Carboniferous cochliodont Helodus simplex. Geological Magazine 73: 488-503.

Moy-Thomas, J.A., and R.S. Miles. 1971. Palaeozoic fishes. Philadelphia: Saunders.

Nielsen, E. 1942. Studies on Triassic fishes from East Greenland. 1. Glaucolepis and Boreosomus. Meddelelser om Grønland 138: 1-403.

Norris, H.W., and S.P. Hughes. 1920. The cranial, occipital and anterior spinal nerves of the dogfish, Squalus acanthias. Journal of Comparative Neurology 31: 293-402.

Northcutt, R.G., and W.E. Bemis. 1993. Cranial nerves of the coelacanth Latimeria (Osteichthyes: Sarcopterygii: Actinistia) and comparisons with other Craniata. Brain, Behavior and Evolution 42(suppl. 1): 1-76.

Oelofsen, B.W. 1986. A fossil shark neurocranium from the Permo-Carboniferous (lowermost Ecca Formation) of South Africa. In T. Uyeno, R. Arai, T. Taniuchi, and K. Matsuura (editors), Indo-Pacific fish biology: Proceedings of the Second International Conference on Indo-Pacific Fishes: 107-124. Tokyo: Ichthyological Society of Japan.

P'an, K., and S.T. Wang. 1978. Devonian Agnatha and Pisces of South China. In Symposium on the Devonian System of South China: 299-333. Beijing: Geological Publishing House. [in Chinese]

Parker, T.J. 1887. Notes on Carcharodon rondeletii. Transactions of the Zoological Society of London 1887: 27-40.

Patterson, C. 1975. The braincase of pholidophorid and leptolepid fishes, with a review of the actinopterygian braincase. Philosophical Transactions of the Royal Society of London B269: 275-579.

Patterson, C. 1982. Morphological characters and homology. In K.A. Joysey, and A.E. Friday (editors), problems of phylogenetic reconstruction: 21-74. London: Academic Press.

Rayner, D.H. 1951. On the cranial structure of an early palaeoniscid, Kentuckia gen. nov. Transactions of the Royal Society of Edinburgh 62: 58-83.

Retzius, G. 1881. Das Gehörorgan der Wirbelthiere. Vol. 1. Stockholm: Samson and Wallin.

Romer, A.S. 1964. The braincase of the Palaeozoic shark Tamiobatis. Bulletin of the Museum of Comparative Zoology at Harvard University 131: 89-105.

Rosen, D.E., P.E. Forey, B.G. Gardiner, and C. Patterson. 1981. Lungfishes, tetrapods, paleontology and parsimony. Bulletin of the American Museum of Natural History 167: 159-276.

Schaeffer, B. 1967. Comments on elasmobranch evolution. In P.W. Gilbert, R.F. Mathewson, and D.P. Rall (editors), Sharks, skates and rays: 335. Baltimore: Johns Hopkins University Press. Schaeffer, B. 1971. The braincase of the holostean fish Macrepistius, with comments on neurocranial ossification in the Actinopterygii. American Museum Novitates 2459: 1-34.

Schaeffer, B. 1975. Comments on the origin and basic radiation of the gnathostome fishes with particular reference to the feeding mechanism. Colloque International Centre National de la Recherche Scientifique 218: 101-109.

Schaeffer, B. 1981. The xenacanth shark neurocranium, with comments on elasmobranch monophyly. Bulletin of the American Museum of Natural History 169: 3-66.

Sequeira, S.E.K., and M.I. Coates. 2000. Reassessment of "Cladodus" neilsoni Traquair: a primitive shark from the Lower Carboniferous of East Kilbride, Scotland. Palaeontology 43: 153-172.

Sewertzoff, A.N. 1899. Die Entwickelung des Selachierschädels. Ein Beitrag zur Theorie der korrelativen Entwickelung. Festschrift Carl von Kuppfer. Jena: Fischer.

Sewertzoff, A.N., and J. Disler. 1924. Das Pharyngomandibulare der Selachier. Anatomischer Anzeiger 58: 345-349.

Shirai, S. 1992. Squalean phylogeny: a new framework of "squaloid" sharks and related taxa. Sapporo: Hokkaido University Press.

Stensiö, E. 1927. The Downtonian and Devonian vertebrates of Spitzbergen, 1. Family Cephalaspidae. Skrifter om Svalbard og Nordishavet 12: $1-391$.

Stensiö, E. 1937. Notes on the endocranium of a Devonian Cladodus. Bulletin of the Geological Institute of Uppsala 27: 128-144.

Stensiö, E. 1950. La cavité labyrinthique, l'ossification sclérotique et l'orbite de Jagori- 
na. Colloques Internationaux du Centre National de la Recherche Scientifique 21: 9-1.

Stensiö, E. 1963a. Anatomical studies of the arthrodiran head. 1. Preface, geological and geographical distribution, the organisation of the arthrodires, the anatomy of the head in the Dolichothoraci, Coccosteomorphi and Pachyosteomorphi. Taxonomic appendix. Kungliga Svenska Vetenskapsakademiens Handlingar 9: 1419.

Stensiö, E. 1963b. The brain and the cranial nerves in fossil, lower craniate vertebrates. Skrifter av Det norske Videnskaps-Akademi i Oslo 1, N.S. 13: 5-120.

Stensiö, E. 1969. Elasmobranchiomorphi; Placodermata; Arthrodires. In J. Piveteau (editor), Traité de paléontologie 4(2): 71-92. Paris: Masson.

Summers, A.P. 2000. Stiffening the stingray skeleton-an investigation of durophagy in myliobatid stingrays (Chondrichthyes, Batoidea, Myliobatidae). Journal of Morphology 243: 113126.

Swinnerton, H.H. 1902. A contribution to the morphology of the teleostomian head skeleton, based upon a study of the developing skull in the three-spined stickleback (Gasterosteus aculeatus). Quarterly Journal of Microscopical Science 45: 503-593.

Torrey, T.W. 1962. Morphogenesis of the vertebrates. New York: Wiley.

Traquair, R.H. 1884. Description of a fossil shark (Ctenacanthus costellatus) from the Lower Carboniferous rocks of Eskdale, Dumfriesshire. Geological Magazine 3: 3-8.

Traquair, R.H. 1898. On Cladodus neilsoni (Traquair) from the Carboniferous Limestone of East Kilbride. Transactions of the Geological Society of Glasgow 11: 41-50.

Wang, N.Z. 1991. Two new Silurian galeaspids (jawless craniates) from Zhejiang Province, China, with a discussion of galeaspid-gnathostome relationships. In M.M. Chang, Y.H. Liu, and G.R. Zhang (editors), Early vertebrates and related problems of evolutionary biology: 4165. Beijing: Science Press.

Watson, D.M.S. 1937. The acanthodian fishes. Philosophical Transactions of the Royal Society of London (B) 228(549): 49-146.

Wijhe, J. W. van. 1922. Frühe Entwickelungsstudien des Kopf- und Rumpfskeletts von Acanthias vulgaris. Bijdragen tot der Dierkunde 22: 271-298.
Williams, M.E. 1985. The "cladodont level" sharks of the Pennsylvanian black shales of central North America. Palaeontographica, Stuttgart (A) 190: 83-158.

Williams, M.E. 1998. A new specimen of Tamiobatis vetustus (Chondrichthyes, Ctenacanthoidea) from the late Devonian Cleveland Shale of Ohio. Journal of Vertebrate Paleontology 18(2): 251-260.

Wolfram, K.E. 1984. The functional anatomy of the jaw suspension of Notorynchus cepedianus (Chondrichthyes, Selachii), with application to fossil forms. Unpublished M.Sc. thesis, University of Nebraska. 249 pp.

Woodward, A.S. 1889. Catalogue of the fossil fishes in the British Museum (Natural History). London: British Museum (Natural History).

Young, G.C. 1979. New information on the structure and relationships of Buchanosteus (Placodermi, Euarthrodira) from the early Devonian of New South Wales. Zoological Journal of the Linnean Society 66: 309-352.

Young, G.C. 1980. A new early Devonian placoderm from New South Wales, Australia, with a discussion of placoderm phylogeny. Palaeontographica, Stuttgart (A) 167: 10-76.

Young, G.C. 1986. The relationships of placoderm fishes. Zoological Journal of the Linnean Society 88: 1-57.

Young, J.Z. 1981. The life of vertebrates, 3rd. ed. Oxford: Oxford University Press.

Yu, X. 1998. A new porolepiform-like fish, Psarolepis romeri, gen. et sp. nov. from the Lower Devonian of Yunnan, China. Journal of Vertebrate Paleontology 18: 261-274.

Zangerl, R. 1973. Interrelationships of early chondrichthyans. In P.H. Greenwood, R.S. Miles, and C. Patterson (editors), Interrelationships of fishes: 1-14. London: Academic Press.

Zangerl, R. 1990. Two new stethacanthid sharks (Stethacanthidae, Symmoriida) from the Pennsylvanian of Indiana, U.S.A. Palaeontographica, Stuttgart (A) 213: 115-141.

Zangerl, R., and G.R. Case. 1976. Cobelodus aculeatus (Cope), an anacanthous shark from Pennsylvanian black shales of North America. Palaeontographica, Stuttgart (A) 154: 107-157.

Zangerl, R., and M. E. Williams. 1975. New evidence on the nature of the jaw suspension in Palaeozoic anacanthous sharks. Palaeontology 18(2): 333-341.

Zhu, M., X. Yu, and P. Ahlberg. 2001. A primitive sarcopterygian fish with an eyestalk. Nature 410: 81-84. 